\title{
Project W-551 Summary Information for Early LAW Interim Pretreatment System Selection
}

\author{
A. R. Tedeschi \\ CH2M HILL Hanford Group, Inc. \\ Richland, WA 99352 \\ U.S. Department of Energy Contract DE-AC27-99RL14047 \\ EDT/ECN: N/A UC: \\ Cost Center: Charge Code: \\ B\&R Code: $\quad$ Total Pages: 137
}

Key Words: Interim Pretreatment System, Project W-551, Early LAW, IPS, down-select, crossflow filtration, rotary microfiltration, fractional crystallization, caustic side solvent extraction, ion exchange

Abstract: This report provides summary data for use by the decision board to assess and select the final technology for project W-551, Interim Pretreatment System. This project will provide early pretreated low activity waste feed to the Waste Treatment Plant to allow Waste Treatment Plan Low Activity Waste facility operation prior to construction completion of the Pretreatment and High Level Waste facilities. The candidate solids separations technologies are rotary microfiltration and crossflow filtration, and the candidate cesium separation technologies are fractional crystallization, caustic-side solvent extraction, and ion-exchange using spherical resorcinol-formaldehyde resin. This document provides a summary of comparative data against prior weighted criteria to support technology selection. Supporting details and background for this summary are documented in the separate report, RPP-RPT-37741.

TRADEMARK DISCLAIMER. Reference herein to any specific commercial product, process, or service by trade name, trademark, manufacturer, or otherwise, does not necessarily constitute or imply its endorsement, recommendation, or favoring by the United States Government or any agency thereof or its contractors or subcontractors.

Printed in the United States of America. To obtain copies of this document, contact: Document Control Services, P.O. Box 950, Mailstop H6-08, Richland WA 99352, Phone (509) 372-2420; Fax (509) 376-4989.

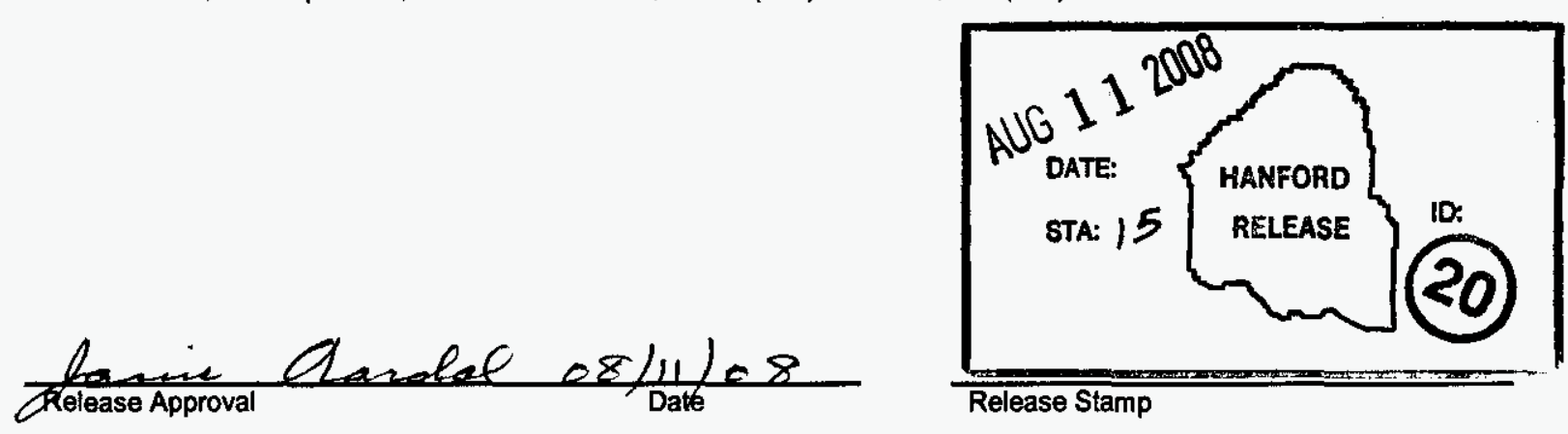

Approved For Public Release 


\begin{tabular}{|c|c|c|c|}
\hline \multicolumn{2}{|r|}{$\begin{array}{l}\text { Tank Farm Contractor (TFC) } \\
\text { RECORD OF REVISION }\end{array}$} & $\begin{array}{l}\text { (1) Document Number: } \\
\text { RPP-RPT-37740 }\end{array}$ & Page 1 \\
\hline \multicolumn{4}{|l|}{$\begin{array}{l}\text { (2) Title: } \\
\text { Project }\end{array}$} \\
\hline \multicolumn{4}{|c|}{ Change Control Record } \\
\hline \multirow{2}{*}{$\begin{array}{c}\text { (3) } \\
\text { Revision }\end{array}$} & \multirow{2}{*}{ (4) Description of Change - Replace, Add, and Delete Pages } & \multicolumn{2}{|c|}{ Authorized for Release } \\
\hline & & (5) Resp. Engr. (print/sign/date) & (6) Resp. Mgr. (print/sign/date) \\
\hline $0 \quad \mathrm{RS}$ & Initial Release & AR TedeschiOStast 8-7-08 & KA Colosi \\
\hline
\end{tabular}


RPP-RPT-37740

Revision 0

PROJECT W-551 SUMMARY INFORMATION FOR EARLY LAW INTERIM PRETREATMENT SYSTEM SELECTION

A. R. Tedeschi

CH2M HILL Hanford Group, Inc.
S. Schaus

K. Shah

C. Ervin

A E M Consulting, LLC

Date Published

August 2008

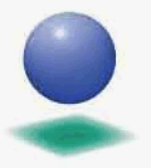

\section{CH2MHILL}

Hanford Group, Inc.

Post Office Box 1500

Richland, Washington

Prepared for the U. S. Department of Energy

Assistant Secretary for Environmental Management

Project Hanford Management Contractor for the

U.S. Department of Energy under Contract DE-AC06-96RL13200

Approved for public release; distribution is unlimited 
RPP-RPT-37740, Rev 0

This page intentionally left blank. 


\section{EXECUTIVE SUMMARY}

This report provides summary data for use by the Decision Board to assess and recommend the final technologies for project W-551, Interim Pretreatment System. This project will provide early pretreated low activity waste feed to the Waste Treatment Plant to allow Waste Treatment Plan Low Activity Waste facility operation prior to construction completion and startup of the Pretreatment and High Level Waste facilities. The candidate solids separations technologies are rotary microfiltration and crossflow filtration, and the candidate cesium separation technologies are fractional crystallization, caustic-side solvent extraction, and ion-exchange using spherical resorcinol-formaldehyde resin.

The basic data for this report was developed and assembled per the process defined in RPP-PLAN37558, "Decision Plan: Selection of Early LAW Interim Pretreatment System Processes for Removal of Entrained Solids and Cesium." This Decision Plan identified five main criteria-Safety, Regulatory/Stakeholder Acceptance, Technical Maturity/Flexibility, Operability and Maintainability, and Programmatic Aspects - to document performance of each candidate technology for the Interim Pretreatment System. In order to achieve a more objective and measurable assessment of the technologies by the Decision Board, these five criteria were subdivided into specific measures and definitions. Detailed assessment forms were produced for each technology to provide comparative data for the definitions. These assessment forms and other supporting details were documented in the separate document, RPP-RPT-37741, "Project W-551 Determination Data for Early LAW Interim Pretreatment Selection."

The data in each assessment form was then summarized and organized by technology. This report contains that organizational summary in both a short textual description and a cross-cutting matrix for each individual measure and definition. It also contains a summary of the basic design and operational information of the candidate technologies.

The information in this summary report was used as the initial data set by the Decision Board for technology assessment. Detailed data in RPP-RPT-37741 was used as necessary to clarify the summary descriptions. Assessment results are documented in the separate report RPP-RPT-38057, "Project $W$ 551 Interim Pretreatment System Technology Selection Summary Decision Report and

Recommendation." 
RPP-RPT-37740, Rev 0

This page intentionally left blank. 


\section{CONTENTS}

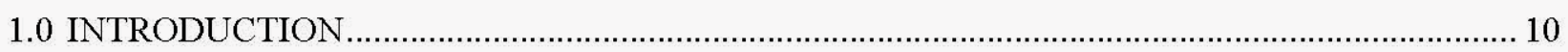

1.1 IDENTIFY VALUE FOR EARLY LAW OPERATION ............................................... 10

1.2 IDENTIFY CANDIDATE TECHNOLOGIES ............................................................ 11

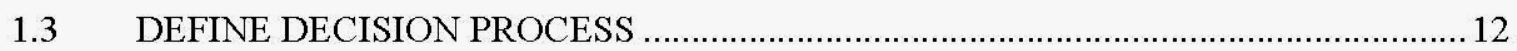

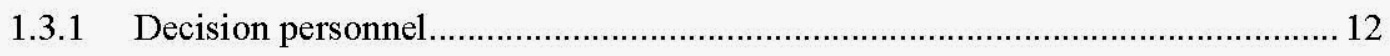

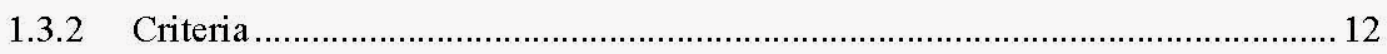

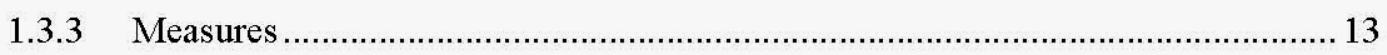

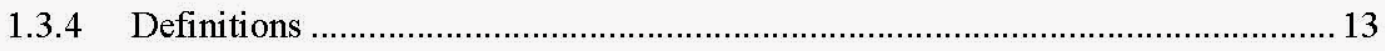

1.3.5 Assessment Forms .................................................................................. 13

1.4 DEVELOP COMPARATIVE EVALUATION DATA …....................................... 13

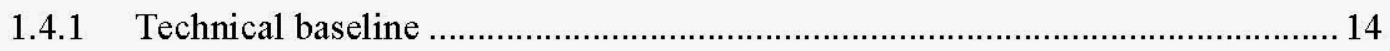

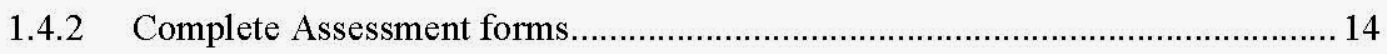

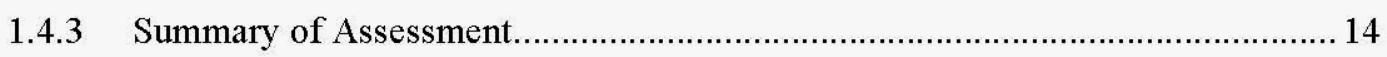

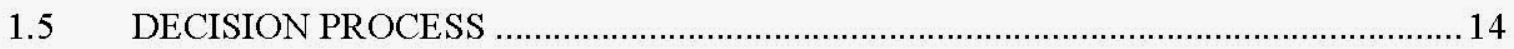

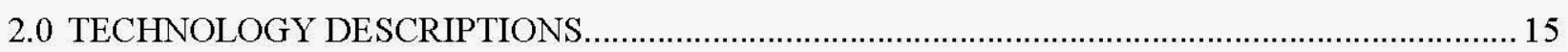

$2.1 \quad$ ENTRAINED SOLIDS SEPARATION TECHNOLOGIES …….............................. 15

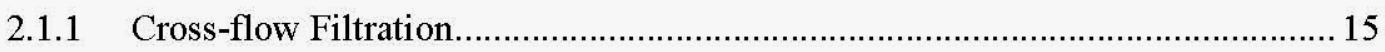

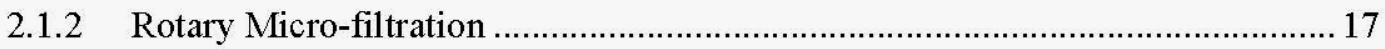

2.2 CESIUM SEPARATION TECHNOLOGIES .......................................................... 20

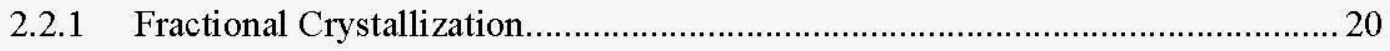

2.2.2 Caustic-Side Solvent Extraction ................................................................... 26

2.2.3 Ion Exchange using Spherical Resorcinol Formaldehyde Resin ........................ 32

3.0 ENTRAINED SOLIDS SEPARATION ASSESSMENTS ............................................................... 38

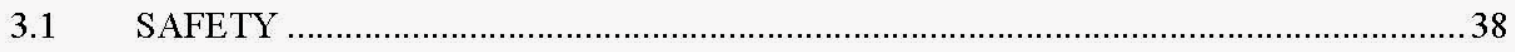

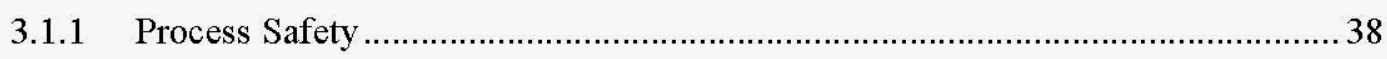

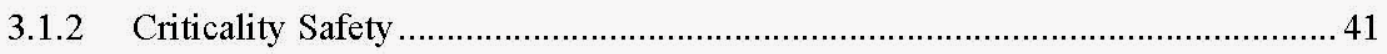

3.1.3 Industrial Safety and Hygiene.................................................................... 42

3.2 REGULATORY/STAKEHOLDER ACCEPTANCE …............................................. 43

3.2.1 Achieve Tribal Nations/Stakeholder Acceptance ............................................ 43

3.2.2 Achieve Regulator Acceptance............................................................... 43

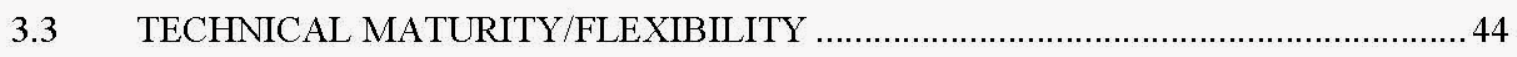

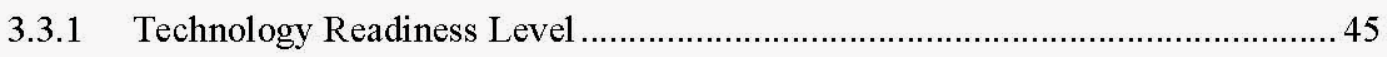

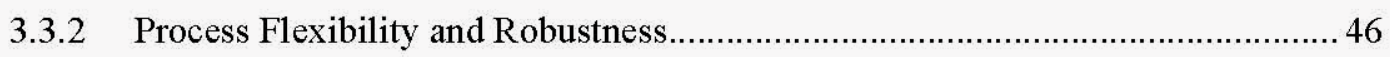

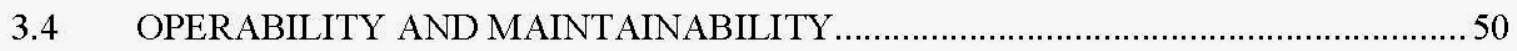

3.4.1 Ease of Process Control and Operation .................................................... 50

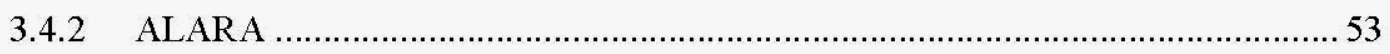

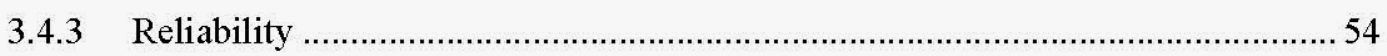

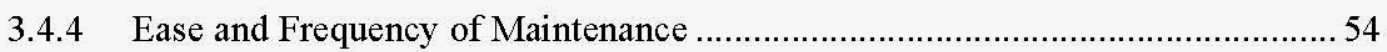




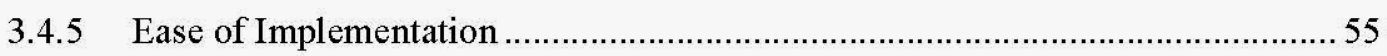

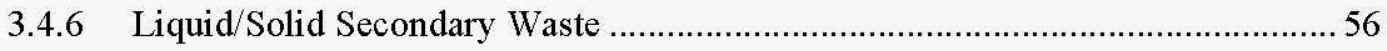

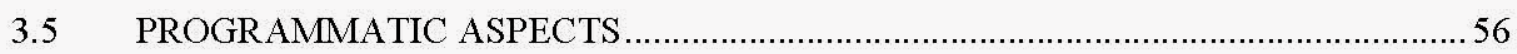

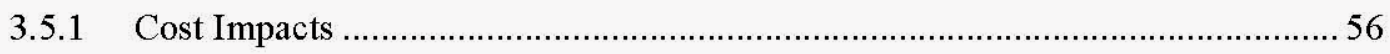

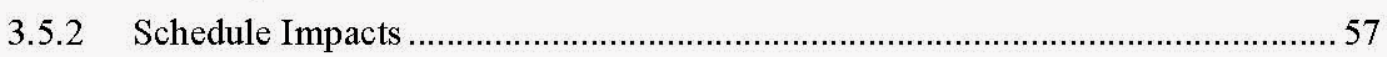

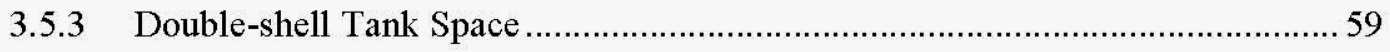

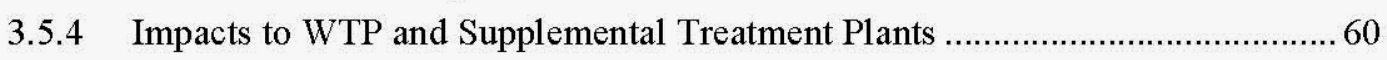

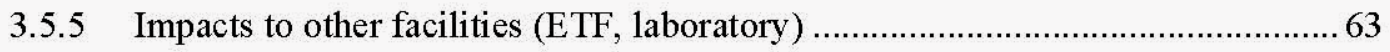

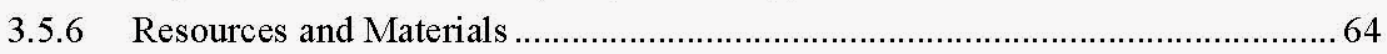

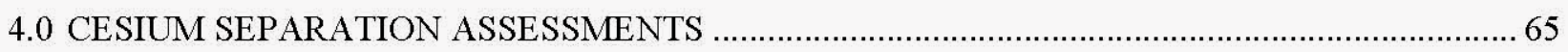

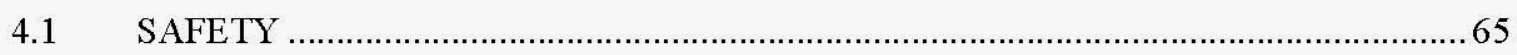

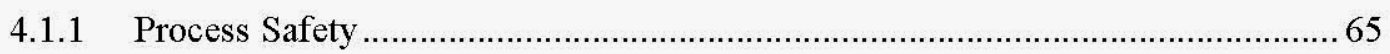

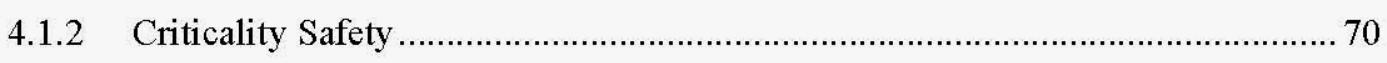

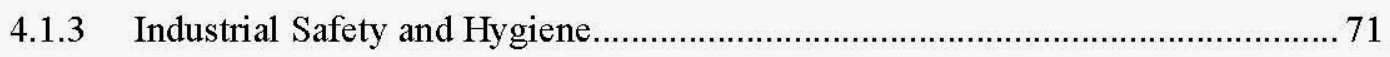

4.2 REGULATORY/STAKEHOLDER ACCEPTANCE …............................................... 71

4.2.1 Achieve Tribal Nations/Stakeholder Acceptance ............................................. 71

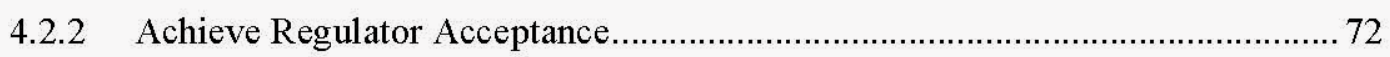

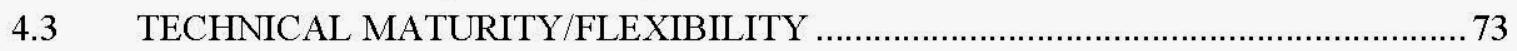

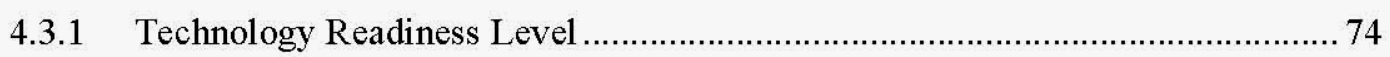

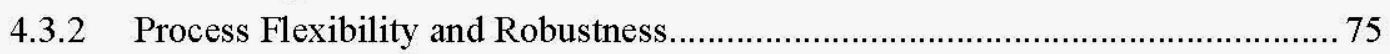

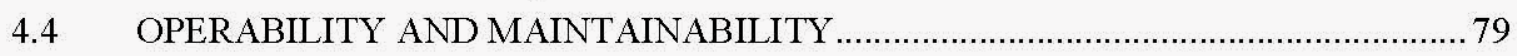

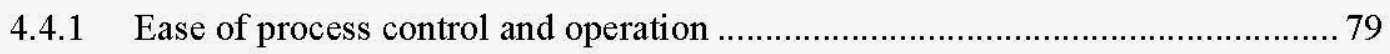

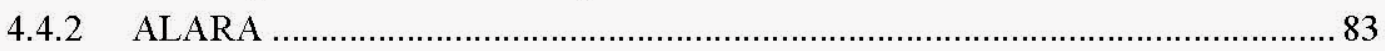

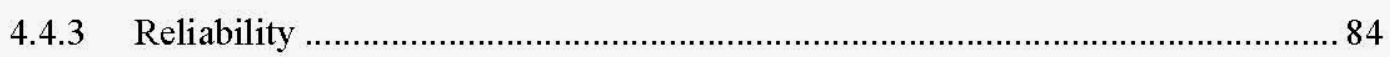

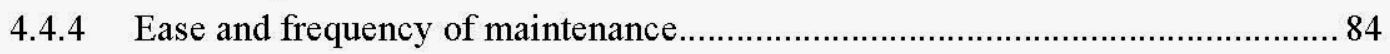

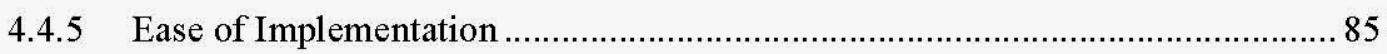

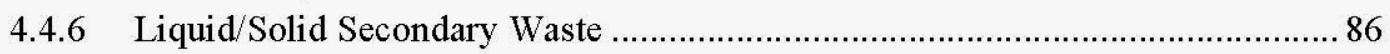

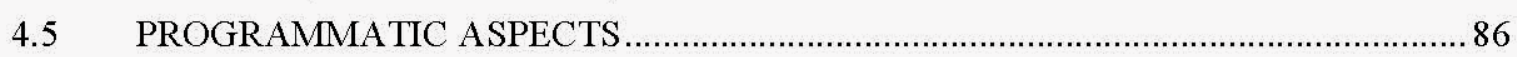

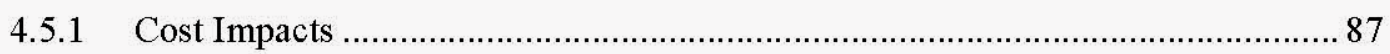

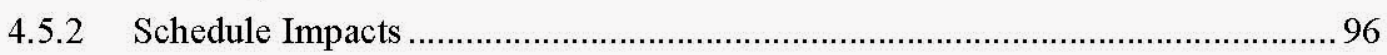

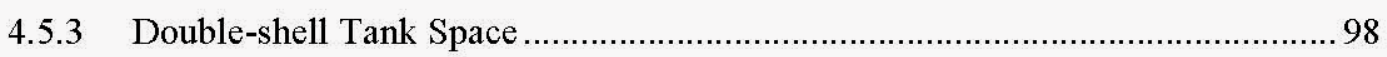

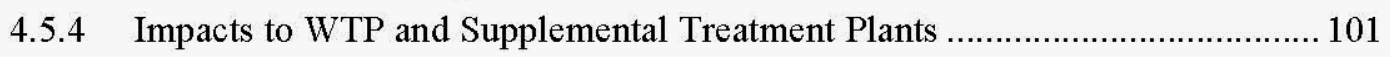

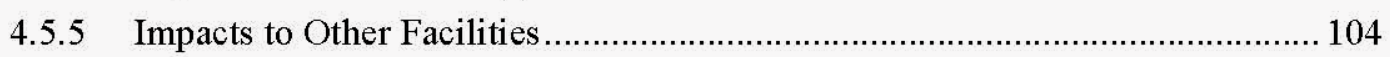

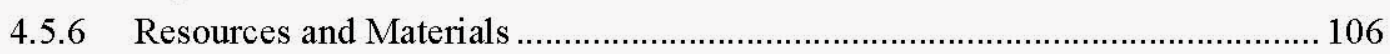

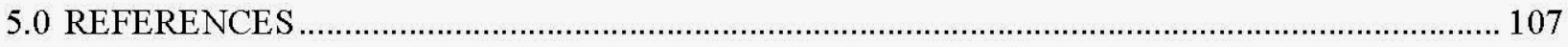




\section{LIST OF FIGURES}

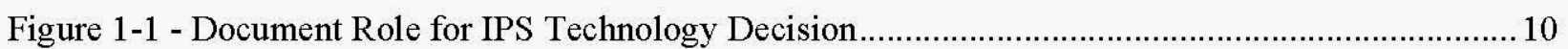

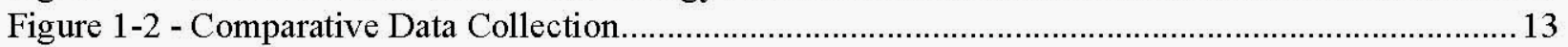

Figure 2-1 - Cross-flow Filtration Process Flow Diagram ............................................................... 18

Figure 2-2 - Conceptual Drawing of Rotary Micro-filter Module for In-tank Riser Installation ..............20

Figure 2-3 - Fractional Crystallization First Stage Process Flow Diagram ............................................24

Figure 2-4 - Fractional Crystallization Second Stage Process Flow Diagram ........................................25

Figure 2-5 - Caustic-Side Solvent Extraction Process Flow Diagram ....................................................... 30

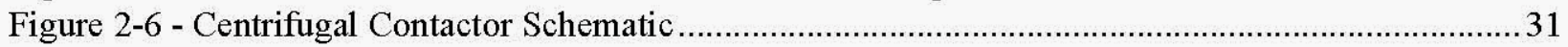

Figure 2-7 - Prototypical 2-Stage Contactor Unit ................................................................................. 31

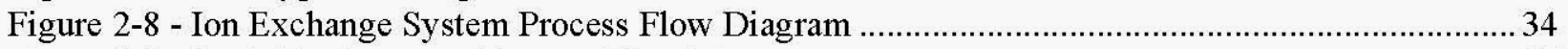

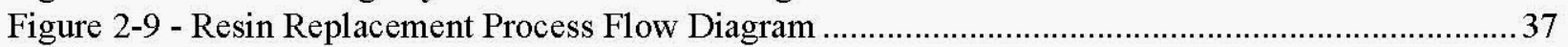

Figure 4-1 - Class 4 Capital Cost Estimate Summary for Project W-551 IPS Options ...........................88

Figure 4-2 - Class 4 Life-Cycle Cost Estimate Summary for Project W-551 IPS Options .......................89

Figure 4-3 - Project W-551 Life-Cycle Cost Profile for IX-sRF/CFF ..................................................90

Figure 4-4 - Project W-551 Life-Cycle Cost Profile for IX-sRF/RMF .................................................91

Figure 4-5 - Project W-551 Life-Cycle Cost Profile for FC/CFF .....................................................92

Figure 4-6 - Project W-551 Life-Cycle Cost Profile for FC/RMF..................................................... 93

Figure 4-7 - Project W-551 Life-Cycle Cost Profile for CSSX/CFF ....................................................99

Figure 4-8 - Project W-551 Life-Cycle Cost Profile for CSSX/RMF …….........................................95

Figure 4-9 - Net Change in Available Double-Shell Tank Waste Storage Space...................................99

Figure 4-10 - Net Change in Available Double-Shell Tank Waste Storage Space.................................. 100

Figure 4-11 - Net Change in Available Double-Shell Tank Waste Storage Space................................. 101

\section{LIST OF TABLES}

Table 3-1 - Potential Tank Farm Hazards from Filtration Technologies ................................................ 40

Table 3-2 -Total Pu-239 Estimation for IPS Feed Vector..................................................................... 42

Table 3-3 - Effort to Mature Solids Separation Technologies Comparison...............................................45

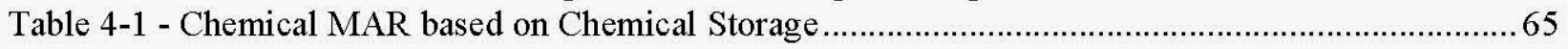

Table 4-2 - Potential Tank Farm Hazards from Cesium Separation Technologies .................................69

Table 4-3 - Effort to Mature Solids Separation Technologies Comparison...............................................75

Table 4-4 - Operation Components for Cesium Separation Processes .................................................. 81

Table 4-5 - Components and Equipment items for Maintenance ……................................................ 85

Table 4-6 - Durations of Major IPS Activities (not additive) …....................................................... 97

\section{ATTACHMENTS}
A DECISION CRITERIA, MEASURES, AND DEFINITIONS FOR INTERIM PRETREATMENT
SYSTEM TECHNOLOGIES
B SOLID SEPARATION TECHNOLOGY ASSESSMENT SUMMARY MATRIX
C CESIUM SEPARATION TECHNOLOGY ASSESSMENT SUMMARY MATRIX
D IPS SCHEDULES 


\section{LIST OF TERMS}

\begin{tabular}{|c|c|}
\hline AACE & Association for the Advancement of Cost Engineering \\
\hline ALARA & As low as reasonably achievable \\
\hline ANL & Argonne National Laboratory \\
\hline BNFL & British Nuclear Fuel Limited, Inc. \\
\hline CAA & Clean Air Act \\
\hline $\mathrm{CD}$ & Critical Decision \\
\hline $\mathrm{CFF}$ & Cross-flow filtration \\
\hline CH2M HILL & CH2M HILL Hanford Group, Inc. \\
\hline $\mathrm{COPC}$ & Constituents of potential concerns \\
\hline CSSX & Caustic side solvent extraction \\
\hline CWA & Clean Water Act \\
\hline $\mathrm{D}_{\mathrm{Cs}}$ & Cesium distribution coefficient \\
\hline DCS & Distributed control system \\
\hline $\mathrm{D} \& \mathrm{D}$ & Decontamination \& decommissioning \\
\hline DOE & U. S. Department of Energy \\
\hline DSA & Documented Safety Analysis \\
\hline DST & Double-shell tank \\
\hline EA & Environmental Assessment \\
\hline EIS & Environmental Impact Statement \\
\hline ESP & Environmental Simulation Program \\
\hline ETF & Effluent Treatment Facility \\
\hline FC & Fractional crystallization \\
\hline HEPA & High efficiency particulate air [filter] \\
\hline HLW & High level waste \\
\hline IDF & Integrated Disposal Facility \\
\hline IPS & Interim Pretreatment System \\
\hline ILAW & Immobilized low activity waste \\
\hline IX & Ion exchange \\
\hline LAW & Low-activity waste \\
\hline MAR & Materials at risk \\
\hline $\mathrm{MCU}$ & Modular Caustic-Side Solvent Extraction Unit \\
\hline MVST & Melton Valley Storage Tank \\
\hline NEPA & National Environmental Policy Act \\
\hline NESHAPS & National Emissions Standards for Hazardous Air Pollutants (CAA) \\
\hline NPDES & National Pollutant Discharge Elimination System (CWA) \\
\hline $\mathrm{NPH}$ & Natural Phenomenon Hazard \\
\hline $\mathrm{O \& M}$ & Operation \& Maintenance \\
\hline ORNL & Oak Ridge National Laboratory \\
\hline ORP & Office of River Protection \\
\hline PPE & Personnel protection equipment \\
\hline RCRA & Resource Conservation and Recovery Act \\
\hline $\mathrm{RMF}$ & Rotary micro-filtration \\
\hline ROD & Record of decision \\
\hline ROM & Rough order-of-magnitude \\
\hline RPP & River Protection Project \\
\hline SEPA & State Environmental Policy Act \\
\hline SME & Subject matter expert \\
\hline sRF & Spherical resorcinol-formaldehyde \\
\hline
\end{tabular}




\section{LIST OF TERMS continued}

SRNL

SRS

SST

TC\&WM

TEEL

$\mathrm{TF}$

TOA

TOE

TRL

TSD

ULD

WAC

WAC

WIR

WTP

WVDP
Savannah River National Laboratory

Savannah River Site

Single-shell tank

Tank Closure \& Waste Management

Temporary Emergency Exposure Limits

Tank Farms

Tri-octyl-amine

Total Operating Efficiency

Technology Readiness Level

Treatment, storage, and disposal

Unit Liter Dose

Waste Acceptance Criteria

Washington Administrative Code

Waste Incidental to Reprocessing

Waste Treatment Plant

West Valley Demonstration Project 


\subsection{INTRODUCTION}

Project W-551 will construct and operate the Interim Pretreatment System (IPS) to supply low activity waste (LAW) feed to the Waste Treatment Plant (WTP). Construction of the WTP LAW treatment system is expected to be completed at least five years prior to the WTP pretreatment facility. The IPS will thus allow earlier operation of the WTP LAW treatment facility by providing an early feed supply.

CH2M HILL Hanford Group, Inc. (CH2M HILL) was chartered by the Department of Energy, Office of River Protection (ORP) to assess several viable solids separation and cesium separation technologies for the IPS, and begin further project pre-conceptual effort on the selected technologies. The candidate technologies for entrained solids removal were cross-flow filtration and rotary micro-filtration. The candidate technologies for cesium separations were fractional crystallization, caustic-side solvent extraction, and ion exchange using spherical resorcinol-formaldehyde resin.

The process used by CH2M HILL to provide a recommendation to ORP is shown below in Figure 1-1. This document and its companion report are highlighted in this figure. Information in this section is organized by these process steps.

Figure 1-1 - Document Role for IPS Technology Decision

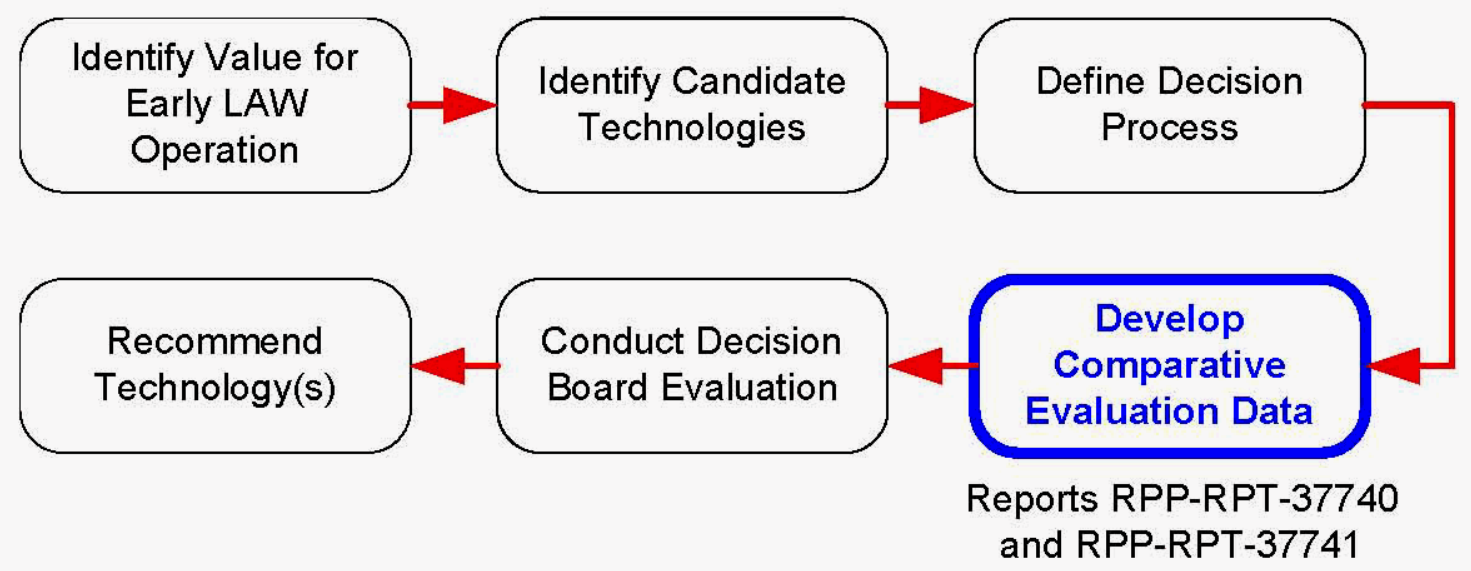

\subsection{IDENTIFY VALUE FOR EARLY LAW OPERATION}

Construction of the WTP LAW Vitrification facility is anticipated to be completed by 2014, whereas the WTP Pretreatment facility is scheduled for completion by 2019. There is insufficient Hanford tank waste as it exists in the tanks to run the WTP LAW facility during this five year interim period; the WTP LAW facility requires the WTP Pretreatment facility to provide it conditioned Hanford tank waste feed for its vitrification process. This schedule disconnect, caused by changes in design application, will result in the WPT LAW facility remaining inactive for at least five years after its construction completion.

- Starting up the WTP LAW facility without this delay has the following advantages:

- Supports early Hanford tank closure by early treatment of tank waste, thus minimizing environmental risk and mitigating future operational costs 
- Maintaining efficiency in commissioning and startup of the WTP LAW facility by ensuring staff and knowledge continuity

- Provides risk minimization of future schedule delays in WTP Pretreatment

- Supports accelerated single-shell tank retrieval.

This short summary value list is amplified in the two evaluation reports, RPP-29981, Evaluation of Starting the Waste Treatment and Immobilization Plant (WTP) Low Activity Waste (LAW) Facility First (CH2M HILL 2007), and RPP-RPT-30160, Supporting Information for the Evaluation of Starting the Waste Treatment and Immobilization Plant (WTP) Low Activity Waste (LAW) Startup First Scenarios (CH2M HILL 2006).

Based upon the value of starting up the WTP LAW facility, ORP commissioned CH2M HILL to begin work on constructing the necessary precursor facility: a reduced-function interim pretreatment facility, to primarily provide conditioned waste feed for WTP LAW five year operation. The two functions of this IPS during this five year period will be to remove entrained solids and reduce radioactive cesium concentration of select Hanford tank wastes. Project W-551, "Interim Pretreatment System," was defined to implement this direction.

The requirement to pretreat tank wastes is derived from a waste incidental to reprocessing (WIR) determination prepared by the U.S. Nuclear Regulatory Commission. Removal of entrained solids and ${ }^{137} \mathrm{Cs}$ from tank wastes (e.g., supernatant and salt cake) is required to meet the WIR requirements for these wastes. Once the entrained solids and ${ }^{137} \mathrm{Cs}$ are removed to acceptable levels, supernatant and salt cake wastes are considered to be the LAW and can be immobilized in glass and disposed at the Hanford Site Integrated Disposal Facility (IDF). The WIR determination established a technical and economical processing limit of $5.0 \mathrm{E}-02 \mathrm{Ci} / \mathrm{L}$ (normalized to $7 \underline{\mathrm{M}}$ sodium) for the ${ }^{137} \mathrm{Cs}$ concentration in LAW. Wastes with a ${ }^{137} \mathrm{Cs}$ concentration less than $5 \mathrm{E}-02 \underline{\mathrm{Ci}} / \mathrm{L}$ (normalized to $7 \underline{\mathrm{M}}$ sodium) do not require cesium removal in order to meet WIR criteria. While the ${ }^{137} \mathrm{Cs}$ concentration in some single-shell tank salt cake waste is lower than this limit, the ${ }^{137} \mathrm{Cs}$ concentration in the double-shell tank (DST) supernatant exceeds this limit. Based on its design and operating concept, further reduction of the ${ }^{137} \mathrm{Cs}$ concentration in LAW processed by the WTP LAW Vitrification facility is required in order to maintain personnel radiation exposure As Low As Reasonably Achievable (ALARA). The maximum ${ }^{137} \mathrm{Cs}$ concentration in waste processed by the IPS is required to be $1.68 \mathrm{E}-05 \mathrm{Ci} / \mathrm{g}-\mathrm{mol} \mathrm{Na}(\sim 5 \mathrm{E}-04 \mathrm{Ci} / \mathrm{L})$.

\subsection{IDENTIFY CANDIDATE TECHNOLOGIES}

Several technologies have been previously demonstrated by the U.S. Department of Energy (DOE) for separating entrained solids from solutions. These solid separation technologies include:

- Gravity settling

- Centrifugation

- Mechanical filtration

Cross-flow filtration (CFF)

Rotary micro-filtration (RMF)

Based on previous work, it was determined by ORP that mechanical filtration systems for entrained solids removal are needed for the IPS (ORP 2008).

The DOE has also investigated several cesium removal technologies over the past 50 years. These cesium removal technologies include: 
- Selective dissolution

- Precipitation

- Solvent extraction

- Ion exchange, using

Elutable resin

Non-elutable resin

For DST supernatant pretreatment in the IPS, three cesium separation technologies were identified by ORP for further assessments (ORP 2008). These three technologies are:

- Fractional Crystallization (FC)

- Caustic side solvent extraction (CSSX), and

- Ion exchange using spherical resorcinol-formaldehyde resin (IX-sRF)

The IPS is not intended to replace the WTP Pretreatment Facility, since the IPS will not have the full functionality or capacity of the WTP Pretreatment Facility. Using a solids and cesium separation technology on select Hanford tank wastes, in a less complex facility than the WTP Pretreatment Facility, will allow WTP LAW vitrification during the five year interim schedule period.

\subsection{DEFINE DECISION PROCESS}

The RPP-PLAN-37558, Decision Plan: Selection of Early LAW Interim Pretreatment System Process for Removal of Entrained Solids and Cesium (CH2M HILL 2008a), documents the process of selecting one entrained solids filtration and one cesium separation technology for the IPS. The following major steps summarize this process from this Decision Plan.

- Identify decision personnel, key stakeholders, and subject matter expert roles and responsibilities

- Define main subject areas for technology comparative analysis

- Qualify these subject areas into discrete measures and definitions

- Provide the framework for data collection and presentation to address the measures and definitions

- Establish initial weighting of the measures and definitions for usage by a Decision Board

- Identify follow-on actions, such as an independent Expert Review Panel review, and optional criteria planning in the event that initial mathematical weighting is not conclusive

\subsubsection{Decision personnel}

The decision-making team includes the ORP Decision Maker/Federal Project Director; a Decision Board comprised of contractor staff; subject matter experts (SMEs); and an Expert Review Panel. The Decision Board, convened by the Decision Maker, provides technical and management support to the Decision Maker. The SMEs provide information and support to the Decision Board to clarify the technology performance documented in the detailed assessments.

\subsubsection{Criteria}

A workshop was conducted on April 10, 2008 to jointly define the main subject areas for technology comparative analysis. This workshop involved decision personnel, stakeholders, and ORP. The results $\mathrm{r}-$ established the following main subject area criteria for comparative analysis: 
- Safety

- Regulatory/Stakeholder Acceptance

- Technical Maturity/Flexibility

- Operability and Maintainability

- Programmatic Aspects.

Each subject area was then assigned a percentage value "weighting factor."

\subsubsection{Measures}

The subject area criteria were further divided into various important "measures." Measures were developed to show how well each of the five criteria could be achieved. A total of nineteen (19) measures were developed covering these five criteria. In addition to each criterion, each measure was also assigned a "ranking factor" commensurate with its importance. Within a given criterion, the sum of the ranking factors equaled $100 \%$.

\subsubsection{Definitions}

To improve discriminators and identify differences among these technologies, each measure was then subdivided into specific measurable conditions called definitions. Each definition represented specific items for which qualitative or quantitative comparisons can be made. Attachment A provides a complete list of all criteria, measures and definitions identified, along with assigned weighting and ranking factors from the Decision Plan (CH2M HILL 2008a).

\subsubsection{Assessment Forms}

An Assessment Summary Form was developed to document the data required for each definition. The Assessment Summary Form includes the Assessment Scope, Conditions, Other Considerations, References, and an Assessment Summary. The described impacts can be positive as well as negative.

\subsection{DEVELOP COMPARATIVE EVALUATION DATA}

Collection of data for comparative analysis is depicted below in Figure 1-2.

Figure 1-2 - Comparative Data Collection

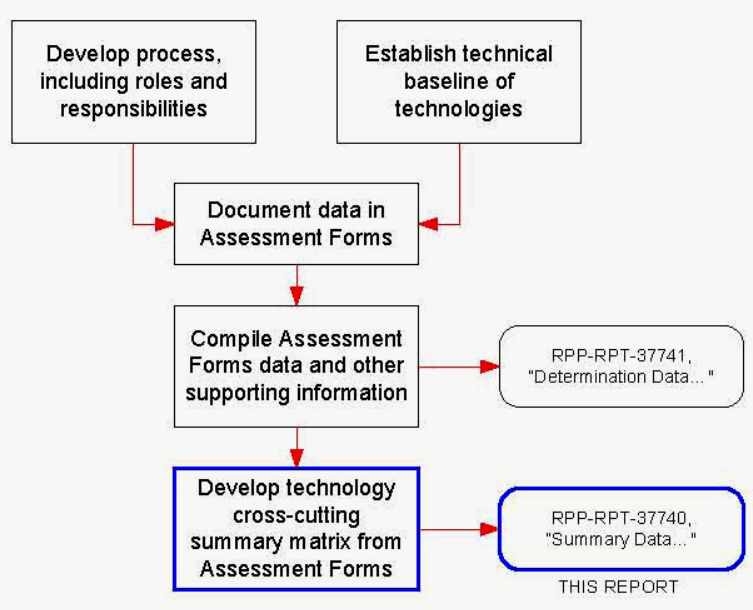




\subsubsection{Technical baseline}

The first step in developing comparative data was to establish a technical baseline. This baseline for each technology included process flow diagrams, mass balances and other technical information, and was documented in RPP-RPT-37551, Project W-551 Interim Pretreatment System Pre-Conceptual Candidate Technology Descriptions (CH2M HILL 2008c). Summaries for each technology from this report are included in Section 2. This data was the beginning basis for providing an assessment of each technology against the Decision Plan criteria, measures, and definitions, by establishing a referenceable system design and application.

\subsubsection{Complete Assessment forms}

Subject matter experts then gathered data from test and report documents, design information, and working group evaluations to assemble detailed assessments of each technology per definition line. Compilation of the Assessment Forms and other supporting information are documented in the report, RPP-RPT-37741, Project W-551 Determination Data for Early LAW Interim Pretreatment Selection (CH2M HILL 2008d).

\subsubsection{Summary of Assessment}

This report documents a summary of the data included in RPP-RPT-37741 (CH2M HILL 2008d). It was decided to issue two separate documents, one of the detailed data and the other a summary, to provide a simpler reference for the Decision Board and follow-up reviewers. (The RPP-RPT-37741 is large document of over 800 pages.)

Summaries of the qualitative assessments for entrained solids filtration and cesium separation are provided in Sections 3 and 4, respectively. These summaries were used in the review process performed by the Decision Board and the ORP Decision Maker. Summary comparisons from the assessments were then documented into a table, to assist the Decision Board. This table was organized by technology to allow a cross-cutting matrix comparison. The table for solids separation technology is included as Attachment B and the cesium separation technology table is included as Attachment C.

Summary assessments provided herein are both qualitative and quantitative in nature. While the summaries provide a ranking if possible, the final evaluation of the weighting and discriminator importance are provided by the Decision Board.

\subsection{DECISION PROCESS}

The summary data contained herein and the supporting details documented in RPP-RPT-37741 (CH2M HILL 2008d) were used by the Decision Board to compare the technologies, and establish a numerical ranking for quantitative evaluation. This process and results are documented in the report, RPP-RPT38057, Project W-551 Interim Pretreatment System Technology Selection Summary Decision Report and Recommendation (CH2M HILL 2008e). 


\subsection{TECHNOLOGY DESCRIPTIONS}

Summarized descriptions of the five technologies being evaluated are provided in this section. Detailed descriptions and processing characteristics of these technologies are documented in RPP-RPT-37551, Interim Pretreatment System Pre-Conceptual Candidate Technology Descriptions (CH2M HILL 2008c).

\subsection{ENTRAINED SOLIDS SEPARATION TECHNOLOGIES}

The waste stored in the DSTs contains a small quantity (typically less than $0.5 \mathrm{wt} \%$ ) of insoluble solids that are suspended in the supernatant. These entrained solids may contain ${ }^{90} \mathrm{Sr}$ and transuranic (TRU) elements that if not removed could adversely affect the cesium separation process and result in the immobilized LAW (glass) exceeding limits for these radionuclides. As stated earlier, several technologies have been previously demonstrated by the DOE for separating entrained solids from solutions. Based on previous testing, both $\mathrm{CFF}$ and $\mathrm{RMF}$ are capable of meeting the solids removal requirements. These two technologies are summarized below.

\subsubsection{Cross-flow Filtration}

Cross-flow filtration uses a tube bundle to capture solids while filtrate passes through the filter tube. In cross-flow filtration, the feed is passed across the filter membrane (tangentially to the filter membrane) at some pressure difference. Material which is smaller than the membrane pore size passes through the membrane as permeate or filtrate, and everything else is retained on the feed side of the membrane as retentate. This mode of operation reduces blinding, where the accumulated solids hinder liquid flow; liquid supply flow is tangential the filtrate flow through the membrane thus providing a shear/scouring effect.

\subsubsection{Technology Development Summary}

Cross-flow filtration has been used successfully in radioactive service at multiple DOE sites including West Valley Demonstration Project (WVDP), the Savannah River Site (SRS) and Oak Ridge National Laboratory (ORNL). The cross-flow filter unit used at ORNL was part of the Wastewater Triad Project, which included a cesium removal system (ion exchange columns) and an out-of-tank evaporator system. Cross-flow filtration has been selected for solid-liquid separation at the WTP Pretreatment facility after evaluating candidate technologies.

The cross-flow filter consists of two Mott HyPulse ${ }^{\text {TM }}$ LSX Filter modules connected in series, each consisting of a $5 \mathrm{ft}$-long-bundle of 31 elements with a 0.75 -in. outside diameter and a $0.5 \mu \mathrm{m}$ pore size. At ORNL, the Melton Valley Storage Tank (MVST) waste feed to the cross-flow filter unit contained up to $22 \mathrm{wt} \%$ solids. The filtrate flux rate for the ORNL cross-flow filter was between 0.012 and $0.16 \mathrm{gpm} / \mathrm{ft}^{2}$. The alpha concentration (primarily associated with the solids) in the MVST feed to the cross-flow filter was reduced by $>99.9 \%$ and solids content of the filtrate was $\sim 0.02 \mathrm{wt} \%$ during initial testing in 1999 .

ORNL hot operations in 1999 processed a total volume of waste during two campaigns of about 45,000 gal. Filtrate flux rates were dependant on solids concentration with reduced flux rates at higher undissolved solids concentrations. Filter performance correlated well with design filtrate production rates. The quality of the filtrate consistently met the requirements for feed to the downstream ion exchange and evaporation processes. The modular system, including pumps, valves, instrumentation, shielding, and containment, experienced a high degree of reliability and operability. 
Pilot-scale testing using simulants of AN-105 (Envelope A) and AN-107 (Envelope C) wastes was performed early in the WTP design phase by BNFL (BNF-003-98-0221 and BNF-003-98-0226). Flux rates ranged between 0.10 and $0.16 \mathrm{gpm} / \mathrm{ft}^{2}$ at solids concentrations of 0.5 to $16 \mathrm{wt} \%$. These tests demonstrated the flux rate dependence on axial velocity and recommended a velocity of $12 \mathrm{ft} / \mathrm{sec}$ with a transmembrane pressure of 40 to $55 \mathrm{psi}$ (differential) for best filter performance. The effectiveness of frequent back-pulse for maintaining flux rates was also demonstrated.

Excluding Sr/TRU precipitation and filtration tests, only two cross-flow filtration tests have been conducted with actual tank waste from AW-101 and AN-104 (WSRC-TR-2002-00530 and WSRC-TR2003-00295). These tests produced average filter fluxes of between 0.050 and $0.085 \mathrm{gpm} / \mathrm{ft} 2$ at low solids concentrations $(<1 \mathrm{wt} \%$ ), with axial velocities of approximately $11 \mathrm{ft} / \mathrm{sec}$ and transmembrane pressures from $40 \mathrm{psi}$ to $60 \mathrm{psi}$.

\subsubsection{Process Description}

The proposed cross-flow filter process uses multiple $0.1-\mu \mathrm{m}$ sintered-metal tubes enclosed within a shell. Waste flows axially through the tube (parallel to the filter media) and filtrate passes radially through the tube wall driven by a differential pressure between the inside of the tubes and the shell. High flow velocity through the tubes produces a shear at the inside tube wall that reduces the buildup of a particulate layer. Some build up of solids is expected; however, so periodic back-pulse of the filter and chemical cleaning is required to remove these solids and to maintain the filter flux rate.

The pore size of $0.1-\mu \mathrm{m}$ is chosen because it has demonstrated higher flux rates than the $0.5-\mu \mathrm{m}$ filter in cross-flow filter testing. This is likely a result of the smaller particle sizes in Hanford waste and simulant, which tend to more readily clog the pores of the $0.5-\mu \mathrm{m}$ filter.

A high-capacity pump circulates waste supernate from the filter feed vessel through the cross-flow filter unit. The circulating pump is a "low-shear" type to avoid reducing the particle size distribution of the suspended solids. Suspended solids with small particle size tend to plug the pores of the cross-flow filters, resulting in a decrease in filtrate production. The filtrate passes through the filter elements to the shell side of the tube bundles and is delivered to the cesium separation process. The concentrate passes through a regulating valve (used to control differential pressure across the filter elements) and is collected in the cross-flow filter feed vessel. Fresh waste supernate is added to the feed vessel to replenish filtrate removed from the system. A pressurized back-pulse vessel is used to periodically clean the cross-flow filter elements with filtrate to minimize solids accumulation and fouling of filter membrane. Chemical cleaning of the filter with sodium hydroxide may also be used to remove deep fouling from the filter tubes. The cleaning fluids are collected in the filter feed vessel.

The cross-flow filter unit is housed in a shielded module adjacent to an existing DST and collocated with the cesium removal process. LAW feed solution contained within the DST is transferred through a shielded above ground transfer pipeline to the cross-flow filter unit. The clarified LAW supernatant is transferred to the cesium separation process while the concentrate is returned to a DST adjacent to the feed tank.

Filtrate quality and removal efficiency has not been quantitatively determined. In some testing, the solids content in the filtrate was below the resolution of the measurement instrument. The solids removal efficiency is assumed to be $99.99 \%$ in the material balance, which is consistent with the basis used for the WTP.

Testing has been conducted on both actual and simulated Hanford waste to determine cross-flow filter flux rates. However, it has been shown that bench-scale performance data typically overestimate the 
filtrate flux rate at larger scale, so test data cannot be directly applied to design (WSRC-MS-2006-00115). The WTP flow sheet contains a variable flux rate depending on wt $\%$ solids and sodium molarity that is based on an evaluation of test data. The cross-flow filters are assumed to remove $99.99 \%$ of solids and the feed stream is concentrated to $20 \mathrm{wt} \%$ solids (24590-WTP-RPT-PT-02-005).

\subsubsection{Process Flow diagram}

Figure 2-1 shows the process flow diagram for the CFF system.

\subsubsection{Rotary Micro-filtration}

Rotary micro-filtration employs a series of rotating filters disks where filtrate is passed through the sandwiched filter membrane using pressure drop and tangential rotation force.

\subsubsection{Technology Development Summary}

Savannah River National Laboratory (SRNL) received funding from DOE EM-21, Office of Cleanup Technologies, to develop the rotary micro-filter for high-level radioactive service. The work focused on evaluating alternative rotary micro-filter vendors, redesigning the equipment for radioactive service, engineering studies to evaluate the risks, determining downstream impacts, assessing costs and benefits of deploying this technology, performing actual waste and pilot-scale testing of the technology, and evaluating alternative filter media. This work has culminated in the decision to design, fabricate and perform testing on a full-scale rotary micro-filter for potential SRS Tank Farm applications (WSRC-STI2008-00050).

In 2001 initial bench-scale testing began with an off-the-shelf, single disk, rotary micro-filter, which led to additional pilot-scale testing in the following years. Both actual waste and simulant testing was performed as well as materials irradiation testing and evaluations. This testing showed good performance with generally higher flux rates than cross-flow filtration. Bench-scale testing demonstrated flux rates of 2 to 10 times that of cross-flow filtration and pilot-scale testing demonstrated flux rates of 1.5 to 2.8 times. The SRS has developed and tested a full-scale radiation hardened SpinTek ${ }^{\mathbf{T M}}$ rotary micro-filter unit (WSRC-STI-2006-00073) and has designed a system to be deployed in the SRS underground storage tank. Testing of the unit demonstrated continued ability to meet filtrate quality objectives and suggested further design improvements. Full-scale SpinTek ${ }^{\text {TM }}$ used for testing by SRS is undergoing testing using Hanford waste simulant.

The SRS unit consists of two filter modules designed to fit within a 48-inch diameter riser on the tank. The SpinTek ${ }^{\mathrm{TM}}$ rotary micro-filter would need to be re-designed to fit within a 42 -inch diameter riser on a Hanford DST and the pump suction legs would need to be extended. These modifications appear possible.

The SRS design modifies the standard SpinTek ${ }^{\text {TM }}$ ST-II, 25-disk model for use in a radioactive environment. Modifications include use of more radiation-tolerant materials; use of a modular design that contains the filter stack, all seals and rotary unions within a removable unit; and seal and bushing modifications to mitigate areas of high-wear experienced during testing.

\subsubsection{Process Description}

The rotary micro-filter unit uses sintered metal disks available in $0.1-\mu \mathrm{m}$ or larger pore sizes. The pore size of $0.1-\mu \mathrm{m}$ is chosen because it has demonstrated higher flux rates than the $0.5-\mu \mathrm{m}$ filter in cross-flow filter testing. This is likely due to the smaller particle sizes in Hanford waste and simulant, which tend to more readily clog the pores of the $0.5-\mu \mathrm{m}$ filter. 
Figure 2-1 - Cross-flow Filtration Process Flow Diagram

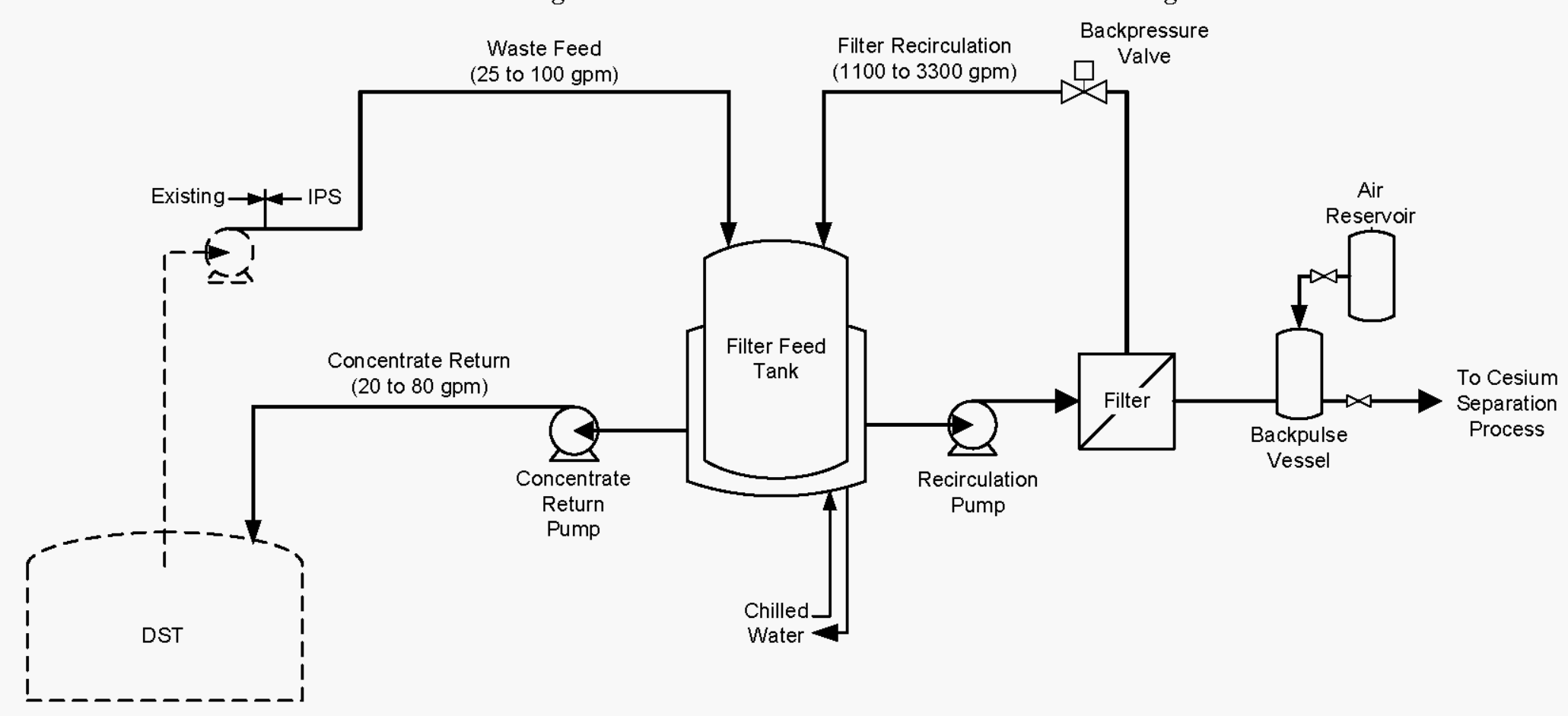


The disks are hollow with the sub-micron membrane on each disk surface. The disks are spaced along a hollow central shaft that spins inside a pressurized housing and the differential pressure between the housing and the inside of the disks drives filtrate across the membrane. An external motor rotates the central shaft and disk assembly. The speed of the disk rotation can be adjusted to increase the shear forces at the surface of the disks. The shear force disrupts particulate deposition mechanisms and aids in minimizing the thickness of the particulate layer that builds up on the membrane, thus enhancing the filtrate flux rate. The efficiency of this fluid shear, or "sweeping action," increases with the velocity of the fluid. Stationary spoke turbulence promoters are positioned above and below each disk, which also increases the shear rate at the surface of the membrane by minimizing the boundary layer.

The feed slurry is pumped into the filter housing and flows across the external surface of the rotating filter disks. A transmembrane pressure gradient drives the supernate through the filter membrane and into the center of the hollow disks. A valve on the concentrate exit automatically controls the pressure inside the filter housing. This provides the transmembrane pressure required to force filtrate through the filter membranes. The filtrate moves to the center of the disk and collects in the shaft holding the disks. The filtrate is discharged from the central shaft to the cesium removal process. Concentrated feed slurry exits the filter housing to be returned to the DST.

The rotary micro-filter unit with feed pumps is housed in a module that is inserted through an existing riser on an existing DST (see Figure 2-2). The LAW feed solution contained within the DST is transferred through the rotary micro-filter unit. The clarified LAW supernatant is transferred to the cesium separation process while the solid concentrate is discharged back into the DST. Periodic cleaning of the rotary micro-filter elements is conducted with water, sodium hydroxide, or nitric acid to minimize solids accumulation and fouling of filter membrane. 
Figure 2-2 - Conceptual Drawing of Rotary Micro-filter Module for In-tank Riser Installation ${ }^{1}$

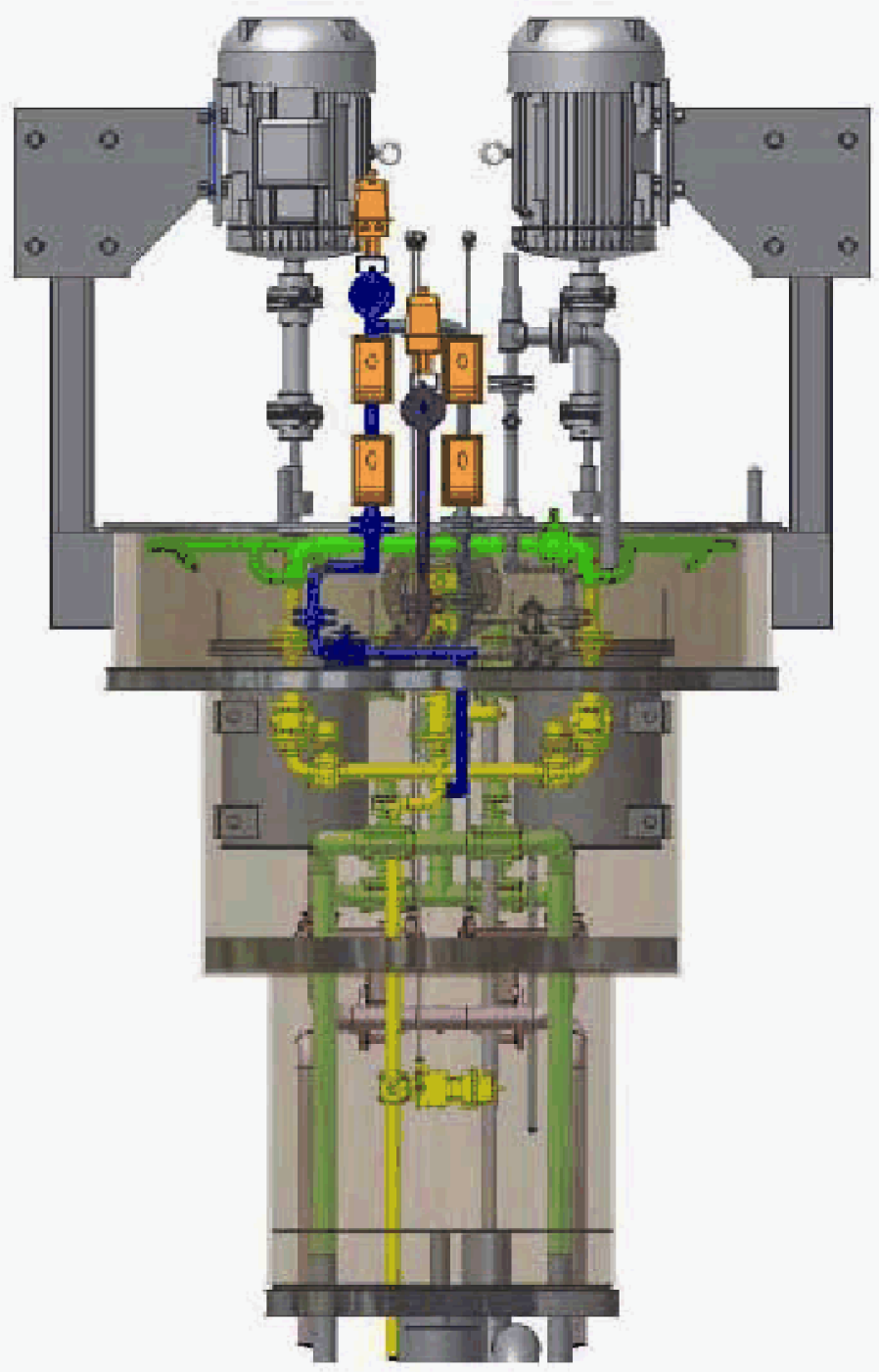

\subsection{CESIUM SEPARATION TECHNOLOGIES}

Three catididate technologies are evaluated for cesim separation: fractional ctystallization, solvent extraction, and ion exchange. The following discussion prowides detals of the proposed deployment in the IPS

\subsubsection{Fractional Crystallization}

In the Fractional Crystallization (FC) process, waste is concentrated by ewat oration until sodium salts exceed their solubility limits. Cesium and other soluble isotopes temain in the liquid phase (liquor) while sodium salts form solid ctystals. The li quor is separated from the corystals which represent the decontaminated product. Decontaminated sodium salt crystals may be produced as a solid product or may be completely or patially te-dissolved with water to produce a li quid or slury product.

\footnotetext{
${ }^{1}$ Ex cerft.from WSRC-STI-2008-00050.
} 


\subsubsection{Technology Development Summary}

Since December 2004 a program has been actively pursued to develop fractional crystallization for treatment of Hanford tank waste. This program has progressed from laboratory testing with simulants and actual waste through engineering scale tests of a 20 -liter continuous crystallizer system and solids liquid separation equipment. A pilot plant with approximate 5000-liter crystallizer has been constructed and is currently undergoing startup tests.

Similar to industrial processes, crystallization of Hanford wastes has been practiced in the Hanford 242-A and 242-S evaporators for many years. The operational 242-A evaporator is used to reduce waste volume by evaporating water and crystallizing sodium salts from Hanford waste. However, the crystallized salts are not separated and decontaminated from the residual liquor at the $242-\mathrm{A}$ evaporator. Some incidental fractional crystallization separation is performed in the tank farms. As the evaporator product cools and ages in the underground tanks, lower solubility salts crystallize and settle. By removal of supernate and interstitial liquor, content of ${ }^{137} \mathrm{Cs},{ }^{99} \mathrm{Tc}$, and other high solubility components are reduced in the resulting saltcake as compared to the original bulk waste. Due to non-ideal crystallization conditions, some soluble components are trapped in occlusions so that decontamination of the salt crystals in the in-tank process is not as effective as expected in a crystallization and crystal washing process designed for this purpose.

Although equilibrium thermodynamic modeling indicates high sodium yields $(>90 \%)$ are theoretically possible by fractional crystallization of Hanford waste, chemical or physical limitations are expected to restrict the extent of sodium salt recovery. Model calculations and testing both show that SST salt cake type wastes are easier to process by fractional crystallization and provide substantially higher yields of decontaminated salt product than do the DST supernates. This is primarily related to the increased concentrations of nitrite, soluble aluminum, and hydroxide in the DST supernates. Selection of waste feeds that are low in these components could significantly improve yield of decontaminated sodium product while allowing less severe/difficult crystallization process conditions. Alternately, the DST supernates could be preprocessed upstream of crystallization to reduce content of these components, e.g., by oxidation of nitrite to nitrate, partial neutralization of hydroxide with nitric acid or carbon dioxide, and/or precipitation of aluminum as gibbsite or low solubility lithium/aluminum compounds.

Depending on relative abundance, sodium sulfate double salts such as burkeite $\left(\mathrm{Na}_{6}\left(\mathrm{SO}_{4}\right)_{2} \mathrm{CO}_{3}\right)$ or the sodium sulfate-fluoride double salt shairerite $\left(\mathrm{Na}_{3} \mathrm{FSO}_{4}\right)$ typically crystallize first upon evaporation of waste. This is usually followed by crystallization of sodium carbonate monohydrate $\left(\mathrm{Na}_{2} \mathrm{CO}_{3} \cdot 1 \mathrm{H}_{2} \mathrm{O}\right)$. Depending on relative abundance, sodium nitrate and/or sodium nitrite may crystallize upon further evaporation. Other sodium salts may crystallize including oxalate, chloride, and acetate if anions are present in significant concentrations. Because solubility is temperature dependent, reducing temperature usually increases equilibrium crystallization yield. The difference in solubility behavior between the sulfate containing salts and other salts provides for a relatively simple scheme for splitting the decontaminated product into a low volume high sulfate stream and a higher volume low sulfate stream. This could be advantageous if there is a desire to reduce sulfate in the feed to the WTP. Equipment for splitting the sulfate between product streams is included in the preliminary equipment and facility design concept developed for this study.

To remove interstitial contamination, mother liquor is separated and washed from the crystal cake. To achieve a high degree of decontamination, effective deliquoring and crystal washing are required.

Centrifugation offers the highest extent of deliquoring, and is the method used in the proposed process concept. To further reduce interstitial contamination, the crystal cake is washed during the centrifugation process to displace contaminated liquor with clean liquid. The extent of decontamination during the wash process depends on the ratio of wash liquid to interstitial liquor. 
In 2004, fractional crystallization was selected for evaluation as a pretreatment process for tank waste to ensure that problematic waste components are diverted preferentially to the Bulk Vitrification facility, while radionuclides (primarily ${ }^{137} \mathrm{Cs}$ and ${ }^{99} \mathrm{Tc}$ ) are diverted to the WTP. In December $2004 \mathrm{CH} 2 \mathrm{M}$ HILL Hanford Group awarded a contract for development of fractional crystallization to a team led by AREVA NC, and including Georgia Institute of Technology, Swenson Technology, Inc. and AREVA NP. A substantial amount of testing and engineering work has subsequently been completed under this ongoing program. A pilot plant has been constructed that is currently undergoing startup testing.

Initial Phase I work was structured to demonstrate that fractional crystallization could be used to pre-treat Hanford tank wastes and to provide data to develop a pilot plant design. Two primary reports were issued to summarize Phase I work completed through the end of 2005: RPP-PLAN-27238, Hanford

Medium/Low Curie Waste Pretreatment Project - Pretreatment Process Plan (CH2M HILL 2006a), and RPP-RPT-27239, Hanford Medium/Low Curie Waste Pretreatment Project - Phase I Laboratory Report (CH2M HILL 2006b).

RPP-PLAN-27238 provides a summary of engineering work through late 2005 , including:

- Basic chemical engineering concepts are reviewed to provide an understanding of unique characteristics of this technology and its application to the Hanford tank wastes.

- Use of a thermodynamic chemical process model, Environmental Simulation Program (ESP) by OLI Systems, Inc, is discussed. The model is used to evaluate the process by investigating waste constituent properties such as Gibbs free energy, solid phases, solution ionic strength, and effects of $\mathrm{pH}$, temperature, water content, etc. The model was used to guide laboratory simulant experiments, and to estimate actual waste behavior in process equipment.

- Flow sheet development and concept selection work is discussed.

- A pre-conceptual design concept is proposed. The report includes preliminary process definition, equipment sizing, facility layout, and construction cost estimates for producing a nominal $5 \mathrm{gpm}$ of decontaminated product to the supplemental immobilization (BV) facility.

- Implementation plans, schedules, and life cycle costs are provided for a system to be deployed near the proposed demonstration bulk vitrification system in the Hanford $200 \mathrm{~W}$ area.

\subsubsection{Process Description}

A two-stage crystallization approach has been selected for the IPS down selection evaluation, in which the partially decontaminated sodium salt product from the first stage is re-dissolved and fed to a second stage crystallizer. The second stage provides additional decontamination of the product and appears likely to be needed to meet the relatively low ${ }^{137} \mathrm{Cs}$ specification for processing DST supernates to meet WTP requirements. Need for the second stage should be determined by the pilot plant testing that is currently in progress. Wash liquor from crystal decontamination and the purge stream from the second stage crystallizer are recycled internally to maximize net yield of decontaminated product.

Figure 2-3 and Figure 2-4 show the process flow diagrams for the first and second crystallization stages respectively. Tank waste is first filtered in a unit operation separate from fractional crystallization to remove suspended solids (see Section 5). Filtrate flows to the Feed Receipt Tank, which provides buffer storage capacity. Waste feed (Stream 4) is transferred continuously to the first stage crystallizer to maintain stable steady-state operating conditions. The crystallizer is operated under vacuum (typically 0.035 to 0.1 atmospheres absolute) to maintain boiling temperatures in the 40 to $60^{\circ} \mathrm{C}$ range needed for crystallization. A relatively large recirculation stream flows from the bottom of the crystallizer through the steam-heated reboiler, which provides heat for water evaporation. 
A slurry containing crystals and liquor is drawn off the crystallizer and pumped (Stream 19) to a centrifuge for separation of liquor from the crystals followed by crystal washing to remove residual contamination. A concentrated (nominal 10 mole/liter Sodium) salt solution is used for washing to avoid significant dissolution of crystals in the centrifuge. Washed crystals are discharged to a dissolver tank where water is added and the slurry is heated to re-dissolve most of the sodium salts. A small amount of low solubility and/or slow dissolving salts such as oxalate, sulfate, and burkeite $\left(\mathrm{Na}_{6}\left(\mathrm{SO}_{4}\right)_{2} \mathrm{CO}_{3}\right)$, may remain as suspended solids in the dissolver product. Part of the dissolver product is recycled to the centrifuge as wash solution to decontaminate the crystal cake.

To eliminate residual fine crystals that could reduce crystal cake permeability the dissolver product is first processed through a filter. Filtrate (Stream 10) is recycled for washing crystals in the centrifuge. The non-filtrate stream from the filter contains about half the liquid and all the residual solids. A small amount of additional dilution water may be added as it exits the filter to help dissolved residual solids and the combined stream (20) is then transferred to the second stage crystallizer.

A portion of the liquor stream from the centrifuge is recycled to the crystallizer, while the remainder (Stream 13) is purged to the Cesium Product Tank. It may be diluted with water in the Cesium Product Tank if needed prior to return to a DST. Vapor from the crystallizer (Stream 5) flows through a deentrainer and demister in the top of the crystallizer to remove entrained contaminants, and then flows to the first stage condenser where the bulk of the water vapor is condensed. Remaining water vapor and non-condensable gases then flow through two steam jet eductors with condensers that maintain vacuum on the crystallizer. Vent gas is filtered prior to discharge to the environment.

Process condensate is collected from the primary condenser and steam jets and is used for dissolution and dilution of product as needed. Surplus process condensate (Stream 30) is transferred to an external treatment facility, assumed to be the Effluent Treatment Facility. Efficient de-entrainment is required to remove waste particles from steam generated in the crystallizer. To assure Effluent Treatment Facility acceptance requirements for ${ }^{137} \mathrm{Cs}$ are met a minimum decontamination factor of $3 \times 10^{5}$ must be specified as a design requirement for the crystallizer (defined as the ratio of cesium concentration in the crystallizer concentrate divided by the concentration in the condensate). Process condensate will have trace amounts of other contaminants expected to be at levels similar to process condensate from the 242-A Evaporator. 
Figure 2-3 - Fractional Crystallization First Stage Process Flow Diagram

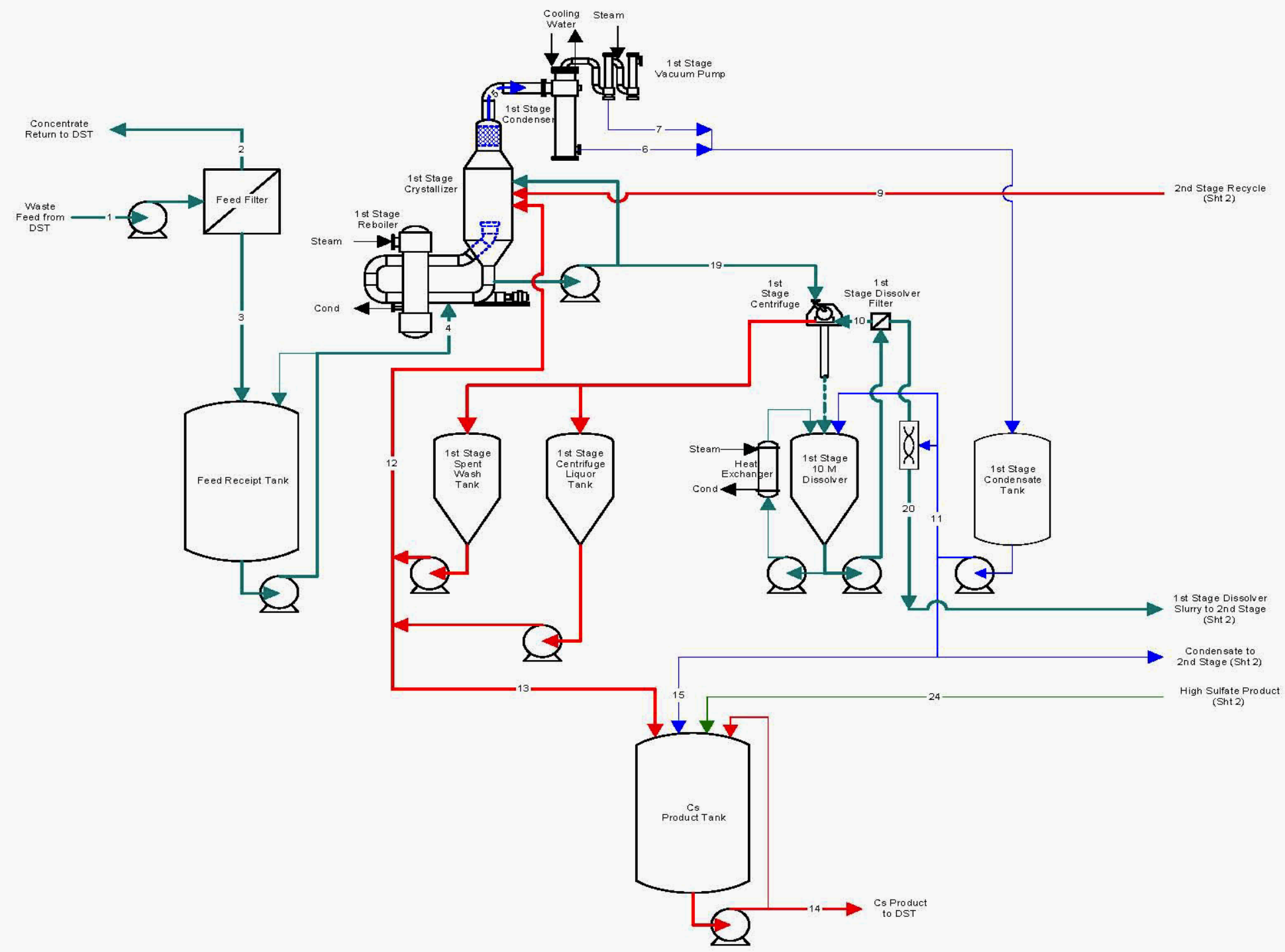


Figure 2-4 - Fractional Crystallization Second Stage Process Flow Diagram

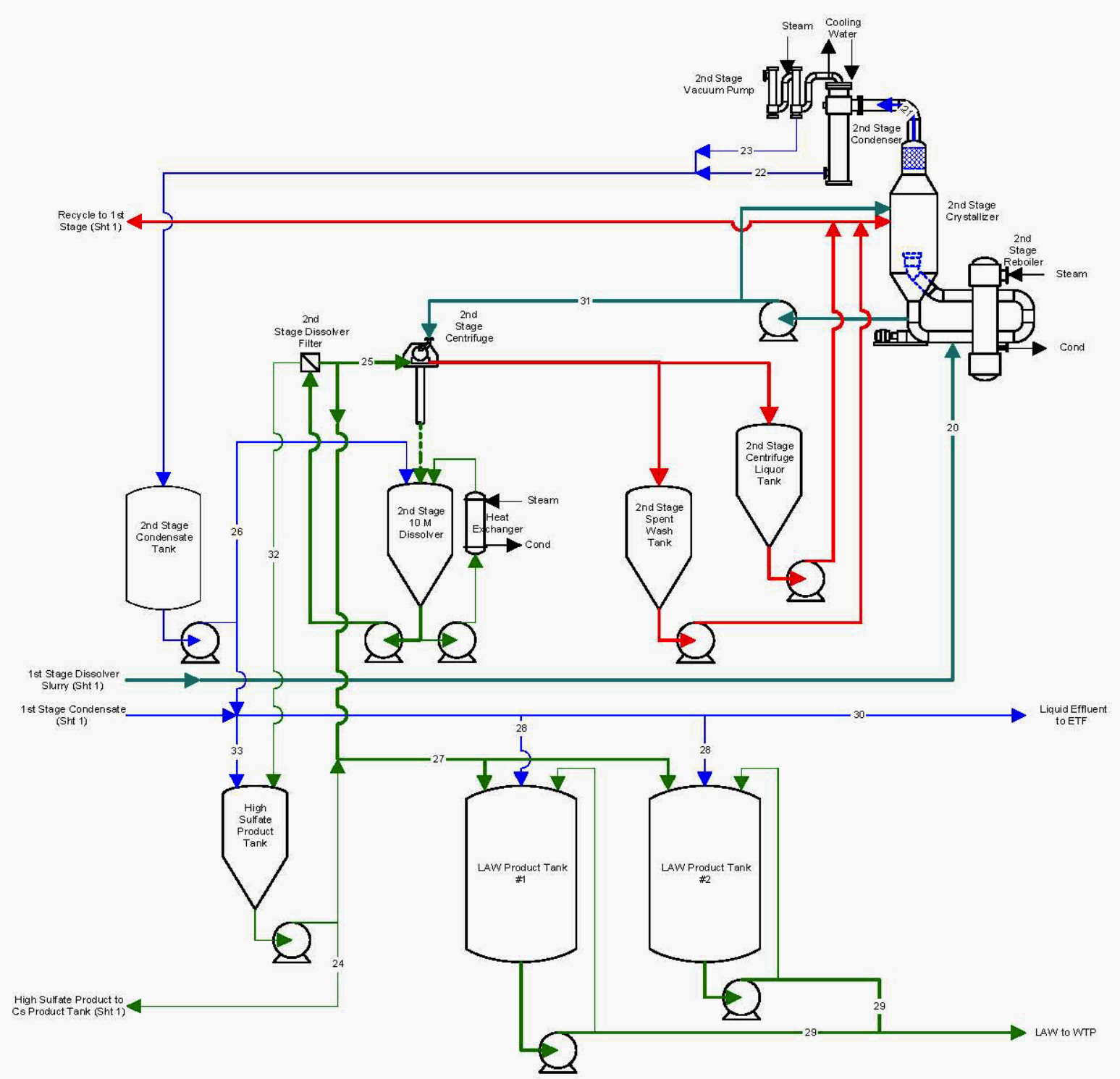


The second stage crystallizer system operates essentially the same as the first stage, except that feed comes from the first stage dissolver (Stream 20), the purge (Stream 9) is recycled to the first stage crystallizer, and the second stage dissolver and product filtration has the additional function of controlling sulfate in the LAW product and is therefore sized and operated somewhat differently than the first stage. Dilution water addition to the dissolver is controlled so that most of the sulfate remains as undissolved crystals. Most of the liquid is passed through the filter media ( $90 \%$ or more versus about $50 \%$ for the first stage). This produces concentrated high-sulfate slurry and a low sulfate filtrate. About half the filtrate (stream 25) is recycled for washing filter cake in the centrifuge. Balance of the filtrate represents the primary LAW product, and is transferred to the LAW Product Tanks. The high sulfate stream from the filter is collected in a separate High Sulfate Product Tank and may be split between the LAW product tank and the cesium product tank in order to control the amount of sulfate in the LAW product. The combined low sulfate and high sulfate LAW products (Stream 27) are accumulated in the LAW product tanks and may be diluted further with water as needed prior to transfer to WTP (Stream 29).

Because the first stage delivers a concentrated product, the second stage crystallizer evaporation duty is significantly lower than the first stage. Operating conditions and stream properties in the second stage may also be significantly different because of the reduced concentration of low solubility waste components.

The process is controlled to maintain steady-state operation of the entire crystallizer system. Process variables, including temperatures, pressures, crystallizer slurry density, flow rates, tank levels, etc. are measured and controlled to maintain process variables at set point values. Routine sampling and analysis is not expected to be needed for process control, assuming the feed has been characterized in advance for each batch. Sampling of selected process streams is performed occasionally on an as-needed basis to support optimization, troubleshooting, and regulatory compliance documentation. Efficiency of ${ }^{137} \mathrm{Cs}$ decontamination in each crystallizer stage is monitored in real time by measuring gamma radiation dose rates of the dissolver tank and/or dissolver product. Amount of dissolved product recycled for washing centrifuge cake is adjusted as needed to assure target ${ }^{137} \mathrm{Cs}$ decontamination is achieved. Offspecification product is recycled to the crystallizer feed tank. Draining and flushing capability is provided to reduce personnel dose rate and allow certain maintenance functions to be performed. Capability is provided to empty the crystallizer contents back to the feed tank during unplanned shutdowns.

The control system allows manual operation during startup and shutdown, but operates in an automatic mode during normal conditions. Initially, the feed tank and crystallizer are charged with fresh feed solution and vacuum is drawn on the system before feed is heated in the reboiler. Additional feed is added as needed to maintain constant liquid level in the crystallizer as water is evaporated.

\subsubsection{Caustic-Side Solvent Extraction}

Since its inception the nuclear industry has successfully used solvent extraction to separate radionuclides. The experience base includes exposing various organic solvents to high radiation fields without experiencing catastrophic degradation rates. The typical key to solvent extraction's effectiveness is development of an organic solvent that is sufficiently selective in adsorption of the targeted radionuclide(s). 


\subsubsection{Technology Development Summary}

ORNL has developed a solvent that is relatively selective for cesium removal from an alkaline solution. The solvent consists of calix [4] arene-bis(tert-octylbenzo-crown-6) extractant (BOBCalixC6) dissolved in an inert hydrocarbon matrix (Isopar ${ }^{\circledR} \mathrm{L}$ ). The solvent contains a modifier, which is an alkyl aryl polyether, to keep the extractant dissolved in the solvent and increase its ability to absorb cesium in the extraction section. The modifier is 1-(2,2,3,3-tetrafluoropropoxy)-3-(4-sec-butylphenoxy)-2-propanol and is called Cs-7SB. The solvent contains a suppressant, tri-octyl-amine (TOA), which inhibits the effects of anionic organic impurities and improves the back-extraction of cesium from the solvent in the stripping section.

This development resulted in efforts to deploy the Caustic-Side Solvent Extraction (CSSX) process at the Savannah River Site to extract cesium from alkaline supernate and dissolved salt cake wastes. The Savannah River Site's Modular CSSX Unit (MCU) began processing tank waste in May 2008.

Recent CSSX technology development work presented in "Alternatives to Nitric Acid Stripping in the Caustic-Side Solvent Extraction (CSSX) Process for Cesium Removal from Alkaline High-Level Waste" (Delmau et al. 2008) proposed flow sheet modifications that would increase the efficiency of cesium stripping. This work recommends replacing the nitric acid scrubbing and stripping solutions with $0.1 \underline{\mathrm{M}}$ $\mathrm{NaOH}$ and $0.01 \underline{\mathrm{M}}$ boric acid $\left(\mathrm{H}_{3} \mathrm{BO}_{3}\right)$, respectively. Also proposed is replacing BOBCalixC6 with a more soluble analog extractant, calyx [4]arene-bis (2-ethylhexylbenzo-18crown-6) (BEHBCalixC6) and TOA with N,N'-bis(cyclohexyl)-N"-isotridecylguanidine (LIX 79). These modifications are expected to increase CSSX performance for the case where it's applied to high potassium-content feeds, such as those at Hanford. However, the potential performance increase is not incorporated into this study because a considerable amount of additional development would be required before these modifications could be adopted as a basis for design.

While Delmau et al. 2008 has recently proposed modifying the solvent composition to optimize it for high potassium-content feeds, the $\mathrm{BOBCalix} C 6$ solvent composition will perform adequately with some Hanford Site feed batches. The chemical and physical properties of this solvent are extensively reported in ORNL/TM-2002/190, Caustic-Side Solvent Extraction: Chemical and Physical Properties of the Optimized Solvent. Measured physical properties include density, viscosity, and thermal stability.

Of particular value is distribution of minor organic and inorganic components that are generally not reported in the extensive body of CSSX literature. Based on mass balance data presented in ORNL/TM-2002/190 about $0.023 \%$ of $\mathrm{Na}$ and $12.2 \%$ of $\mathrm{K}$ in the waste feed are extracted into the solvent. After the second scrub stage only $1.05 \%$ of the $\mathrm{Na}$ and $0.218 \%$ of the $\mathrm{K}$ remain in the solvent.

The conclusion reported in ORNL/TM-2002/190 was that solvent losses through degradation were negligible. Entrainment of solvent in the aqueous streams was the dominant loss mechanism. Test results reported in WSRC-TR-2005-00182, Examination of Organic Carryover from 2-cm Contactors to Support the Modular CSSX Unit, indicated that organic carryover after decanting was bounded by $417 \mathrm{ppm}$ of Isopar L. Therefore, solvent replenishment should be much less that one process inventory (180 to 390 gal) per year.

Since the late 1990's, SRS has been developing the CSSX process in conjunction with ORNL and Argonne National Laboratory (ANL). In 2002 the results of "hot" laboratory-scale testing were reported in WSRC-TR-2002-00243, High Level Waste Demonstration of the Caustic-Side Solvent Extraction Process with Optimized Solvent in the 2-cm Centrifugal Contactor Apparatus using Tank 37H/44F Supernate, and WSRC-TR-2002-00307, Demonstration of Caustic-Side Solvent Extraction with Optimized Solvent in the 2-cm Centrifugal Contactor Apparatus using Dissolved Salt Cake from Tank 
37H. These test using actual tank waste verified that the CSSX process could yield sufficient cesium removal that the decontaminated waste was suitable for immobilization in grout and onsite disposal. The results were sufficiently positive that efforts were initiated to take the CSSX process to a full-scale production facility.

The heat imparted by the contactors into the fluids was estimated by ANL-00/31, Temperature Management of Centrifugal Contactors for Caustic-Side Solvent Extraction of Cesium from Tank Waste. The cesium distribution coefficient $\left(\mathrm{D}_{\mathrm{Cs}}\right)$ is a strong function of temperature. A lower fluid temperature is favorable to extraction while a higher temperature more conducive to stripping. For this reason $25^{\circ} \mathrm{C}$ or less is the preferred operating temperature for extraction. ANL-00/31 determined that the contactor motor was the dominant heat source, contributing more than $90 \%$ of the overall heat load.

Efforts have also been directed towards understanding the influence of waste components on CSSX performance. WSRC-TR-2005-00258 investigated 12 waste feed components (i.e., $\mathrm{Na}^{+}, \mathrm{K}^{+}, \mathrm{Cs}^{+}, \mathrm{OH}^{-}$, $\mathrm{NO}_{3}{ }^{-}, \mathrm{NO}_{2}{ }^{-}, \mathrm{Cl}, \mathrm{F}^{-}, \mathrm{SO}_{4}{ }^{-2}, \mathrm{PO}_{4}{ }^{-3}, \mathrm{CO}_{3}{ }^{-2}$, and $\mathrm{AlO}_{2}{ }^{-}$) and determined that potassium exhibits the strongest detrimental influence on $\mathrm{D}_{\mathrm{Cs}}$ for extraction. A neural network model was also developed to predict $\mathrm{D}_{\mathrm{Cs}}$ for extraction given a waste feed composition. This correlation predicted $\mathrm{D}_{\mathrm{Cs}}$ values within $15 \%$ of those predicted by a more rigorous, but complex model termed SXFIT.

In 2004 a conceptual design for the MCU was completed and documented in ORNL/TM-2004/59, Conceptual Design of a Simplified Skid-Mounted Caustic-Side Solvent Extraction Process for Removal of Cesium for Savannah River Site High-Level Waste. The throughput of the MCU process is similar to that required for IPS ( 6 to $8 \mathrm{gpm}$ ). However, the MCU is design to only achieve a decontamination factor ( $\mathrm{Ci}{ }^{137} \mathrm{Cs}$ in waste feed divided by $\mathrm{Ci}^{137} \mathrm{Cs}$ in decontaminated product) of 12 versus the IPS requirement of 1,000 to 4,000 . This difference in decontamination factor directly translates into the number of contactors that must be included in the respective flow sheets (seven in the extraction section of the MCU versus 14 in the IPS).

In 2007 the Savannah River Site completed construction and assembly of the MCU facility. Cold testing was conducted after assembly to assess process performance. Conclusions derived from the test are presented in WSRC-STI-2007-00580, Full-Scale Testing of a Caustic Side Solvent Extraction System to Remove Cesium from Savannah River Site Waste. Overall, test results were in agreement with expectations.

Work is underway at Oak Ridge National Laboratory to test CSSX using simulants of the eight IPS feed batches. Reported results are expected at the end of May 2008. Preliminary results indicate that the number of required equilibrium stages range from 13 to 105 using the BOBCalixC6 solvent if the organic to aqueous flow rate ratio $(\mathrm{O} / \mathrm{A})$ is fixed at 0.3 . Using the BEHBCalixC6 solvent the required equilibrium stages are reduced to 6 to 10 for an $\mathrm{O} / \mathrm{A}$ of 0.3 .

\subsubsection{Process Description}

Figure 2-5 depicts the CSSX flow sheet proposed for the IPS. This flow sheet is functionally equivalent to that implemented in the Savannah River Site's MCU, which was initially developed by ANL. The flow sheet represents a continuous process that includes multiple steps.

The first step is adjustment of the waste feed with caustic and water to yield a $6 \underline{\mathrm{M}}$ sodium product. This adjustment is required to preclude precipitation of $\mathrm{Al}(\mathrm{OH})_{4}$ when the feed is cooled to $25^{\circ} \mathrm{C}$. Operation of the extraction section at $25^{\circ} \mathrm{C}$ is required to yield the best cesium removal performance. 
In the extraction section the aqueous feed stream is mixed with the organic solvent, which is immiscible with the aqueous phase. During this contacting cesium (the target component) undergoes transfer from the aqueous stream to the organic stream. Other non-target components (predominately sodium and potassium) are also absorbed into the organic phase during extraction. However, most non-target components remain in the aqueous feed stream. Following extraction the feed stream (termed raffinate) is depleted in cesium to the desired level and becomes acceptable for transfer to the WTP as LAW.

After extraction the cesium-loaded organic (termed the extract) is routed to the scrubbing operation where it is contacted with a dilute $(0.05 \underline{\mathrm{M}})$ nitric acid solution. The purpose of scrubbing is to remove the $\mathrm{Na}$ and $\mathrm{K}$ impurities from the extract, prior to cesium stripping. The scrubbed organic then proceeds to the stripping operation.

In a deviation from the MCU flow sheet the aqueous stream is routed to Cesium Product Tank. For the CSSX applications at Hanford there is little benefit in returning to the LAW product the relatively small quantities of $\mathrm{Na}$ and $\mathrm{K}$ separated during extraction. To compensate for nitric acid neutralization mixing the aqueous scrubbing solution with waste feed prior to extraction (as is done in the MCU flow sheet) would require addition of even more caustic than that needed to preclude $\mathrm{Al}(\mathrm{OH})_{4}$ precipitation. The increase in aqueous flow rate to extraction can also result in a higher organic flow rate and/or greater number of contactors. Therefore, for CSSX applications at Hanford it's preferable to blend spent scrubbing solution with the cesium product.

During stripping the solvent is again contacted with a dilute $(0.001 \underline{\mathrm{M}})$ nitric acid solution which causes most of the cesium ions to transfer from the organic to the aqueous phase. The cesium-loaded aqueous stream is collected, chemically adjusted to meet tank farm acceptance specifications, and subsequently transferred to a DST.

Following stripping the cesium-depleted solvent is routed to the washing operation where it is contacted with a dilute $(0.01 \underline{\mathrm{M}})$ caustic solution. The washing step serves to remove trace organic impurities that are the result of solvent degradation. The organic stream is recycled to extraction after washing to begin the process anew.

In a second deviation from the MCU flow sheet the caustic wash stream is routed to Cesium Product Tank; the MCU flow sheet sends this stream to the LAW Product Tank. For the CSSX applications at Hanford the cesium product must be adjusted with caustic and sodium nitrite to satisfy the tank farm corrosion specification. Sending the aqueous stream to the Cesium Product Tank Mixing offsets some sodium that would otherwise have to be added for chemical adjustment.

The solvent extraction process (i.e., extraction, scrubbing, stripping, and washing operations) is carried out using a series of centrifugal contactors. Figure 2-6 is a schematic of a contactor and Figure 2-7 is a rendering of a prototypical 2-stage contactor unit. As reported in LWO-SPT-2007-000245, Scale-up of Caustic-Side Solvent Extraction Process for Removal of Cesium at Savannah River Site, full-scale contactor performance was confirmed to be sufficient to support MCU design basis requirements.

The annular centrifugal contactor was developed at ANL in the early 1970 s for carrying out solvent extraction operations required in the nuclear industry. Costner Industries Nevada Corporation has been supplying commercially-available units to the Savannah River Site for their MCU project. In stage-wise solvent extraction, two immiscible liquids are contacted and then separated at each stage. For continuous countercurrent operation, the immiscible liquids move in opposite directions as they flow from stage to stage. 
Figure 2-5 - Caustic-Side Solvent Extraction Process Flow Diagram

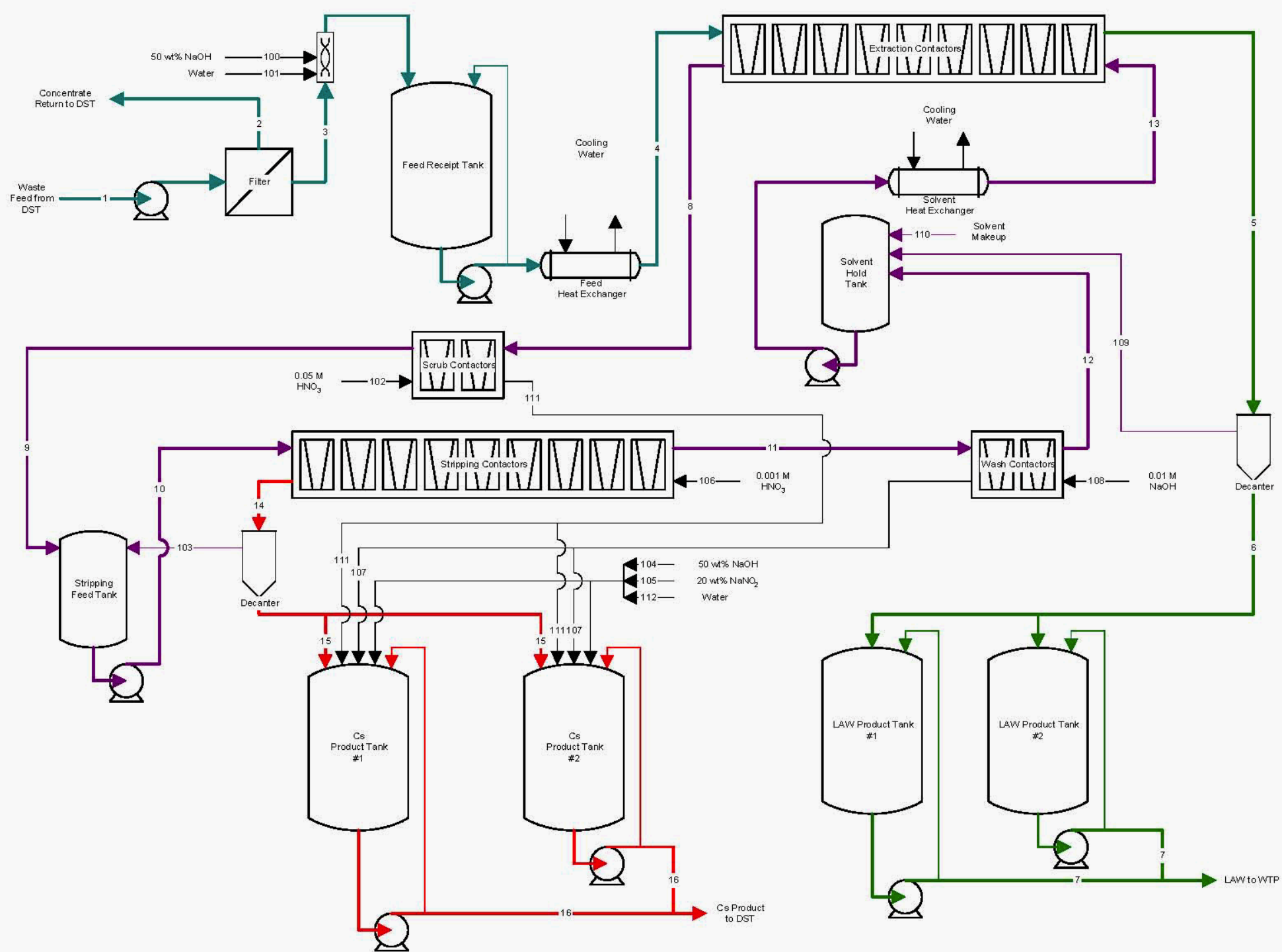




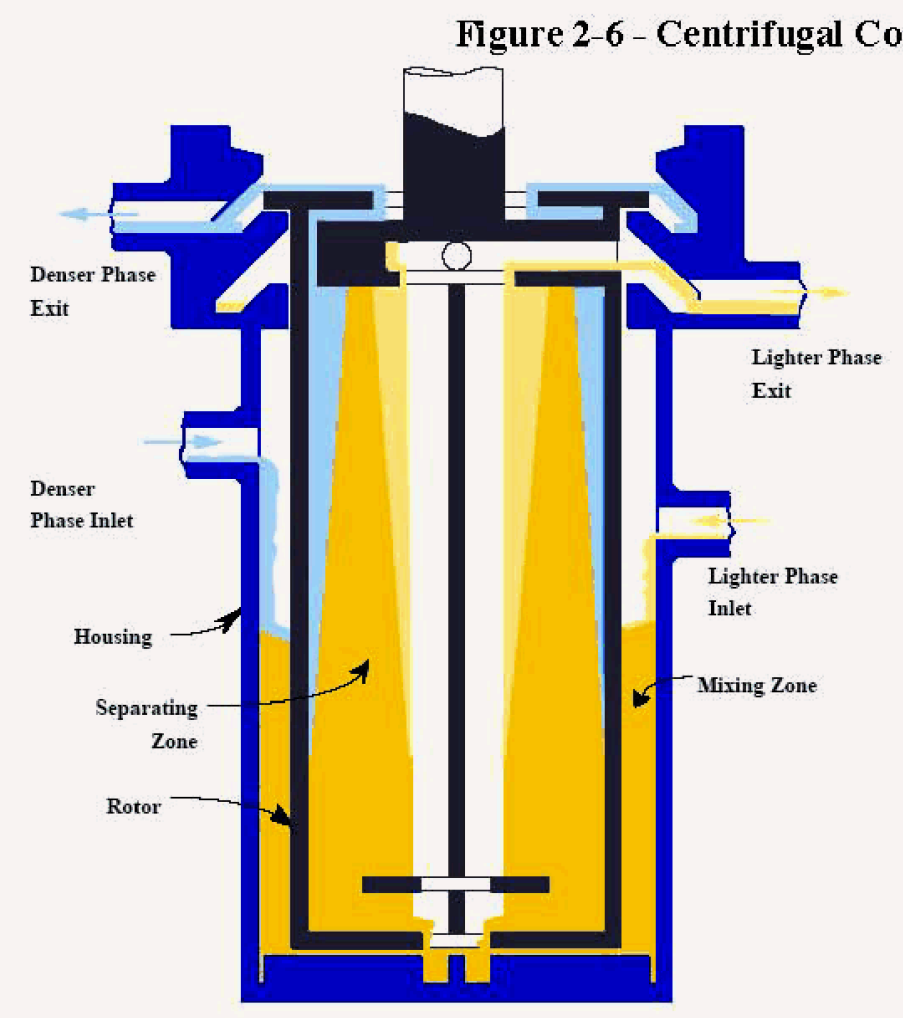

Figure 2-7 - Prototypical 2-Stage Contactor Unit

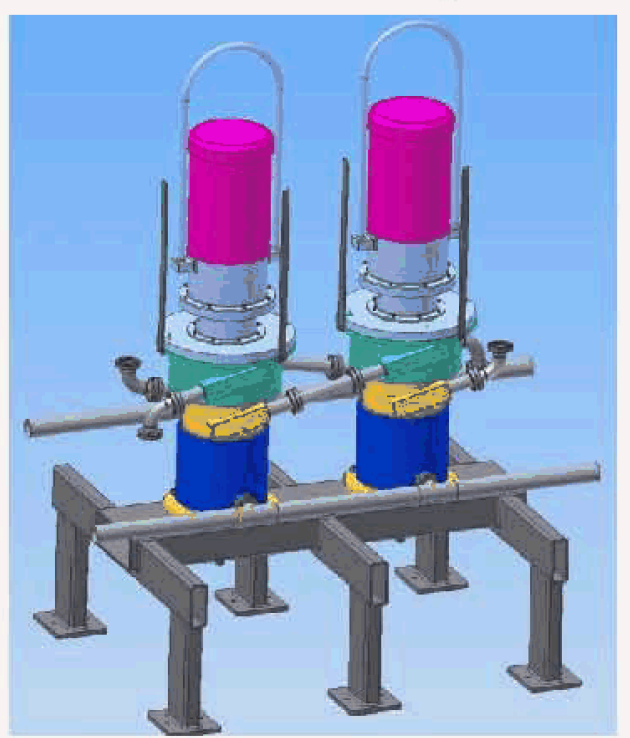


The entering immiscible liquids are mixed in the annular region outside the spinning rotor. The mixture is disengaged by the centrifugal force in the separating zone of the rotor. The separated liquids are then forced to move out separate rotor exits by the two rotor weirs (the upper weir for the more-dense phase, the lower weir for the less-dense phase), where they are captured by separate collector rings near the top of the contactor housing. The liquids then flow by gravity to the appropriate next stage. A motor located above the rotor spins the rotor.

The efficiency to which the CSSX process extracts cesium from the feed stream is directly related to $\mathrm{D}_{\mathrm{Cs}}$, which is related to specific feed composition being processed. WSRC-TR-2005-00258, Waste and Solvent Composition Limits for Modular Caustic-Side Solvent Extraction Unit (MCU), identified a correlation that reasonably predicts $\mathrm{D}_{\mathrm{Cs}}$ for a given feed composition.

\subsubsection{Ion Exchange using Spherical Resorcinol Formaldehyde Resin}

\subsubsection{Technology Development Summary}

Ion exchange flow sheet development is primarily based on 24590-WTP-RPT-RT-07-005, Implementation Recommendations for WTP Use of Spherical Resorcinol Formaldehyde Resin as the Primary Cesium Ion Exchange Resin. This document provides a summary of test data that have been accumulated through 2007 to investigate implementation of Spherical Resorcinol-Formaldehyde as the cesium removal resin in WTP Pretreatment Facility ion exchange columns.

The data summarized include:

- Hydraulic performance test results

- Column cesium removal performance test data

- Resin degradation test results

Laboratory-scale column testing ( $\sim 1.5 \mathrm{~cm}$ diameter columns) has been performed using actual waste from two different waste tanks. The remainder of the Spherical Resorcinol-Formaldehyde test experience has been obtained using waste simulants.

Hydraulic performance test experience was obtained using bench-scale and pilot-scale equipment with column diameters of 3-inches and 24 -inches, respectively. The pilot-scale equipment approaches the column size projected to be required for the IPS implementation of ion exchange technology ( $\sim 34$-inch diameter column).

Data to predict cesium removal performance by ion exchange columns are obtained from laboratory scale testing ( 1.5 to $2 \mathrm{~cm}$ diameter columns). Cesium removal performance tests were completed using the pilot-scale equipment. However, the pilot-scale tests using feed materials spiked with cesium were limited by the quantity of chemicals that could be handled in the test facility. Cesium in the column effluent was at the detection limit of analytical techniques throughout the loading cycle. Therefore, a cesium breakthrough curve could not be constructed based on data obtained from the larger scale equipment.

Hydraulic testing on a pilot-scale cycled a resin bed through 17 load, rinse, elution, and regeneration cycles to investigate the impact of chemical degradation on resin bed performance. The impact of chemical degradation on cesium removal performance was evaluated by comparing cesium breakthrough curves developed using resin samples of the pilot scale resin bed after cycling in a laboratory-scale column with fresh resin test results. Radiation degradation was evaluated by comparing the equilibrium batch distribution data of resin samples irradiated up to $100 \mathrm{Mrad}$ with similar data for unirradiated resin. Actual data investigating the combined effects of chemical and radiation degradation are not available. 
Resin degradation effects must be extrapolated from these test data to approximate the column performance after reuse of resin beds for 30 loading cycles.

\subsubsection{Process Description}

The cesium ion exchange process contains multiple operating phases. Figure 2-8 describes the primary process flow diagram from the Cesium Ion Exchange process. This figure represents the dominant operating configuration used for waste processing by ion exchange. Filtrate obtained from the selected solids separation system becomes feed to the ion exchange system.

An ion exchange operating condition of $6 \mathrm{M}$ sodium ion was selected for the flow sheet. Limiting the waste sodium ion concentration approximates a limitation of the waste viscosity passing through an ion exchange resin bed to range that has been demonstrated to produce acceptable pressure drops through resin beds at the selected design superficial velocity. In addition, the $6 \mathrm{M}$ sodium ion concentration is within the range of sodium concentrations where ion exchange equilibrium data are available to describe cesium removal performance.

An operating temperature of $25^{\circ} \mathrm{C}$ was also selected as the basis for flow sheet preparation. This operating temperature is consistent with the base operating temperature selected for ion exchange implementation in the WTP. Loading cycle volumes decrease as temperature is increased which increases the number of rinse, elution, and regeneration cycles that must be performed to process a unit volume of waste. In addition, resin degradation can increase significantly in the nitric acid eluant at temperatures above $45^{\circ} \mathrm{C}$. Therefore, operating at a constant temperature of $25^{\circ} \mathrm{C}$ minimizes the potential for excessive resin degradation.

Supernate is diluted with caustic to $6 \mathrm{M} \mathrm{Na}$ so that aluminum solids are not precipitated when cooled to $25^{\circ} \mathrm{C}$, prior to storage in the ion exchange column feed tank. The dilution supports the selected operating conditions while preventing formation of solids that may foul resin bed during waste processing.

The ion exchange column feed is accumulated in a lag storage vessel that is used as a pump tank. This provides hold up between the solids separation and ion exchange systems so that filtered LAW can continue to be generated when the ion exchange column is not operating for short time periods (e.g., during period rinse, elution, and regeneration cycles).

The flow sheet is based on use of the spherical form of Resorcinol-Formaldehyde as the resin bed. Two columns in series are included for processing waste during the cesium loading cycle, a lead column and a polishing column. The two-column system allows process monitoring of the stream between the two columns to determine when the columns should be eluted to ensure adequate cesium removal is obtained. Waste feed is processed through the two ion exchange columns to remove cesium during the loading cycle. After passing through the two columns, the LAW is collected in one of two tanks prior to transfer out of the IPS and to the LAW Vitrification facility. 
Figure 2-8 - Ion Exchange System Process Flow Diagram

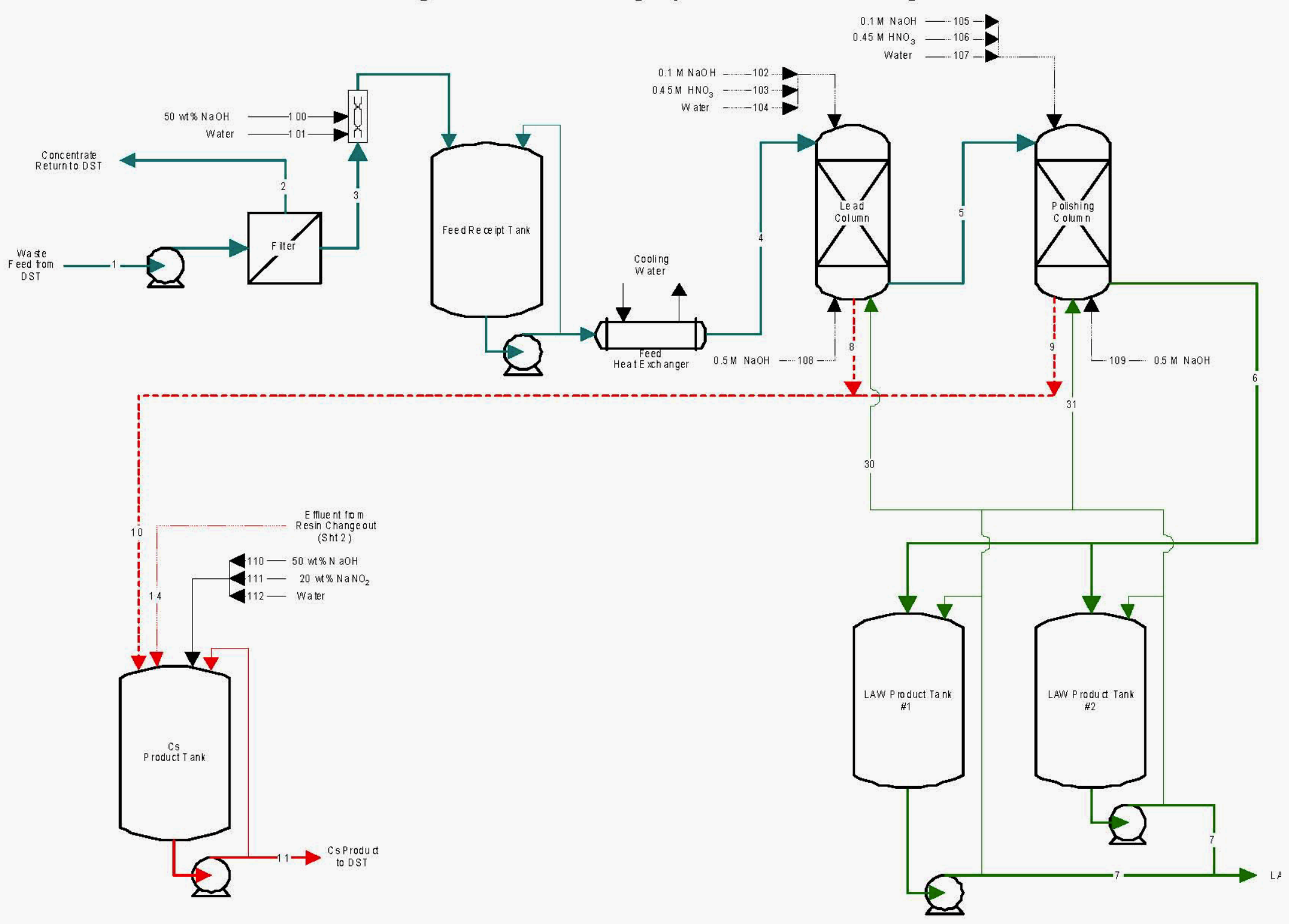


The two-column ion exchange system operates in a semi-continuous operating mode. Most of the operating time is spent in the loading cycle, where cesium in waste supernate passing through the resin bed is adsorbed by the resin. The volume of waste processed by a loading cycle is determined by the concentration of cesium observed in the Treated LAW product. The loading cycle is stopped when the observed cesium concentration leaving the lead column is one-half the cesium concentration in feed to the lead column (a short hand notation for this is $\mathrm{C} / \mathrm{C}_{0}=0.5$, where $\mathrm{C}$ is the cesium molar concentration in the column effluent and $\mathrm{C}_{0}$ is the cesium molar concentration in the column feed). For this study, the loading cycle duration has been defined as the volume of waste processed when the time averaged concentration of waste leaving the Polishing column is equivalent to $1.68 \times 10^{-5} \mathrm{Ci}^{137} \mathrm{Cs} / \mathrm{g}-\mathrm{mol} \mathrm{Na}$ to produce a $\mathrm{LAW}$ product that is comparable to the other separation alternatives.

When process monitoring indicates that the end of the loading cycle has been reached, waste feed to the ion exchange columns is stopped. Both lead and polishing columns are then rinsed, eluted to remove cesium from the resin, and regenerated, allowing the ion exchange columns to be reused to treat additional waste. The rinse, elution, and regeneration activities are composed of a series of operating cycles summarized as follows:

- Feed Displacement Cycle - Displaces waste from the column liquid hold up using a solution of $0.1 \mathrm{M} \mathrm{NaOH}$ (dilute caustic is used for displacement to avoid precipitating aluminum solids in the column)

- Pre-Elution Rinse Cycle - Displaces residual caustic from the column liquid hold up using water (minimizes acid-base neutralization heat generated when acid added to column during elution)

- Elution Cycle - Elutes cesium from the resin bed using a solution of $0.45 \mathrm{M} \mathrm{HNO}_{3}$

- Post-Elution Rinse Cycle - Displaces residual acid from the column liquid hold up using water (minimizes acid-base neutralization heat generated when caustic added to the column during regeneration)

- Regeneration Cycle - Converts resin from the H-form to the Na-form prior to introducing waste into the column. Resin beads also expand when converted to the Na-form. Therefore, regeneration is performed upflow, under bed fluidizing conditions, to minimize the potential for generating stresses in the resin bed that reduce permeability and increase bed pressure drop

- Regenerant Displacement Cycle - Displaces spent regeneration solution from the column using a small volume of LAW that was produced during the loading cycle. Regenerant displacement is also performed upflow, under fluidizing conditions, to complete the resin bead expansion at waste solution ionic strength and displace the low density regenerant with an upflow of high density waste such that the resin bed is not disturbed by waste addition at the start of the loading cycle

Upon completion of the regeneration cycle, the two ion exchange columns are ready to resume operation in the loading cycle configuration and remove cesium from additional waste supernate. Column effluents, including rinse, eluate, and regenerant, from both ion exchange columns are directed to a Cesium Product Tank where the solutions are chemically adjusted to conform with tank farm corrosion criteria and returned to the DSTs for storage.

The resin used for treating waste supernate slowly degrades as solutions are passed through the columns. Some chemical and radiolytic degradation has been observed during laboratory testing. Chemical oxidation occurs due to the presence of dissolved oxygen in waste, rinse, elution, and regeneration solutions that pass through the resin bed. Radiolytic degradation is assumed to occur primarily during the loading cycle, as ${ }^{137} \mathrm{Cs}$ accumulates in the columns. 
Resin degradation reduces the capacity of resin to adsorb cesium from the waste, resulting in a shortened loading cycle as resin is reused. At some point, the loading cycle duration becomes short compared to the rinse, elution, and regeneration cycle duration and the resin bed replaced in order to maintain the design throughput rate. Figure 2-9 indicates the process flow diagram for replacing resin. Resin is removed from each column using a fluidizing flow of water with the resultant resin/fluid slurry transferred to spent resin accumulation tank.

The resin replacement cycles are projected to be performed two to three times per year of operation. Prior to performing the resin replacement cycle, fresh resin is prepared in a make-up tank. Resin is received in 55 -gal drums in the H-form. The resin is converted to the Na-form prior to addition to a column by transferring fresh resin from the drums into a solution of $\mathrm{NaOH}$ to produce slurry containing $28 \%$ resin by volume. Two resin makeup tanks have been included in the ion exchange system configuration (one for each column) to minimize down time spent for resin bed replacement and control the volume of resin added to an individual column. After spent resin has been removed from a column, the fresh resin slurry is transferred by gravity draining from the makeup tank to the column.

Liquid holdup in the empty column is displaced to the spent resin accumulation tank during fresh resin addition to the column. Once the fresh resin has been added to both columns, LAW is used to displace the transfer solution and the columns are ready to resume waste processing.

Spent resin is removed from the system as a solid waste. The approach selected for preparing spent resin for solid waste load out is similar to the approach developed for the WTP Pretreatment facility. The accumulated spent resin slurry is transferred from the spent resin tank into a cask that contains an internal screen. The cask is sized to contain resin from two columns. The internal screen confines resin within the cask as the slurry passes through the cask, with the transfer liquid combined with other process effluents in the Cesium Product Tank that are returned to DSTs.

After completion of the slurry transfer, emptying the spent resin accumulation tank, the disposal cask contains a combination of resin solids and transfer liquid holdup (primarily water). Free liquid remaining in the cask must be removed to satisfy solid waste disposal criteria. Dewatering is accomplished by bubbling heated air through the disposal cask using the same input leg as used for introducing the spent resin slurry. It is estimated that an air stream of $100 \mathrm{SCFM}$, heated to $75^{\circ} \mathrm{C}$, will complete spent resin dewatering in approximately 7 days. After dewatering, the cask penetrations are sealed and the cask transferred out of the ion exchange facility for disposal. 
Figure 2-9 - Resin Replacement Process Flow Diagram

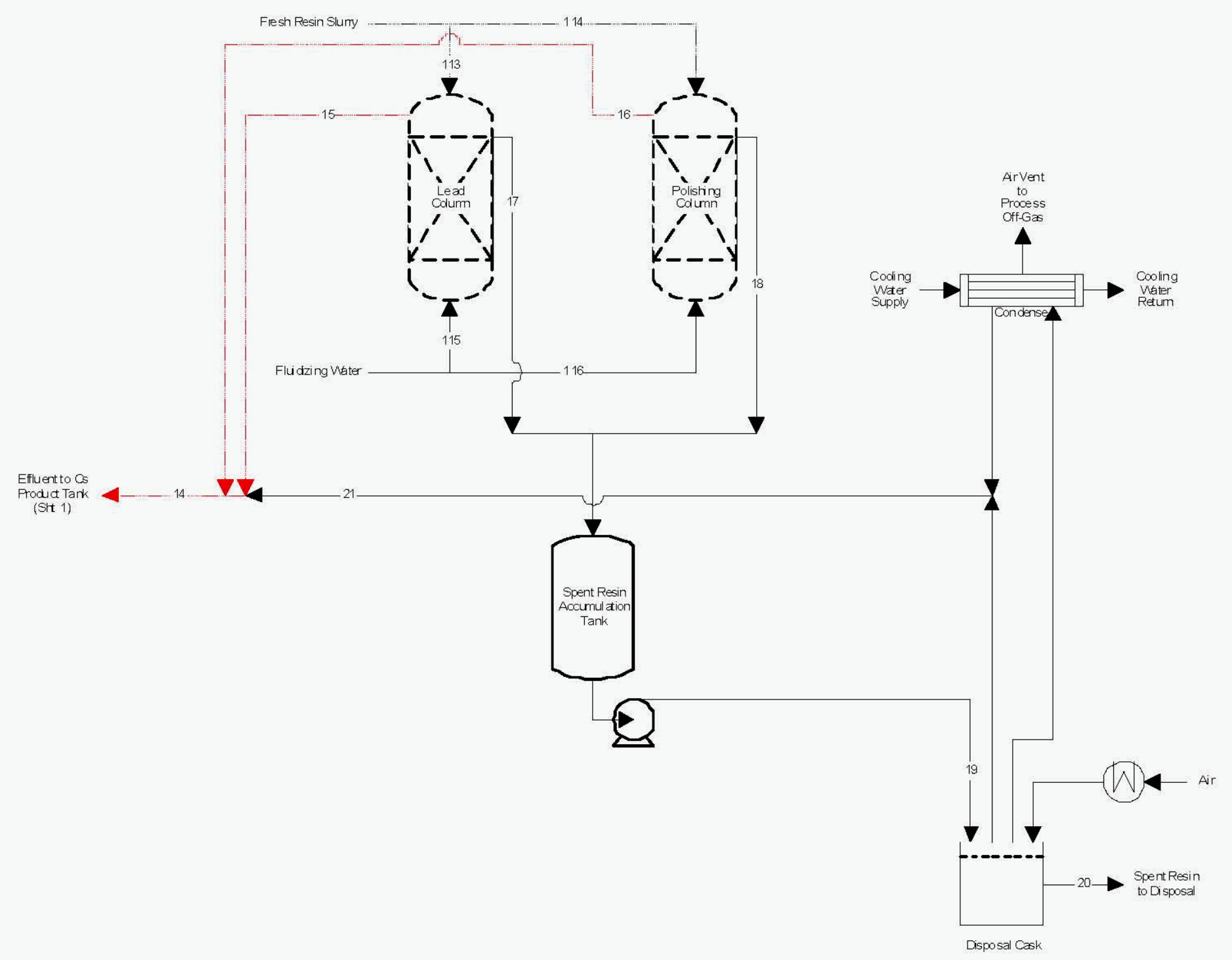




\subsection{ENTRAINED SOLIDS SEPARATION ASSESSMENTS}

Two technologies (cross-flow filtration and rotary micro-filtration) have been identified for use within the IPS to remove entrained solids that potentially contain ${ }^{90} \mathrm{Sr}$ and transuranic elements. The basic function achieved by these two technologies is to remove entrained solids from the DST feed stream prior to cesium removal. Removed entrained solids are returned to the DSTs, while the filtrate is processed further to remove cesium prior to vitrification in the LAW facility. This section summarizes the Assessment Form information details for each decision line, compiled in RPP-RPT-37741 (CH2M HILL 2008d). Individual Assessment Form numbers are noted below with the Assessment form designator, e.g., CF-1.1.1; no specific reference back to RPP-RPT-37741 (CH2M HILL 2008d) is included with this designation, but is implied.

\subsection{SAFETY}

Three measures, Process Safety, Criticality Safety, and Industrial Safety and Hygiene have been identified for this Criterion. The safety assessment discussion is provided below.

\subsubsection{Process Safety}

Eight process safety definitions (Attachment A) have been identified to assess overall process impacts of these two solids/liquid separation technologies on various process safety aspects including; nuclear safety, chemical safety, fire safety, and operational safety. These safety issues are covered by looking at various aspects of Materials at Risk (MAR), process stability, chemical reactivity, flammability, and operational hazards. For two filtration technologies these items are discussed below.

\subsubsection{Quantity of Material at Risk (MAR)}

This process safety definition evaluates whether there are any differences in the quantities of MAR between the two options. Less material at risk would be better for this definition. Radiological and chemical material inventory (in curies for each radionuclide and grams for each chemical) available to be acted upon by a given physical stress should be evaluated.

The MAR for both technologies is the radioactive liquid waste and toxic material transferred from DST to the RMF or the CFF feed vessel. The quantity of MAR in the CFF system is slightly higher due to the size of the feed vessel and the larger recirculation loop ( $\sim 0-500$ gallons for RMF and several thousand gallons for the CFF). There is no relative differences in the chemical MAR between these filtration technologies; both require periodic chemical flushing using similar chemicals.

The individual evaluations of Quantity of Material at Risk for each of the entrained solids filtration technologies can be found in CFF-1.1.1 and RMF-1.1.1.

\subsubsection{Concentrations of MAR}

Evaluation of the concentration of radiological (unit liter dose) and chemical (unit sum of fractions) MAR is important as it is the amount of material available to be acted on by a given physical stress. Lower concentrations of MAR would be better for this definition.

Radiological MAR concentration was evaluated for the Cs-loaded concentrate being returned to the DST and the Cs-depleted LAW supernatant. A table of radiological MAR concentration for each technology was developed and is provided with its respective assessment form (RM-1.1.2 and CFF-1.1.2). Based on flow sheet data, neither technology affects the concentration of MAR, because the concentration of 
entrained solids has been assumed, rather than quantified. However, if the concentration and composition of the entrained solids were known, it has been assumed that both technologies would have the same affect on the concentration of MAR.

Temporary Emergency Exposure Limits (TEELs) were used for the short-term chemical concentration limits; specifically consideration was given to TEEL-3 values. Both technologies have sum of fractions values for the chemical MAR similar to those for tank farm waste.

The individual evaluations of Concentrations of MAR for each of the entrained solids filtration technologies can be found in CFF-1.1.2 and RMF-1.1.2.

\subsubsection{Dispersability of MAR}

Dispersability of material at risk is lowest for solids and increases from liquids to powders to gases. The dispersible form of MAR needs to be evaluated throughout the process. Less dispersible forms would be better for this definition

The dispersible form of the MAR for both filtration processes is as a liquid with entrained solids. The liquid may become aerosol if components are not properly handled within the system. The concentrate from these processes must be returned below the surface of the waste in the tank to avoid any aerosol problem. In CFF, the return line is maintained below the head space in the DST. However, it is not clear for the RMF whether the return line is maintained below the head space, based on current pre-conceptual design information. Because of this uncertainty, the RMF has a potential of converting the MAR to a dispersible form, i.e., becoming an aerosol under certain circumstances. Engineered features should be implemented to prevent creation of an aerosol.

The individual evaluations for MAR dispersion for each of the entrained solids filtration technologies can be found in CFF-1.1.3 and RMF-1.1.3.

\subsubsection{Dispersive Energy}

For dispersive energy inherent in process parameters (e.g., heat, off-gas, pressure), internal processinitiated events were looked at for these two filtration technologies. For the RMF system, rotational kinetic energy is supplied by the action of the rotating filter plates in the micro-filter housing and waste feed flow. Dispersive energy is minimal due to the construction of the rotary micro-filter unit and relatively low flow. The system pressure is similar to routine tank waste transfers and the rotary disks rotate above $1,000 \mathrm{rpm}$ but the disks have only a 5-inch radius. Therefore, dispersive energy of the housing is limited to leaks and ruptures resulting in a spray or spill hazards. The CFF uses very high flow (approximately 1,100 to $3,300 \mathrm{gpm}$ ) to circulate waste supernate from the filter feed vessel through the cross-flow filter unit. For both filtration systems, the system pressure is similar to routine tank transfers.

Release of dispersive energy would occur as the result of failure of a filtration component or an operational upset condition. The RMF system has significantly lower flow rates then CFF. However, the rotational energy of the spinning disks will have to be evaluated as part of a housing failure accident. Failure of the cross-flow filter units is evaluated as unlikely during the IPS 5-year mission, based on the fact the filter units are passive. Since the supernate is provided at high supply flow rate with a high capacity pump, the dispersion energy would be significant in the event of a component failure.

The individual evaluations of Dispersive Energy for each of the entrained solids filtration technologies can be found in CFF-1.1.4 and RMF-1.1.4. 


\subsubsection{Process Stability}

Assessment of this definition includes qualitative evaluation of the inherent process stability, e.g., the proposed control system, method of shutdown, and ease of shutdown. Many of the shutdown and startup features are not defined yet. Easier and faster shutdown is better as it improves quickness of response in an emergency condition.

The CFF and RMF processes operate under pressurized conditions ( 40 psi for the filtration) and at normal tank temperature of approximately $40^{\circ} \mathrm{C}$. In both cases, rapid shutdown is accomplished by shutting off the feed and the recirculation pumps. RMF filter motors would also be shutdown. This can be designed to be achieved in an easy manner. For a long term shut down both systems will require appropriate flushing of the filter housing/recirculation loop and tank. The CFF is perceived to be an easier system to control with a single feed pump and one filter train. The RMF system has from 4 to 12 filter trains each with its own feed pump.

The individual evaluations of Process Stability for each of the entrained solids filtration technologies can be found in CFF-1.1.5 and RMF-1.1.5.

\subsubsection{Tank Farm Hazard}

The selected process must not create any new (or minimal) tank farm (TF) operation hazard or exacerbate an existing tank farm hazard. Based on the Tank Farm Documented Safety Analysis (DSA) defined accident scenarios for tank farm hazard evaluation, potential hazardous conditions were collected into candidate accident groups sharing similar accident phenomenology for this assessment. Both filtration technologies were evaluated in a qualitative manner for their potentials in creating hazards specifically to the TF operation only. Their potential TF impacts are shown below in Table 3-1.

Table 3-1 - Potential Tank Farm Hazards from Filtration Technologies

\begin{tabular}{|l|c|c|}
\hline \multirow{2}{*}{$\begin{array}{c}\text { Tank Farms Representative Accident } \\
\text { Scenarios }\end{array}$} & \multicolumn{2}{c|}{ Potential to impacts on Accident/Hazard } \\
\cline { 2 - 3 } & Cross-flow Filtration & Rotary Micro-filtration \\
\hline Flammable Gas Accidents & No & No \\
\hline Nuclear Criticality & No & No \\
\hline Vacuum Exhaust Line Rupture & No & No \\
\hline Release from Contaminated Facility & No & No \\
\hline Tank Failure Due to Excessive Loads & No & No \\
\hline Above Ground Structure Failure & No & Yes \\
\hline Mixing of Incompatible Materials & Yes & No \\
\hline Waste Transfer Leak & No & No \\
\hline Unplanned Excavation / Drilling & No & \\
\hline
\end{tabular}

Both filtration technologies have potentials for generating additional TF hazards only due to one accident scenario: mixing of incompatible materials. For other remaining eight scenarios, they do not present any additional $\mathrm{TF}$ operation hazard.

Mixing of incompatible materials Periodic chemical cleaning of the cross-flow filters and rotary microfilter assembly will be required to support longer operating life. Baseline chemical cleaning will be performed using primarily a $0.1 \underline{\mathrm{M}} \mathrm{NaOH}$ solution once per batch. A less frequent $2 \underline{\mathrm{M}} \mathrm{HNO}_{3}$ cleaning is anticipated as well. The current TF DSA prohibits bulk chemical additions to the Tank Farms (e.g., acids). Because it is mounted directly on the DST feed tank, the potential for mixing of incompatible 
materials becomes more of an issue for the RMF system. It will be more difficult to design and install a system that would collect and neutralize the cleaning acid prior to its return to the DST. However, alternative cleaning materials that would be compatible with the DST feed tank, e.g., oxalic acid, could potentially be used in place of nitric acid. In both cases, proper safeguards must be installed to assure full compliance with TF DSA. This may include, but not limited to, alternative cleaning materials, $\mathrm{pH}$ adjustment, corrosion inhibitor adjustment, sampling and verification, etc. prior to transfer to DST.

The individual evaluations of Tank Farm Hazard for each of the entrained solids filtration technologies can be found in CFF-1.1.6 and RMF-1.1.6.

\subsubsection{Fire Hazard}

A system and/or facility containing materials with little or no fire hazard are more acceptable from the fire safety point of view. Fire hazard concerns include consideration of combustible or explosive material, production of flammable gas or by-products, high-heat system, etc. A qualitative evaluation of combustible loading inherent to each filtration technology was performed for this definition.

Cross-flow filter units are passive and its feed pumps are located within the IPS facility. The CFF and RMF systems will have to be evaluated for flammable gas retention and release due to solids in the system during shutdown and start up. The solids hold up volume is larger in the CFF system.

The individual evaluations of Fire Hazard for each of the entrained solids filtration technologies can be found in CFF-1.1.7 and RMF-1.1.7.

\subsubsection{Reactive Chemicals}

Neither filtration technology uses any chemicals during processing. Flushes and filter cleanings are achieved by using water, caustic solution and/or nitric acid. WTP also has included nitric acid cleaning in the base line CFF process for its Pretreatment facility. Because concentrations of these solutions are low, no reactivity issues are anticipated. The issue of whether the DST system can accept nitric acid cleaning solutions from the RMF needs to be further evaluated. If used, nitric acid cleaning will require controls and further evaluation and changes to the TF DSA before implementing its use (see Section 3.1.1.6).

The individual evaluations of Reactive Chemicals for each of the entrained solids filtration technologies can be found in CFF-1.1.8 and RMF-1.1.8.

\subsubsection{Criticality Safety}

From the nuclear criticality point of view, any process that is inherently sub-critical is preferred over a process that may have some potential to overcome sub-critical condition and therefore would require criticality controls to maintain and monitor its sub-critical level. The IPS feed vector contains minimal amounts of transuranic material as shown by the staging feed composition. The IPS assessment looked at two major factors. These are: (1) Does the process have less than the minimal critical mass, and (2) Does the process alter the form and/or distribution of the TF waste.

Criticality under the existing tank farm conditions has been determined to be not credible, because the fissile material concentration is too low and the neutron absorbers are too abundant. The entrained solids that are removed in the CFF and RMF under processing conditions are not changed from the conditions in TF. In addition, after the entrained solids are removed the remaining liquid will not be changed from the criticality point of view. 
Table 3-2 -Total Pu-239 Estimation for IPS Feed Vector

\begin{tabular}{|c|c|}
\hline Tank & $\begin{array}{c}\text { Total Pu-239 in Feed Vector, } \\
\text { grams }\end{array}$ \\
\hline AN-104 & 6 \\
\hline AP-101 & 170 \\
\hline AP-102 & 25 \\
\hline AP-103 & 100 \\
\hline AP-104 & 53 \\
\hline AP-105 & 22 \\
\hline AP-107 & 400 \\
\hline AP-108 & 28 \\
\hline
\end{tabular}

The fissile material hold up in the CFF and RMF based on supernatant concentration is well below 15 grams (fissile exempt) per grams of ${ }^{239} \mathrm{Pu}$ in each feed batch of $>1 \mathrm{M}$ gallons. Total waste volume hold up in the RMF unit is not anticipated to be more than a few hundred gallons (with a large safety margin) and more than a couple of thousand gallons in the CFF unit. Based on those concentrations, both processes are sub critical under processing conditions with the projected feed vector. A review of BBI data for the source tanks also shows that even if sludge was transferred the above statement is still true. CFF and RMF do not change the result of the TF DSA evaluation.

The individual evaluations of Criticality Safety for each of the entrained solids filtration technologies can be found in CFF-1.2.1 and RMF-1.2.1.

\subsubsection{Industrial Safety and Hygiene}

Each technology drives facility layout by its equipment and operation and maintenance requirements. Technology should drive to achieve fewer, or less severe, industrial safety related hazards. These hazards include chemicals, noise, hot open surfaces, rotating equipment, access difficulties, elevated working surfaces, industrial sampling, etc. For this assessment of filtration technologies, preliminary facility layouts developed for the processes were used.

The RMS equipment will be housed in a module that is inserted into an existing riser on the DST thus allowing access via entry at the tank farm. This system does not use any extreme hazardous chemicals, but has rotating blades (with adjustable speeds) in the filter housing. This noise may not be of a serious concern since it will be designed appropriately and will not have personnel nearby for routine operation. If used in conjunction with the FC process, RMF modules will be housed within the IPS facility. In that case, RMS equipment may be located below-grade in a radiation zone and confined space. Remote maintenance access will be required to minimize industrial safety and hygiene concerns.

The CFF equipment will be located within the IPS facility and is shown to be located in the below grade area for all three cesium separation technology. Thus access and egress is only from above and would be a confined space entry into a radiation zone for any maintenance or replacement activities. The CFF system does not use any extreme hazardous chemicals and has no elevated temperature surfaces or rotating components creating noise hazards. The CFF recirculation pump is of much higher capacity and will have some noise associated with its continuous operation.

The individual evaluations of Industrial Safety and Hygiene for each of the entrained solids filtration technologies can be found in CFF-1.3.1 and RMF-1.3.1. 


\subsection{REGULATORY/STAKEHOLDER ACCEPTANCE}

\subsubsection{Achieve Tribal Nations/Stakeholder Acceptance}

From Tribal Nations/stakeholder acceptance point of view, the earlier a technology can be implemented the better it is. They are not necessarily interested in waiting for a perfect system to be implemented in the future, if the technology can remove or reduce the environmental risk as soon as possible. They want to know the extent of land that potentially would become contaminated ground. If a technology can achieve early treatment of waste with a minimum use of new land that can be contaminated, that technology will have a higher probability of being acceptable to the stakeholders.

With respect of achieving early pretreatment both filtration technologies were judged to be capable of implementation in order to make early pretreatment viable, based on available technology maturation assessment information. CFF technology does not require special design and development and industry standards are adequate for implementation. Even though RMF technology requires some re-design and development of a module for application on Hanford DSTs with the 42-inch diameter riser, it is assessed that the redesign work is not significant and can be accommodated to support implementation schedule, if selected.

The RMF system is installed in a DST riser and does not involve a special land use need. If FC is selected for cesium separation, a larger RMF will be co-located with the FC equipment and will require an additional $500 \mathrm{ft}^{2}$ of land use. For the CFF system, a land use of $400-600 \mathrm{ft}^{2}$ is estimated. This land is assumed to be part of the facility land use identified for cesium separation technologies.

The individual evaluations of Achieve Tribal Nations/Stakeholder Acceptance for each of the entrained solids filtration technologies can be found in CFF-2.1.1, CFF-2.1.2, RMF-2.1.1, and RMF-2.1.2.

\subsubsection{Achieve Regulator Acceptance}

From a regulatory perspective, the selected technology must be in compliance with all applicable regulations such as RCRA, CAA, NESHAPS, NEPA/SEPA, and DOE Orders, in order for it to be acceptable. The regulators also want to ensure that disposal system performance is maintained or improved, that secondary waste generation is minimized, and that it can be safely disposed. Any impact to other permitted facilities must also be looked at to ensure that no adverse impacts are found for those facilities.

It is important to understand whether the selected technology will meet current requirements and can be permitted. For National Environment Policy Act (NEPA), it is assumed that early LAW pretreatment with either of these filtration technologies will be covered by the Tank Closure and Waste Management (TC\&WM) Environmental Impact Statement (EIS) (expected completion in December 2009) and issuance of its Record of Decision (ROD) that is expected in January 2010. Three notices of construction will be required for the IPS and temporary storage tanks. The IPS and temporary tank storage units will constitute Resource Conservation and Recovery Act (RCRA) treatment, storage, and disposal (TSD) units and will require submittal of a Part B Permit Application pursuant to WAC 173-303-806, and Ecology issuance of a final status RCRA Part B permit prior to operation (WAC 173-303-840).

Neither of these technologies has been previously permitted for use, but basic information exists to support the permit application process. It is anticipated to take 32 months for both technologies. No unusual difficulties are anticipated in obtaining various regulatory and stakeholder acceptance based on existing regulatory processes and requirements. RCRA, NEPA, SEPA, and CAA/NESHAPS requirements can be satisfied. 
State regulators have been amenable, in the past, to expediting the permit process to accelerate cleanup (e.g., demonstration bulk vitrification program and IDF). Unless the NEPA evaluation determined that one of the filtration technology required an Environmental Assessment (EA), while none of the others did, regulatory compliance does not appear to be a discriminator. For this assessment, one has to look at the total IPS facility as these permits apply to the total facility.

The cross-flow filtration process and rotary micro-filtration systems do not produce any new secondary waste forms requiring disposal. No secondary waste requiring Effluent Treatment Facility (ETF) processing is generated as a result of RMF or CFF activities. Items such as personnel protection equipment (PPE), failed equipment, etc. can be disposed of during Tank Farms operations. Quantity of failed equipment requiring disposal is undetermined at this time for both technologies. Constituents of potential concern (COPC) issues are not applicable to either of these technologies.

The clarified LAW supernatant is transferred to the cesium separation process while the solid concentrate is returned to an adjacent DST. It is also assumed that the caustic and acid solutions used to periodically clean the filters will either be routed with the LAW supernatant to the cesium separation process or with the solid concentrate back to the DST.

The individual evaluations of Achieve Regulator Acceptance for each of the entrained solids filtration technologies can be found in CFF-2.2.1 through CFF-2.2.4 and RMF-2.2.1 through RMF-2.2.4.

\subsection{TECHNICAL MATURITY/FLEXIBILITY}

Technical maturity and flexibility of the selected technology is a crucial factor in the successful completion of the mission. Mature technology provides sufficient information for the design of the process equipment, services and facility. Technical maturity of a technology is measured by assessing a Technology Readiness Level (TRL) of a technology. Detail description and methods to establish a TRL for a given technology for DOE projects is described in the DOE Process Guide, DOE 2008, Technology Readiness Assessment (TRA) / Technology Maturation Plan (TMP) Process Guide, US DOE Office of Environmental Management, March 2008. In this process, a technology can receive a TRL of 0 to 9 , where 9 represent the most matured technology. Once a TRL is determined for a technology, each project must try to mature the process by attaining the highest TRL for the selected technology. Efforts (resources and time) required to mature the process would be different for each technology and should be determined. Along with these efforts, knowledge about the probability of success in attaining the fully matured technology is very useful. Therefore in this section along with the TRL, an estimate of effort to mature each technology and its probability of success for each technology under consideration are developed.

For Hanford waste treatment projects, a flexible technology will provide a large operation boundary so that it can be successfully applied to a variety of tank waste with differing characteristics and properties. The IPS technology flexibility is measured with factors such as ability to process variety of feeds, ability to adjust process rate, flexibility to modify product, expandability, capability to recover from out of specification product, and applicability to other DOE complex projects.

Senior level management personnel conducted a TRL workshop (May 13-14, Richland, Washington) to review and assess technical maturity and process flexibility and robustness of each filtration technology under consideration for application for the IPS. This workshop conducted this review based on the guidance provided in the DOE Process Guide (DOE 2008). This TRL workshop also used the data developed for earlier technology readiness assessment for the supplemental treatment program, DOE/ORP-2007-01, Technology Readiness Assessment for the Supplemental Treatment Program, October 
2007. In this prior 2007 workshop, technology readiness assessment was performed for both filtration technologies. Responses developed at that time were re-looked at and adjusted as needed for the current status of each technology.

In the following sections, technical readiness level (Section 3.3.1) and Process flexibility and robustness (Section 3.3.2) of filtration technologies are summarized.

\subsubsection{Technology Readiness Level}

\subsubsection{TRL Number}

An expert panel reviewed the technology readiness level 4 summaries for the RMS and CFF systems from earlier study (DOE/ORP-2007-01, Technology Readiness Assessment for the Supplemental Treatment Program). Between 2007 and 2008, no major technology readiness level changes were found to modify their individual TRL of 3 assigned in 2007 study. Since 2007 , conceptual designs are being developed for both technologies but cannot be credited as this design report is not fully documented. Key physical and chemical properties have been characterized for a range of wastes using simulants developed based upon waste data for AN-105 (Envelop A) and AN-107 (Envelop C) wastes. Limited safety issues are being examined for down select, but no control strategies and/or limits are being explored.

Some of the TRL qualifying level 4 activities not performed so far include: modeling and simulation to define interfaces between components, overall system requirements for end user's application, laboratory testing requirements derived from established system requirements, science and technology exit criteria. A detailed list of incomplete items can be found in respective assessment forms RMF-3.1.1 and CFF3.1.1. Even though the 2008 review added four more criteria for level 4 , both technologies still have 10 unsatisfied criteria out of 34. Due to such large number of unsatisfied criteria, both technologies maintain their individual TRL number of 3.

The individual evaluations of TRL Number for each of the entrained solids filtration technologies can be found in CFF-3.1.1 and RMF-3.1.1.

\subsubsection{Effort to Mature Technology (Cost and Schedule)}

A rough order-of-magnitude (ROM) cost and schedule was estimated for developing the candidate solids separation technologies to TRL Level 6, allowing transition into final design (consistent with DOE/ORP2007-01). The effort covered testing for technology maturity only (i.e., development and qualification testing). Not include was factory acceptance, construction acceptance, or operational testing costs; these three project testing activities and all other project costs are covered by other assessments. This is a ROM estimate for comparison purposes only and final estimates will be documented in the Technical Maturation Plan. Table 3-3 presents the results of this effort.

Table 3-3 - Effort to Mature Solids Separation Technologies Comparison

\begin{tabular}{|c|c|c|}
\hline Technology & $\begin{array}{c}\text { Rough order-of-magnitude cost } \\
\text { (million dollars) }\end{array}$ & $\begin{array}{c}\text { Rough order-of-magnitude } \\
\text { schedule (months) }\end{array}$ \\
\hline Rotary Micro-filtration & 2.0 & 30 \\
\hline Cross-flow Filtration & 2.5 & 36 \\
\hline
\end{tabular}

The individual evaluations of Effort to Mature Technology for each of the entrained solids filtration technologies can be found in CFF-3.1.2 and RMF-3.1.2. 


\subsubsection{Probability of Success}

A subjective analysis of risk associated with the candidate solids separation technologies was performed. Major risks were identified and evaluated for impacts to cost and schedule, resulting in the following classic ranking:

High Probability of Success: Worst case risk is Medium Medium Probability of Success: Worst case risk is High Low Probability of Success: Worst case risk is Very High

Based on the analysis, both RMF and CFF were judged to entail a high probability of success. The individual evaluations of Probability of Success for each of the entrained solids filtration technologies can be found in CFF-3.1.3 and RMF-3.1.3.

\subsubsection{Process Flexibility and Robustness}

\subsubsection{Ability to Process Variety of Feeds}

From overall Hanford mission perspective, it is always advantageous to select a technology that is flexible to expand its application to various tank farm waste feed material, even though they are not currently being considered for the IPS project. This capability represents technology flexibility to process a variety of Hanford feeds.

This is a subjective evaluation based upon existing configuration information, past test results, and understanding of unit operations. This effort supports determination of the overall Technology Readiness Level. Critical input stream characteristics include:

- Solids concentration

- Solids type/structure (e.g., round or elongated crystals, flocculent or sludge)

- Solids size

- Summary information is organized by critical input stream characteristics for each of the filtration technologies.

SRNL testing showed that RMF flux rates, using simulated SR tank wastes, ranged between 0.12 and $0.29 \mathrm{gpm} / \mathrm{ft}^{2}$ for entrained solids concentrations of 0.06 to $15 \mathrm{wt} \%$, using simulated SR tank wastes. (WSRC 2006). Based on limited testing, RMF appears slightly sensitive to particle size, shape and structure, since the filter media pores and solids transport area may become plugged depending upon particle crystal type and size. Chemical cleaning systems appear to be successful in resolubilizing or breaking up sludge mass. Chemical cleaning is planned for IPS design, but requires additional design to ensure minimal impacts to the tank if the system is located in a tank riser. The installation of air back pulsing and use of low-shear pumps will also be included in the IPS design to avoid reducing solids particle size and/or to minimize particle uniformity.

ORNL testing showed that CFF flux rates, using simulated SR tank wastes, ranged betweem 0.02 and $0.14 \mathrm{gpm} / \mathrm{ft}^{2}$ for entrained solids concentrations of 0.1 to $15 \mathrm{wt} \%$ (ORNL 2001). ORNL testing also qualified controlling factors for filter flux depending upon solid concentrations, e.g., in the Mott filter flux was controlled by transmembrane filter drop for concentrations $<5 \mathrm{wt} \%$ and by axial velocity for solids at $10-15 \mathrm{wt} \%$. Savannah River testing of AN-104 tank waste measured an average filter flux rate of $0.085 \mathrm{gpm} / \mathrm{ft}^{2}$ particle sizes of $0.8-1.7$ micron (WSRC 2003).

CFF is sensitive to particle shape only at small particle sizes; spherical particles tend to clog the filter pores. Chemical cleaning systems are successful in resolubilizing or breaking up sludge mass and will be 
included in the IPS design. The installation of air back pulsing and use of low-shear pumps will also be included in the IPS design to avoid reducing solids particle size and/or to minimize particle uniformity.

The individual evaluations of Ability to Process Variety of Feeds for each of the entrained solids filtration technologies can be found in CFF-3.2.1 and RMF-3.2.1.

\subsubsection{Ability to Adjust Process Rate}

A subjective evaluation was conducted on the ability of candidate solids separation technologies to adjust the input feed rate while maintaining a stable operation. This evaluation was based upon existing configuration information, past test results, and understanding of the unit operations. The technology was categorized as follows:

- High Rate Flexibility = Can adjust process feed rate across wide range while having minimal effect on operation,

- Medium Rate Flexibility = Can only slightly adjust feed rates while maintaining stable operation,

- Low Rate Flexibility $=$ Slight feed rate adjustment will have high probability of process upsets and require significant effort to recover technology stability.

The ability to adjust the process rate for the RMF system is judged as being "High" because of two technical bases identified through testing (WSRC 2006). First, the nature of RMF testing has established a degree of freedom between the pressure and axial velocity flow similar to CFF.

"The SpinTek ${ }^{\mathrm{TM}}$ rotary filter is a compact filtration system that uses membrane filters mounted on rotating disks. The flux advantage of the rotary microfilter compared to other membrane processes results from the high shear and centrifugal force acting on the boundary layer next to the membrane. This shear greatly reduces fouling of the membrane surface and increases fluid flow through the membrane. Pressure is decoupled from the feed flow rate, allowing more control over the driving force pressure and independent control of the shear applied to the filter cake." (WSRC 2006 page 3)

Similar flexibility for independent control of membrane shear is provided by the RMF technology. While employing a fixed shear-inducing blade, cleaning of solids is also impacted by varying flow rates. However, more applicable is the adjustment potential for varied disk rotation rates, providing similar, if not slightly better, potential for solids buildup compensation with varying feed rates.

The ability to adjust the process rate for the CFF system is judged as being "High" because of two technical bases identified through testing (BNF 2000). First, the nature of CFF testing establishes a degree of freedom between the pressure and axial velocity flow.

"A cross-flow filter (XF) is significantly different from a dead-end filter in that the main slurry flow is not forced through the filter medium. Instead, the slurry flows parallel to the filter substrate while allowing the filtrate to be removed perpendicularly, as a result of the transmembrane pressure (TMP). In this way the XF is basically self cleaning as the turbulent slurry flow tend to shear solids away from the filter wall as they try to adhere. However, because of this cross flowing stream, there is an added degree of freedom. For instance, an increase in slurry velocity may, but not necessarily, lead to an increase in filtrate flux.

This is because the slurry system pressure can be made to decrease. That is, the slurry system pressure can be controlled independently from the slurry axial velocity and both of these quantities will affect the rate of filtrate flow." (BNF 2000 page 22) 
Both parameters affect perpendicular transport of the filtrate across the transmembrane. It is possible to vary filter pressures in addition to changing flow rates to affect the filter flux. This provides significant flexibility of process operations with varied feed rates.

Secondly, changing feed rates affect the shearing of solids thus providing a self-cleaning value to varying flow rates. It is possible to adjust feed flow rates to compensate for solids buildup in the filter membrane pores.

As a result, both RMF and CFF were deemed to exhibit High Process Rate Flexibility. The individual evaluations of Ability to Adjust Process Rate for each of the entrained solids filtration technologies can be found in CFF-3.2.2 and RMF-3.2.2.

\subsubsection{Flexibility to Modify Product}

A subjective evaluation was conducted on the candidate solids separation technology's process flexibility and robustness. The following binning is arbitrarily defined to establish flexibility based upon degrees of freedom of technology choices and process control parameters.

- High product flexibility $=>12$ degrees of freedom.

- Medium product flexibility $=<12$ and $>5$ degrees of freedom

- Low product flexibility $=<5$ degree of freedom

This evaluation judged RMF and CFF to have medium filtration product flexibility. The individual evaluations of Flexibility to Modify Product for each of the entrained solids filtration technologies can be found in CFF-3.2.3 and RMF-3.2.3.

\subsubsection{Ability to Expand}

This is a subjective evaluation of current capability based upon existing configuration information, past test results, and understanding of the unit operations. This evaluation supports determination of the overall Technology Readiness Level and assumes that the ability to expand is based upon additional volume/mass only with all other waste conditions remaining constant. Critical expansion characteristics include:

- Ability of existing design to handle additional feed volumes

- Ability for installation of addition separation unit operations into existing footprint

The CFF technology is sized to meet required filtrate rates with little or no extra capacity for any of the Cs separation technologies, based upon predicted filtration rates (CH2M HILL 2008):

Excess Capacity: IX-sRF $=0 \% ; \mathrm{FC}=11 \%$; and $\mathrm{CSSX}=2 \%$

The pre-conceptual IPS layout has additional capacity for additional cross-flow filters in a horizontal configuration or if system was realigned to vertical configuration. The footprint has the capability to be easily modified for additional filters if needed.

The RMF technology is sized to meet required filtrate rates with extra capacity for IX-sRF and CSSX, but no additional capacity for FC based upon predicted filtration rates (CH2M HILL 2008):

Excess Capacity: IX-sRF $=41 \% ; \mathrm{FC}=0 \%$; and $\mathrm{CSSX}=45 \%$ 
The pre-conceptual IPS layout has no additional capacity for additional rotary micro-filters in tank AP104, without significant redesign (more platters or more than two units per riser). This would require installation of a much larger vault area for the IX-sRF and CSSX options than is currently designed for the $\mathrm{CFF}$ footprint. The FC footprint would require a much larger footprint for any volume increase, since there is currently no excess capacity.

This evaluation judged RMF and CFF to have medium filtration product flexibility. The individual evaluations of Ability to Expand for each of the entrained solids filtration technologies can be found in CFF-3.2.4 and RMF-3.2.4.

\subsubsection{Recover from Out-of-Specification Product}

Each process system was assessed for flexibility to recover and reprocess the product that does not meet specification requirements. The out of specification product may hinder the facility operation severely and could lead the facility shutdown until the problem is resolved. Therefore, this is an important measure to assure that the selected process can accommodate this need when required.

This is a subjective analysis based upon the current process flow sheet, without specific systems assumed for recycle and storage to address out of specification product. The flexibility is defined based on required additional equipment cost to recover from such a condition. These flexibility groups are defined as follows:

Highly flexible: System that allows product recycling without additional hardware systems.

Medium flexible: $\quad$ System requiring minimal storage or processing or software modification to recover (e.g., $\$ 1 \mathrm{M}-\$ 5 \mathrm{M}$ )

Low flexibility: System requiring significant storage or processing or software modification to recover (e.g., $\$ 5 \mathrm{M}-\$ 20 \mathrm{M})$

Very Low flexibility: Final product can no longer be further processed to improve specification compliance and must be disposed of as out-of-specification product.

Because no additional RMF units can be installed on the DST riser, any out of specification product may necessarily to be returned to the DST tank. It may require either replacement of filter modules or require installation of extensive piping and valving, and potentially other tank and pump. The cost of such additions could be $>\$ 15 \mathrm{M}$. At this time, no specific product requirements have been identified. Based on the cost it is rated as having "Low" recovery flexibility.

The CFF process flow sheet includes a feed receipt tank for collection of filtered product. A recycle line can be installed from that tank to allow refiltration of product material within the IPS using existing tanks and pumps. A rough order of magnitude estimate is less than $\$ 5 \mathrm{M}$. Therefore, the CFF is rated as having a "Medium" recover flexibility.

The individual evaluations of Recover from Out-of-Specification Product for each of the entrained solids filtration technologies can be found in CFF-3.2.5 and RMF-3.2.5.

\subsubsection{Technology Applicability to Other DOE Complex Projects}

In a subjective analysis, an attempt was made to define filtration technology interface in relationship to other pretreatment activities occurring across the DOE complex. Applicability standings for these subjective assessments are defined as follows: 
High applicability: Identical technology was or is being deployed in other DOE site(s) or projects for full-scale operation.

Medium applicability: Identical technology is under investigation at other DOE site(s) for application.

Low applicability: Identical technology is planned for investigation at other DOE site(s) for application.

No applicability: $\quad$ No further planning of deploying this technology is being considered at any other DOE complex site or project.

Based on above applicability definitions, the RMF's technical applicability is rated "Medium". This technology is being investigated (design, fabricate, and perform testing on a full-scale system) for potential SRS tank farm applications. It is not deployed nor planned for final deployment in full-scale hot operations at this time.

Similarly, based on above applicability definitions, the CFF technology's technical applicability is rated "High". This technology is being deployed by the Hanford WTP. This technology has also been successfully deployed at WVDP, SRS, and ORNL.

The individual evaluations of Technology Applicability to Other DOE Complex Projects for each of the entrained solids filtration technologies can be found in CFF-3.2.6 and RMF-3.2.6.

\subsection{OPERABILITY AND MAINTAINABILITY}

IPS facility requires significant operation and maintenance support. These activities play an important role in the success of the mission. To balance the technology selection, various operation and maintenance related measure were evaluated. These measures include ease of process control and operation, ALARA principles, reliability of process equipment, ease and frequency of maintenance, ease of process implementation, and liquid/solid secondary waste. Both filtration processes were evaluated against these measures and assessments are provided below.

\subsubsection{Ease of Process Control and Operation}

Many factors affect ease of process control and operation. For radioactive operation, easier process control and operation is considered to result in the effective operation with a minimum of errors and accidents. Items such as system complexity, number of chemicals, process samplings needs, operating margin and flexibility, standby conditions, etc. are various factors that measure "ease of process control and operation" and must be reviewed. At Hanford, where interfaces with the tank farms are very crucial, complexity of transfers to, from, and within Tank Farms is also important.

\subsubsection{Minimize Number and Frequency of Surveillances}

Various process parameters measurements and recordings as well as leak detection are required to properly monitor operations of this kind of radioactive processes. Types of process parameters include tank levels, pump amperage, temperature, pressure, etc. Preliminary review of RMF operations identified a minimum of 35 process parameters that require measurement and recording during routine $\mathrm{RMF}$ operation for the IX-sRF and CSSX systems. This is based on two waste feed pumps and four RMF units for these two cesium separation technologies. If RMF is used with the FC system, the number of filter 
units required will be twelve and the total process parameters requiring measurement and recording will be at least seventy one (71).

For the CFF system operations, at least twenty two (22) process parameters have been identified requiring measurement and recording during routine operation. The total list of these process parameters is provided with each respective assessment form. These parameters include item, such as, waste feed pump parameters, filter motor parameters, feed tank level, etc. Many of these system-monitoring parameters are comparable to current waste transfer operations and process operations similar to the waste retrieval from the 200 series tanks. Detailed design may identify more parameters for measurements.

The individual evaluations of Minimize Number and Frequency of Surveillances for each of the entrained solids filtration technologies can be found in CFF-4.1.1 and RMF-4.1.1.

\subsubsection{Minimize Number of People to Operate}

A preliminary assessment shows about an equal number of operating personnel ( 5 to 6 ) will be required for each filtration process. The combination of RMF with CSSX and IX-sRF will require a minimum of five (5) people for routine operation of filtration system. For the CFF system with all three cesium separation technologies and RMF-FC system combination, at least six (6) operation personnel will be required.

The individual evaluations of Minimize Number of People to Operate for each of the entrained solids filtration technologies can be found in CFF-4.1.2 and RMF-4.1.2.

\subsubsection{Ease of Startup and Shutdown}

The CFF system has only two moving components, which are waste transfer pump and the waste feed pump. Hanford workers are experienced in such equipment operation. The CFF system is easy to start up and shutdown. Some activities such as water flushing of the filter system may require an extended shutdown of more than five (5) days. Temporary or emergency shutdowns will require only stopping of these two (2) pumps and can be accommodated with a proper equipment design.

The RMF system has a total of six moving components (two waste pumps \{transfer and feed and four RMF units) when coupled with CSSX and IX sRF ion exchange systems. When used with the FC system, the RMF system has a total of twelve (12) RMF units. In such case, startup work is required in two different locations, valve line-ups in the tank farm and filter modules in the IPS facility. Such an operation is a bit complex due to administrative control procedures, such as, lock removal, communications, safety reviews, independent reviews, etc.

The individual evaluations of Ease of Startup and Shutdown for each of the entrained solids filtration technologies can be found in CFF-4.1.3 and RMF-4.1.3.

\subsubsection{Minimize System Complexity}

The system complexity is ascertained by assessing the number of parts and transfer routes. The RMF system coupled with CSSX and IX-sRF processes has six (6) components as described earlier and two waste transfer lines external to DST housing. The RMF pump capacity is smaller compare to CFF system pump. This seems to be the average or less than average for system complexity. For use with FC the system, the RMF system also has two additional transfer lines and higher number of active components as described earlier. The higher number of components does increase the complexity of the RMF system when used with the FC process. 
The CFF system includes a waste transfer pump, waste feed pump, filter, air blowback system and four (4) primary transfer lines (line from waste transfer pump to feed storage tank, line from feed tank to filter, filtrate line to storage tank, and solids return line). The pumping systems are no more complex than any of the existing waste transfer systems currently used in the tank farm. The CFF system has only three (3) active components and 4 primary transfer lines. The system has simple unit operations - pumping, passive filtration, liquid transfers and monitoring. It is not considered to be a complex process.

Therefore both filtration systems are not considered to be complex. Both of them can be easily operated from a standby condition. The individual evaluations of Minimize System Complexity for each of the entrained solids filtration technologies can be found in CFF-4.1.4 and RMF-4.1.4.

\subsubsection{Minimize Number of Chemicals Needed}

Both the RMF and CFF system uses two chemicals (sodium hydroxide and nitric acid) in moderate quantities and concentrations for cleaning of the filter media. In the RMF system, supply of chemical solutions for the flushing system in the tank farm may increase operation complexity as the flushing system is not fully designed. For the CFF the supply of sodium hydroxide and nitric acid for filter module cleaning will be supplied from within the IPS facility. This CFF tube bundle cleaning will be performed with in the IPS facility. Use of nitric acid within the IPS facility poses no higher demands from the operation point of view.

The individual evaluations of Minimize Number of Chemicals Needed for each of the entrained solids filtration technologies can be found in CFF-4.1.5 and RMF-4.1.5.

\subsubsection{Process and Regulatory Samples}

Filtration systems (both) have minimum process sampling needs. Two types of samples, the waste feed to the filter and wash solutions prior to transfer to DST are needed. Entrained solids transferred to the DST do not require regular sampling as they are not modified during the filtration process. No regulatory samples are identified at this time.

The individual evaluations of Process and Regulatory Samples for each of the entrained solids filtration technologies can be found in CFF-4.1.6 and RMF-4.1.6.

\subsubsection{Batch versus Continuous Operation}

The CFF system supports continuous operation for each DST campaign provided there is enough lag storage available in the IPS facility for waste feed and filtrate solutions. The RMF system can be operated on a continuous basis for a given DST campaign provided enough filtrate storage capacity is provided within the IPS facility. Batch operation can also be supported, if required.

Both filtration systems, operated as batch or continuous process, do not adversely impact tank transfer. The individual evaluations of Batch versus Continuous Operation for each of the entrained solids filtration technologies can be found in CFF-4.1.7 and RMF-4.1.7.

\subsubsection{Ease of Entry and Exit from Standby}

The CFF system is the easiest to startup and shutdown based upon containing only two active components, the waste transfer pump and waste feed pump. Startups and shutdowns will require removal/installation of administrative locks, valve lineups, and independent verification thereof, etc. in two locations (tank farms and IPS facility). 
The RMF system is also considered to be easy to start up and shutdown based on only six moving components. When used in conjunction with the FC system, the RMF system becomes more complex because it has fourteen moving components at two different locations (DST and IPS facility). Startups (new campaign or from standby condition) will require various activities (such as valve lineups, administrative lock removal, etc.) in the tank farms and the IPS facility. Such activities are considered to be more involved.

The individual evaluations of Ease of Entry and Exit from Standby for each of the entrained solids filtration technologies can be found in CFF-4.1.8 and RMF-4.1.8.

\subsubsection{Wide Operating Margin}

The RMF system has an acceptable operating range for the majority of the tank wastes present in the tank farms. Rotational speed of filters can be easily monitored ( 800 to $2,500 \mathrm{rpm}$ ) and adjusted as required. Both filtration systems are anticipated to be applicable for removal of entrained solids from the majority of the tank waste present in the tank farms.

The individual evaluations of Wide Operating Margin for each of the entrained solids filtration technologies can be found in CFF-4.1.9 and RMF-4.1.9.

\subsubsection{Complexity of Transfers to, from and within Tank Farms}

All DST waste transfers for the CFF and RMF systems are considered to be standard tank farm transfers and require no special equipment or processes. These transfers do not increase any difficulty within the tank farms operations. Transfers of spent cleaning solution will require compliance with DST storage specifications. The addition of nitric acid for cleaning of both filtration system components will require a special TF operations procedure.

The individual evaluations of Complexity of Transfers to, from and within Tank Farms for each of the entrained solids filtration technologies can be found in CFF-4.1.10 and RMF-4.1.10.

\subsubsection{ALARA}

For the operation of IPS facility, ALARA represents the most critical operations principle. The facility design and processes must address ALARA principles for the handling of radioactive components as well as other chemicals which can be hazardous or toxic and harmful to personnel and the environment. For handling of these types of components or chemicals, contact maintenance should be minimized to reduce personnel exposure and risk. In this section, operational impacts from filtration technologies for this definition are assessed. Facility related ALARA issues are anticipated to be addressed during the engineering design phases.

The waste feed and transfer pumps, waste transfer piping and the filter assemblies (tube bundles or rotary micro-filter assemblies) are the primary equipment items that will contain radioactive waste and hazardous chemicals. The chemical cleaning/flushing of filters, prior to any maintenance activities, will use $\mathrm{NaOH}$ and/or nitric acid, which are considered to be hazardous chemicals. The CFF system waste transfer pump is located in the pump pit, and the waste feed pump and filter are located in the IPS facility.

The RMF system components are located on the DST riser when coupled with CSSX and IX-sRF systems. The RMF equipment is located in the tank farms and IPS facility when used with the FC system. Even though CFF and RMF systems will be designed for remote replacement to the maximum extent possible, maintenance activities may expose personnel to radiological and chemical hazards. The 
RMF system will have to be removed from the DST for component replacement when coupled with CSSX and IX-sRF because the pumps and filters are located in a 42" riser of the DST feed tank.

The CFF waste feed pump is located in the DST pump pit and requires special procedures for removal/replacement in the $\mathrm{TF}$ environment. Other $\mathrm{CFF}$ system components in the IPS facility requiring replacement or maintenance activities (or RMF components in the IPS facility with FC system) may be performed remotely or may be performed in a controlled area to reduce exposure by using proper procedures and facility design features.

The individual evaluations of ALARA for each of the entrained solids filtration technologies can be found in CFF-4.2.1 and RMF-4.2.1.

\subsubsection{Reliability}

Reliability of components and systems will play an important role in operation and maintenance of the IPS. Passive systems or systems with a lesser number of active components should provide better overall reliability. Also, reliability of analogous systems used in similar environments may provide a better indication of performance within the IPS. These factors for filtration technologies are discussed below.

Both filtration systems use waste feed and waste transfer pumps, which should be able to operate reliably for five years or more as they mainly transfer supernatant. They should remain serviceable until six to seven million gallons are pumped.

The CFF system has another active component, filter blowback system, which is reliable for at least ten years. On the other hand, The RMF system includes 4 or 12 active filter assemblies depending upon the selected cesium separation technology. The rotary micro-filter assemblies should not fail more than once per 3-5 years. The filter rotational shaft bearing have been noted as the most likely components to fail and alternate bearing with air cooling has been recommended by the manufacturer to expand the operating life to 3-5 years.

Rotary micro-filter unit is a new application of commercial technology in a radioactive environment and has limited data for reliability. The system has been tested for radioactive waste applications at SRS and is being implemented there for routine operations. While there is no experience with shell and tube filter systems at the Hanford Site, there is wide use of shell and tube filters in commercial (but non-radioactive) facilities for similar solids removal operations. Waste transfer pump systems have been extensively used at Hanford and have shown good to outstanding reliability.

CFF waste feed pumps are larger than the RMF waste feed pumps, but their size and capacity should not impact reliability. Reliability of CFF system may be a slight better than the RMF system because there are fewer active components in the CFF system.

The individual evaluations of Reliability for each of the entrained solids filtration technologies can be found in CFF-4.3.1, CFF-4.3.2, RMF-4.3.1, and RMF-4.3.2.

\subsubsection{Ease and Frequency of Maintenance}

Maintenance activities play an important role in the overall success IPS facility operation. Ease and reduced frequency of maintenance helps achieve cost effective operation and improves plant's total operational efficiency. To assess this measure various items such support systems, preventative maintenance requirements, type of maintenance and difficulty of maintenance with complex and specialized equipment are considered. For Hanford application, reduction in active personnel interactions with the DST environment is preferred. 
Both filtration systems require periodic cleaning of solids from the filter membranes. Although there is no operational data are available for frequency of such operation, it is anticipated to be relatively low for both filter systems. The RMF and CFF systems require five (5) operation support services - power, air, water, $0.1 \underline{\mathrm{M} \mathrm{NaOH}}$, and $2 \underline{\mathrm{M}} \mathrm{HNO}_{3}$. Nitric acid, water and sodium hydroxide solutions are used for routine cleaning and backwashing the filter modules.

For both systems, equipment will be located in radioactive work zones and will require routine access to those areas. Rotary micro-filter units represent a newer technology but preventative maintenance activities and frequencies are anticipated to be normal. Both systems require visual inspection for leaks and monitoring of pressure drops across the filters on a routine or frequent basis. Pressure drop readings can be monitored from control rooms but gauges will require routine calibration. Other items (such as inspections and greasing of motor bearing inspections, vibration readings, etc.) are needed at less frequent level. Assuming that preventative maintenance activities and surveillances can be performed remotely with systems (such as distributed control system [DCS] monitoring, in-room cameras, auto-lubricators, etc.) radioactive zone entries will be limited to repair/replacement activities. Based on available data, the RMF will require a radioactive zone entry every two years for mechanical seals and the CFF system will require a radioactive zone entry every five years for filter tube bundles replacements.

For the RMF and FC system combination, these frequencies of radioactive zone entries may be much higher. Due to radiation zone limitations, these preventive maintenance activities will involve an increased level of complexity.

If the RMF system is selected in conjunction with the FC system, the number of required rotary filter units will be twelve (12) rather than four (4). This will increase the total number of preventive maintenance activities.

The rotary micro-filter assembly is a specialized equipment item but is based on commercial technology which is modified for radioactive operations. Similar designs are commercially available. Cross-flow filtration technology is commercially available. The unit to support the IPS will require some adaptations/modifications for radioactive service. With such an adaptation/modification, certain spare parts inventory will have to be maintained to support operation and maintenance.

The RMF feed pumps will be installed in the 42 " riser of DST AP-104 while the rotary micro-filtration units will be installed on the 42" riser. AP-104 is designated as a feed tank. This one time installation of the filtration unit will require "DST entry" when the pumps and filter units are installed. On the other hand, the CFF system waste feed pump will be located in the pump pit shielded from the main dose contribution from the process vessels to reduce the dose consequences for maintenance activities. So both systems will require a "DST entry" for installation and some maintenance activities. For RMF, the design will also provide remote feature to the extent possible for ease of performance of routine maintenance activities such as annual cleaning, replacement of failed component (modular design), etc.

The individual evaluations of Ease and Frequency of Maintenance for each of the entrained solids filtration technologies can be found in CFF-4.4.1 through CFF-4.4.5 and RMF-4.4.1 through RMF-4.4.5.

\subsubsection{Ease of Implementation}

One would prefer, for obvious reasons, that the selected technology be easy to implement. If technology implementation and operations are too complex, it will tend to bring in human errors as well as extensive training and retraining programs that may make it less cost effective. Thus, the two solid separation 
technologies are assessed for ease of training, procedure implementation, and familiarity with similar procedures. All these three factors lead to ease of implementation.

Both filtration technologies are based on commercial technologies and, therefore, are reasonably straight forward to adapt to radioactive operations. These types of equipment have been deployed (some at Hanford) and present no significant complexity for training of personnel for operation and maintenance. Both filtration systems do not pose any complexity in operation and maintenance procedures. Although the RMF system is not currently used at the Hanford Site, DOE sites (mainly SRS) will have some operating experience by the time IPS begins its operation. This should be beneficial to Hanford operations.

The individual evaluations of Ease of Implementation for each of the entrained solids filtration technologies can be found in CFF-4.5.1 through CFF-4.5.3 and RMF-4.5.1 through RMF-4.5.3.

\subsubsection{Liquid/Solid Secondary Waste}

Most processes generate secondary waste. It is important to assure compliance with DOE Order 420.1B and operational impacts associated with hazardous (generated) waste handling are minimized.

DOE Order 420.1B provides the general aspects of safety to be included in DOE facilities, and covers Nuclear and Explosion safety design, Fire Protection, Criticality Safety, Natural Phenomenon Hazard (NPH) Mitigation, and the System Engineer Program. A review of the order finds no features of either filtration technology that would not meet the order requirements and both system designs can easily accommodate requirements.

One of the secondary wastes generated by both filtration technologies is the washing and cleaning solutions. The CFF unit is designed for continuous operation, and it promotes self-cleaning of the filter membranes by sloughing off the filtered solids, which are returned to DSTs. The RMF unit is also designed for continuous operation, and the spinning action of the filter pack promotes self-cleaning of the filter membranes by sloughing off the filtered solids which are returned to DSTs. No impact to process operations is anticipated during normal operation. Upon failure of each unit, operations will be required to be suspended. For CFF, this is anticipated to be every 5 years for replacement of filter tube bundles and every 2 years for the RMF mechanical seal replacement. These maintenance activities will generate secondary waste comprised of failed equipment and components.

The individual evaluations of Liquid/Solid Secondary Waste for each of the entrained solids filtration technologies can be found in CFF-4.6.1, CFF-4.6.2, RMF-4.6.1, and RMF-4.6.2.

\subsection{PROGRAMMATIC ASPECTS}

Several programmatic considerations have the potential to impact the selection of the technologies required for the IPS. In some cases, these factors may become predominant and require a more complete and careful evaluation. From a programmatic perspective, the major factors for the IPS technology evaluations are: cost, implementation schedule, DST space management, impacts to other site facilities, and availability of needed resources and material. In the following sections, these factors are assessed for the two filtration technologies, CFF and RMF.

\subsubsection{Cost Impacts}

Tank Farms waste is required to undergo both entrained solids filtration and cesium separation prior to acceptance at the WTP LAW facility. Since there is always a pairing of a solids filtration with a cesium 
separation process, the one with the higher required throughput drives the capacity of the other, and hence the capital and consumables costs. Estimation of the cost of a technology independent of its specific pairing is not practical due to the capacity dependence. Consequently, six separate cost estimates have been developed for the six possible pairings between the two solids separation technologies (RMF and CFF) with the three cesium extraction technologies (IX-sRF, CSSX, FC).

For the purpose of technology selection, costs for ancillary items such as control trailer, transformer, switchgear, motor control center, power transmission lines, water supply lines, and sanitary waste removal have not been included as they have been assumed similar for each option. The capital costs include engineering design, project management, construction management, and the construction costs. The construction costs are based on the quantities developed from the process layouts and equipment list. The life cycle costs reflect the addition of the operations, maintenance, and D\&D costs to the capital costs. The operation and maintenance costs include the specific work crews based on 24-7 operation over a five year period. The major chemical consumables and utility costs are based on the quantities developed from the process layouts and equipment list. The cost profiles were input using estimator experience and judgment. There was no specific attempt to optimize the cost profiles in the six estimates. The project schedule was used for escalating costs.

The estimates represent a Class 4 level of project definition as defined by the Association for the Advancement of Cost Engineering (AACE) International. A Class 4 estimate has an expected accuracy range from a minus $30 \%$ to a plus $50 \%$.

Cross flow filtration requires the same throughput capacity when paired with IX-sRF and CSSX. However, when paired with FC, CFF requires a $450 \%$ increase in the number of filtration tubes. Rotary micro-filtration requires the same throughput capacity when paired with IX-sRF and CSSX, but requires a $300 \%$ increase in the number of filtration modules when paired with FC. In addition, the number of RMF modules required when paired with FC precludes installation of the modules in a DST, requiring the construction of an additional below grade vault in lieu of installation of the above grade pit in the other two pairings.

It is noted in RPP-RPT-37751 that the assumed design life of the rotary micro-filters is three years. An out-year operating cost for replacement of the RMF modules has been included in the fourth operating year. Consequently, within the same cesium separation technology pairing, the capital cost of rotary micro-filtration exceeded the capital cost of cross-flow filtration.

Of the two solids filtration technologies, the lowest project capital cost and lowest life-cycle cost result from pairing CFF with IX-sRF. Both the highest capital cost and highest life cycle cost result from pairing $\mathrm{RMF}$ with $\mathrm{FC}$.

The individual evaluations of Cost Impacts for each of the entrained solids filtration technologies can be found in CFF-5.1.1 through CFF-5.1.3 and RMF-5.1.1 through RMF-5.1.3.

\subsubsection{Schedule Impacts}

In addition to cost, it is very important to understand what, if any, impact the candidate IPS technologies will have on the IPS implementation schedule. A major question that needs to be answered is whether the IPS project can be completed in a timely manner so as to allow WTP's LAW Vitrification facility to operate for a significant period of time prior to startup of WTP's Pretreatment facility. Understanding how the candidate technologies impact that issue will be key to deciding whether it is worthwhile to go forward with this project. 
The IPS is a major acquisition whose processes must be developed and demonstrated and whose facility must be designed, constructed, permitted, tested and operated in a manner that meets DOE, Federal, State and local requirements and regulations. The major programmatic activities that must be conducted for the IPS project are: engineering and design, regulatory and permitting activities, technology maturity achievement, facility construction, equipment procurement and installation, startup/readiness, and cold/ hot operation. Some of these activities are interrelated and have significant impact on the overall IPS schedule.

In order to better assess the schedule impacts of the candidate technologies, three specific areas of concern were identified, as well as evaluating the overall schedule confidence. The three selected areas were nuclear safety and licensing, permitting, and the D\&D activity. With respect to the solids removal technologies, their respective impacts to the IPS implementation schedule are discussed in the sections below.

\subsubsection{Overall Schedule Confidence}

An implementation schedule for IPS has been developed using estimated durations and logic ties for six major activities - design, testing, permitting, safety and licensing, construction, and startup. This schedule also included the four Critical Decision (CD) milestones (CD-1, CD-2, CD-3, and CD-4) as well as the milestone for the expected issuance of the TC\&WM EIS ROD (expected January 2010).

Based on the technology-specific duration estimates for each of the major activities, it was apparent that differences in durations for the two filtration technologies (CFF and RMF) were rather minor when compared with those for the cesium separations technologies. As a result three scheduling cases have been developed, one for each of the cesium separations technologies, all five of which include "generic" filtration. Therefore, overall schedule confidence is discussed in Section 4.5.2.1.

The individual evaluations of Overall Schedule Confidence for each of the entrained solids filtration technologies can be found in CFF-5.2.1 and RMF-5.2.1.

\subsubsection{Licensing}

The primary area of concern for the nuclear safety analysis will be the high operating speed of the RMF unit and the installation of these units into a 42" DST riser in the feed tank (AP-104). Savannah River has performed extensive tests on the RMFs up to 48 " in diameter, but has not fully deployed an operational RMF unit. Because there is less Hanford-specific knowledge and experience with the RMF process and the equipment has not been fully deployed here or elsewhere, there is higher probability that the IPS safety analysis will impact on-time completion of the IPS design.

The primary area of concern for nuclear safety analysis process will be the high axial flow rates of liquid waste through the CFF filter housing and the operating pressures across the CFF filter media. However, there is considerable experience with this filtration process at several other DOE sites, including West Valley, Savannah River, and Oak Ridge, and it has been extensively incorporated into the WTP design. Therefore, the IPS schedule for completing its safety analysis should not be impacted by the CFF process.

The individual evaluations of Licensing for each of the entrained solids filtration technologies can be found in CFF-5.2.2 and RMF-5.2.2.

\subsubsection{Permitting}

The key events in assessing whether there will be any schedule impact from either the CFF or the RMF processes is completion of the TC\&WM EIS (expected December 2009) and issuance of its Record of 
Decision (expected January 2010). Based on the ROD, an evaluation of the CFF and RMF technologies will need to be performed to determine whether they are adequately covered or additional NEPA coverage is required (e.g., EA). Once the NEPA process is completed, the draft RCRA permit can be issued for public review and comment by the regulatory agency. The RCRA Part B permit must be approved before IPS construction can start. In accordance with the Tri-Party Agreement, the overall schedule for the permitting process is $28-33$ months. Because solids filtration is a preliminary process step that does not generate waste streams requiring disposal, it is unlikely that either of these technologies will cause significant delays in the RCRA Part B permitting process.

Assuming that the TC\&WM EIS is issued in December 2009 as expected, permitting of the solids filtration process does not appear to impact IPS start of construction. However, in the event that completion of the TC\&WM EIS is significantly delayed beyond its expected completion date of December 2009, an alternate NEPA option may be required (e.g., an Interim Action EA).

The individual evaluations of Permitting for each of the entrained solids filtration technologies can be found in CFF-5.2.3 and RMF-5.2.3.

\subsubsection{Decontamination and Decommissioning}

Because the CFF equipment will be installed in a newly constructed facility, appropriate considerations for accommodating its eventual D\&D will be included during the design phase of IPS. The D\&D of this equipment will most likely occur as part of the IPS D\&D activity following completion of the IPS mission. It is anticipated that any design activity in support of eventual D\&D of the CFF equipment will have negligible impact on the IPS implementation schedule.

Although the RMF equipment will be installed in a 42" D riser in AP-104, rather than in a newly constructed facility, its eventual D\&D can also be appropriately accommodated during the design phase of IPS. D\&D of this equipment will occur, either when new retrieval equipment needs the 42 " riser, or at the end of the TWRS mission as part of the AP-104 tank closure. It is anticipated that any design activity in support of eventual D\&D of the RMF equipment will have negligible impact on the IPS implementation schedule.

The individual evaluations of Decontamination and Decommissioning for each of the entrained solids filtration technologies can be found in CFF-5.2.4 and RMF-5.2.4.

\subsubsection{Double-shell Tank Space}

DST volume is a critical resource in the management of Hanford tank waste. Such space is limited and must be managed with care. All liquid waste pretreatment activities must be carefully evaluated for their potential impact on DST space. From the DST space management point of view, the total net volume of DST space needed or freed up and the annual rate at which DST space is consumed or made available are important metrics. Impacts of entrained solids filtration technologies on the DST space, while considered to be relatively minor, are discussed below.

Removal of entrained solids is a first step in the IPS process for the preparation of LAW feed to the WTP. This step only becomes necessary if one of the cesium separation processes is implemented. Entrained solids removal or filtration processes are essentially passive processes in that neither significantly reduces the radioactivity of the liquid waste nor adds any significant amount of external resources (chemicals, water, etc.) to achieve solids separation.

Two filtration process activities impact DST space. The first is the volume of DST space required to accommodate the concentrated solution of filtered solids. The second is the volume of DST space 
required for the filter cleaning solution that is sent back to the DST. However, the total impact of these two filtration processing steps is relatively minor and should have no significant impact on the net rate at which DST space will become available. The rate change of the DST volume is only realized when the filtrate is removed and processed through a cesium removal process. The rate at which DST space is freed up and the total volume of DST space that will become available as a result of the IPS processing will be discussed in Section 4.

While the actual concentration of suspended solids in the candidate tanks is thought to be much less, for this study it has been assumed that these wastes contain 0.5 weight percent solids on average. It is further assumed that both filtration processes (cross-flow filtration and rotary micro-filtration) remove essentially all of the suspended solids, and for purposes of evaluating the impact on DST space that these solids streams would be concentrated to the waste transfer limit of 20 weight percent solids. Assuming that the density of the suspended solids is $1.4 \mathrm{~g} / \mathrm{cc}$ and given that the total volume of waste in the five candidate tanks for IX-sRF and CSSX is slightly more than 5 million gallons, this will result in a "worst case" estimate of $\sim 100,000$ gallons of suspended solids at $20 \mathrm{wt} \%$ being stored in the AP-104 feed tank after the waste from all five tanks has been processed in IPS. Because the FC option requires 16 batches, or approximately $3 \mathrm{X}$ the volume of waste feed, the estimated volume of stored solids for this option is $\sim 300,000$ gallons.

Both the cross-flow filters and the rotary micro-filters will require periodic cleaning and backwashing to maintain their effectiveness. The recommended cleaning regimen for the CFF and RMF is monthly cleaning and backwashing with $0.1 \underline{\mathrm{M}} \mathrm{NaOH}$ and a semiannual cleaning with $2 \underline{\mathrm{M}}$ nitric acid. During a nominal five-year operation of IPS, it would require $\sim 15 \mathrm{~K}$ gallons of $\mathrm{NaOH}$ and $\sim 2.5 \mathrm{~K}$ gallons of nitric acid, if either the IX-sRF or CSSX technologies were selected, and $\sim 45 \mathrm{~K}$ gallons of $\mathrm{NaOH}$ and $\sim 7.5 \mathrm{~K}$ gallons of nitric acid for the FC technology. Worst case, this would result in a total of $\sim 54 \mathrm{~K}$ gallons of neutralized cleaning liquids being sent to the DST system, or $\sim 11 \mathrm{~K}$ gallons per year for cleaning and back flushing the cross-flow filters used to support the Fractional Crystallization process.

The individual evaluations of Double-shell Tank Space for each of the entrained solids filtration technologies can be found in CFF-5.3.1, CFF-5.3.2, RMF-5.3.1, and RMF-5.3.2.

\subsubsection{Impacts to WTP and Supplemental Treatment Plants}

Because the intent of the IPS facility is to provide pretreated wastes to WTP's LAW facility and to Supplemental Treatment for immobilization, it is important to understand the impacts, positive or negative, of the IPS operation on these facilities. These impacts are driven by the quantity and characteristics (chemical conditions, radionuclide inventory, chemical components, etc.) of products delivered by the IPS to these facilities. These impacts are qualitatively measured in several ways, such as production rates, WTP LAW mission duration, number of high- and low-level packages, lessons learned, technology transfer, ALARA, diversity of technology and positive programmatic impacts and opportunities. These impacts are qualitatively assessed below for the two solids filtration technologies, namely $\mathrm{CFF}$ and $\mathrm{RMF}$.

\subsubsection{Production Rate Impact}

Glass production rate is dependent on the feed composition, as well as some specific chemical constituents which may influence the glass quality and/or the melting rate of the glass. Since solids filtration is a precursor to the cesium separation process, it does not establish the rate at which feed is delivered, so the two candidate filtration technologies have essentially no impact on the production rate for WTP LAW and Supplemental Treatment facilities. Removed entrained solids will be returned to the DST system for subsequent processing with WTP high-level waste (HLW) feed. Because these solids 
would otherwise have been removed by WTP's pretreatment facility, they have no adverse impact on the WTP HLW facility production rate.

The individual evaluations of Production Rate Impact for each of the entrained solids filtration technologies can be found in CFF-5.4.1 and RMF-5.4.1.

\subsubsection{Mission Duration}

The filtration process is a preliminary step to pretreatment that removes entrained solids from the liquid waste. Filtration is required to support the subsequent cesium separation process, not LAW treatment. Therefore, the filtration process has no impact on the duration of the LAW treatment mission. The filtered solids will be returned to the DST system and stored until the WTP Pretreatment and HLW facilities become operational. These filtered solids will then be transferred to WTP, along with other tank wastes for pretreatment and HLW immobilization. "Worst case" estimates for the volume of filtered solids removed from the 5 candidate feed tanks is $\sim 100 \mathrm{~K}$ gallons (at the waste transfer limit of $20 \mathrm{wt} \%$ ) for the IX-sRF and CSSX technologies and $\sim 300 \mathrm{~K}$ gallons from the 16 feed batches required for FC. Since vitrification of these filtered solids is a part of the current WTP mission, neither one of the filtration technologies impact the overall LAW mission duration of the WTP.

The individual evaluations of Mission Duration for each of the entrained solids filtration technologies can be found in CFF-5.4.2 and RMF-5.4.2.

\subsubsection{Number of High and Low-Level Packages}

Entrained solids filtration is the first of a two processes implemented for pretreatment in the IPS. The entrained solids product stream that is created by filtration will be stored in the feed tank (AP-104) until it is eventually transferred to WTP for pretreatment and vitrification. Once these filtered solids are transferred to WTP, they will be pretreated and immobilized as HLW, along with other tank wastes. "Worst case" estimates for the volume of filtered solids removed from the 5 candidate feed tanks is $\sim 100 \mathrm{~K}$ gallons (at the waste transfer limit of $20 \mathrm{w} / \mathrm{o}$ ) for IX-sRF and CSSX and $\sim 300 \mathrm{~K}$ gallons from the 16 batches required for FC. Therefore, the filtered solids resulting from IPS processing will produce some small number of IHLW packages at WTP. However, because these entrained solids would have otherwise been filtered by WTP Pretreatment and subsequently vitrified at WTP HLW, there is no net impact on the HLW packages produced by the WTP. Also because both filtration technologies assume $99.99 \%$ solids removal efficiency, there is no difference between the two candidate technologies.

The individual evaluations of Number of High and Low-Level Packages for each of the entrained solids filtration technologies can be found in CFF-5.4.3 and RMF-5.4.3.

\subsubsection{Lessons Learned Benefits for WTP Pretreatment}

CFF has been selected as the baseline filtration technology for WTP Pretreatment. The operation and maintenance of the CFF equipment in IPS will provide valuable data and experience for the startup and operation/maintenance of similar equipment in WTP's Pretreatment facility. The WTP pretreatment facility has established a technology verification program for the CFF deployment. Early deployment of $\mathrm{CFF}$ in IPS can become an integral part of this verification program. With IPS coming on line several years earlier than the WTP Pretreatment facility, there will be opportunities to make programmatic adjustments to the CFF deployment program, if necessary. This synergetic process may provide some cost benefits to the overall Hanford tank waste program. Conversely because RMF is not being used in WTP, its deployment, operation and maintenance in IPS would not provide any lessons learned benefits to the WTP. 
The individual evaluations of Lessons Learned Benefits for WTP Pretreatment for each of the entrained solids filtration technologies can be found in CFF-5.4.4 and RMF-5.4.4.

\subsubsection{Technology Transfer to WTP}

Because CFF has already been selected as the baseline filtration technology for WTP Pretreatment, no technology transfer to WTP will occur from this technology. However, the technology deployment and operation and maintenance of the RMF equipment in IPS will provide valuable data and experience for potentially transferring this technology to WTP in the event that the CFF baseline technology does not meet WTP specifications for solids removal.

The individual evaluations of Technology Transfer to WTP for each of the entrained solids filtration technologies can be found in CFF-5.4.5 and RMF-5.4.5.

\subsubsection{ALARA}

The primary source of radioactivity in the supernatant liquids is ${ }^{137} \mathrm{Cs}$. Transuranics and ${ }^{90} \mathrm{Sr}$ play a very limited role in the ALARA program for the WTP facility. The filtered solids will be returned to the WTP for further treatment and vitrification in its $\mathrm{HLW}$ facility. The small amount of radioactivity that is present in the returned entrained solids has not been estimated in the mass balance spreadsheets, so its eventual impact to WTP cannot be quantified at this time. However, WTP HLW facility is designed to accept the solids and sludge from the tank waste, the entrained solids removed by either of the candidate filtration technologies will likely not change the ALARA design basis of the WTP or Supplemental Treatment Plant.

The individual evaluations of ALARA for each of the entrained solids filtration technologies can be found in CFF-5.4.6 and RMF-5.4.6.

\subsubsection{Diversity of Technology}

CFF has been selected as the baseline filtration technology for WTP Pretreatment. Therefore, the use of CFF in IPS does not initially provide an opportunity for diversifying WTP's technology portfolio. Conversely RMF provides a diversity of technologies at Hanford for tank waste treatment. In the event that significant problems are encountered with CFF during early WTP pretreatment facility, there would be a possibility to use the RMF system installed for entrained solids removal either in the AP-104 feed tank or in the IPS facility, prior to transferring the waste to the WTF pretreatment facility. Therefore, the use of RMF in IPS does provide diversity of technology for Hanford Tank waste programs and to WTP pretreatment activities.

The individual evaluations of Diversity of Technology for each of the entrained solids filtration technologies can be found in CFF-5.4.7 and RMF-5.4.7.

\subsubsection{Positive Programmatic Impacts and Opportunities}

Deployment of CFF technology in IPS will provide opportunities to reduce some of the costs that the WTP Program would otherwise have to spend on CFF development and demonstration. For example, full-scale use of CFF in IPS will provide performance data on this technology that WTP would otherwise obtain through its pilot-scale testing program. Furthermore, the IPS experience will be with radioactive wastes, rather than cold simulants, so that technical uncertainties about CFF performance are significantly reduced. The experience gained from the use of CFF in IPS will also reduce the uncertainty in WTP's cost estimates for operation and maintenance of this system, and may even allow some acceleration in WTP's startup schedule for its Pretreatment facility. 
Because RMF is not the baseline technology for solids filtration in WTP or Supplemental Treatment, there are no programmatic impacts or opportunities envisioned with this technology. However, in the event that performance of the baseline solids filtration technology proved to be unsatisfactory, the knowledge gained from RMF's deployment in IPS would provide valuable input to a recovery plan for WTP's solids filtration process.

The individual evaluations of Positive Programmatic Impacts and Opportunities for each of the entrained solids filtration technologies can be found in CFF-5.4.8 and RMF-5.4.8.

\subsubsection{Impacts to other facilities (ETF, laboratory)}

The selected filtration process may potentially impact other Hanford Site support facilities. In this assessment, impacts to facilities such as the analytical laboratories (222-S Analytical Laboratory and WTP's Analytical Laboratory), the Effluent Treatment Facility (ETF), and the 242-A Evaporator operations are evaluated and summarized below.

\subsubsection{Analytical Equipment, Methods and Capacity}

There is currently no requirement to sample and analyze the filtered liquid (filtrate) prior to its transfer to the cesium removal process for either CFF or RMF. To ensure that the concentration of the solids in the waste stream being returned to AP-104 from the CFF process does not exceed the DST waste transfer limits, it will be measured in-line. This type of measurement does not require any new analytical method. Since the RMF process is performed at-tank, the wt \% solids in the waste stream being returned to AP104 will not be measured. Therefore, there is no anticipated impact on the analytical services at either WTP's Analytical Laboratory or the 222-S Analytical Laboratory.

The individual evaluations of Analytical Equipment, Methods and Capacity for each of the entrained solids filtration technologies can be found in CFF-5.5.1 and RMF-5.5.1.

\subsubsection{Compliance to ETF WAC}

Both the CFF and RMF are closely coupled with the feed tank (AP-104). In both systems, a continuous recirculation system is used to bleed the feed to the filtration unit. Any solution generated due to filter washing, back flushing, or cleaning is routed directly back into that tank. Eventually those small volumes will be passed through as part of the clarified feed to the cesium separation process and will be included in whatever fraction of waste the cesium separation process sends back to the DSTs as a cesium-loaded stream. Therefore, both filtration systems do not generate any effluent stream requiring ETF services and thus have no impact on the ETF operation.

The individual evaluations of Compliance to ETF WAC for each of the entrained solids filtration technologies can be found in CFF-5.5.2 and RMF-5.5.2.

\subsubsection{ALARA}

Because CFF is being incorporated into a new facility for IPS, the ALARA principle will be proactively addressed during the facility and process design to ensure that Waste Acceptance Criteria for supporting facilities are met, less hazardous/less toxic materials that would potentially impact supporting facilities are used wherever possible, and sampling/analysis requirements at the analytical laboratories are minimized. Alternatively because RMF is being incorporated into an existing Double-Shell Tank (AP104 ), it may be more challenging to incorporate the ALARA principle with respect to these same support facilities. 
The individual evaluations of ALARA for each of the entrained solids filtration technologies can be found in CFF-5.5.3 and RMF-5.5.3.

\subsubsection{Evaporator}

Neither the CFF nor the RMF processes send any waste stream directly to 242-A Evaporator. Any incidental process waste created by either of these processes will be collected and sent to 242-A Evaporator as a combined facility waste stream. Contributions from the CFF and RMF processes are anticipated to be minimal. Based on this, neither of the entrained solid separation technologies have any measurable impact on the 242-A Evaporator operation.

The individual evaluations of Evaporator for each of the entrained solids filtration technologies can be found in CFF-5.5.4 and RMF-5.5.4.

\subsubsection{Resources and Materials}

An assessment of processes was made to look at resource and material specialty required by each filtration processes for an overall implementation. Engineering resources are assumed to be at a premium for implementation of such a project such as the IPS at the Hanford Site when other large projects are also being implemented. But such resource competition will exist regardless of the technology selected.

Rotary micro-filtration technology will require some additional technology development personnel resources. This is due to re-engineering that must be performed to the design developed by Savannah River Site. Hanford tanks have a 42 " riser where as SR tanks have 48 " risers. In addition, only a single vendor exists who has had experience in building such a filtration unit to meet DOE requirements and specifications. Such engineering conditions require proper planning and approach for a minimized risk implementation.

Conversely, the CFF unit can be assembled by multiple experienced vendors requiring limited technological oversights. Such units have been produced in the past and are being developed for the WTP project. Cross-flow filtration system requires a very high capacity recirculation pump $(1,000-3,000$ $\mathrm{gpm}$ ). Such large pumps have been developed and can be implemented with proper engineering design and fabrication oversight.

Both filtration technologies do not require any special or unusual material of construction requiring special precautions or procurement conditions. But vendors qualified to fabricate nuclear grade equipment may be difficult to find. Such problem becomes a larger issue when only a single source is available for fabricating any equipment. Due to a single vendor source for the rotary micro-filtration, some pricing risk does exist for the future.

The individual evaluations of Resources and Materials for each of the entrained solids filtration technologies can be found in CFF-5.6.1, CFF-5.6.2, RMF-5.6.1 and RMF-5.6.2. 


\subsection{CESIUM SEPARATION ASSESSMENTS}

Three technologies (fractional crystallization, caustic-side solvent extraction, and ion exchange) have been identified for use within the IPS to reduce cesium concentrations. This section summarizes the Assessment Form information details for each decision line, compiled in RPP-RPT-37741 (CH2M HILL 2008d). Individual Assessment Form numbers are noted below with the Assessment form designator, e.g., FC-1.1.1; no specific reference back to RPP-RPT-37741 (CH2M HILL 2008d) is included with this designation, but is implied.

\subsection{SAFETY}

Three measures, Process Safety, Criticality Safety, and Industrial Safety and Hygiene have been identified for this Criterion. The safety assessment discussion is provided below.

\subsubsection{Process Safety}

Eight different process safety definitions (Attachment A) have been defined to assess overall impact of the three cesium separation technologies on various process safety aspects, including nuclear safety, chemical safety, fire safety, operational safety. These safety issues are covered by looking at various aspects of Materials at Risk (MAR), process stability, chemical reactivity, fire and operational hazards.

\subsubsection{Quantity of Material at Risk}

This process safety definition evaluate whether there is difference in the quantity of MAR among the three options. Less material at risk would be better for this definition. The quantities of radiological and chemical material (in curies for radionuclides and in grams for chemicals) available to be acted upon by a given physical stress should be evaluated.

Using radiological data and process data from mass balance calculations, the radiological MAR was calculated for the Feed Receipt tank, Cesium Product tank, and LAW Product tank for each technology. The intent of this assessment was to gain knowledge about the MAR at key component locations. Activities at these three locations were calculated and are provided with each individual assessment for each respective technology.

The quantity of MAR based on IPS inventory is related to the concentrations and volumes of the primary lag storage vessels. Fractional Crystallization has the largest Feed Receipt tank; however, it is has the smallest LAW Product tank and least radioactivity in the LAW product stream. The CSSX and IX-sRF processes have similar sized Cesium Product tanks.

The FC process does not use any chemicals and therefore it has no additional chemical MAR. Toxicological doses are driven by the chemicals in the DST wastes and not process chemicals. The CSSX and IX-sRF processes use various chemicals and MAR based on chemical storage is listed in Table 4-1.

The individual evaluations of Quantity of Material at Risk for each of the Cs separation technologies can be found in CSSX-1.1.1, FC-1.1.1, and SRF-1.1.1.

Table 4-1 - Chemical MAR based on Chemical Storage 


\begin{tabular}{|c|c|c|}
\hline \multirow{2}{*}{ Chemical } & \multicolumn{2}{|c|}{ Storage volume, gallons } \\
\hline & CSSX Process & sRF Ion Exchange \\
\hline Bulk NaOH, $50 \mathrm{wt} \%$ & 12,500 & 12,500 \\
\hline $0.01 \underline{\mathrm{M} N a O H}$ & 1,000 & 2,000 \\
\hline $0.5 \underline{\mathrm{M} \mathrm{NaOH}}$ & ----- & 2,700 \\
\hline Bulk $\mathrm{HNO}_{3}$ & 55 gal drum & 6,500 \\
\hline $0.05 \mathrm{MHNO}_{3}$ & 1,000 & ----- \\
\hline $0.001 \mathrm{MHNO}_{3}$ & 1400 & $\begin{array}{ll}---- \\
\end{array}$ \\
\hline $0.45 \mathrm{M} \mathrm{HNO}_{3}$ & ------ & 12,000 \\
\hline Bulk $\mathrm{NaNO}_{2}, 20 \mathrm{wt} \%$ & $2-55$ gal drums & 55 \\
\hline Organic Solvent & 550 & ----- \\
\hline sRF resin & $\begin{array}{ll}---- \\
\end{array}$ & $\sim 600$ \\
\hline
\end{tabular}

\subsubsection{Concentrations of MAR}

Evaluation of the concentration of radiological (unit liter dose) and chemical (unit sum of fractions) MAR is important because it is the amount of material available to be acted on by a given physical stress.

Lower concentrations of MAR would be better for this definition.

For radioactive MAR unit liter dose (ULD) values provide comparisons of concentrations of MAR. These concentrations of MAR were computed at isolated steps in each process. Comparisons to ULDs of other processes at similar steps provide a comparative assessment of the technologies. The ULDs of the Cs-depleted waste stream being sent to WTP from all three cesium separation technologies are very low and in line with each other. Detailed tabular list ULDs of various streams for each technology is provided in CSSX-1.1.2, FC-1.1.2, and SRF-1.1.2.

ULDs for cesium-loaded streams being returned to the DST system are similar for CSSX (66 to 465) and FC (99 to 516) and IX-sRF (155 to 342$)$ technologies.

Chemically for the CSSX process, the highest concentration/TEEL ratio $\left(1.53 \times 10^{7}\right)$ was calculated for 50 $\mathrm{wt} \% \mathrm{NaOH}$. The ratios and sum of fractions for the IPS streams are within the bounds of similar chemical analyses performed for tanks in the tank farms with respect to evaluation of toxicity.

Relatively speaking, there are insignificant differences among the three cesium separation processes with respect to ULD values for the LAW to WTP product stream. The individual evaluations of Concentrations of MAR for each of the Cs separation technologies can be found in CSSX-1.1.2, FC-1.1.2, and SRF-1.1.2.

\subsubsection{Dispersability of MAR}

Dispersability is the lowest with solids and increases from liquids to powders to gases. Less dispersible forms would be better for this definition. MAR remains in a liquid phase for all three cesium separation technologies. This is a favorable state when considering the form of the MAR as related to dispersability.

In the IX-sRF process, during certain steps a major MAR constituent, radioactive cesium is ionically bound to the sRF resin. After that ${ }^{137} \mathrm{Cs}$ is eluted and sent to the DST system in a liquid form. Throughout the CSSX process, the radiological MAR remains in a liquid phase, either as an aqueous or organic stream. The MAR is returned to the DST as an aqueous stream. The radiological MAR for the 
FC process remains entrained in liquid; however some of the MAR can become entrained in the vapor phase.

The individual evaluations of Dispersability of MAR for each of the Cs separation technologies can be found in CSSX-1.1.3, FC-1.1.3, and SRF-1.1.3.

\subsubsection{Dispersive Energy}

For dispersive energy inherent in process parameters (e.g., heat, off-gas, pressure), internally process initiated events were looked at for these three cesium separation technologies. Focused was placed on internally initiated events and process-initiated events only. Consideration can be given to parametric factors such as temperature, flow rate, pressure, kinetic energy, etc. Various types of dispersive energies were reviewed. They were: kinetic energy, reactive chemical energy, thermal potential energy, thermal energy and potential energy-gravitational and organic solvent.

A detailed assessment for the CSSX process is provided in the assessment summary form CSSX-1.1.4. Most forms of energy generated in the CSSX are with predictable contributions to dispersive energy (e.g., centrifugal contactors). Reactive chemical energy comes from chemicals present in the CSSX process. Reactivity of chemicals ranges from moderate to extreme for these chemicals when they come in contact with other materials or chemicals. The CSSX solvent is made of four different components, as described in Section 2.2.2.2. One of these four components is Isopar ${ }^{\circledR} \mathrm{L}$. This is a semi-volatile component and combustible with a flash point of $144{ }^{0} \mathrm{~F}$. This chemical presents the potential for thermal transients.

A detailed assessment for the IX-sRF process is provided in the assessment summary form SRF-1.1.4. Four specific types of dispersive energies were identified - kinetic, reactive chemical, potential thermal, and potential gravitational. Rotational energy generated by pumps (5-100 gpm capacity) is standard and does not create any unusual condition. The chemicals used are $\mathrm{NaOH}, \mathrm{HNO}_{3}$ and $\mathrm{NaNO}_{2}$. These chemicals are mainly used in the rinse, elution and regeneration activities. Reactivity can range from moderate to extreme for these chemicals when they come in contact with other materials and chemicals. Hydrogen gas can be generated due to radiolysis that occurs in the ion exchange column. While hydrogen and oxygen are the primary gaseous products of radiolysis, small amounts of $\mathrm{CO}_{2}, \mathrm{CO}, \mathrm{CH}_{4}$, and $\mathrm{N}_{2} \mathrm{O}$ are also produced. Off-gassing may also occur from inadvertent resin exposure to high acid concentrations, but is unlikely for the IX-sRF process. Potential gravitational energy from tanks and vessels can be converted to dispersive energy if a leak or rupture should occur.

Fractional Crystallization is a stage-wise separation technique that relies upon liquid-solid phase transition and enables multi-component mixtures to be split into narrow fractions, ultimately leading to concentration of selected components, through the virtue of selectivity found in solid-liquid equilibrium. Because this process is based on selective evaporation and cooling, dispersion energy is minimal. Process steam provides the majority of dispersive energy input to the process. Transfer pumps (the FC recirculation pumps are rated for over $5000 \mathrm{gpm}$ ), tanks, and vessels also provide potential energy that can be converted to dispersion energy in the form of free-fall spills, sprays, etc.

The FC process does not create such hazard as nitric acid is not used within the process in the facility. The individual evaluations of Dispersive Energy for each of the Cs separation technologies can be found in CSSX-1.1.4, FC-1.1.4, and SRF-1.1.4.

\subsubsection{Process Stability}

The assessment scope for this definition includes qualitative evaluation of the inherent process stability, in particular the proposed control system, method of shutdown, and ease of shut down. Easier and faster shut down is better as it improves quickness of response in an emergency condition. 
Process stability is measured by ensuring that safe shutdown can be achieved in a quick and safe manner. The $\mathrm{FC}$ process operates under vacuum conditions and at modest temperature of approximately $60^{\circ} \mathrm{C}$, thereby allowing rapid short-term shutdown, if necessary. Thermal mass of the FC unit is large at a slightly elevated temperature. Even after the stopping of boiling (which can be achieved easily by shutting off the reboiler steam or decreasing the vacuum), solids formation continues when the solution starts to cool. Such conditions may create a difficulty to restart the process if not properly controlled by dumping or flushing the system. Longer-term shutdowns will require flushing to prevent significant build up in the FC units.

The IX-sRF process operates under a slight pressure and at $25^{\circ} \mathrm{C}$. The flow to the columns shuts down simply by turning off the pump that feeds the ion exchange columns; therefore, the process can quickly be shutdown. A potential concern is the heating of the column caused by the ${ }^{137} \mathrm{Cs}$ decay heat, if it remains shut down for an extended period of time. Proper design of tank sizing with emergency rinse and elution cycle should be included to allow a safe shutdown condition. Once the cesium is removed the columns will remain in a safe condition.

The CSSX process also operates under a slight pressure and at $25^{\circ} \mathrm{C}$. This is continuous process and therefore, shutdown and start-up are more complicated. Controlled shutdowns that include removal of most of the ${ }^{137} \mathrm{Cs}$ from the organic prior to draining it to the organic feed tank can be accomplished in a few hours. In an emergency, immediate shutdown can be achieved with ${ }^{137} \mathrm{Cs}$ laden organic in the organic storage tank.

The FC process does not create such hazard as nitric acid is not used within the process in the facility. The individual evaluations of Process Stability for each of the Cs separation technologies can be found in CSSX-1.1.5, FC-1.1.5, and SRF-1.1.5.

\subsubsection{Tank Farm Hazard}

Selected process must not create any new (or minimal) TF operation hazard or exacerbate an existing TF Hazard. Based on the TF DSA defined accident scenarios for hazard evaluation, potential TF hazardous conditions were collected into candidate accident groups sharing similar accident phenomenology for this assessment.

Based on these defined scenarios, three cesium separation technologies were evaluated in a qualitative manner for their potential in creating hazards specifically to the TF operation only. Their potentials TF impacts are shown below in Table 4.2 . 
Table 4-2 - Potential Tank Farm Hazards from Cesium Separation Technologies

\begin{tabular}{|l|c|c|c|}
\hline \multirow{2}{*}{$\begin{array}{c}\text { Tank Farms Representative Accident } \\
\text { Scenarios }\end{array}$} & \multicolumn{3}{|c|}{ Potential to Impact on Accident/ Hazard } \\
\cline { 2 - 4 } & FC & CSSX & IX-sRF \\
\hline Flammable Gas Accidents & No & No & No \\
\hline Nuclear Criticality & No & No & No \\
\hline Vacuum Exhaust Line Rupture & No & No & No \\
\hline Release from Contaminated Facility & No & No & No \\
\hline Tank Failure Due to Excessive Loads & No & No & No \\
\hline Above Ground Structure Failure & No & No & No \\
\hline Mixing of Incompatible Materials & No & Yes & Yes \\
\hline Waste Transfer Leak & No & No & No \\
\hline Unplanned Excavation / Drilling & No & No & No \\
\hline
\end{tabular}

All three cesium separation technologies do not create any TF hazards for flammable gas accidents, nuclear criticality, vacuum exhaust line rupture, release from contaminated facility, tank failure due to excessive loads, above ground structure failure, waste transfer leak, and unplanned excavation /drilling.

Mixing of incompatible materials The CSSX process uses nitric acid and sodium hydroxide.

Additionally, CSSX uses a four-component organic mixture from which trace amounts will be lost in the product and waste streams. Controls to prevent introduction of these chemicals / compounds into the waste tanks needs to be further evaluated. Introduction of such incompatible materials with tank waste could exacerbate the existing TF hazards.

The IX-sRF process uses nitric acid and sodium hydroxide during the rinse, elution, and regeneration phases of the operating cycle. Degradation of the SRF resin, which has the potential for introduction of the resin into the product and waste streams, is also anticipated. Introduction of such incompatible materials with tank waste could exacerbate the existing Tank Farms hazards. In both these processes, proper safeguards must be installed to ensure full compliance with TF DSA. This may include, but not limited to, $\mathrm{pH}$ adjustment, corrosion inhibitor adjustment, sampling and verification, etc. prior to transfer to DST.

The FC process does not create such hazard as nitric acid is not used within the process in the facility. The individual evaluations of Tank Farm Hazard for each of the Cs separation technologies can be found in CSSX-1.1.6, FC-1.1.6, and SRF-1.1.6.

\subsubsection{Fire Hazard}

A system and/or facility requiring little or no flammable material or condition is more suitable from the fire safety point of view. Many things may lead to fire concerns. Some of those are combustible or explosive material, production of flammable gas or by products, high heat system, etc. Combustible loading inherent to each cesium separation technology was qualitatively assessed in this definition.

Normal operation of CSSX uses a four-component organic solvent. The solvent consists of an extractant dissolved in an inert hydrocarbon matrix (Isopar L). Isopar L has a low flashpoint of $62^{\circ} \mathrm{C}$, which gives it an NFPA flammability hazard of 2 . The organic solvent is stored in a 550-gallon Solvent Feed Tank. The organic solvents are also susceptible to forming hydrogen gas due to radiolysis. Due to flammability of this organic solvent, fire safety considerations will be required during the design and operation of the facility. 
Fractional crystallization process does not use any organic solvent or combustible material. Elevated temperatures can increase flammable gas due to radiolysis and chemical reactions.

In the IX-sRF process, the ion-exchange columns used in the process may generate hydrogen gas as a result of radiolysis. Additionally, the process uses chemicals that must be managed to prevent mixing, which could result in heat generation.

The individual evaluations of Fire Hazard for each of the Cs separation technologies can be found in CSSX-1.7, FC-1.1.7, and SRF-1.1.7.

\subsubsection{Reactive Chemicals}

Each process was evaluated for its use of reactive chemicals, both organic and inorganic. A process with fewer reactive chemicals is better from a safety perspective. Review of process flow sheets for the three cesium separation technologies identified the following chemicals of reactivity concern:

- FC process: No active chemicals for processing, may be nitric acid for cleaning

- CSSX process: Nitric acid, sodium hydroxide, Isopar ${ }^{\circledR}$ L, BOBCalixC6, TOA

- IX-sRF process: Nitric acid, spherical Resorcinol-Formaldehyde (sRF) resin

For all three processes use of nitric acid has a potential for chemical reactivity. Its use must be carefully monitored and evaluated, as some of that nitric acid containing solution may be returned to the TF.

In the IX-sRF process, the spherical Resorcinol-Formaldehyde resin will degrade and some of that resin may end up in product and waste streams. However, resin behavior has been evaluated by SRNL for the WTP Project and resin degradation is not anticipated to create a safety issue. Reactions between nitric acid and caustic streams must be carefully monitored.

Similar caustic and nitric acid reactions need to be carefully monitored in the CSSX process. In addition to those interactions, organics used in this process must be assessed. ISOPAR L is the main organic used in the process by weight and volume. It has a flash point of $62{ }^{\circ} \mathrm{C}$, NFPA flammability hazard or 2 , but has a 0 reactivity hazard. The Calix 4 has a reactivity of 1 while the TOA has an instability rating of 0 . Even though TOA by itself is incompatible with strong acid/oxidizer, both of these chemicals are used in the process at low concentrations. Organics used in the process are fairly non-reactive based on available Material Specification Data Sheets.

For all three processes, separation of chemicals in storage and in use is required to safely operate the process. The FC process shows the least safety concern from that point of view. While the chemical reactivity is unfamiliar to tank farms, the use of nitric acid in processing waste is common and has been evaluated for other sites/facilities.

The individual evaluations of Reactive Chemicals for each of the Cs separation technologies can be found in CSSX-1.1.8, FC-1.1.8, and SRF-1.1.8.

\subsubsection{Criticality Safety}

From the nuclear criticality point of view, any process that is inherently sub-critical is preferred over a process that may have some potential to overcome sub-critical condition and requires criticality controls to maintain and monitor its sub-critical level. The IPS feed vector does not contain much transuranic material as shown by the staging feed composition. Our assessment looked at two major factors. These are: (1) Does the process have less than the minimal critical mass, and (2) Does the process alter the form and/or distribution of the TF waste. 
The CSSX, IX-sRF, and FC processes do not accumulate fissile material. Overall volume hold up in any of these systems is anticipated to be well below the minimum critical mass and approaches 15 grams (fissile exempt). Based on those concentrations, the three Cs separation processes are sub-critical under processing conditions. None of the three processes change the result of the TF-DSA evaluation.

The individual evaluations of Criticality Safety for each of the Cs separation technologies can be found in CSSX-1.2.1, FC-1.2.1, and SRF-1.2.1.

\subsubsection{Industrial Safety and Hygiene}

Industrial safety and hygiene was evaluated for each technology based on preliminary facility and equipment layouts and anticipated operation and maintenance requirements. For this definition a technology that results in fewer or less severe hazards is preferred over one that does not. These hazards include chemicals, noise, hot open surfaces, rotating equipment, access difficulties, elevated working surfaces, industrial sampling, ergonomic hazards, etc.

For the IX-sRF system, two large (57,500 gal) LAW product tanks, the feed receipt tank, cesium product tanks, spent resin accumulation tanks are located below grade. Access and egress for that equipment would be considered a confined space entry into a radiation zone. Similarly some pumps requiring routine maintenance in the confined radiation zone area will be located below grade. The process also provides for chemical $\left(\mathrm{NaOH}\right.$ and $\left.\mathrm{HNO}_{3}\right)$ storage on a grade-level area.

For the FC system, tanks and pumps are located in the below-grade area. Access and egress for that equipment would be considered a confined space entry into a radiation zone. The two crystallizers are approximately $30 \mathrm{ft}$ tall and span the below grade vault and above-grade structure. This equipment will require ladders and elevated walkways for access.

In the CSSX facility, CSSX equipment (centrifugal contactors, heat exchangers (2), and transfer pumps (7), and product vessels) is located below-grade area. Access and egress for that equipment and would be considered a confined space entry into a radiation zone. In addition, this technology also uses more chemicals (organic solvent, $\mathrm{NaOH} \mathrm{HNO}_{3}$ and $\mathrm{NaNO}_{2}$ ).

For all these three system facility layouts, not enough details are developed to identify ergonomic hazards at this time, and it is assumed that final design and layouts will minimize such hazards for maintenance and operation personnel.

The individual evaluations of Industrial Safety and Hygiene for each of the Cs separation technologies can be found CSSX-1.3.1, FC-1.3.1, and SRF-1.3.1.

\subsection{REGULATORY/STAKEHOLDER ACCEPTANCE}

\subsubsection{Achieve Tribal Nations/Stakeholder Acceptance}

From Tribal Nations/stakeholder acceptance point of view, the earlier a technology can be implemented the better it is. They are not necessarily interested in waiting for a perfect system to be implemented in the future, if the technology can remove or reduce the environmental risk as soon as possible. They want to know the extent of land that potentially would become contaminated ground. If a technology can achieve early treatment of waste with a minimum use of new land that can be contaminated, that technology will have a higher probability of being acceptable to the stakeholders. 
With respect of achieving early pretreatment both filtration technologies were judged to be capable of implementation in order to make early pretreatment viable, based on available technology maturation assessment information. No significant additional testing is required for ion exchange technology and industry standards are adequate for implementation. In contrast, some additional testing and modeling are required for both the CSSX and FC processes to verify their suitability to process Hanford LAW solutions.

Based on footprint calculations derived from the proposed IPS layouts for each technology, land use for the three cesium separation technology facilities are as follows:

- Ion-exchange facility $-4,032 \mathrm{ft}^{2}$ (with $\mathrm{RMF}$ ) and $4,610 \mathrm{ft}^{2}$ (with $\mathrm{CFF}$ )

- CSSX facility - 6,016 $\mathrm{ft}^{2}$ (with RMF) and 6,628 $\mathrm{ft}^{2}$ (with CFF)

- FC facility $-5,699 \mathrm{ft}^{2}$ (with RMF) and $5,963 \mathrm{ft}^{2}$ (with CFF)

These areas do not include support buildings, contingency expansion space, parking, etc. that would be required regardless of which technology is selected.

The individual evaluations of Achieve Tribal Nations/Stakeholder Acceptance for each of the Cs separation technologies can be found in CSSX-2.1.1, CSSX-2.1.2, FC-2.1.1, FC-2.1.2, SRF-2.1.1 and SRF-2.1.2.

\subsubsection{Achieve Regulator Acceptance}

From a regulatory perspective, the selected technology must be in compliance with all applicable regulations such as RCRA, CAA, NESHAPS, NEPA/SEPA, and DOE Orders, in order for it to be acceptable. The regulators also want to ensure that disposal system performance is maintained or improved, that secondary waste generation is minimized, and that it can be safely disposed. Any impact to other permitted facilities must also be looked at to ensure that no adverse impacts are found for those facilities.

It is important to understand whether the selected technology will meet current requirements and can be permitted. For National Environment Policy Act (NEPA), it is assumed that early LAW pretreatment with either of these filtration technologies will be covered by the Tank Closure and Waste Management (TC\&WM) Environmental Impact Statement (EIS) (expected completion in December 2009) and issuance of its Record of Decision (ROD) that is expected in January 2010. Three notices of construction will be required for the IPS and temporary storage tanks. The IPS and temporary tank storage units will constitute Resource Conservation and Recovery Act (RCRA) treatment, storage, and disposal (TSD) units and will require submittal of a Part B Permit Application pursuant to WAC 173-303-806, and Ecology issuance of a final status RCRA Part B permit prior to operation (WAC 173-303-840).

None of these three cesium separation technologies has been previously permitted for use at Hanford, but basic information exists to support the permit application process. For example, even though CSSX has not been tested on actual or simulated Hanford waste, it has been used at SRS. Additional Hanford waste specific testing and modeling work will be required. Similarly Fractional Crystallization is a common industrial process and was used at Hanford Site from 1974 through 1985. This process has been demonstrated at lab-scale using LAW solutions, and further demonstration tests are on-going for Hanford LAW. Ion exchange has been used to separate cesium from alkaline tank waste at Hanford Site. The spherical Resorcinol-Formaldehyde resin has undergone testing that demonstrates the minimum cesium decontamination factor can be achieved. Additional process development work is needed for the three technologies to support the permitting process. 
It is anticipated that the permitting process will take 32 months for any of the cesium separation technologies. No unusual difficulties are anticipated in obtaining various regulatory and stakeholder acceptance based on existing regulatory processes and requirements. RCRA, NEPA, SEPA, and $\mathrm{CAA} / \mathrm{NESHAPS}$ requirements can be satisfied.

In the past, state regulators have been amenable to expediting the permitting process to accelerate cleanup (e.g., the Integrated Disposal Facility). Unless the NEPA evaluation determines that one of the cesium separation technologies requires additional NEPA documentation, while neither of the others do, regulatory compliance does not appear to be a discriminator.

All three cesium removal processes generate secondary wastes. These secondary wastes include high efficiency particulate air (HEPA) filters, unknown quantity of PPEs, failed equipment, and chemical spill cleanup for disposal. These items can be disposed of using current practices for the current tank farm operations. HEPA filters and their upstream pre-filters are disposed of as LLW. Current mass balance flow sheets for cesium separation technologies do not identify COPC levels for the secondary wastes (e.g., spent ion exchange resin, FC liquid effluent to ETF, and spent CSSX organic solvents). The presence of technetium, iodine and chromium in the secondary waste could pose a challenge for disposal. The IPS facility will require appropriate regulated disposal approaches and can be accommodated in the plant design and operation procedures. Further investigation of secondary wastes for the three Cs separation technologies is required to determine COPCs, including technetium, iodine, and chromium.

Each Cs separation process also generates secondary wastes requiring special disposal alternatives. Fractional crystallization creates a large quantity of a liquid effluent stream that will be treated by ETF prior to its disposal. For processing of these eight tanks, a total of 7,256,143 L (1.9 M gallons, RPP-RPT37551 CH2M HILL 2008c, Table 6-22) of ETF stream is anticipated to be generated. For the FC process, the current waste acceptance criteria for the ETF will be met. Similarly the IX-sRF process will generate $4,535 \mathrm{~kg}(10,000 \mathrm{lbs}$, RPP-RPT-37551, CH2M HILL 2008c Table 6-11 thru 6-18) of used resin requiring a special disposal program. Caustic side solvent extraction process will generate approximately 680 to 1,475 L (180 to 390 gallons, RPP-RPT-37551, CH2M HILL 2008cTable 6-36) of liquid organic solvent for disposal per year. No secondary liquid waste requiring ETF processing is generated as a result of IX$\mathrm{sRF}$ or CSSX activities. Appropriate disposal program for these wastes will have to be developed.

Other impacts to the WTP Project's commissioning approach and to DST space management will have to be evaluated. Appropriate evaluations are needed to confirm that secondary waste generated from these cesium separation technologies meet applicable waste acceptance criteria. This includes waste going to ETF, discharges going to the Liquid Effluent Retention Facility, Cs-loaded waste streams going to the 242-A Evaporator for additional waste volume reduction, other streams going to DST for storage, or disposal of spent IX-sRF resin. There is insufficient information at this time to perform a more in-depth assessment of the secondary waste streams.

The individual evaluations of Achieve Regulator Acceptance for each of the Cs separation technologies can be found in CSSX-2.2.1 through CSSX-2.2.4, FC-2.2.1 through FC-2.2.4, and SRF-2.2.1 through SRF-2.2.4.

\subsection{TECHNICAL MATURITY/FLEXIBILITY}

Technical maturity and process flexibility and robustness of the selected technology are crucial factors in the successful completion of the IPS mission. A mature technology provides sufficient information for the design of the process equipment, services, and facility. Technical maturity is measured by assessing a Technology Readiness Level (TRL) for a given technology. As described in DOE 2008, a technology can 
receive a TRL rating between 0 and 9, with "9" representing the most matured technology. Once a TRL ha been determined for a given technology, a project will gradually mature that technology to the highest TRL number for its specific process application. Efforts (resources and time) required to mature the process and knowledge about the probability of success in attaining the fully matured technology will be different for each technology and should be determined. Therefore the TRL, as well as an estimate of the effort to mature each technology and its probability of success, are assessed in this section.

A flexible and robust technology for Hanford waste treatment projects will provide a large operation boundary so that it can be successfully applied to a variety of tank waste with differing characteristics and properties. Technology flexibility and robustness for IPS is measured with factors such as ability to process variety of feeds, ability to adjust process rate, flexibility to modify product, expandability, capability to recover from out of specification product, and applicability to other DOE complex projects.

The TRL workshop conducted by senior level management personnel has been described earlier. This workshop also considered cesium separation technologies in a manner described in Section 3.0. In the following sections, technical readiness level (Section 4.3.1) and process flexibility and robustness (Section 4.3.2) for the cesium separation technologies are summarized.

\subsubsection{Technology Readiness Level}

\subsubsection{TRL Number}

Two of the three cesium separation technologies (Fractional Crystallization and Ion Exchange using spherical Resorcinol-Formaldehyde resin) were assessed for technology readiness in 2007 (DOE 2007) and were assigned a TRL. In that assessment, which was based on test results using SST saltcake wastes and simulants, the FC technology was determined to have a TRL of Level 4. A TRL was attained if all questions at that level were answered as "Yes" with documented results. For Level 5 questions, FC technology was unable to answer "Yes" to many of the questions. During the more recent IPS workshop (DOE 2008), questions for Level 5 were reevaluated for the FC technology to assess whether the TRL had changed. The FC technology was found to have answered most Level 5 questions "Yes"; only two criteria responses were negative. The two remaining questions cover availability of plant-size components and establishment of requirements for technology verification. Since these two criteria were not satisfied with documented references, the FC technology still maintains TRL of Level 4. However, it should be noted that the feeds for IPS are primarily DST supernate wastes, rather than SST saltcake wastes.

The IX-sRF technology was determined to have a TRL of 3 in DOE/ORP-2007-01. During the IPS workshop questions for Level 4 were reevaluated to assess the Technology Readiness Level for the IXsRF technology (DOE 2008). Participants in the IPS workshop determined that there were still sufficient negative responses to the queries associated with TRL-4 such as to maintain the TRL of Level 3 for the IX-sRF technology. Specifically four questions could not be responded positively without documented references. These four questions cover science and technology exit criteria, draft conceptual design, formal risk management program, and functional work breakdown structure.

The CSSX technology was not evaluated in the Technology Readiness Assessment performed by DOE in 2007. To remain consistent with the other two TRL evaluations for the FC and IX-sRF technologies, the same questions were used for the CSSX technology in the IPS workshop. During the IPS workshop, the evaluation team concluded that there were valid negative responses to the criteria queries for Level 4 to conclude that a TRL of Level 3 was appropriate for the CSSX technology. The CSSX technology assessment identified five criteria lacking enough documented support to reach an acceptable "Yes" response. These five criteria covers items such as full identification of cross technology issues requiring 
preliminary hazard evaluation, science and technology exit criteria, draft conceptual design, functional work breakdown structure by user, and simulant developments covering the range of the waste properties. For some of these five criteria work is on-going, but it has not been completed and documented. Once that work is successfully completed and appropriately documented, a TRL of Level 4 will be attained.

The individual evaluations of TRL Number for each of the Cs separation technologies can be found in CSSX-3.1.1, FC-3.1.1 and SRF-3.1.1.

\subsubsection{Effort to Mature Technology (Cost and Schedule)}

A ROM cost and schedule was estimated for developing the candidate cesium separation technologies to attain a TRL of Level 6, thereby allowing transition into final design (consistent with DOE/ORP-200701). The estimate included only testing for technology maturity, i.e., development and qualification testing. Such activities as factory and construction acceptance and operational testing were not included in this estimate; these three project testing activities and all other project costs are covered by other assessments. This is a ROM estimate for comparison purposes only and final estimates will be documented in the Technical Maturation Plan. Table 4-3 presents the results of this effort.

Table 4-3 - Effort to Mature Solids Separation Technologies Comparison

\begin{tabular}{|c|c|c|}
\hline Technology & $\begin{array}{c}\text { Rough order-of-magnitude cost } \\
\text { (million dollars) }\end{array}$ & $\begin{array}{c}\text { Rough order-of-magnitude } \\
\text { schedule (months) }\end{array}$ \\
\hline \hline FC & 3.0 & 30 \\
\hline CSSX & 4.5 & 36 \\
\hline IX-sRF & 3.5 & 36 \\
\hline
\end{tabular}

The individual evaluations of Effort to Mature Technology (Cost and Schedule) for each of the Cs separation technologies can be found in CSSX-3.1.2, FC-3.1.2, and SRF-3.1.2.

\subsubsection{Probability of Success}

A subjective analysis of risk associated with the candidate cesium separation technologies was performed. Major risks were identified and evaluated for impacts to cost and schedule, resulting in the following ranking schema:

- High Probability of Success: Worst case risk is Medium

- Medium Probability of Success: Worst case risk is High

- Low Probability of Success: Worst case risk is Very High

Based on the analysis both FC and IX-sRF were judged to have a high probability of success. A medium probability of success was assigned to the CSSX. The individual evaluations of Probability of Success for each of the Cs separation technologies can be found in CSSX-3.1.3, FC-3.1.3, and SRF-3.1.3.

\subsubsection{Process Flexibility and Robustness}

\subsubsection{Ability to Process Variety of Feeds}

From the overall Hanford mission perspective, it is typically advantageous to select a technology that is sufficiently flexible to expand its application to various tank farm waste feed material, even though they are not currently included in the IPS project scope. This is a subjective evaluation based on existing 
configuration information, past test results, and an understanding of unit operations. Critical input stream characteristics include:

- Chemistry (composition, concentration, etc.)

- Physical properties (temperature, density, viscosity, etc.)

With respect to a waste stream's chemical properties, Fractional Crystallization is highly sensitive to analytes in the waste mixture that have similar solubility curves to sodium compounds. Envelope testing of analytes to determine the impact on proposed design and operation (CH2M HILL 2008a) has concluded that while some compositions impact operation there is no feed composition likely to be derived from salt cake dissolution that would cause the $\mathrm{FC}$ process to not meet performance requirements. The feed envelope study also concluded that there were no lower concentration limits for the process for the analyzed chemicals while an upper limit feed envelope was defined.

With respect to a waste stream's physical characteristics, both the crystallization process and centrifuge operation are sensitive to a variety of physical parameters affecting physical stream characteristics and behavior, including: crystallizer pressure, temperature, and feed rate, and centrifuge rotation rate, timing, and feed rate. These parameters are generally well-known and managed by standard design controls.

With respect to a waste stream's chemical properties, the CSSX process extraction efficiency is sensitive primarily to potassium, based upon modeling and testing (ORNL 2008). The 8 candidate IPS tanks have high potassium concentrations. This interference is mitigated by additional contactors; different solvents have also been proposed to reduce this sensitivity. Generally, extraction is also sensitive to solvent stability. Conversely, Oak Ridge testing of various simulants found that despite the variation of the concentrations of cesium, potassium, nitrate, and hydroxide, the performance of the scrub and strip stages was essentially the same from simulant to simulant.

With respect to a waste stream's physical characteristics, extraction efficiency and/or unit operation are sensitive to multiple factors affecting stream physical properties: contactor rotational speed, flow rates, general hydraulic performance, and stream temperatures (Parsons 2008).

Extensive modeling and testing of Savannah River, Hanford, and IPS waste streams have been conducted to manage IX sensitivities for various process feeds. With respect to a waste stream's chemical properties, these studies have found that cesium separation is highly sensitive to the type of IX resin. Significant evaluation of the selected resin, spherical Resorcinol-Formaldehyde (sRF), has been conducted to demonstrate its potential for meeting Cs removal rates and support IPS separation goals. Initial modeling for Hanford wastes has been conducted on the sRF resin, to replace the previous resin baseline of SuperLig ${ }^{\circledR} 644$ (Westinghouse 2004), which also compared model data with past PNNL resin tests. Additional modeling (Washington 2008) has been conducted to establish improved cesium adsorption isotherms for the 8 IPS candidate feed batches.

Cs capacity varies significantly with $\mathrm{pH}$ and is impacted by other cation concentrations ( $\mathrm{K}$ and $\mathrm{Na}$ ). However, rational selectivity coefficients for RF resin are consistently lower than the corresponding value for SuperLig ${ }^{\circledR} 644$ (Westinghouse 2004).

With respect to a waste stream's physical characteristics, modeling has evaluated sensitivity to flow rate, temperature, and column size (Washington 2008), with results for RF resin indicating trade-offs in overall processing timing from feed rate variation, better adsorption capacity at cooler temperatures, and insensitivity from column geometry. Pilot scale testing has been conducted to evaluate pressure drop and flow/adsorption performance (Washington 2006). 
The individual evaluations of Ability to Process Variety of Feeds for each of the Cs separation technologies can be found in CSSX-3.2.1, FC-3.2.1, and SRF-3.2.1.

\subsubsection{Ability to Adjust Process Rates}

A subjective evaluation was conducted on the ability of candidate cesium separation technologies to adjust the input feed rate while maintaining a stable operation. This evaluation was based upon existing configuration information, past test results, and understanding of the unit operations. The technology was categorized as follows:

- High Process Rate Flexibility = Can adjust process feed rate across a wide range, while having minimal effect on operation,

- Medium Process Rate Flexibility = Can only slightly adjust feed rates while maintaining stable operation,

- Low Process Rate Flexibility = Slight feed rate adjustment will have high probability of process upsets and require significant effort to recover technology stability.

Fractional Crystallization was judged to have High Process Rate Flexibility, while both CSSX and IXsRF were rated as having Medium Process Rate Flexibility. The individual evaluations of Ability to Adjust Process Rates for each of the Cs separation technologies can be found in CSSX-3.2.2, FC-3.2.2, and SRF-3.2.2.

\subsubsection{Flexibility to modify product}

A subjective evaluation was conducted on the candidate cesium separation technology's process flexibility and robustness. The following binning is arbitrarily defined to establish flexibility based upon degrees of freedom of technology choices and process control parameters.

- High product flexibility $=>12$ degrees of freedom.

- Medium product flexibility $=<12$ and $>5$ degrees of freedom

- Low product flexibility $=<5$ degree of freedom

This evaluation judged FC and to have high product flexibility. Both CSSX and IX-sRF were judged to exhibit medium product flexibility. The individual evaluations of Flexibility to Modify Product for each of the Cs separation technologies can be found in CSSX-3.2.3, FC-3.2.3, and SRF-3.2.3.

\subsubsection{Ability to Expand}

This is a subjective evaluation of current capability based upon existing configuration information, past test results, and understanding of the unit operations. This effort supports determination of the overall Technology Readiness Level. Critical expansion characteristics include:

- Ability of existing design to handle additional feed volumes

- Ability for installation of addition separation unit operations into existing footprint

Evaluation assumes this flexibility is based upon additional volume/mass only with all other waste conditions remaining constant. The FC crystallizer is sized ( 6200 gallons) to provide an 8-hour residence time at the maximum centrifuge rate. The centrifuge rate of $15 \mathrm{gpm}$ is based upon maximum steady-state flow from the crystallizer. Any capacity increase would require significant equipment size increases as well as a significant footprint modification. 
The number of CSSX contactors (43 overall for extracting, stripping, scrubbing, and washing) is based upon a worst case feed application for cesium decontamination. Some flow rate increases are possible, but the technology is highly sensitive to balanced feed and organic flow rates. Any volume processing increases would require resizing of the contactors or installation of parallel processing lines. Size increases would have a limitation based on contact kinetics, but would only require a moderate footprint increase. Installation of a parallel line (most practical for expansion sizing) would require a significant footprint increase.

Ion exchange column for IX-sRF is sized based upon a nominal design rate of $192 \mathrm{~kg} \mathrm{Na} / \mathrm{hr}$. No excess capacity is projected from this design, but could be incorporated into the IPS design if desired. Capacity increases would require moderate equipment size increases as well as a footprint modification.

The individual evaluations of Ability to Expand for each of the Cs separation technologies can be found in CSSX-3.2.4, FC-3.2.4, and SRF-3.2.4.

\subsubsection{Recover from Out-of-Specification Product}

This is a subjective analysis based upon the current process flow sheet, without specific systems assumed for recycle and storage to address out of specification product. Flexibility is defined by the following binning:

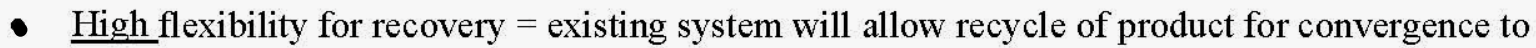
proper specifications without additional hardware systems (assumes minimal software changes).

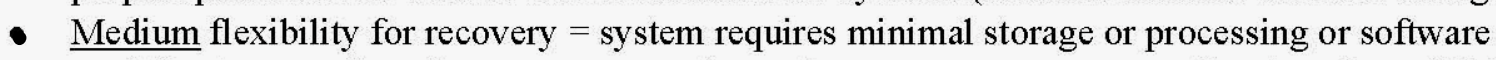
modifications to allow for convergence of recycle streams to proper specifications (e.g., \$1M $\$ 5 \mathrm{M})$

- Low flexibility for recovery = system requires significant storage or processing or software modifications to allow convergence of recycle streams to proper specifications. (e.g., $\$ 5 \mathrm{M}$ $\$ 20 \mathrm{M})$

- Very Low flexibility for recovery = final product can no longer be further processed to improve specification; out-of specification material must be segregated for special disposal handling or blending for other treatment processes.

The current FC design employs recycle line from second stage crystallizer into first stage. System chemistry and configuration allow for the installation of recycle piping from final product tanks into crystallizer feed tank. System would require minor tank storage changes to handle out of specification product while recycling.

The current CSSX design does not employ a recycle system to address product with insufficient cesium decontamination. However, system chemistry and configuration allow for the installation of recycle piping from final product tanks into contactor feed tank. Rough order of estimate magnitude cost is $<\$ 5 \mathrm{M}$ using existing tanks and pumps.

The current IX-sRF system design employs recycle lines back to the lead and polishing columns. This technology allows for simple reprocessing of any recycle stream to improve Cs decontamination, with added flexibility to use either lead or polishing column. The IX-sRF system would require minor tank storage changes to handle out of specification product while recycling.

The three Cs separation technologies are all rated as Medium flexibility for recovery. The individual evaluations of Recover from Out-of-Specification Product for each of the Cs separation technologies can be found in CSSX-3.2.5, FC-3.2.5, and SRF-3.2.5. 


\subsubsection{Technology Applicability to Other DOE Complex Projects}

In a subjective analysis, an attempt was made to define cesium separation technology interface in relationship to other pretreatment activities occurring across the DOE complex. Applicability standings for these subjective assessments are defined earlier in Section 3.3.2.

Based on the definitions for applicability previously outlined in Section 3.3.2.6, the technical applicability of Fractional Crystallization is rated "No". This technology is under investigation at Hanford only for potential pretreatment of tank wastes. It has not been deployed nor planned for final deployment in fullscale hot operations at any other DOE complex projects.

Conversely the IX-sRF technology's technical applicability for both the IX-sRF and CSSX technologies are rated as "High". The IX-sRF technology with specified ion exchange resin is being deployed in the Hanford WTP. The CSSX technology is deployed at the SRS Modular CSSX Unit for full-scale hot operations for SRS tank wastes.

The individual evaluations of Technology Applicability to Other DOE Complex Projects for each of the Cs separation technologies can be found in CSSX-3.2.6, FC-3.2.6, and SRF-3.2.6.

\subsection{OPERABILITY AND MAINTAINABILITY}

The IPS facility will require significant operation and maintenance (O\&M) support. These O\&M activities play an important role in the success of the mission. To balance the technology selection, various operation and maintenance related measures were evaluated. These measures include, ease of process control and operation, ALARA principles, reliability of process equipment, ease and frequency of maintenance, ease of process implementation, and liquid/solid secondary waste. All three cesium separation technologies were evaluated against these measures and assessments are provided below.

\subsubsection{Ease of process control and operation}

As discussed in Section 3.4, many factors affect ease of process control and operation. For radioactive operation, easier process control and operation is considered to result in the effective operation with a minimum of errors and accidents. Items such as system complexity, number of chemicals, process samplings needs, operating margin and flexibility, standby conditions, etc. are various factors that measure "ease of process control and operation" and must be reviewed. At Hanford, where interfaces with the tank farms are very crucial, complexity of transfers to, from, and within the tank farm system is also an important consideration.

\subsubsection{Minimize number and frequency of surveillances}

Various process parameter measurements and recordings, as well as leak detection are required to properly monitor operation of radioactive processes. The process parameters include tank levels, pump amperage, temperatures, pressures, etc. Preliminary review of cesium separation flow sheets identified at least 58 process parameters and 11 sump leak detectors requiring measurements and recording during routine equipment operation for the FC process. For the CSSX process 38 process parameters, 20 sump leak detectors and 98 other equipment-related data points require measurement and recording during routine operation. These other equipment data points are related primarily to the 43 centrifugal contactors used in the process. For the IX-sRF process, 26 process parameters and 18 sump leak detectors have been identified. Of course, these numbers will be adjusted as design is further developed. Many parameters can be measured and monitored through the process DCS. In our assessment these parameters are comparable to a similar type and size of process facility. 
The total list of parameters is provided with the assessment summary form for each technology in CSSX4.1.1, FC-4.1.1, and SRF-4.1.1.

\subsubsection{Minimize number of people to operate}

The number of personnel required to operate and monitor the process is yet another indicator of the complexity and sensitivity of the process to potential process upsets. Preliminary assessments show about an equal number of operating personnel will be required for each process. The personnel requirement for FC and IX-sRF system is 10 people and 11 people for CSSX system. Types of personnel include control room operators, sampling system operators, health physics technician, instrument technician, electrician, engineer, and supervisor.

A preliminary list of required personnel is attached with the respective assessment summary form for each Cs separation technology in CSSX-4.1.2, FC-4.1.2, and SRF-4.1.2.

\subsubsection{Ease of startup and shutdown}

Once initiated, IPS process operation may require going to either a standby or a temporary shutdown due to several reasons. Ease of startup and shut down is important for the plant operation. Startup and shutdown process for IX-sRF system seems to be relatively simple. Starting and stopping the waste feed to the ion exchange columns starts or shuts down the process respectively.

Start up of FC system is a two-stage process where first the cooling water system, vacuum and off gas systems are made operational before the preheating of the re-boilers and crystallizers is started.

Shutdown of the FC system may require dilution of the salt solutions in the re-boilers and crystallizers to prevent plugging with solids that form at lower temperatures. The startup and shutdown of FC can be performed independently depending on the volumes and concentration of the materials in first and second stage crystallizers. In FC, the two-stage process requires extensive monitoring in approach to equilibrium condition.

The CSSX process is normally started up in the reverse order of process operation, i.e., the wash and scrubbing contactors are started up first, followed by the stripping contactors, and finally the extraction contactors and waste feed pump until proper equilibrium is reached. The shutdown is achieved by stopping the feed stream, but extraction contactors should be operated until they are stripped of all feed material. Due to equilibrium condition requirements start up and shutdown of CSSX system may be the most time consuming. Equilibrium conditions are more difficult to establish during the introduction of the feed stream to extraction contactors.

The individual evaluations of Ease of Startup and Shutdown for each of the Cs separation technologies can be found in CSSX-4.1.3, FC-4.1.3, and SRF-4.1.3.

\subsubsection{System complexity}

The complexity of process system can be inferred by the number significant components, support systems and controls that are required to operate. A preliminary list of various active, passive and other components of each technology is provided below in Table 4-4.

In general, each tank will require instrumentation to measure level, specific gravity, temperature and differential pressure. Each cell in the facility will require a leak detector, alarm system, and/or sump pump to remove collected liquid. All these instrumentation and detection and measurement equipment will have to be connected to the facility DCS system for monitoring and controlling. Overall review of these components shows that complexity order from high to low is CSSX, FC and IX-sRF system. 
The individual evaluations of System Complexity for each of the Cs separation technologies can be found in CSSX-4.1.4, FC-4.1.4, and SRF-4.1.4.

Table 4-4 - Operation Components for Cesium Separation Processes

\begin{tabular}{|c|c|c|c|}
\hline Item & FC & CSSX & IX-sRF \\
\hline \multicolumn{4}{|l|}{ Active Components } \\
\hline Pumps & 15 & 51 (with contactors) & 13 \\
\hline Centrifuges & 2 & & \\
\hline Centrifuge wash systems & 2 & & \\
\hline Cooling water system & 2 & 1 & 1 \\
\hline \multicolumn{4}{|l|}{ Passive Components } \\
\hline Flow control Valves & 16 & 8 & 5 \\
\hline Re-boilers & 2 & & \\
\hline Crystallizers & 2 & & \\
\hline Tanks & 19 & 23 & 18 \\
\hline Condensers & 6 & & \\
\hline Steam jets & 2 & & \\
\hline Vault ventilation system & 1 & 1 & 1 \\
\hline Air handling unit & 1 & 1 & 1 \\
\hline Process off-gas ventilation unit & 1 & 1 & 1 \\
\hline Resin columns & & & 2 \\
\hline In tank mixers & & & 3 \\
\hline
\end{tabular}

\subsubsection{Need for number of chemicals}

Number and type of chemicals required to operate a chemical process play an important role in the operation of processing facilities such as these. The lower the number of these chemicals and the less complex their handling, the better it is for the process operations. Chemical usage is minimal in the FC system (no added chemicals). The IX-sRF system uses only 3 basic chemicals $\left(\mathrm{NaOH}, \mathrm{NaNO}_{2}\right.$ and $\mathrm{HNO}_{3}$ ). On the other hand, the CSSX process uses variety of chemicals, including specialty solvent mixture, $\mathrm{HNO}_{3}, \mathrm{NaOH}$, and $\mathrm{NaNO}_{2}$. The specialty solvent mixture includes a diluent, an extractant, and a modifier. A total of seven different chemicals are required to operate the CSSX process.

The individual evaluations of Need for Number of Chemicals for each of the Cs separation technologies can be found in CSSX-4.1.5, FC-4.1.5, and SRF-4.1.5.

\subsubsection{Process and regulatory samples}

For the FC process, a total of five (5) process samples may be required per day. These samples include cesium product, LAW products, and vacuum jet condensates. Liquid effluent sample may be required prior to sending the condensate stream to ETF.

For the CSSX process the cesium product stream is to be sampled on a daily basis during routine operation for process control purposes. In addition, aqueous and organic streams from the final extraction contactors are to be sampled on a daily basis. Solvent holding tanks may be sampled at least on a weekly basis. The fresh scrub and wash solutions should be sampled on a batch basis. Spent solvent will require planned sampling for regulatory requirements. 
For the IX-sRF process, cesium product samples will be required on a daily basis to assure process conformance. Aqueous makeup of nitric acid and sodium hydroxide solutions are to be sampled on a batch basis. Spent resin samples will be required on a batch basis to assure compliance with on-site disposal requirements.

The individual evaluations of Process and Regulatory Samples for each of the Cs separation technologies can be found in CSSX-4.1.6, FC-4.1.6, and SRF-4.1.6.

\subsubsection{Batch verses continuous operation}

By nature of the process itself, CSSX should be operated as a continuous process. Intermittent shut downs and re-startups should be avoided. In FC system, evaporators can be operated on a continuous basis, but centrifugation and product washing are done as a batch operation. IX-sRF system will be operated on a batch basis due to the nature of the elution and regeneration operating cycle.

The individual evaluations of Batch verses Continuous Operation for each of the Cs separation technologies can be found in CSSX-4.1.7, FC-4.1.7, and SRF-4.1.7.

\subsubsection{Ease of entry and exit from standby}

Any process may require a temporary shutdown for a variety of reasons. The selected process should be able to easily enter into and exit from a standby condition. Restart from a temporary shutdown or standby condition has a minor impact on the FC system as long as shutdown conditions exist for a short time only. Requirements to restart are to reestablish or verify adequate vacuum, restart of re-boilers/crystallizers, reestablish recirculation, and turn on feed pumps. The dissolver and centrifuges can be restarted independent of the re-boiler and crystallizers.

The restart of the CSSX system from the temporary shutdown is no different than the restart from a major shutdown and cannot be done easily in a short time period. There are significant operational and production impacts if a CSSX system is required to enter into a standby mode. If required, a temporary shutdown of the CSSX would include shutting of the feed pump and continued operation of the contactors until cesium is stripped from the solvent.

Temporary shutdown is achieved most easily for IX-sRF process by shutting off the waste feed pump. The restart is accomplished by reestablishing waste feed flow. Ion exchange column operation is not impacted due to shutdown conditions. If shutdown lasts for several days, the resin column should be rinsed, eluted, and regenerated to protect the resin from radiation and chemical degradation.

The individual evaluations of Ease of Entry and Exit from Standby for each of the Cs separation technologies can be found in CSSX-4.1.8, FC-4.1.8, and SRF-4.1.8.

\subsubsection{Wide operating margin}

In this evaluation, range of waste feed chemicals and solids that systems can efficiently process were evaluated. While the FC technology appears to be able to process a wide range of waste compositions, the development work to date has focused on SST salt cake, rather than the DST wastes included in the 8 IPS candidate batches. Waste feeds containing high phosphates may require dilution of the Cs-loaded stream prior to transfer to the DST system in order to prevent precipitation of the phosphates, potentially leading to plugging of waste transfer lines. The degree to which the FC process can effectively remove sulfates from the Cs-depleted waste stream being transferred to WTP will also impact the number of ILAW containers produced. 
The CSSX technology can accommodate most soluble tank waste chemicals. However, it requires that the waste feed sodium concentration first be adjusted to approximately $6 \underline{\mathrm{M}} \mathrm{Na}$ to prevent aluminum precipitation. Cesium removal is also somewhat dependent on the potassium concentration in the waste feed stream. If the potassium concentration is high, the solvent extraction may have to operate with a higher organic-to-aqueous flow ratio, thereby creating a more dilute Cs-loaded stream being returned to the DST system.

The IX-sRF system requires adjustment of the waste feed sodium concentration (to approximately $6 \underline{\mathrm{M}}$ $\mathrm{Na}$ ) to prevent aluminum precipitation and to reduce viscosity of the waste feeds stream to prevent excessive pressure drop across the column during operation. The system must be operated with a filtered feed stream to prevent resin column plugging problems.

The individual evaluations of Wide Operating Margin for each of the Cs separation technologies can be found in CSSX-4.1.9, FC-4.1.9, and SRF-4.1.9.

\subsubsection{Complexity of transfers to, from and within tank farms}

None of these three cesium separation processes introduce any complex transfers to, from, or within the tank farm. The required transfers are routine and do not invoke any special requirements. Frequency of transfers may be different for each process.

In order to recover the optimum volume of DST space, the CSSX process will require significant volume reduction of the Cs-loaded stream at the 242-A Evaporator. This increases the total number of waste transfers and complexity of DST space management logistics required to support the CSSX.

The individual evaluations of Complexity of Transfers to, from and within Tank Farms for each of the Cs separation technologies can be found in CSSX-4.1.9, FC-4.1.9, and SRF-4.1.9.

\subsubsection{ALARA}

For the operation of IPS, ALARA represents the one of the most critical operating principles. The facility design and processes must address ALARA principles for the handling of radioactive components as well as other chemicals, which can be hazardous or toxic and can be harmful to personnel and the environment. For handling components or chemicals, contact maintenance should be minimized to reduce personnel exposure and risk. In this section, operational impacts from cesium separation technologies for this definition are assessed. Facility related ALARA issues will be addressed during the engineering design phases.

The FC system has one waste feed pump, two centrifuges, and 15 process pumps that will require handson or contact maintenance. These components contain radiological and hazardous chemical materials. The process also has an undetermined number of process flow meters and flow control valves. This equipment will require hands-on maintenance. The process condensate system components could potentially contain some quantity of radioactive waste as the result of process upsets and it may require hands-on maintenance for pump, condenser or steam jet work.

In the CSSX facility, 51 process-related pumps and centrifugal contactors will require hands-on or contact maintenance for all repair and replacement activities, unless the IPS is equipped with an overhead crane, remote equipment connectors, and an inventory of spare parts. In addition, there are seven (7) chemical transfer pumps in the aqueous chemical make-up area requiring hands-on maintenance.

In the IX-sRF process, there are 13 pumps and two (2) ion exchange columns requiring hands-on or contact maintenance for all repair and maintenance activities. 
In all cases, the facility design can be adjusted to increase remote maintenance of highly contaminated components which will reduce contact maintenance activities. Special remote maintenance areas with handling equipment may help exposure to radiological and chemical hazards during contact maintenance. Facility ventilation system, air handling unit, and a process ventilation system also will require hands-on maintenance activities. The majority of the instrument lines, leak detectors, flow meters, and radiation detectors are potentially contaminated by tank waste constituents, but do not represent a significant hazard for routine calibration and operability checks. Their replacement would expose personnel to radiological and chemical hazards during hands-on activities.

The individual evaluations of ALARA for each of the Cs separation technologies can be found in CSSX4.2.1, FC-4.2.1, and SRF-4.2.1.

\subsubsection{Reliability}

Reliability of components and systems will play an important role in operation and maintenance of the IPS. Passive systems or systems with a fewer number of active components will contribute to better overall reliability. Also reliability of analogous system used in similar environments may provide a better indication of how it may perform within the IPS. These factors are assessed below for the three cesium separations technologies.

In the CSSX facility, sixty (60) major active components (pumps and centrifugal contactors) are identified. The nominal failure rate for the sixty components is once every 3-5 years. Solvent extraction with centrifugal contactors has not been performed at Hanford, but reliability data should become available with operation of the SRS's Modular CSSX Unit (MCU). A solvent extraction process was used in PUREX operations, but it was sufficiently different such that equipment and process reliability data are not applicable to IPS.

In the IX-sRF process, there are 14 process-related active components. The four pumps used for feed and product transfers have a failure history of once every 3-5 years. The 10 chemical pumps have a failure history of once every 6-8 years. Hanford site personnel have extensive ion exchange system operation experience. Though performance of $\mathrm{sRF}$ resin has not been demonstrated in actual operation, the ion exchange system reliability is enhanced by site operating experience.

The fifteen (15) pumps, two (2) centrifuges and 1or 2 cooling water skids are the major active components in the FC systems. The average operating life of waste feed pump is 5 years. Process transfer pumps should fails once per 6-9 years. From the standpoint of view of reliability in analogous systems, the successful operation of the 242-A and 242-S Evaporators provides a sound basis for the probable reliability of the FC system components.

The facility for each cesium separation technology has a vessel ventilation, building ventilation system, and air handling units with some active components. The individual evaluations of Reliability for each of the Cs separation technologies can be found in CSSX-4.3.1, FC-4.3.1, and SRF-4.3.1.

\subsubsection{Ease and frequency of maintenance}

Maintenance activities have an important role in the overall success of the IPS facility operation. Ease and reduced frequency of maintenance helps achieve cost effective operation and improves the plant's total operation efficiency. To assess this measure various items, such as support systems, preventative maintenance requirements, types of maintenance, and difficulty of maintenance with complex and specialized equipment are considered. For Hanford application, a minimal number of activities within the DST environment is preferred. 
Process-related support systems required by each cesium separation technology are identified. All three technologies require three standard services of water, cooling water, and air for instruments and valves. The FC will also require steam service for the heating of the re-boiler and for operating the vacuum jets in the off-gas system

Minimizing the number and frequency of preventive maintenance activities is important. Such processspecific activities are dependent on active and passive components, equipment such as, tanks, instrumentations, and valves. The following table provides a list of these items for each technology.

Table 4-5 - Components and Equipment items for Maintenance

\begin{tabular}{|l|l|l|l|}
\hline Item & \multicolumn{1}{|c|}{ FC } & \multicolumn{1}{c|}{ CSSX } & \multicolumn{1}{c|}{ IX-sRF } \\
\hline Active components & $\begin{array}{l}19 \text { (pumps, water skids, } \\
\text { centrifuges, building } \\
\text { ventilation) }\end{array}$ & $\begin{array}{l}\text { 61 (pumps, decanters, } \\
\text { contactors, water skids, } \\
\text { building ventilation) }\end{array}$ & $\begin{array}{l}\text { 16 (pumps, water } \\
\text { skids, building } \\
\text { ventilation) }\end{array}$ \\
\hline Passive components & $\begin{array}{l}12 \text { (HX, crystallizers, } \\
\text { re-boilers, filters) }\end{array}$ & 1 (HX) & 4 (HX, resin columns) \\
\hline Other equipment & 13 tanks/vessels & 15 tanks/vessels & 13 tank/vessel \\
\hline Instrumentations & Instrumentation TBD & Instrumentation TBD & Instrumentation TBD \\
\hline Components & $\begin{array}{l}\text { Piping components } \\
\text { (valves, etc.) }\end{array}$ & $\begin{array}{l}\text { Piping components } \\
\text { (valves, etc.) }\end{array}$ & $\begin{array}{l}\text { Piping components } \\
\text { (valves, etc.) }\end{array}$ \\
\hline
\end{tabular}

The CSSX and FC systems have a larger number of rotating components and, therefore, will require for more preventive maintenance (PM) activities and a higher number of zone entries for maintenance than the IX-sRF system. The centrifugal contactors in the CSSX systems are very specialized component and will require special attention for maintenance activities. The FC and IX-sRF systems use commercial equipment that is modified for operation in a radioactive environment. None of these three cesium separation technologies require direct entry into DST space.

The individual evaluations of Ease and Frequency of Maintenance for each of the Cs separation technologies can be found in CSSX-4.4.1, FC-4.4.1, and SRF-4.4.1.

\subsubsection{Ease of Implementation}

One would intuitively prefer that the selected technology be easily implemented. If technology implementation and operations are too complex, it may introduce human errors as well as require extensive training and retraining programs that will make it less cost effective. The three cesium separation technologies are assessed for ease of training, procedure implementation, and familiarity with similar procedures which were considered key elements in determining ease of implementation.

The FC system utilizes evaporator-type equipment similar to that used at Hanford's 242-A Evaporator. Tank Farm personnel are generally familiar with the operation and maintenance of evaporator components. The familiarity with portions of the FC system should reduce some of the training requirements. However, the FC system requires a more complex flow sheet and contains a significant number of new equipment items and operations that will increase the overall training requirements. The number and complexity of procedures for the FC system will be increased.

Solvent extraction processes have been used extensively in the past at the Hanford site. The CSSX system is currently in use at the Savannah River site. However, the CSSX system has a large number of new equipment items (centrifugal contactors) that will require an increased amount of operations and 
maintenance training and qualification. There will also be an increased number and complexity of procedures for the CSSX system.

Ion exchange processes have been used extensively in the past at the Hanford site. The IX-sRF system has the simplest flow sheet and the least number of active components. The number of procedures may increase based on the number of specific operations within the process, but the complexity of the procedures will be reduced. The training regimen should be at or below current requirements.

The individual evaluations of Ease of Implementation for each of the Cs separation technologies can be found in CSSX-4.5.1, FC-4.5.1, and SRF-4.5.1.

\subsubsection{Liquid/Solid Secondary Waste}

All of the processes generate some amount of secondary waste. It is important to ensure compliance with DOE Order 420.1B and to minimize operational impacts associated with hazardous (generated) waste handling.

DOE Order 420.1B provides the general aspects of safety to be included in DOE facilities, and covers Nuclear and Explosion safety design, Fire Protection, Criticality Safety, NPH Mitigation, and the System Engineer Program. Review of the order finds no features of these three cesium separation technologies that would not meet the order requirements and that the IPS design could easily accommodate the requirements with any of the candidate technologies

All cesium separation processes produce two major product streams, a Cs-depleted feed stream that will be transferred to the WTP for ILAW and a Cs-loaded stream that will be transferred to the DST system for future $\mathrm{HLW}$ feed. The FC process generates process condensate as a secondary waste stream, which is transferred to ETF for further treatment and disposal. No operational impacts are anticipated for the transfer to ETF as long as the IPS facility design includes sufficient storage capacity.

The CSSX process generates a degraded organic solvent stream as a secondary waste stream that must be sampled, treated with absorbents, and packaged for on-site disposal. The degraded organic handling should not create any unusual operational impacts. The IX-sRF process generates spent ion exchange resins as a secondary waste stream that will require sampling and packaging for on-site disposal. Resin change outs will require handling equipment that must be designed into the facility. The resin handling activities are not anticipated to create any unusual operational impacts.

The individual evaluations of Liquid/Solid Secondary Waste for each of the Cs separation technologies can be found in CSSX-4.6.1, FC-4.6.1, and SRF-4.6.1.

\subsection{PROGRAMMATIC ASPECTS}

Several programmatic considerations have the potential to impact the selection of the technologies required for the Interim Pretreatment System. In some cases, these factors may become predominant and require a more complete and careful evaluation. From a programmatic perspective, the major factors for the IPS technology evaluations are: cost, implementation schedule, DST space management, impacts to WTP/supplemental treatment, impacts to other site facilities, and availability of needed resources and material. In the following sections, these factors are assessed for the three cesium separation technologies, FC, CSSX and IX-sRF. 


\subsubsection{Cost Impacts}

Tank Farms waste is required to undergo both entrained solids filtration and Cs separation prior to acceptance at the WTP LAW facility. Since there is always a pairing of a solids filtration technology with a Cs separation process, the one with the higher throughput requirement drives the overall processing capacity, and hence the capital and consumables costs. Cost estimates for a technology independent of its specific pairing is not practical due to this capacity dependence. Consequently, six separate cost estimates have been developed for the six possible pairings between the two solids separation technologies of rotary micro-filtration and cross-flow filtration with the three Cs extraction technologies of ion exchange using spherical Resorcinol-Formaldehyde resin, caustic-side solvent extraction, and fractional crystallization.

For the purpose of technology comparisons, the costs for ancillary items such as control trailer, transformer, switchgear, motor control center, power transmission lines, water supply lines, and sanitary waste removal have not been included since they are assumed to be similar for each option. Capital costs include engineering design, project management, construction management, and construction costs.

Construction costs are based on the quantities developed from the process layouts and equipment lists developed in RPP-RPT-37551 (CH2M HILL 2008c). The life-cycle costs include operating and maintenance costs for the 5-year IPS and D\&D costs, in addition to capital costs. The operation and maintenance costs are based on $24 / 7$ operation over a five-year period. The major chemical consumables and utility costs are likewise based on the quantities developed from the process layouts and equipment lists developed in RPP-RPT-37551 (CH2M HILL 2008c). The cost profiles were developed using estimator experience and judgment. There was no specific attempt to optimize the cost profiles in the six estimates. The project schedule was used for escalating costs.

The estimates represent a Class 4 level of project definition as defined by the Association for the Advancement of Cost Engineering (AACE) International and are for comparative purposes only. A Class 4 estimate has an expected accuracy range from a minus $30 \%$ to a plus $50 \%$.

Fractional crystallization is the only Cs separation technology requiring a significant amount of process steam. Estimated boiler and fuel costs are unique to the two FC pairings. The capacity of the fuel oil heated steam supply system estimated for the two options is driven by the high throughput requirement of one of the eight candidate waste streams. It is acknowledged that the boiler will operate and consume fuel at this maximum capacity only $1 / 8$ of the total operating time. The other $7 / 8$ of the operating time the boiler will service a reduced process throughput at a ratio of $9 / 17$. Consequently, average annual fuel consumption has been incorporated into the estimate.

The IX-sRF technology for Cs separation when paired with the CFF technology for entrained solids filtration results in the lowest estimates for both project capital cost and life-cycle cost. The FC technology for Cs separation when paired with the RMF technology for entrained solids filtration results in the highest estimates for both the project capital cost and life-cycle cost.

Figures 4.1 and 4.2 summarize the capital cost and life-cycle cost estimates for each pairing of technologies, respectively, while Figures 4.3 through 4.8 provide cost profiles over the life-cycle for each of the 6 technology pairings. Detailed estimates can be found in Attachment F and the Basis of Estimate in Attachment I of RPP-RPT-37741 (CH2M HILL 2008d). 


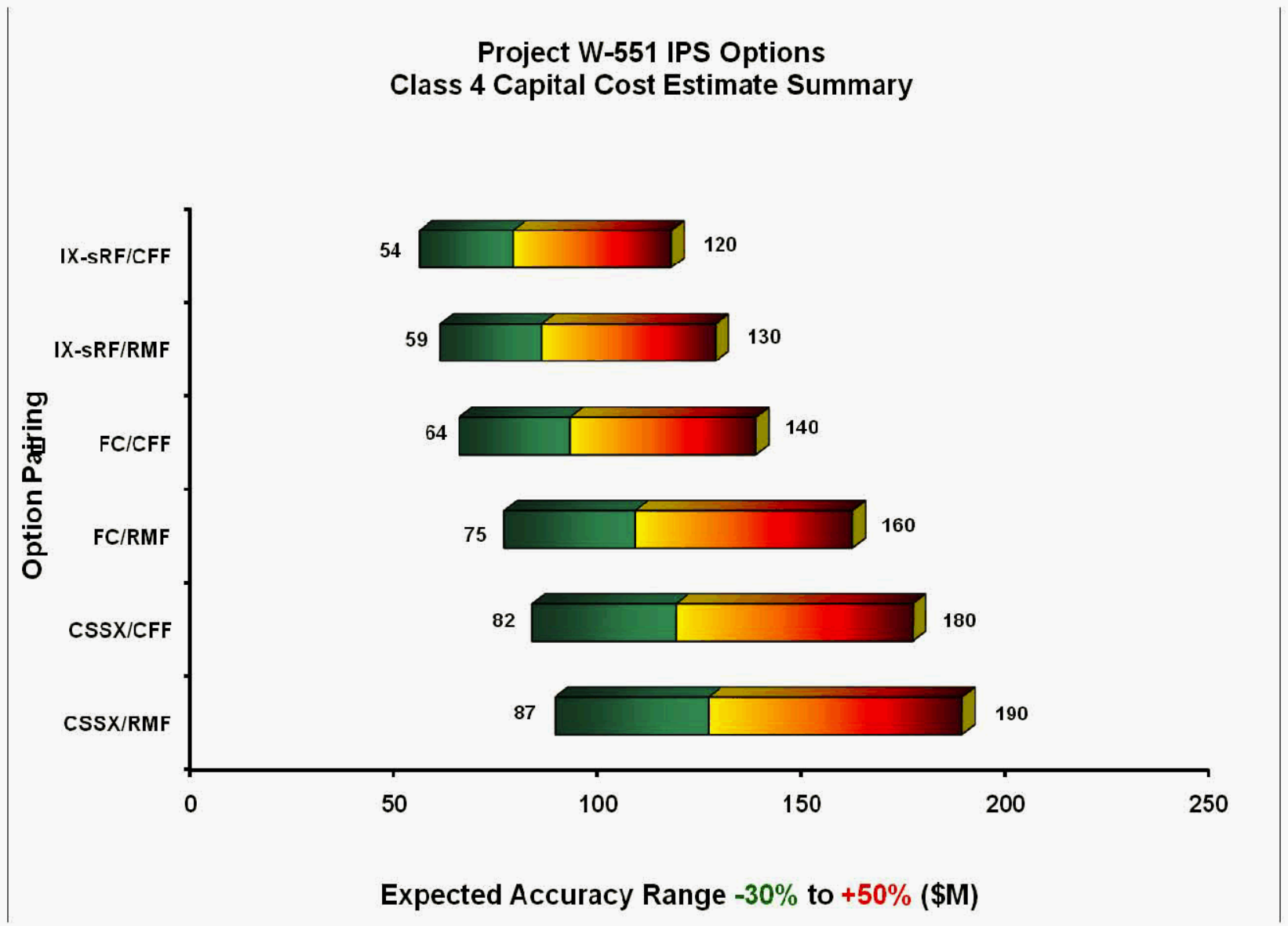

Figure 4-1 - Class 4 Capital Cost Estimate Summary for Project W-551 IPS Options 


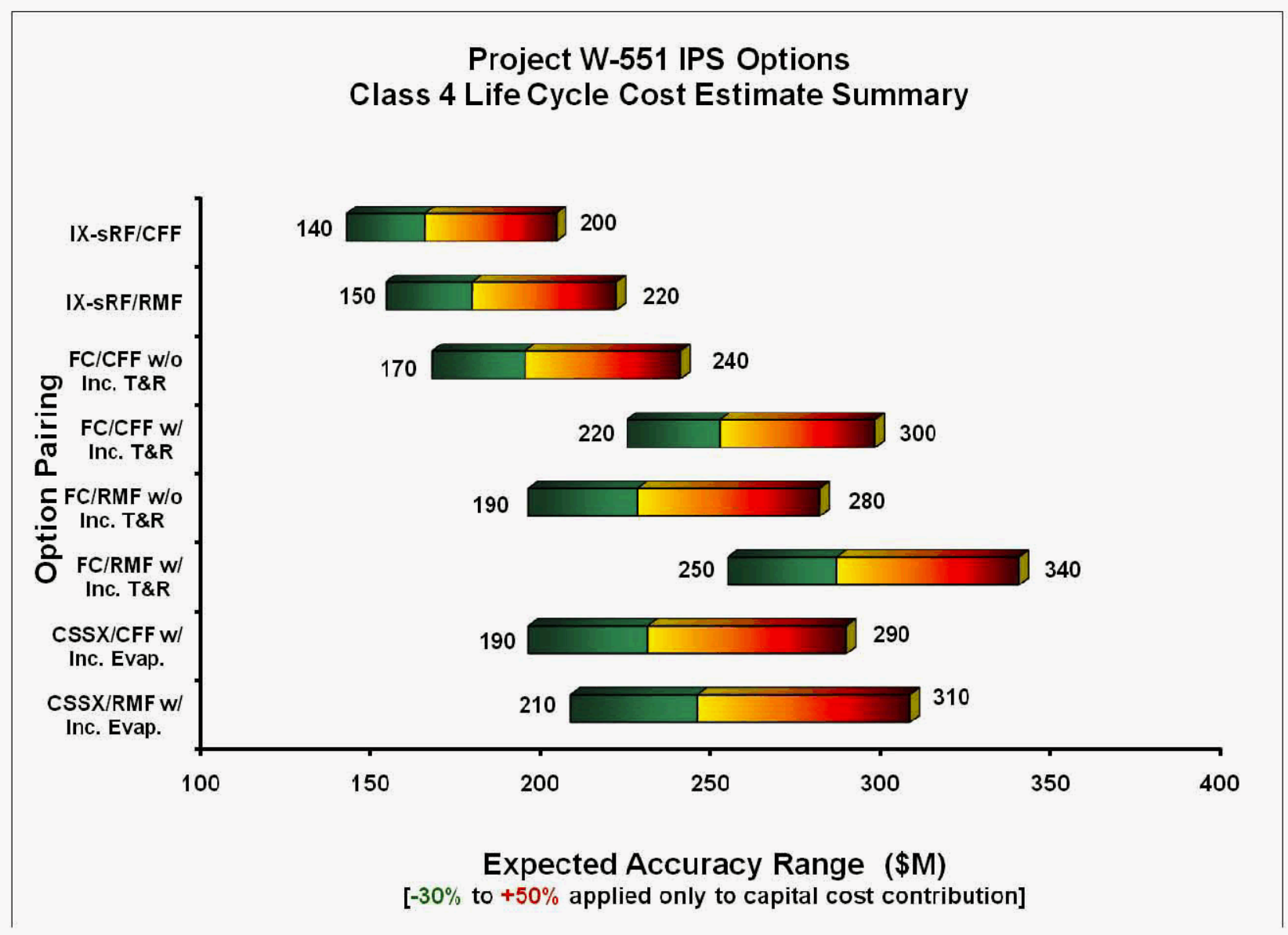

Figure 4-2 - Class 4 Life-Cycle Cost Estimate Summary for Project W-551 IPS Options 
Project W-551 Base Life Cycle Cost Profile

Ion Exchange using spherical Resorcinol-Formaldehyde resin \& Crossflow Filtration (no expected accuracy range applied)

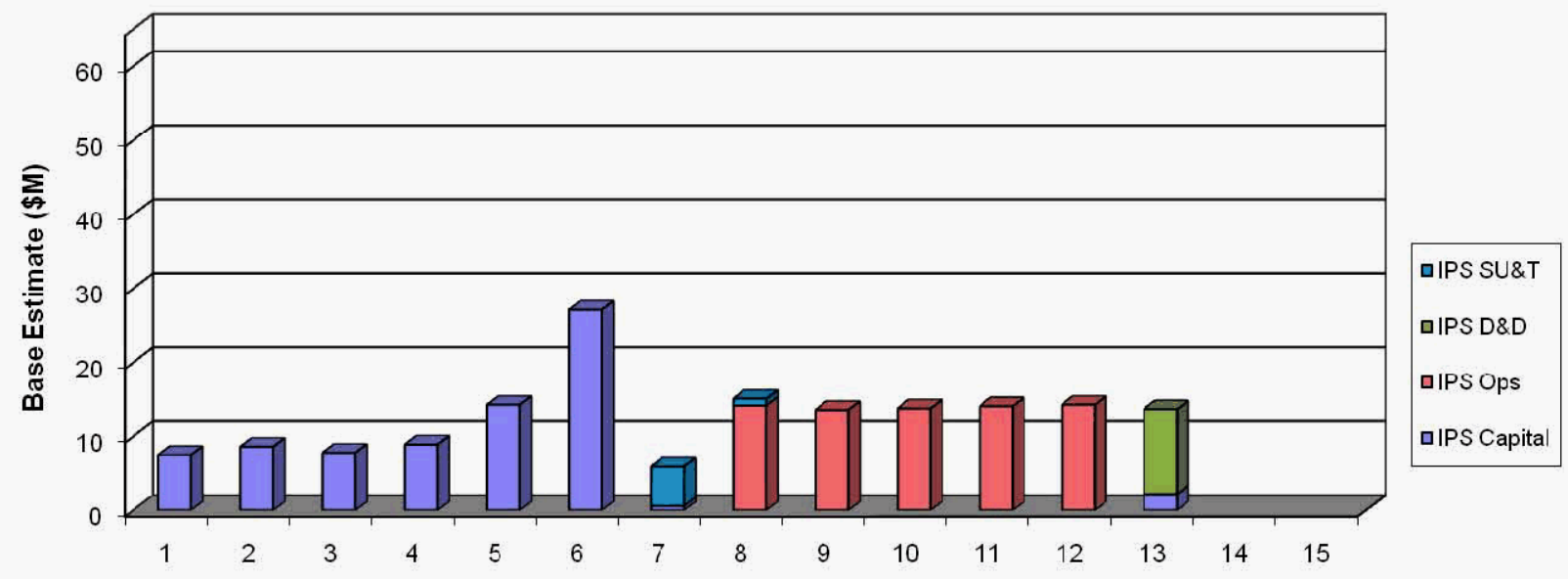

Project Year

Figure 4-3 - Project W-551 Life-Cycle Cost Profile for IX-sRF/CFF 
Project W-551 Base Life Cycle Cost Profile Ion Exchange using spherical Resorcinol-Formaldehyde resin \& Rotary Microfiltration (no expected accuracy range applied)

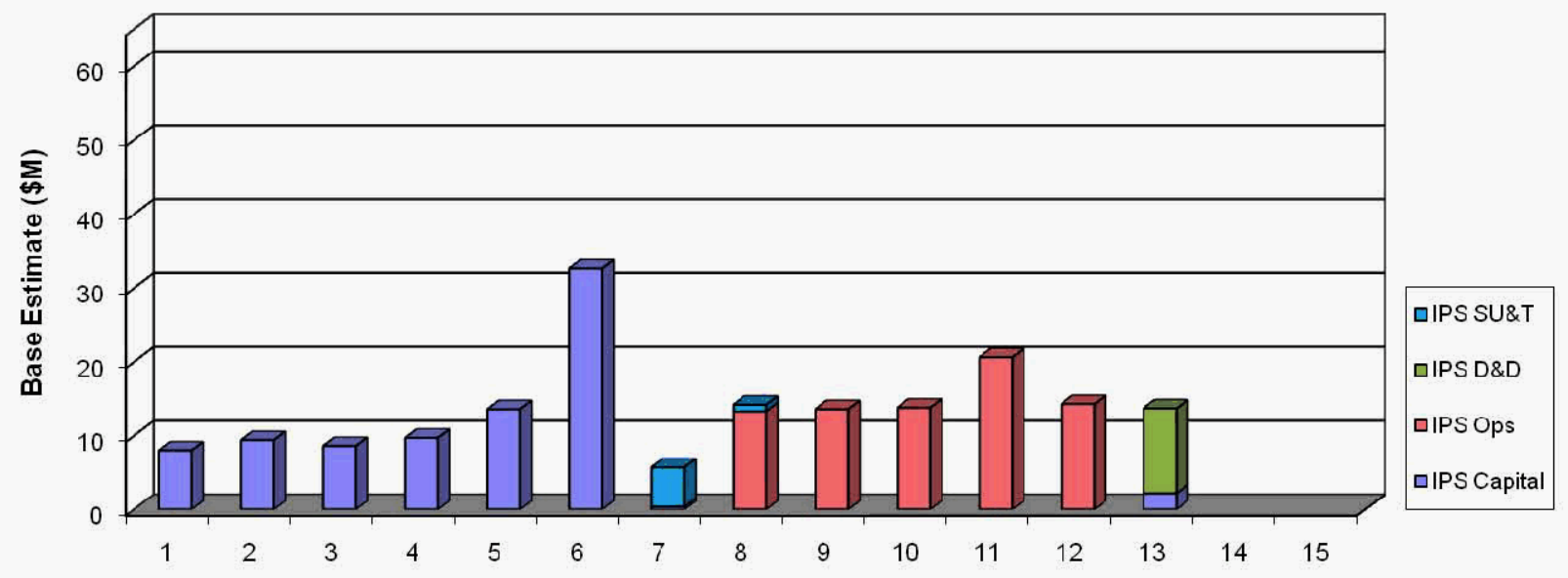

Project Year

Figure 4-4 - Project W-551 Life-Cycle Cost Profile for IX-sRF/RMF 


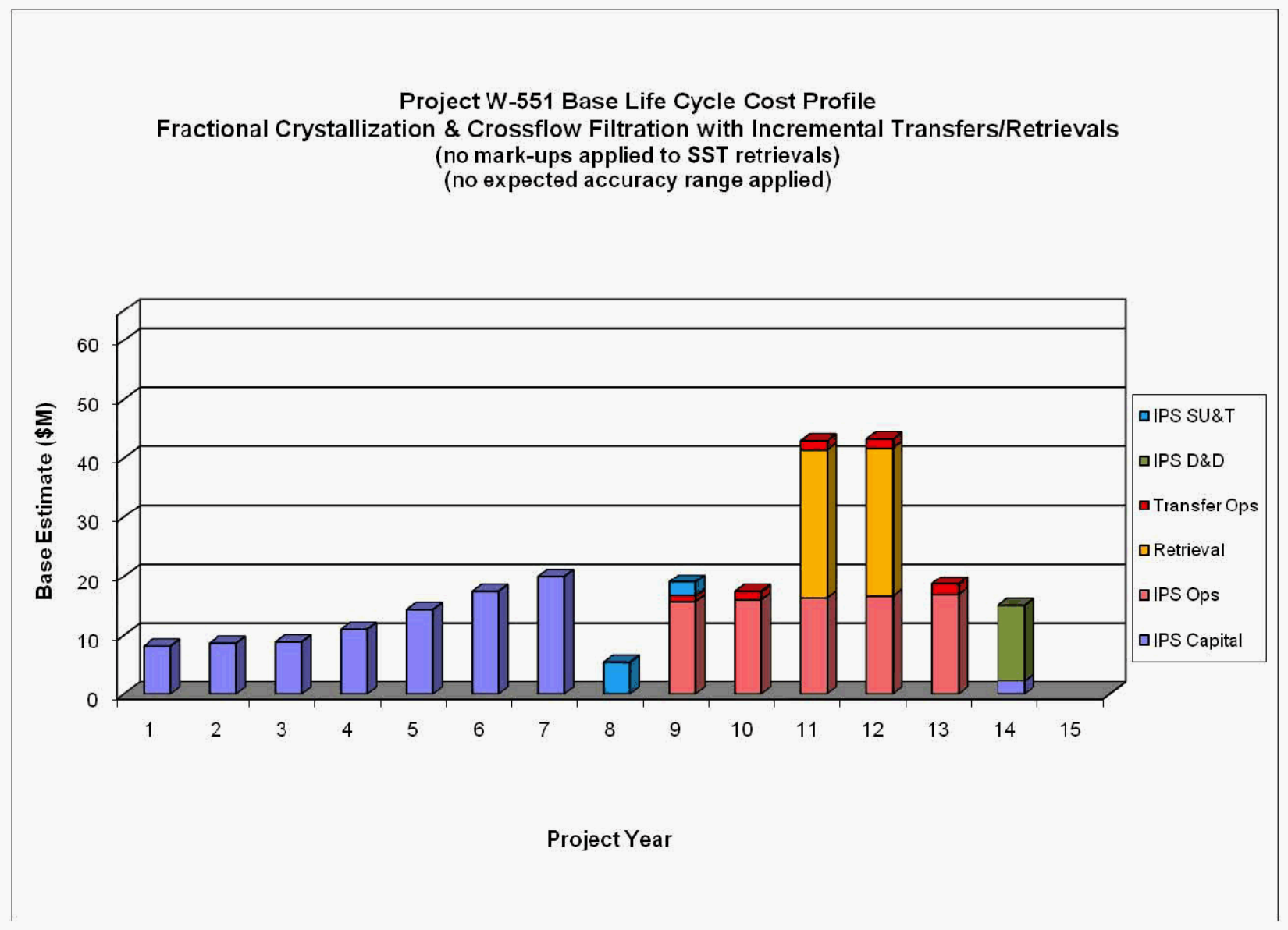

Figure 4-5 - Project W-551 Life-Cycle Cost Profile for FC/CFF 
Project W-551 Base Life Cycle Cost Profile

Fractional Crystallization \& Rotary Microfiltration with Incremental Transfers/Retrievals

(no mark-ups applied to SST retrievals)

(no expected accuracy range applied)

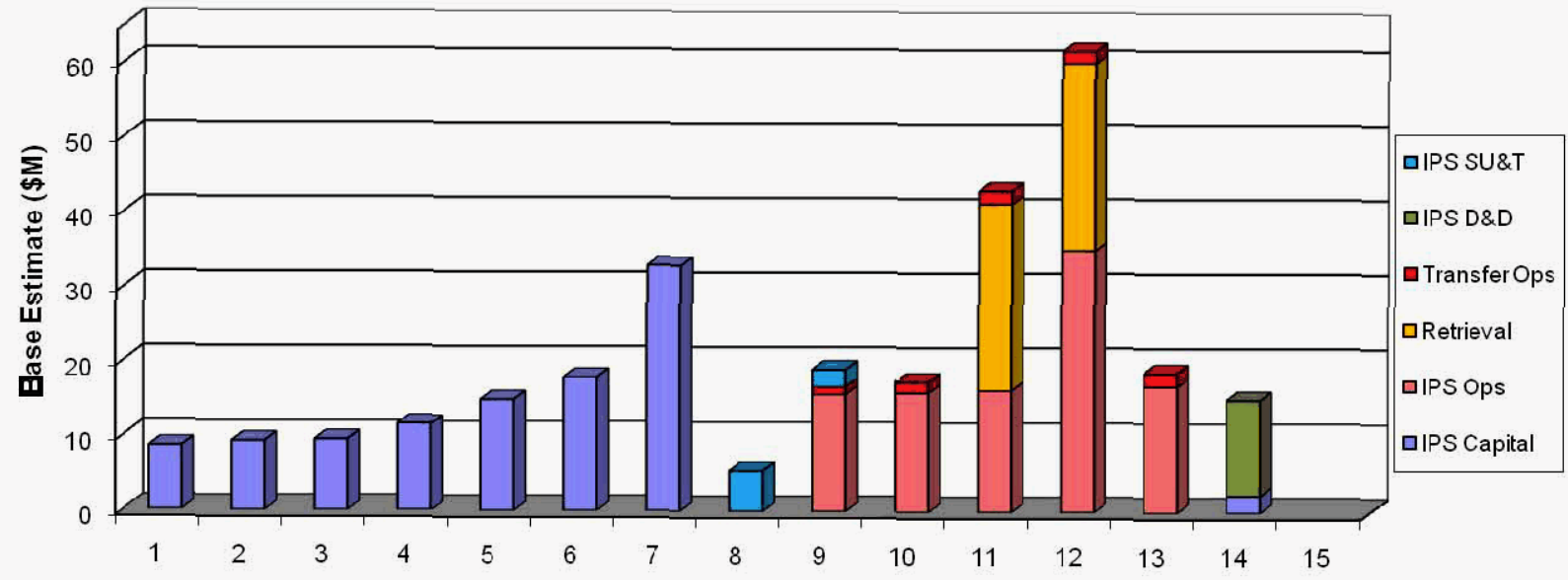

Project Year

Figure 4-6 - Project W-551 Life-Cycle Cost Profile for FC/RMF 


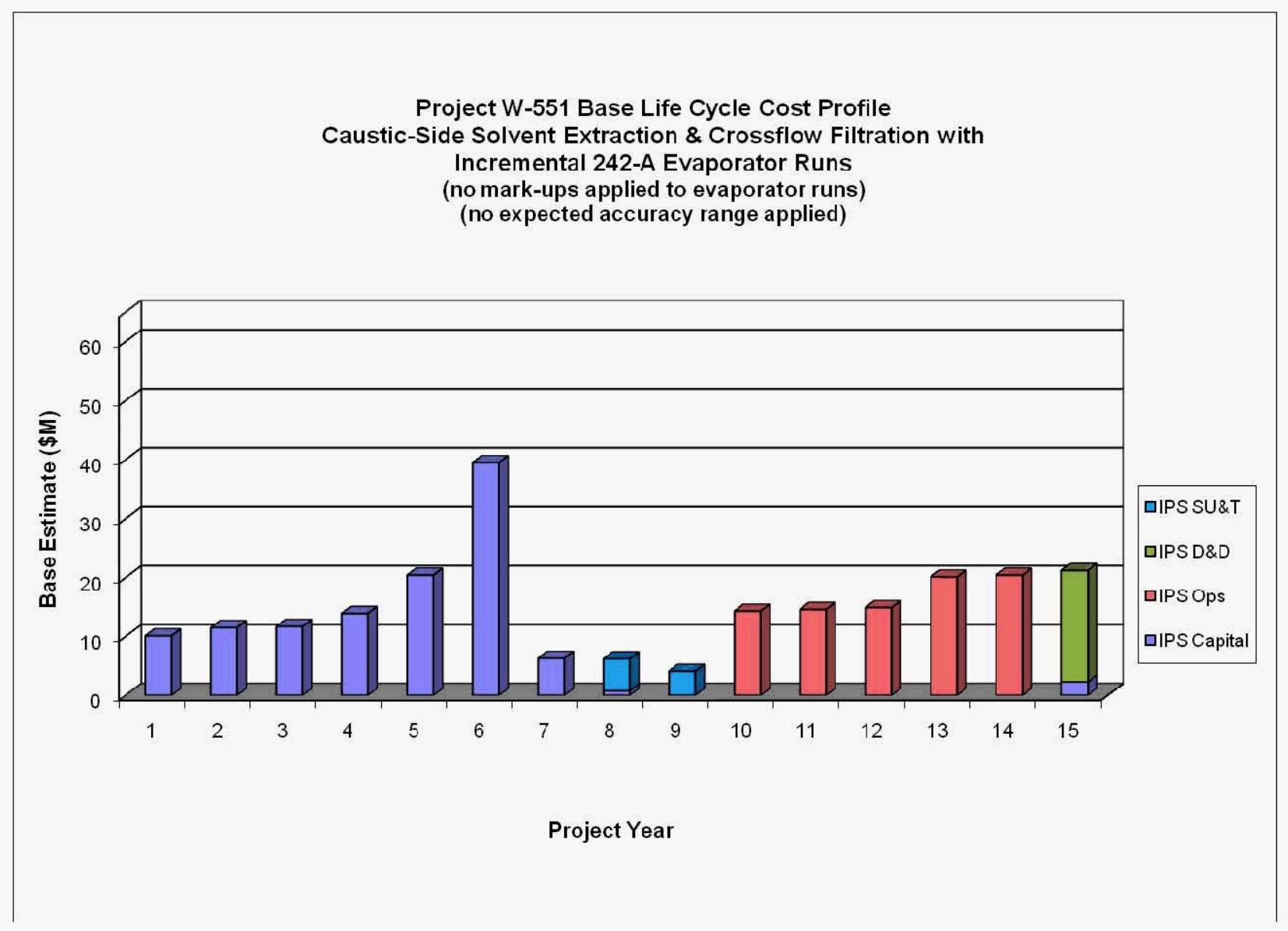

Figure 4-7 - Project W-551 Life-Cycle Cost Profile for CSSX/CFF 


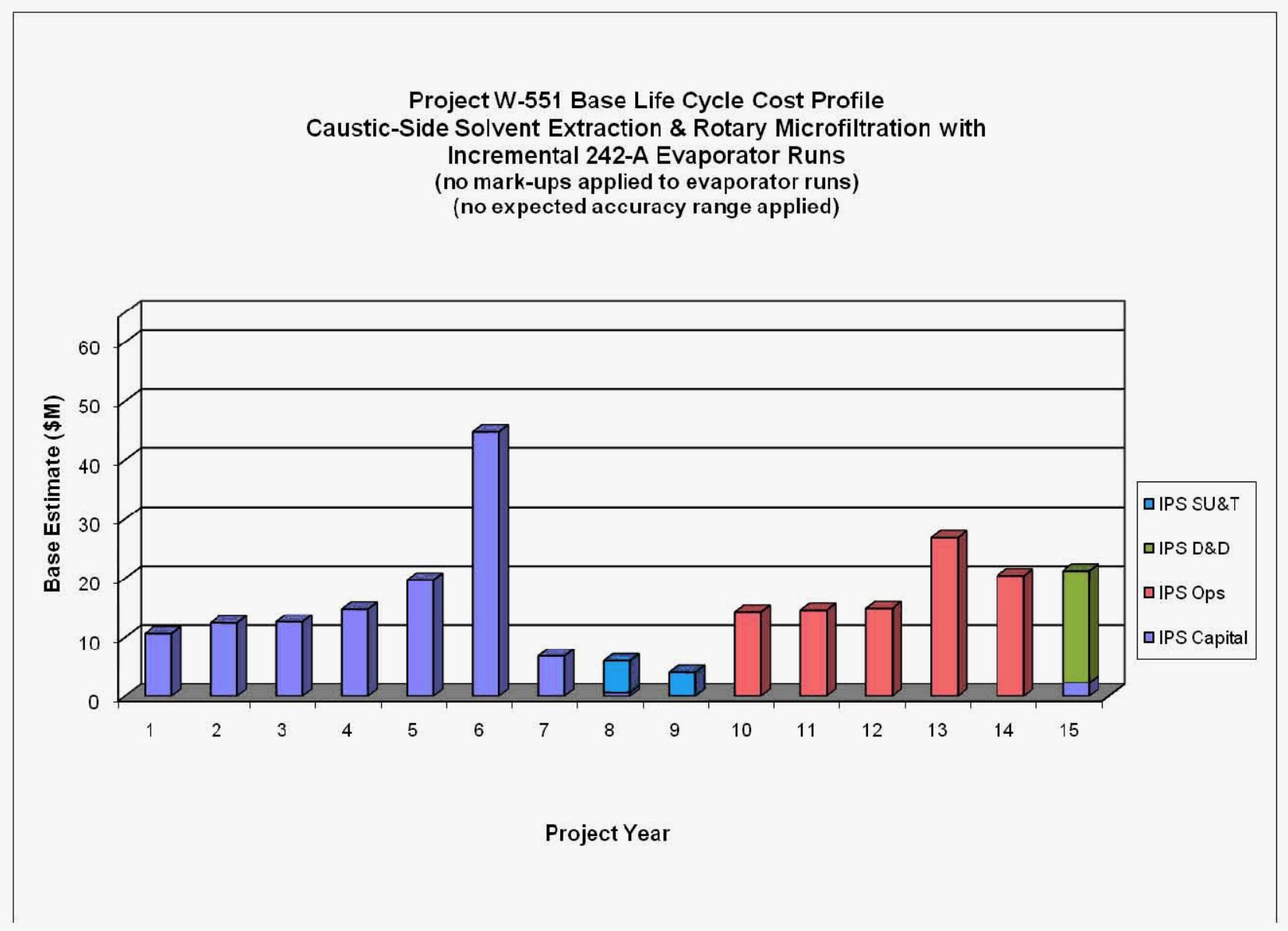

Figure 4-8 - Project W-551 Life-Cycle Cost Profile for CSSX/RMF 


\subsubsection{Schedule Impacts}

In addition to cost, it is important to understand what, if any, impact the candidate cesium separation technologies will have on the IPS implementation schedule. A major question that needs to be answered is whether the IPS Project can be completed in time to allow WTP's LAW facility to operate for a significant period of time prior to startup of WTP's Pretreatment facility. Understanding how the candidate cesium separation technologies impact that issue and whether there are significant differences between them will be key to deciding whether it is worthwhile to go forward with the IPS Project.

The IPS is a major acquisition whose processes must be developed and demonstrated and whose facility must be designed, constructed, permitted, tested and operated in a manner that meets DOE, Federal, State and local requirements and regulations. The major programmatic activities that must be conducted for the IPS project are: engineering and design, regulatory and permitting activities, technology maturity achievement, facility construction, equipment procurement and installation, startup/readiness and cold/ hot operation. Some of these activities are interrelated and will have significant impact on the overall IPS implementation schedule.

In order to better assess the schedule impacts of the candidate technologies, three specific areas of concern were identified, as well as evaluating the overall schedule confidence. The three selected areas were nuclear safety and licensing, permitting, and the D\&D activity. With respect to the three cesium separation technologies, their respective impacts to the IPS implementation schedule are discussed in the sections below.

\subsubsection{Overall Schedule Confidence}

An implementation schedule for IPS has been developed using estimated durations and logic ties for six major activities - design, testing, permitting, safety and licensing, construction, and startup. This schedule also included the four CD milestones (CD-1, CD-2, CD-3, and CD-4) as well as the milestone for the expected issuance of the TC\&WM EIS Record of Decision (expected January 2010). As discussed previously in Section 3.5.2.1, entrained solid separation technologies do not present substantial differences and therefore are not a factor in the overall IPS schedule. Therefore, generic filtration technology activities (representing either RMF or CFF) are included with each cesium separation technology activities.

In order to achieve some level of confidence in an overall IPS schedule and to determine whether any significant schedule differences exist between the three candidate cesium separations technologies, a simplified Monte Carlo simulation approach was selected. To accomplish this simulation, nominal, optimistic ( $<25 \%$ probability of on-time completion), and pessimistic $(>75 \%$ probability of on-time completion) durations for the major activities were estimated for each of the three scheduling cases. Using the 3 duration values (nominal, optimistic, and pessimistic) for each major activity, a Monte Carlo simulation and analysis was performed for each scheduling case.

The results of the Monte Carlo simulation and its analysis show that the overall duration at a $50 \%$ probability of on-time completion of the IPS Project (i.e., ready to start hot operations) is approximately 90 months to fully implement IX-sRF, 100 months for FC, and 110 months for CSSX (see Table 4-6).

The individual evaluations of Overall Schedule Confidence for each of the Cs separation technologies can be found in CSSX-5.2.1, FC-5.2.1, and SRF-5.2.1. 
Table 4-6 - Durations of Major IPS Activities (not additive)

\begin{tabular}{|l|c|c|c|c|}
\hline \multirow{2}{*}{ Activity } & \multicolumn{4}{c|}{ Duration in months } \\
\cline { 2 - 5 } & FC & CSSX & IX-sRF & Remarks \\
\hline \hline Design & 46 & 46 & 46 & \\
\hline Testing & 38 & 38 & 38 & \\
\hline RCRA Permitting & 32 & 32 & 32 & \\
\hline Safety \& Licensing & 46 & 46 & 46 & Duration driven by design. \\
\hline Construction & 33 & 37 & 27 & \\
\hline Startup & 17 & 21 & 14 & \\
\hline Overall Duration & 100 & 110 & 90 & \\
\hline
\end{tabular}

\subsubsection{Licensing}

The primary focus of the safety analysis for the CSSX process will be the numerous centrifugal contactors and the solvent used to effect separation and concentration of the cesium. There has been only minimal experience with these high-speed contactors in an operating environment. The potential flammability of the solvent will also be a priority concern in the safety analysis. Furthermore, the cesium will become concentrated by these contactors, thereby creating higher radiological doses and potential thermal excursions. ANL has performed extensive development and testing of centrifugal contactors, and the ORNL has deployed and successfully used this technology on a small scale. Both of these national laboratories have compiled considerable performance data on the solvent extraction process, which will provide important inputs to the IPS safety analysis activity. Because there is less Hanford-specific knowledge and experience with the CSSX process, there is a lower probability that the IPS safety analysis can be completed on schedule.

The primary focus of the safety analysis for the FC process will be the crystallizer columns, since the cesium will become concentrated on these columns, thereby creating higher radiological doses and potential thermal excursions. However, these characteristics of cesium are well-known and understood, so that analyses of the associated hazards should be straightforward and should not impact the completion of IPS design and start of construction. The FC process operates in a manner much analogous to the 242A Evaporator. These operational similarities will provide important inputs to the IPS safety analysis activity. Because there is considerable experience with the primary source term (Cs) and the FC process operates much like the 242-A Evaporator, the schedule for completing the IPS safety analysis should not be impacted by the FC process.

The primary focus of the safety analysis for the IX-sRF process will be the ion exchange columns, since the cesium will become concentrated on these columns, thereby creating higher radiological doses for the Cs-loaded waste stream that is returned to the tank farms. However, the characteristics of the concentrated cesium are well known and understood and its other properties are quite similar to other tank farm wastes. Additionally the WTP Project is using this same IX resin in its design of the WTP Pretreatment facility, and has compiled considerable performance data on it. These data will provide important inputs to the IPS safety analysis activity. Because there is considerable experience with the primary source term (Cs) and the WTP Project is designing their Pretreatment facility to use the same IX resin, the schedule for completing the IPS safety analysis should not be impacted by the IX-sRF process.

The individual evaluations of Licensing for each of the Cs separation technologies can be found in CSSX5.2.2, FC-5.2.2, and SRF-5.2.2. 


\subsubsection{Permitting}

The key events in assessing whether there will be a schedule impact from any of the cesium separations technologies are the completion of the TC\&WM EIS (expected December 2009) and issuance of its ROD (expected January, 2010). Based on the ROD, an evaluation of the selected cesium separation technology will need to be performed to determine whether it is adequately covered or additional NEPA coverage is required (e.g., EA). Once the NEPA process is completed, the draft RCRA Part B permit can be issued for public review and comment by the regulatory agency. Because the cesium separations technologies generate waste streams that are permitted for treatment (immobilization of low activity waste [ILAW]) or storage (in the DSTs), it is unlikely that any of these technologies will cause significant delays in the RCRA Part B permitting process. In accordance with the Tri-Party Agreement, the overall schedule for the permitting process is $28-33$ months.

The RCRA Part B permit must be approved before IPS construction can start. Assuming that the TC\&WM EIS is issued in December 2009 as expected and a permitting schedule of 28-33 months, permitting of the selected cesium separation technology does not appear to impact IPS start of construction. However, in the event that completion of the TC\&WM EIS is significantly delayed beyond its expected completion date of December 2009, an alternate NEPA option may be required (e.g., an Interim Action EA).

The individual evaluations of Permitting for each of the Cs separation technologies can be found in CSSX-5.2.3, FC-5.2.3, and SRF-5.2.3.

\subsubsection{Decontamination and Decommissioning}

Because the cesium separations equipment will be installed in a newly constructed facility, appropriate considerations for accommodating its eventual D\&D will be included during the design phase of IPS. D\&D of this equipment will most likely occur as part of the IPS D\&D activity following completion of the IPS mission. It is anticipated that design considerations for eventual D\&D of the cesium separations equipment will have negligible impact on the IPS implementation schedule, regardless of the technology selected.

The individual evaluations of Decontamination and Decommissioning for each of the Cs separation technologies can be found in CSSX-5.2.4, FC-5.2.4, and SRF-5.2.4.

\subsubsection{Double-shell Tank Space}

Double-shell tank space is a critical resource in the management of Hanford tank waste that must be managed with care. Liquid radioactive waste handling activities (e.g., retrieval of waste from single-shell tanks) must be carefully evaluated for their potential impact on DST space. From the DST space management point of view, the total net volume of DST space needed or freed up and the annual rate at which DST space is consumed or made available are important. The potential impacts of cesium separation technologies on the DST space are discussed below, both in terms of the rate at which DST space is recovered, as well as the total volume of recovered DST space. Because assessment of the candidate technologies is based and normalized to 5-year IPS mission duration, the rate at which DST space is recovered and the total volume of recovered DST space are directly related.

\subsubsection{How fast is DST space made available}

This section discusses the rate at which DST space is recovered for each of the cesium separations processes under consideration. Using data developed in Attachment G of RPP-RPT-37741 (CH2M HILL 
2008d), the number of batches of waste that are pretreated during the 5-year IPS mission is slightly greater than five for both CSSX and IX-sRF (or approximately one batch per year) and 16 batches for FC (or slightly more than 3 batches per year). Because the WTP solubility limit for aluminum was used in the flow sheet calculations for both CSSX and IX-sRF, the feed batches must first be diluted to $6 \mathrm{M}$ sodium before they can be pretreated by either of these processes. This is considered to be a conservative assumption penalizes these processes in terms of recovered DST space (both rate and total).

Caustic-Side Solvent Extraction (CSSX):

Based on information provided in the CSSX process flow sheets, the as-generated rate at which DST space is recovered $520 \mathrm{~K} \mathrm{gal} /$ year. Additional waste volume reduction is possible, if the current operating limit of $0.8 \mathrm{Ci} /$ liter for the $242-\mathrm{A}$ Evaporator is imposed. Evaporation to the $0.8 \mathrm{Ci} /$ liter limit would create an additional $205 \mathrm{~K} \mathrm{gal} / \mathrm{y}$ ear of DST space, or a total of $725 \mathrm{~K} \mathrm{gal} / \mathrm{year}$. If the higher DSA limit of $1.5 \mathrm{Ci} /$ liter for the evaporator was used, an additional $365 \mathrm{~K} \mathrm{gal} /$ year of DST space, or a total of $885 \mathrm{~K}$ $\mathrm{gal} / \mathrm{year}$ would be generated. The rate of DST space recovery and the total volume of recovered DST space for the CSSX process are shown in the Figure 4-9 below.

The individual evaluation of How Fast is DST Space Made Available using CSSX can be found in CSSX5.3.1.

Figure 4-9 - Net Change in Available Double-Shell Tank Waste Storage Space Solvent Extraction Cesium Separation Technology

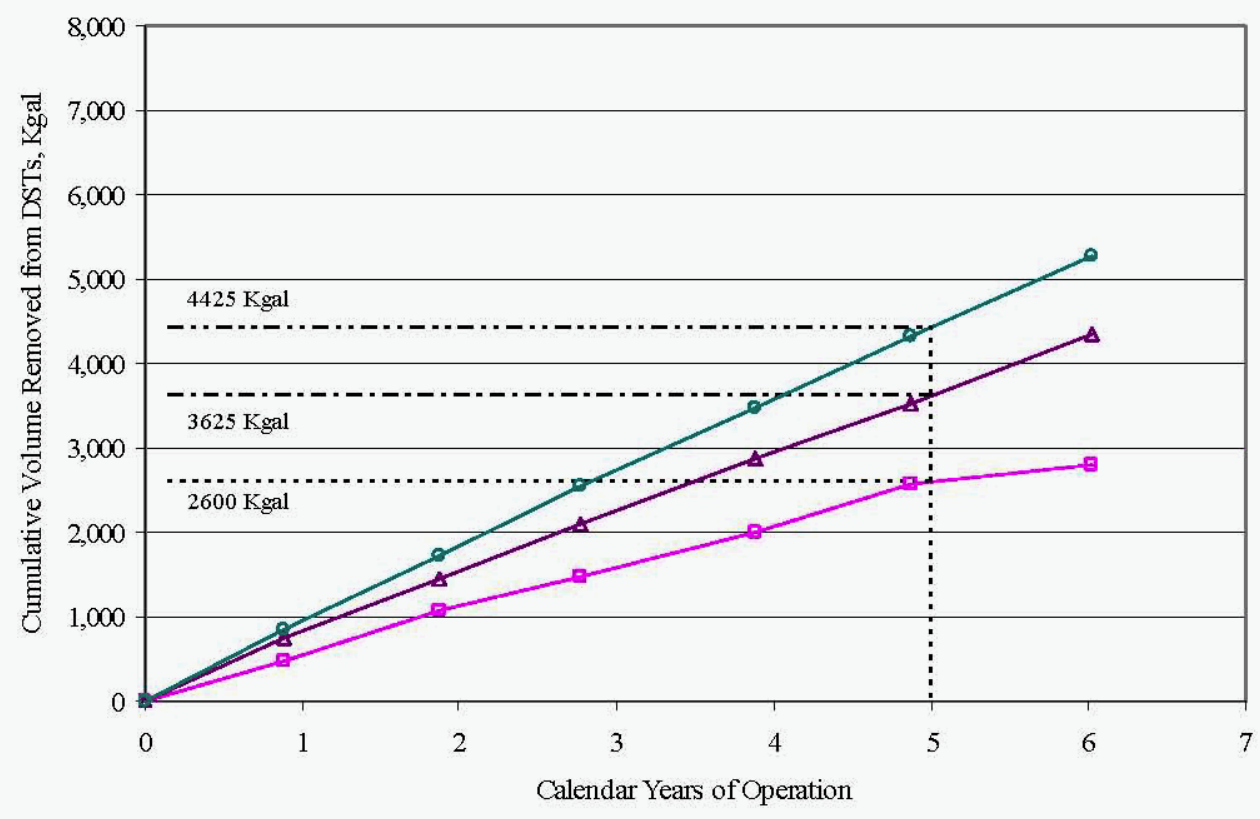

$\rightarrow-$ As Generated Cs Product $\triangle$ Cs Product at $0.8 \mathrm{Ci} / \mathrm{L} \multimap$ Cs Product at $1.5 \mathrm{C} / \mathrm{L}$ 
Fractional Crystallization (FC):

Based on information provided in the FC process flow sheets, the rate at which DST space is recovered during the first 3 years ranges between $\sim 1,300 \mathrm{~K}$ and $\sim 1,500 \mathrm{~K} \mathrm{gal} /$ year, depending on the whether the molarity of the potassium in Cs-loaded stream being returned to the DST system is limited to $0.1 \mathrm{M}$. During the fourth year of pretreatment, the additional amount of DST space recovered ranges between $\sim 600 \mathrm{~K}$ gal and $2,200 \mathrm{~K}$ gal, again depending on to what extent the potassium in the Cs-loaded stream is limited. During the fifth year of pretreatment, the feed batches to FC process are from SSTs, so that DST space is no longer being recovered, but rather consumed. Further volume reduction for any of the cesium-loaded waste streams being returned to the DSTs is not required because the specific gravity for all 8 of these streams meet the current DST specifications. The rate of DST space recovery and the total net volume of recovered DST space for the FC process are shown in the Figure 4-10 below.

The individual evaluation of How Fast is DST Space Made Available using FC can be found in FC-5.3.1.

Figure 4-10 - Net Change in Available Double-Shell Tank Waste Storage Space Fractional Crystallization Cesium Separation Technology

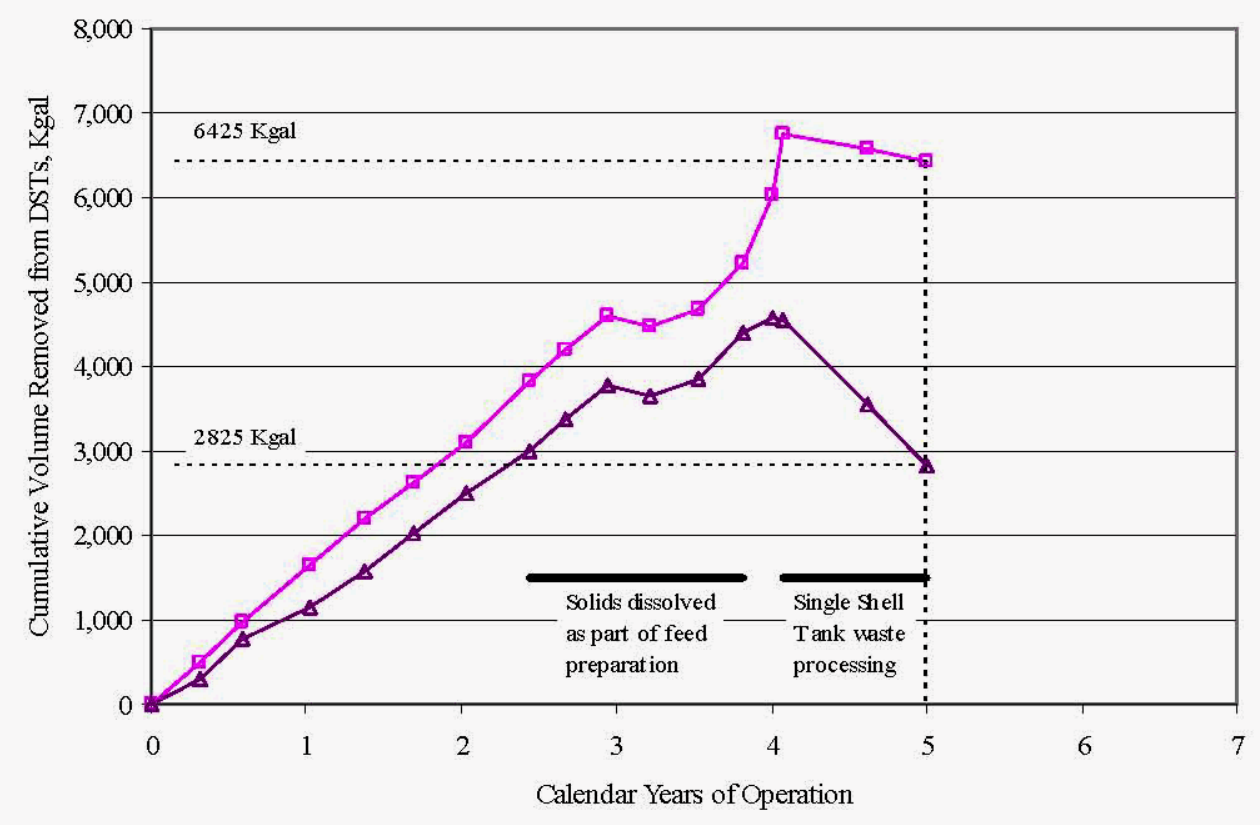

$\rightarrow$ Cs Product Generated at $9 \mathrm{MNa} \triangle \triangle$ Cs Product Limited to $0.1 \mathrm{M}$ Phosphate

Ion Exchange using Spherical Resorcinol-Formaldehyde Resin (IX-sRF):

Based on information provided in the IX-sRF process flow sheets, the as-generated rate at which DST space is recovered $725 \mathrm{~K} \mathrm{gal} /$ year. A small amount of additional DST space $(15 \mathrm{~K}$ gal/year) can be recovered if the current operating limit of $0.8 \mathrm{Ci} /$ liter for the 242-A Evaporator is imposed. If the higher DSA limit of $1.5 \mathrm{Ci} /$ liter for the evaporator was to be used, an additional $205 \mathrm{~K}$ gal/year of DST space, or a total of $930 \mathrm{~K} \mathrm{gal} /$ year would be generated. The rate of DST space recovery and the total volume of recovered DST space for the IX-sRF process are shown in the Figure 4-11 below.

The individual evaluation of How Fast is DST Space Made Available using CSSX can be found in SRF5.3.1. 
Figure 4-11 - Net Change in Available Double-Shell Tank Waste Storage Space Ion Exchange Cesium Separation Technology

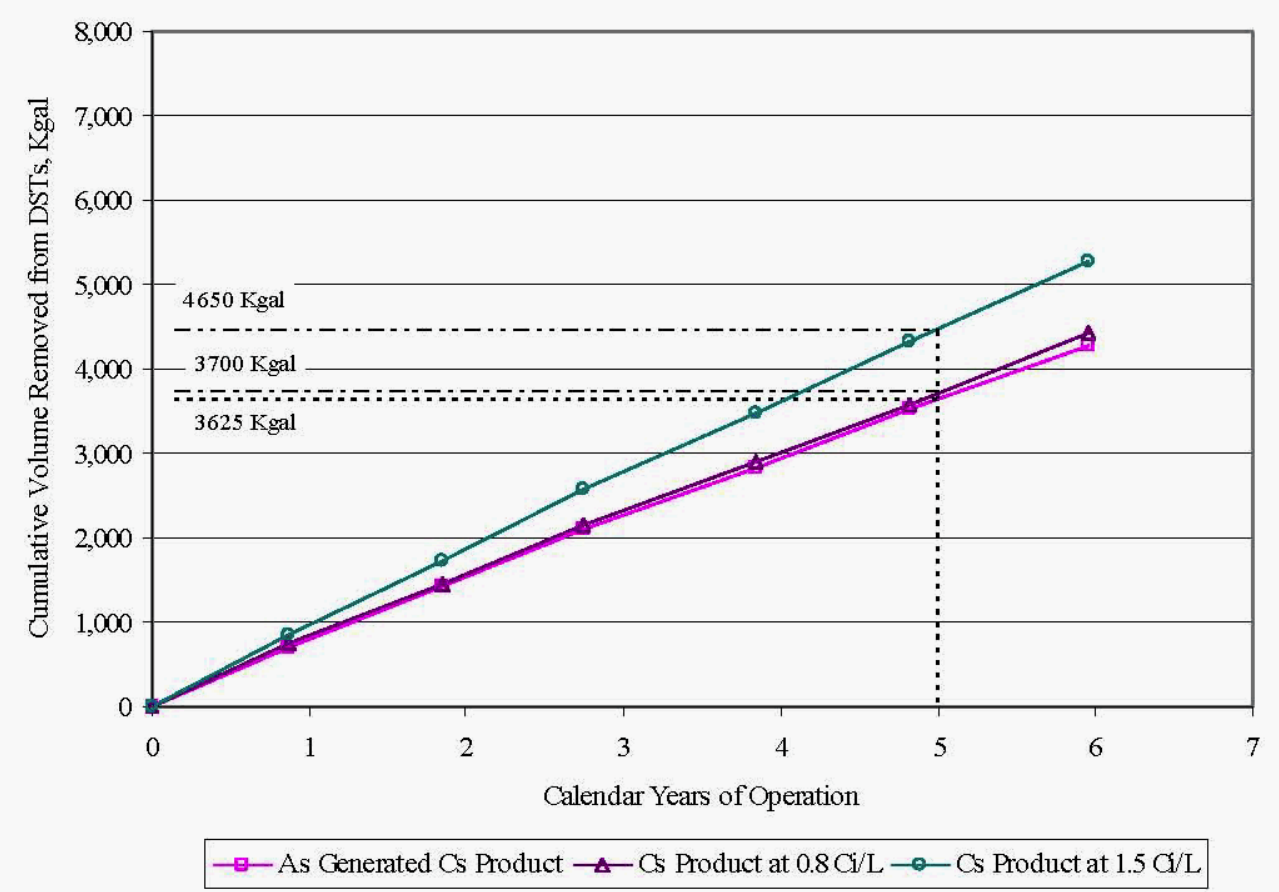

\subsubsection{Total Volume of DST Space Recovered}

As shown in Figures, 4-9, 4-10, 4-11, the total volume of DST space recovered by each of the candidate cesium separations technologies are:

- 3.6M gallons for CSSX (assuming the WTP solubility limit for Al is imposed and the waste volume of the Cs-loaded stream is further reduced at the 242-A evaporator)

- $2.8-6.4 \mathrm{M}$ gallons for FC (depending to what extent the potassium limit of $0.1 \mathrm{M}$ is imposed)

- 3.6M gallons for IX-sRF (assuming the WTP solubility limit for Al is imposed and no further volume reduction at the $242-\mathrm{A}$ evaporator)

The individual evaluations of Total Volume of DST Space Recovered for each of the Cs separation technologies can be found in CSSX-5.3.2, FC-5.3.2, and SRF-5.3.2.

\subsubsection{Impacts to WTP and Supplemental Treatment Plants}

Because the IPS facility provides pretreated wastes to WTP LAW facility and Supplemental Treatment for immobilization, it is important to understand the impacts, positive or negative, of the IPS operation on these facilities. These impacts are driven by quantity and detailed characteristics (chemical conditions, radionuclide inventory, chemical components, etc.) of products delivered by the IPS to these facilities. These impacts can occur in many areas such as production rates, LAW mission duration, number of high and low level packages, lessons learned, technology transfer, ALARA, diversity of technology and positive programmatic impacts and opportunities. These impacts are qualitatively assessed below for the three cesium separation technologies. 


\subsubsection{Production Rate Impact}

The pretreated waste feed prepared for vitrification in the LAW facility may have impact on the production rate of WTP for HLW or LAW facilities. Glass production rate is dependent on the feed composition as well as specific chemical constituents that have positive or negative influence on matrix bindings. Such influence may impact glass quality or change melt rate production (up or down).

Based on preliminary process flowsheet results (CH2M HILL 2008c) all three technologies provides feed to WTP and/or to Supplement Treatment at a rate $0.192 \mathrm{MT} \mathrm{Na} / \mathrm{hr}$ or $1,175 \mathrm{MT} \mathrm{Na} / \mathrm{yr}$ as required by Interim Pretreatment System Mission Scoping Report, RPP-RPT-37644 (CH2M HILL 2008b). This rate is sufficient to provide feed for 2 ILAW melters in WTP and one Supplemental Treatment Line.

In addition to providing a required basic feed rate, feed composition may have positive or negative impacts on production rates of these facilities. Insufficient data are available at this time to assess chemical composition impacts on individual production rates due to each process flowsheet. Detailed assessments of all major chemical constituent of each delivered feed needs to be made to achieve a more complete assessment of the impact of these three cesium separations technologies on WTP production rates.

The individual evaluations of Production Rate Impact for each of the Cs separation technologies can be found in CSSX-5.4.1, FC-5.4.1, and SRF-5.4.1.

\subsubsection{Mission Duration}

The CSSX and IX-sRF ion exchange processes will deliver the 8 tanks $(6,376 \mathrm{MT})$ of processed waste sodium as LAW feed to WTP ( 2 melters) and supplemental Treatment ( 1 Line) over a period of approximately 8 years. Because IPS's IX-sRF process is the same as the baseline pretreatment process for WTP, the required percentage of cold (added non radioactive) $\mathrm{NaOH}$ addition to their respective process feeds will be the same. The CSSX process requires a similar percentage of cold $\mathrm{NaOH}$ addition to its respective process fees, so the impact of using the CSSX process on the overall WTP mission duration is similar to that of using the IX-sRF process.

Conversely even though FC finishes the pretreatment of 8 tanks in less than 3 years, the LAW feed provided by FC only contains $3,142 \mathrm{MT}$ of $\mathrm{Na}$. The remainder of the Na is sent to the tank farm with the high cesium stream product. This high cesium stream is a candidate for HLW processing in the WTP and will undergo further pretreatment for removal of sodium prior to vitrification. The aluminum concentration in the FC-produced high-cesium product stream may be sufficiently high to precipitate aluminum in the DST during the storage. This aluminum may need to be re-dissolved by the addition of $\mathrm{NaOH}$ in WTP Pretreatment to prevent it from being vitrified as part of the HLW stream.

In summary, IX-sRF ion exchange system and CSSX system have a similar mission impact on the WTP. These two processes will reduce the volume of tank wastes that will require processing in the WTP Pretreatment facility when it eventually comes on line. In contrast, the high-cesium product stream produced by the FC process may require significant further pretreatment at WTP before it can be vitrified. However, the mission impact to WTP from the additional processing required by the FC process cannot be fully assessed at this time.

The individual evaluations of Mission Duration for each of the Cs separation technologies can be found in CSSX-5.4.2, FC-5.4.2, and SRF-5.4.2. 


\subsubsection{Number of High and Low-Level Packages}

Assuming a $70 \%$ TOE, the three technologies will provide a total of 5,875 MT Na to WTP and Supplemental Treatment in the 5 years of IPS operation. Assuming that there are no other chemical constituents that would limit the waste oxide loading, the sodium oxide loading for the ILAW glass will be $\sim 20 \%$. Therefore, each container of ILAW produced in WTP will contain approximately 0.75 MT of $\mathrm{Na}$, resulting in $\sim 7,800$ containers of ILAW being produced. However, approximately $30 \%$ of the sodium sent to WTP is added as cold chemical makeup as part of both the IX-sRF and CSSX processes (assuming the WTP solubility limit for aluminum). This number of ILAW canisters would be the same if the proposed feed batches were pretreated in WTP instead of IPS.

The FC pretreatment process has the potential to more than double the number of ILAW containers, if it is unable to adequately remove the sulfate. The presence of sulfates in ILAW feed reduces the waste oxide loading limit, typically to less than $10 \mathrm{wt} \%$.

The individual evaluations of Number of High and Low-Level Packages for each of the Cs separation technologies can be found in CSSX-5.4.3, FC-5.4.3, and SRF-5.4.3.

\subsubsection{Lessons Learned Benefits for WTP Pretreatment}

IX-sRF has been selected as the baseline cesium separation technology for WTP Pretreatment. The operation and maintenance of the IX-sRF resin columns in IPS will provide valuable data and experience for the startup and operation/maintenance of similar equipment in WTP. On the other hand, neither the CSSX nor FC processes will provide significantly meaningful data and experience pertinent to any startup and operation/maintenance in WTP Pretreatment.

The individual evaluations of Lessons Learned Benefits for WTP Pretreatment for each of the Cs separation technologies can be found in CSSX-5.4.4, FC-5.4.4, and SRF-5.4.4.

\subsubsection{Technology Transfer to WTP}

Because IX-sRF has already been selected as the baseline cesium separation technology for WTP Pretreatment, no technology transfer between IPS and WTP Pretreatment will occur. Conversely, the process data and experience gained from the operation and maintenance of either the CSSX or FC processes in IPS could provide valuable information for potentially transferring these technologies to WTP in the event that the baseline technology performance is not satisfactory.

The individual evaluations of Technology Transfer to WTP for each of the Cs separation technologies can be found in CSSX-5.4.5, FC-5.4.5, and SRF-5.4.5.

\subsubsection{ALARA}

The primary source of radioactivity in the supernatant liquids is ${ }^{137} \mathrm{Cs}$. For the 8 waste streams being transferred to WTP or Supplemental Treatment for vitrification, the IX-sRF and the CSSX processes removes ${ }^{137} \mathrm{Cs}$ to a level of $1.68 \mathrm{E}-05 \mathrm{Ci} / \mathrm{g}-\mathrm{mol} \mathrm{Na}$ in the pretreated LAW feed stream going to WTP LAW. This meets the WTP HLW specification of $1.65 \mathrm{E}-05 \mathrm{Ci} / \mathrm{g}-\mathrm{mol} \mathrm{Na}$. The $\mathrm{FC}$ process removes cesium to a level of $1.18 \mathrm{E}-05 \mathrm{Ci} / \mathrm{g}-\mathrm{mol} \mathrm{Na}$ for the worst case waste stream (AP-107). This is approximately $40 \%$ below the WTP specification.

The individual evaluations of ALARA for each of the Cs separation technologies can be found in CSSX5.4.6, FC-5.4.6, and SRF-5.4.6. 


\subsubsection{Diversity of Technology}

IX-sRF has been selected as the baseline cesium separation technology for WTP Pretreatment. Therefore, the use of IX-sRF in IPS does not initially provide an opportunity for diversifying WTP's technology portfolio. However, any early problems with the IX-sRF process or equipment during IPS operations would provide the WTP Project time to evaluate alternative cesium separation technologies prior to WTP start up.

Conversely the use of either the CSSX or FC processes in IPS provides an opportunity for diversifying WTP's technology portfolio. If significant problems were encountered with IX-sRF during its early operation in WTP, the project could then further evaluate deploying either of these cesium separations technologies as an alternative to the IX-sRF process.

The individual evaluations of Diversity of Technology for each of the Cs separation technologies can be found in CSSX-5.4.7, FC-5.4.7, and SRF-5.4.7.

\subsubsection{Positive Programmatic Impacts and Opportunities}

Deployment of IX-sRF technology in IPS will provide opportunities to reduce some of the costs that the WTP Program would otherwise have spent on its development and demonstration. For example, fullscale deployment of IX-sRF in IPS will provide both process and performance data on this technology that WTP would otherwise obtain through its pilot-scale testing program. Furthermore, the IPS experience will be with radioactive wastes, rather than cold simulants, so that technical uncertainties about the IX-sRF ion exchange performance are significantly reduced. The experience gained from the use of IX-sRF ion exchange in IPS will also reduce the uncertainty in WTP's cost estimates for operation and maintenance of this system, and may even allow some acceleration in WTP's startup schedule for its Pretreatment facility.

Because neither CSSX nor FC is the baseline technology for cesium separation in WTP and Supplemental Treatment, there are no programmatic impacts or opportunities associated with either of these technologies.

The individual evaluations of Positive Programmatic Impacts and Opportunities for each of the Cs separation technologies can be found in CSSX-5.4.8, FC-5.4.8, and SRF-5.4.8.

\subsubsection{Impacts to Other Facilities}

The selected cesium separation process may potentially impact other Hanford Site support facilities. In this assessment, impacts to facilities such as the analytical laboratories (222-S Analytical Laboratory and WTP's Analytical Laboratory), ETF, and the 242-A Evaporator operations are evaluated and summarized below.

\subsubsection{Analytical Equipment, Methods and Capacity}

The process descriptions for the three cesium separations processes were reviewed to determine what analytical services would be required to support this process, including characterization of the feed in the candidate feed tanks, the pretreated WTP LAW feed stream (cesium-depleted stream) that is transferred to the WTP LAW facility, and the cesium-loaded stream being transferred back to the DSTs. While the analytical requirements have not been fully defined at this time, it is anticipated that current analytical capabilities (analytical methods and capacity) will be adequate to meet the analytical needs of any of the cesium separations processes. 
It is expected that the WTP analytical laboratory will be used to analyze the LAW feed product to ensure that each batch meets the feed specifications. While the requirements (e.g., number and frequency) for both the waste feed to IPS from the candidate tanks and the cesium-loaded stream have not yet been fully defined, the 222-S Analytical Laboratory should be capable of supporting any analyses identified for these waste streams. It was also assumed that any process control sampling and analysis would be performed in-line or at the IPS facility.

The total turnaround times typically required for sampling, sampling transport, and analyses of the product streams appear to exceed the IPS lag storage capacity. The amount of lag storage may need to be adjusted, if the current typical turnaround times for sampling and analysis cannot be shortened.

The individual evaluations of Analytical Equipment, Methods and Capacity for each of the Cs separation technologies can be found in CSSX-5.5.1, FC-5.5.1, and SRF-5.5.1.

\subsubsection{Compliance to ETF WAC}

A review of the process flow diagrams for both the CSSX and IX-sRF ion exchange processes showed that there are no process condensates from either of these processes requiring disposal at the ETF. Because they do not generate any liquid effluents, neither CSSX nor IX-sRF has any direct impact on the ETF operation. If the cesium-loaded stream from either the CSSX or IX-sRF process requires volume reduction at the 242-A evaporator, these campaigns will generate liquid effluents requiring disposal at ETF. It is anticipated that those effluents would necessarily meet ETF Waste Acceptance Criteria (WAC).

A similar review of the process flow diagram for the FC process showed that there is a significant volume of process condensates from this process that will be sent directly to the ETF. The volumes, compositions, and concentrations of these condensates are estimated in the mass balance calculations. Key constituents of these condensates were compared to their respective waste acceptance values for ETF in the attached table and were found to be within the WAC limits for ETF.

The individual evaluations of Compliance to ETF WAC for each of the Cs separation technologies can be found in CSSX-5.5.2, FC-5.5.2, and SRF-5.5.2.

\subsubsection{ALARA}

Because the selected cesium separations process will be incorporated into a new facility for IPS, the ALARA principle will be proactively addressed during the IPS facility design and the cesium separation process design to ensure that WAC for supporting facilities are met, less hazardous/less toxic materials that would potentially impact supporting facilities are used wherever possible, and sampling/analysis requirements at the analytical laboratories are minimized.

The individual evaluations of ALARA for each of the Cs separation technologies can be found in CSSX5.5.3, FC-5.5.3, and SRF-5.5.3.

\subsubsection{Number of 242-A Evaporator Campaigns}

All of the cesium separations processes generate a cesium-loaded stream that will be stored in the DST system until the WTP Immobilized High-Level Waste facility is ready to vitrify it. These cesium-loaded streams require DST storage space and must meet DST storage specifications.

Based on information provided in the CSSX process flow sheets, the cesium concentrations of the cesium-loaded streams from the CSSX process are sufficiently below the current 242-A Evaporator 
operating limit of $0.8 \mathrm{Ci} /$ liter that waste volume reduction would be beneficial for each of the 5 batches of CSSX-pretreated waste. As shown in Figure 4-9, additional volume reduction of these streams could be realized through evaporation if the DSA limit for the $242-\mathrm{A}$ Evaporator of $1.5 \mathrm{Ci} / \mathrm{Liter}$ was imposed.

Based on information provided in the FC process flow sheets, the specific gravity of the cesium-loaded stream resulting from the FC process is estimated to be $1.41 \mathrm{~g} / \mathrm{cc}$. Since density value meets the current DST specification for specific gravity, no additional volume reduction is required. Therefore, no evaporator campaigns will be required to support the FC technology option.

Based on information provided in the IX-sRF ion exchange process flow sheets, the cesium concentrations of the cesium-enriched streams resulting from this process either exceeded the 242-A evaporator operating limit of $0.8 \mathrm{Ci} /$ liter or were near the limit. Therefore, there would be little benefit gained, i.e., only a small volume of additional DST space would be recovered, and no evaporator campaigns are anticipated. As shown in Figure 4-11, additional volume reduction of these streams could be realized through evaporation if the DSA limit for the 242-A Evaporator of $1.5 \mathrm{Ci} / \mathrm{Liter}$ was imposed.

The individual evaluations of Number of 242-A Evaporator Campaigns for each of the Cs separation technologies can be found in CSSX-5.5.4, FC-5.5.4, and SRF-5.5.4.

\subsubsection{Resources and Materials}

An assessment of processes was executed to look at resource and material specialty required by each cesium separation processes for an overall implementation. Engineering resources are assumed to be at premium for an execution of such a large project at the Hanford Site when other large projects are also being implemented. But such resource competition will exist regardless of the technology selected. A table of all major resources was developed for each technology and appropriate assessments are made against various key factors such as quantity, frequency, suppliers, etc. The table is provided with the assessment forms.

CSSX process implementation requires centrifugal contractors. At this time, only one experienced and knowledgeable supplier (Costner Industries Nevada Corp.) exists. Other manufacturers are not experienced in IPS type applications and may require a learning curve to be competitive. In addition, the CSSX process also uses some proprietary and specialized organic solvents. These include Isopar L, BobCalixC6, Cs-7SB, etc. Premium pricing may have to be paid and proper availability must be guaranteed for the long-term viability and operation of this process.

Supply of the SRF resin is the most critical component of this process. WTP project has done significant planning work in acquiring this material. Two facilities have been identified and some product tests have been completed. Tests showed that product met all test requirements. A long-term resin supply strategy, similar to WTP, must be required to be developed for implementation of this process.

The FC system requires no special material or equipment. Engineered equipment can be designed and built by multiple vendors with some having a more experience than others. No specific difficulty is anticipated for this system from material procurement point of view. No specialty chemicals are used in the process.

For all three technologies, qualified equipment fabricator(s) may be hard to find, and premium prices may have to be paid.

The individual evaluations of Resources and Materials for each of the Cs separation technologies can be found in CSSX-5.6.1, CSSX-5.6.2, FC-5.6.1, FC-5.6.2, SRF-5.6.1, and SRF-5.6.2. 


\subsection{REFERENCES}

24590-WTP-RPT-PT-02-005, 2003, Flowsheet Bases, Assumptions, and Requirements, P. Rutland and S. Sanders, Bechtel National Inc., Richland Washington.

24590-WTP-RPT-RT-07-005, 2008, Basis of Recommendation for Use of Spherical Resorcinol Formaldehyde Resin as the Primary Cesium Ion Exchange Resin in the WTP, Rev 0, Bechtel National, Inc., Richland, Washington.

ANL-00/31, 2001, Temperature Management of Centrifugal Contactors for Caustic-Side Solvent Extraction of Cesium from Tank Waste, Argonne National Laboratory, Chicago, Illinois.

BNF-003-98-0221, 2000, Final Report: Pilot-scale Cross-flow Ultrafiltration Test Using a Hanford Site Tank 241-AN-105 Waste Simulant-Envelope A + Entrained Solids, Westinghouse Savannah River Company, Aiken, South Carolina.

BNF-003-98-0226, 2000, Final Report: Pilot-scale Cross-flow Ultrafiltration Test Using a Hanford Site Tank 24I-AN-107 Waste Simulant-Envelope C + Entrained Solids + Strontium-Transuranic Precipitation, Westinghouse Savannah River Company, Aiken, South Carolina.

CH2M HILL 2006a, Hamilton, D. W., HanfordMedium/Low Curie Waste Pretreatment ProjectPretreatment Process Plan, Rev. 0, RPP-PLAN-27238, January 18, 2006, CH2MHILL Hanford Group, Inc., Richland, Washington.

CH2M HILL 2006b, Hamilton, D. W., Hanford Medium/Low Curie Waste Pretreatment Project-Phase I Laboratory Report, Rev. 0, RPP-RPT-27239, January 30, 2006, CH2M HILL Hanford Group, Inc., Richland, Washington.

CH2M HILL 2006c, Johnson, M. E., Supporting Information for the Evaluation of Starting the Waste Treatment and Immobilization Plant (WTP) Low Activity Waste (LAW) Startup First Scenarios, Rev. 0, RPP-RPT-30160, September 11, 2006, CH2M HILL Hanford Group, Inc., Richland, Washington.

CH2M HILL 2007, Thien, M. G., Evaluation of Starting the Waste Treatment and Immobilization Plant (WTP) Low Activity Waste (LAW) Facility First, RPP-29981, Rev. 1, March 2007, CH2M HILL Hanford Group, Inc., Richland, Washington.

CH2M HILL 2008a, May, T. H. et al, Decision Plan: Selection of Early LAW Interim Pretreatment System Processes for Removal of Entrained Solids and Cesium, Rev. 0, RPP-PLAN-37558, May 5, 2008, CH2M HILL Hanford Group Inc., Richland, Washington.

CH2M HILL 2008b, Thien, M. G., Interim Pretreatment System Mission Scoping Report, Rev 0, RPPRPT-37644, May 21, 2008, CH2MHILL Hanford Group, Inc., Richland, Washington.

CH2M HILL 2008c, May, T. H. et al, Project W-551 Interim Pretreatment System Pre-Conceptual Candidates Technology Descriptions, Rev. 0, RPP-RPT-37551, June 12, 2008, CH2M HILL Hanford Group Inc., Richland, Washington.

CH2M HILL 2008d, Tedeschi, A. R. et al, Project W-551 Determination Data for Early LAW Interim Pretreatment System Selection, Rev. 0, RPP-RPT-37741, July 2008, CH2M HILL Hanford Group Inc., Richland, Washington.

CH2M HILL 2008e, Conrad, E. A., Project W-551 Interim Pretreatment System Technology Selection Summary Decision Report and Recommendation, Rev. 0, RPP-RPT-38057, July 2008, CH2M HILL Hanford Group Inc., Richland, Washington. 
Clean Air Act of 1997, 42 USC 7401, et seq.

Clean Water Act of 1997, 33 USC 1251, et seq.

Delmau, L. H., Haverlock, T. J., Bazelaire, E., Bonnesen, P. V., Ditto, M. E., and B. A. Moyer, submitted for publication April 2008, Alternatives to Nitric Acid Stripping in the Caustic-Side Solvent Extraction (CSSX) Process for Cesium Removal from Alkaline High-Level Waste, Solvent Extraction and Ion Exchange.

DOE/ORP-2007-01, 2007, Technology Readiness Assessment for the Supplemental Treatment Program, U.S. Department of Energy, Office of River Protection, Richland, Washington.

DOE 2008, Technology Readiness Assessment (TRA)/ Technology Maturation Plan (TMP) Process Guide, March 2008, US DOE Office of Environmental Management, Washington, D.C.

DOE Order 420.1B, 2007, Facility Safety, U.S. Department of Energy, Washington D.C.

LWO-SPT-2007-000245, 2007, Scale-up of Caustic-Side Solvent Extraction Process for Removal of Cesium at Savannah River Site, Washington Savannah River Company, Aiken, South Carolina.

National Emission Standards for Hazardous Air Pollutants (NESHAP), 40 CFR Part 61, et seq.

National Environmental Policy Act of 1969, 42 USC 4321, et seq.

ORNL/TM-2002/190, 2002, Caustic-Side Solvent Extraction: Chemical and Physical Properties of the Optimized Solvent, Oak Ridge National Laboratory, Oak Ridge, Tennessee.

ORNL/TM-2004/59, 2004, Conceptual Design of a Simplified Skid-Mounted Caustic-Side Solvent Extraction Process for Removal of Cesium from Savannah River Site High-Level Waste, Oak Ridge National Laboratory, Oak Ridge, Tennessee.

ORP 2008, C.B. Reid to D.B. Cartmell, Initiation Mission Analysis and Preliminary Conceptual Design for Interim Pretreatment System (IPS), Letter 08-AMD-050, March 10, 2008.

RCW 43.21C, "Washington State Environmental Policy Act," Revised Code of Washington, as amended, Olympia, Washington.

Resource Conservation and Recovery Act of 1976, 42 USC 6901, et seq.

WAC 173-303-806, "Dangerous Waste Regulations: Final facility permits," Washington Administrative Code, as amended.

WAC 173-303-840, "Dangerous Waste Regulations: Procedures for decision making," Washington Administrative Code, as amended.

WSRC-MS-2006-00115, 2006, Cross-Flow Ultrafiltration Scaling Considerations, Westinghouse Savannah River Company, Aiken, South Carolina.

WSRC-STI-2006-00073, 2006, Testing and Evaluation of the Modified Design of the 25 Disk Rotary Microfilter, Westinghouse Savannah River Company, Aiken, South Carolina.

WSRC-STI-2007-00580, 2007, Full-Scale Testing of a Caustic Side Solvent Extraction System to Remove Cesium from Savannah River Site Radioactive Waste, Rev. 0, Savannah River National Laboratory, Aiken, South Carolina.

WSRC-STI-2008-00050, 2008, Development of a Rotary Microfilter for Radioactive Waste Applications, Westinghouse Savannah River Company, Aiken, South Carolina. 
WSRC-TR-2002-00243, 2002, High Level Waste Demonstration of Caustic-Side Solvent Extraction Process with Optimized Solvent in the 2-cm Centrifugal Contactor Apparatus using Tank $37 H / 44 F$ Supernate, M. A. Norato et al, Westinghouse Savannah River Company, Aiken, South Carolina.

WSRC-TR-2002-00307, 2002, Demonstration of Caustic Side Solvent Extraction with Optimized Solvent in the 2-cm Centrifugal Contactor Apparatus using Dissolved Salt Cake from Tank 37H, M. A. Norato et al, Westinghouse Savannah River Company, Aiken, South Carolina.

WSRC-TR-2002-00530, 2003, Filtration of a Hanford AW-101 Waste Sample, M. R. Poirier et al, Westinghouse Savannah River Company, Aiken, South Carolina.

WSRC-TR-2003-00295, 2003, Filtration of a Hanford AN-104 Waste Sample, M. R. Poirier et al, Westinghouse Savannah River Company, Aiken, South Carolina.

WSRC-TR-2005-00182, 2005, Examination of Organic Carryover from 2-cm Contactors to Support the Modular CSSX Unit, Rev. 0, Westinghouse Savannah River Company, Aiken, South Carolina.

WSRC-TR-2005-00258, 2005, Waste and Solvent Composition Limits for Modular Caustic-Side Solvent Extraction Unit (MCU), Rev. 0, Westinghouse Savannah River Company, Aiken, South Carolina. 


\section{ATTACHMENT A}

DECISION CRITERIA, MEASURES, AND DEFINITIONS FOR INTERIM PRETREATMENT SYSTEM TECHNOLOGIES 


\begin{tabular}{|c|c|c|c|c|c|c|c|}
\hline \multicolumn{8}{|c|}{ Attachment A - Decision Criteria, Measures, and Definitions For IPS Technologies } \\
\hline No. & Criteria & \begin{tabular}{|l|} 
Weight \\
Factor \\
\end{tabular} & No. & Measures & \begin{tabular}{|l|} 
Ranking \\
Factor
\end{tabular} & No. & Definitions \\
\hline 1 & Safety & 25 & 1.1 & \begin{tabular}{|l} 
Process Safety \\
\end{tabular} & 25 & $\begin{array}{l}1.1 .1 \\
1.1 .2 \\
1.1 .3 \\
1.1 .4 \\
1.1 .5 \\
1.1 .6 \\
1.1 .7 \\
1.1 .8 \\
1.2 .1\end{array}$ & $\begin{array}{l}\text { Quantity of material at risk (MAR) - } \\
\text { radiological and chemical - less is better } \\
\text { Concentration of radiological and chemical } \\
\text { MAR - less is better } \\
\text { Dispersability of the MAR - less dispersible } \\
\text { form is better (e.g., solids over liquids over } \\
\text { powders over gases) } \\
\text { Dispersive energy, e.g., heat, off gassing, } \\
\text { pressure, etc. inherent in the process - less } \\
\text { dispersive energy is better } \\
\text { Process Stability - including ease of process } \\
\text { control/shutdown -- easier/faster process } \\
\text { shutdown is better } \\
\text { Process that does not create a new or } \\
\text { exacerbate an existing Tank Farm hazard is } \\
\text { preferred to one that does } \\
\text { Less fire hazard (e.g. less quantity of } \\
\text { combustibles, including flammable gas, less } \\
\text { flammable combustibles, etc.) } \\
\text { Reactive Chemicals - Process with less } \\
\text { reactive chemicals (reactivity) is better } \\
\text { A Process that is inherently sub critical is } \\
\text { preferred over a process that relies on } \\
\text { criticality controls } \\
\text { Less hazards/less severe hazardous is better } \\
\text { (e.g., less hazardous chemicals, less noise, less } \\
\text { hot surfaces, less rotating equipment, less } \\
\text { confined spaces, etc.) }\end{array}$ \\
\hline 2 & $\begin{array}{l}\text { Regulatory/ } \\
\text { Stakeholder } \\
\text { Acceptance }\end{array}$ & 20 & 2.1 & $\begin{array}{l}\text { Achieve Tribal } \\
\text { Nations/stakeholders' } \\
\text { acceptance }\end{array}$ & 40 & 2.1 .1 & Early waste treatment enabled \\
\hline
\end{tabular}




\begin{tabular}{|c|c|c|c|c|c|c|c|}
\hline \multicolumn{8}{|c|}{ Attachment A - Decision Criteria, Measures, and Definitions For IPS Technologies } \\
\hline No. & Criteria & $\begin{array}{l}\text { Weight } \\
\text { Factor }\end{array}$ & No. & Measures & \begin{tabular}{|l} 
Ranking \\
Factor
\end{tabular} & No. & Definitions \\
\hline & & & 2.2 & $\begin{array}{l}\text { Achieve regulators' } \\
\text { acceptance }\end{array}$ & 60 & $\begin{array}{l}2.1 .2 \\
2.2 .1 \\
2.2 .2 \\
2.2 .3 \\
2.2 .4\end{array}$ & $\begin{array}{l}\text { Land usage (more contaminated ground) } \\
\text { Compliance with applicable regulations } \\
\text { (RCRA, NEPA/SEPA, NESHAPS, NPDES, } \\
\text { CAA, DOE Orders) } \\
\text { Impact to Disposal System Performance } \\
\text { Secondary Waste Form and Quantity } \\
\text { Potential impacts to other permitted facilities }\end{array}$ \\
\hline 3 & $\begin{array}{l}\text { Technical } \\
\text { Maturity/Flexibility }\end{array}$ & 20 & 3.2 & $\begin{array}{l}\text { Technology Readiness } \\
\text { Level } \\
\text { Process Flexibility and } \\
\text { robustness }\end{array}$ & 40 & $\begin{array}{l}3.1 .1 \\
3.1 .2 \\
3.1 .3 \\
3.2 .1 \\
3.2 .2 \\
3.2 .3 \\
3.2 .4 \\
3.2 .5 \\
3.2 .6\end{array}$ & $\begin{array}{l}\text { TRL Number } \\
\text { Effort to mature technology (cost and } \\
\text { schedule) } \\
\text { Probability of Success } \\
\text { Ability to process a variety of feeds } \\
\text { Ability to adjust process rate } \\
\text { Flexibility to modify product } \\
\text { Ability to expand } \\
\text { Recover from out of spec product } \\
\text { Technology applicability to other DOE } \\
\text { complex projects }\end{array}$ \\
\hline 4 & $\begin{array}{l}\text { Operability and } \\
\text { Maintainability }\end{array}$ & 15 & 4.1 & $\begin{array}{l}\text { Ease of Process control } \\
\text { and operation }\end{array}$ & 18 & $\begin{array}{l}4.1 .1 \\
4.1 .2 \\
4.1 .3 \\
4.1 .4 \\
4.1 .5 \\
4.1 .6 \\
4.1 .7 \\
4.1 .8 \\
4.1 .9\end{array}$ & $\begin{array}{l}\text { Minimize number and frequency of } \\
\text { surveillances } \\
\text { Minimize number of people to operate } \\
\text { Ease of startup and shutdown } \\
\text { Minimize system complexity } \\
\text { Minimize number of chemicals needed } \\
\text { Minimize number of process and regulatory } \\
\text { samples } \\
\text { Batch verses continuous } \\
\text { Ease of entry and exit from standby } \\
\text { Wide operating margin }\end{array}$ \\
\hline
\end{tabular}




\begin{tabular}{|c|c|c|c|c|c|c|c|}
\hline \multicolumn{8}{|c|}{ Attachment A - Decision Criteria, Measures, and Definitions For IPS Technologies } \\
\hline No. & Criteria & $\begin{array}{l}\text { Weight } \\
\text { Factor }\end{array}$ & No. & Measures & $\begin{array}{l}\text { Ranking } \\
\text { Factor }\end{array}$ & No. & Definitions \\
\hline & & & $\begin{array}{l}4.2 \\
4.3 \\
4.4\end{array}$ & $\begin{array}{l}\text { ALARA } \\
\text { Reliability } \\
\text { Ease and frequency of } \\
\text { maintenance }\end{array}$ & $\begin{array}{l}26 \\
16 . \\
16\end{array}$ & $\begin{array}{l}4.1 .10 \\
4.2 .1 \\
4.3 .1 \\
4.3 .2 \\
4.4 .1 \\
4.4 .2 \\
4.4 .3 \\
4.4 .4 \\
4.4 .5 \\
4.5 .1 \\
4.5 .2 \\
4.5 .3 \\
4.6 .1 \\
4.6 .2\end{array}$ & $\begin{array}{l}\text { Complexity of transfers to, from and within } \\
\text { Tank Farms } \\
\text { Less required contact maintenance is better, } \\
\text { etc. (rad and tox) } \\
\text { Number of active components } \\
\text { Reliability of analogous systems } \\
\text { Minimize number of support systems } \\
\text { Minimize number and frequency of PM's } \\
\text { Minimize maintenance in zone entries } \\
\text { Minimize specialized equipment and parts } \\
\text { Minimize tank entries } \\
\text { Ease of training } \\
\text { Complexity of procedures } \\
\text { Similar to other process facilities on site } \\
\text { Waste handling compatible with existing } \\
\text { systems as defined by DOE Order } 420.1 \mathrm{~B} \\
\text { Minimize operational impacts associated with } \\
\text { hazardous (generated) waste handling }\end{array}$ \\
\hline 5 & $\begin{array}{l}\text { Programmatic } \\
\text { Aspects }\end{array}$ & 20 & 5.2 & $\begin{array}{l}\text { Cost Impact } \\
\text { Schedule Impact } \\
\text { DST Space }\end{array}$ & 20 & $\begin{array}{l}5.1 .1 \\
5.1 .2 \\
5.1 .3 \\
5.2 .1 \\
5.2 .2 \\
5.2 .3 \\
5.2 .4 \\
5.3 .1 \\
5.3 .2\end{array}$ & $\begin{array}{l}\text { Capital costs } \\
\text { Life cycle costs } \\
\text { Cost profile } \\
\text { Overall schedule (confidence) } \\
\text { Licensing } \\
\text { Permitting } \\
\text { D\&D } \\
\text { How fast DST space is made available } \\
\text { Amount of DST space }\end{array}$ \\
\hline
\end{tabular}




\begin{tabular}{|c|c|c|c|c|c|c|c|}
\hline \multicolumn{8}{|c|}{ Attachment A - Decision Criteria, Measures, and Definitions For IPS Technologies } \\
\hline No. & Criteria & \begin{tabular}{|l} 
Weight \\
Factor
\end{tabular} & No. & Measures & \begin{tabular}{|l} 
Ranking \\
Factor
\end{tabular} & No. & Definitions \\
\hline & & & 5.4 & \begin{tabular}{|l|} 
Impacts to WTP and \\
Supplemental \\
Treatment, positive and \\
negative \\
\\
Impacts to other \\
facilities e.g., ETF, \\
LAB \\
Resources and materials
\end{tabular} & $\begin{array}{l}10 \\
\end{array}$ & \begin{tabular}{|l|} 
\\
5.4 .1 \\
5.4 .2 \\
5.4 .3 \\
5.4 .4 \\
5.4 .5 \\
5.4 .6 \\
5.4 .7 \\
5.4 .8 \\
\\
5.5 .1 \\
5.5 .2 \\
5.5 .3 \\
5.5 .4 \\
5.6 .1 \\
5.6 .2
\end{tabular} & $\begin{array}{l}\text { Production rate impact } \\
\text { Mission duration } \\
\text { Number of high and low level packages } \\
\text { Lessons Learned benefits for WTP } \\
\text { pretreatment } \\
\text { Technology transfer to WTP } \\
\text { ALARA } \\
\text { Diversity of technology } \\
\text { Positive programmatic impacts and } \\
\text { opportunities } \\
\text { Analytical equipment, methods, and capacity } \\
\text { Compliance to ETF WAC } \\
\text { ALARA } \\
\text { Number of Evaporator campaigns } \\
\text { Availability of Key Skills, Critical Materials, } \\
\text { Qualified Vendors } \\
\text { Stability of Critical Resource Pricing }\end{array}$ \\
\hline
\end{tabular}


RPP-RPT-37740, Rev 0

\section{ATTACHMENT B}

SOLID SEPARATION TECHNOLOGY ASSESSMENT SUMMARY MATRIX 
Attachment B - Solids Separation Technology Assessment Summary Matrix

\begin{tabular}{|c|c|c|c|}
\hline \multicolumn{4}{|c|}{ Attachment B - Solids Separation Technology Assessment Summary Matrix } \\
\hline Criteria & Measures and Definitions & Cross-flow Filtration (CFF) & Rotary Micro-filtration (RMF) \\
\hline \multirow[t]{8}{*}{$\begin{array}{l}1 \text { SAFETY } \\
1.1 \quad \text { Process Safety }\end{array}$} & $\begin{array}{l}\text { 1.1.1 Quantity of material at risk (MAR) - radiological and } \\
\text { chemical - less is better }\end{array}$ & $\begin{array}{l}\text { - The CFF system does have a larger hold up (volume) then the RMFs. } \\
\text { The quantity waste in the system is several thousand gallons. An } \\
\text { individual flush of the CFF system also would use a larger volume of } \\
\text { chemical. This technology therefore has a higher quantity of MAR. }\end{array}$ & $\begin{array}{l}\text { - Each RMF unit has a hold up of about } 40 \text { gallons or about } 160 \text { gallons } \\
\text { total for the CSSX and IX-sRF options (4 RMF units) and about } 500 \\
\text { gallons total (12 RMF units) for the FC option. Each RMF unit has less } \\
\text { MAR then the CFF unit. }\end{array}$ \\
\hline & $\begin{array}{l}\text { 1.1.2 Concentration of radiological and chemical MAR - } \\
\text { less is better }\end{array}$ & $\begin{array}{l}\text { - Since the feed vector does not have a solids component there is no } \\
\text { change in the concentration of radiological and chemical MAR. The } \\
\text { solids in the } 8 \text { feed tanks have a MAR similar to the liquid. While the } \\
\text { MAR could change with a different feed, any changes would be similar } \\
\text { for both CFF and RMF }\end{array}$ & $\begin{array}{l}\text { - Since the feed vector does not have a solids component there is no } \\
\text { change in the concentration of radiological and chemical MAR. The } \\
\text { solids in the } 8 \text { feed tanks have a MAR similar to the liquid. While the } \\
\text { MAR could change with a different feed, any changes would be similar } \\
\text { for both CFF and RMF }\end{array}$ \\
\hline & $\begin{array}{l}\text { 1.1.3 Dispersability of the MAR - less dispersible form is } \\
\text { better (e.g., solids over liquids over powders over gases) }\end{array}$ & - The MAR is a liquid with entrained solids. & - The MAR is a liquid with entrained solids.. \\
\hline & $\begin{array}{l}\text { 1.1.4 Dispersive energy, e.g., heat, off gassing, pressure, } \\
\text { etc. inherent in the process - less dispersive energy is better }\end{array}$ & $\begin{array}{l}\text { - CFF uses a high capacity recirculation pump (1,100-3,300 gpm). In the } \\
\text { event of a recirculation pump component failure (e.g. large pipe break } \\
\text { accident), dispersive energy would be significant due to the high supply } \\
\text { flow rate. }\end{array}$ & $\begin{array}{l}\text { - RMF system has significantly lower flow rates then CFF. However, the } \\
\text { rotational energy of the spinning disks will have to be evaluated as part } \\
\text { of a housing failure accident. }\end{array}$ \\
\hline & 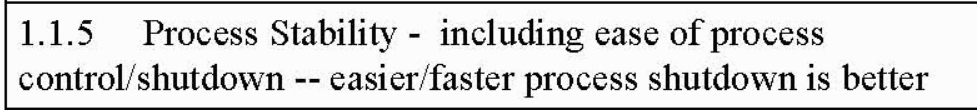 & - Quick shutdown can be achieved by shutting of the system pumps. & $\begin{array}{l}\text { - Quick shutdown can be achieved by shutting of the pumps and filter } \\
\text { motors. }\end{array}$ \\
\hline & $\begin{array}{l}\text { 1.1.6 Process that does not create a new or exacerbate an } \\
\text { existing Tank Farm hazard is preferred to one that does }\end{array}$ & $\begin{array}{l}\text { - Potential exists for one tank farm hazard accidental scenario - mixing of } \\
\text { incompatible chemicals. }\end{array}$ & $\begin{array}{l}\text { - Potential exists for one tank farm hazard accidental scenario - mixing of } \\
\text { incompatible chemicals. }\end{array}$ \\
\hline & \begin{tabular}{|ll}
1.1 .7 Less fire hazard (e.g. less quantity of combustibles, \\
including flammable gas, less flammable combustibles, etc.)
\end{tabular} & $\begin{array}{l}\text { - The CFF will have to be evaluated for flammable gas retention and } \\
\text { release due to solids in the system during shutdown and start up. The } \\
\text { solid hold up is larger in the CFF system. }\end{array}$ & $\begin{array}{l}\text { - The CFF will have to be evaluated for flammable gas retention and } \\
\text { release due to solids in the system during shutdown and start up. The } \\
\text { solid hold up is larger in the CFF system. }\end{array}$ \\
\hline & $\begin{array}{l}1.1 .8 \text { Reactive Chemicals - Process with less reactive } \\
\text { chemicals (reactivity) is better - May need to mitigate the } \\
\text { addition of nitric acid to clean RMF in tank, per JOH } \\
\text { (probably would not be done) }\end{array}$ & $\begin{array}{l}\text { - Caustic and nitric acid wash solutions are used for filter cleaning. } \\
\text { These would have to be /treated/neutralized in the CFF system to meet } \\
\text { DST specifications. }\end{array}$ & $\begin{array}{l}\text { - Caustic and nitric acid wash solutions are used for filter cleaning. Since } \\
\text { nitric acid potentially will be used for cleaning and since in two } \\
\text { configurations the RMFs are in the DST, further evaluation and changes } \\
\text { to the TF DSA may be required }\end{array}$ \\
\hline Criticality Safety & $\begin{array}{l}\text { 1.2.1 A Process that is inherently sub critical is preferred } \\
\text { over a process that relies on criticality controls }\end{array}$ & $\begin{array}{l}\text { - CFF process is sub-critical under expected conditions as it does not hold } \\
\text { up enough volume to approach the critical mass limit with the projected } \\
\text { feed vector. A review of BBI data for the source tanks also shows that } \\
\text { even if sludge was transferred the above statement is still true. } \\
\text { - Criticality is not credible because the fissile material concentration will } \\
\text { always remain too low and the neutron absorbers are too abundant. } \\
\text { - CFF does not change the result of tank farm DSA evaluation. }\end{array}$ & $\begin{array}{l}\text { - RMF process is sub-critical under expected conditions as it does not } \\
\text { hold up enough volume to approach the critical mass limit with the } \\
\text { projected feed vector A review of BBI data for the source tanks also } \\
\text { shows that even if sludge was transferred the above statement is still } \\
\text { true. } \\
\text { - Criticality is not credible because the fissile material concentration will } \\
\text { always remain too low and the neutron absorbers are too abundant. } \\
\text { - RMF does not change the result of tank farm DSA evaluation. }\end{array}$ \\
\hline $\begin{array}{ll}1.3 & \text { Industrial Safety } \\
\text { and Hygiene }\end{array}$ & $\begin{array}{l}\text { 1.3.1 Less hazards/less severe hazardous is better (e.g., less } \\
\text { hazardous chemicals, less noise, less hot surfaces, less } \\
\text { rotating equipment, less confined spaces, etc.) }\end{array}$ & $\begin{array}{l}\text { - Located in a below grade area within the IPS facility, considered to be a } \\
\text { confined space in radiation zone. } \\
\text { - Low shear high capacity circulation pump may have some noise hazards } \\
\text { with routine maintenance requirements. } \\
\text { - Does not use hazardous (severe) chemicals. }\end{array}$ & $\begin{array}{l}\text { - RMF equipment mounted at grade on an existing DST riser and a vault } \\
\text { for the FC option. } \\
\text { - Uses rotating disks (with adjustable speed) in a pressurized module. } \\
\text { - Modular design approach for filter pack. } \\
\text { - Minimal noise hazards. } \\
\text { - Does not use hazardous (severe) chemicals. }\end{array}$ \\
\hline
\end{tabular}


Attachment B - Solids Separation Technology Assessment Summary Matrix

\begin{tabular}{|c|c|c|c|}
\hline Criteria & Measures and Definitions & Cross-flow Filtration (CFF) & Rotary Micro-filtration (RMF) \\
\hline \multirow[t]{2}{*}{$\begin{array}{l}2 \text { REGULATORY / } \\
\text { STAKEHOLDER } \\
\text { ACCEPTANCE } \\
2.1 \quad \text { Achieve Tribal } \\
\text { Nations / stakeholders' } \\
\text { acceptance }\end{array}$} & 2.1.1 Early waste treatment enabled & $\begin{array}{l}\text { - Early waste pretreatment schedule can be met. Technological maturity } \\
\text { assessment is needed to confirm this. } \\
\text { - Can be permitted in } 28-33 \text { months with some additional process } \\
\text { demonstration work. }\end{array}$ & $\begin{array}{l}\text { - Early waste pretreatment schedule can be met. Technological maturity } \\
\text { assessment is needed to confirm this. } \\
\text { - Can be permitted in } 28-33 \text { months with some additional process } \\
\text { demonstration work. } \\
\text { - RMF system requires a re-design to fit into a } 42 \text {-inch diameter DST } \\
\text { riser. }\end{array}$ \\
\hline & 2.1.2 Land usage (more contaminated ground) & $\begin{array}{l}\text { - } 400-600 \mathrm{ft} 2 \text { module space will be needed within the cesium separation } \\
\text { facility depending on the processing system. }\end{array}$ & $\begin{array}{l}\text { - RMF system is mounted on a DST riser and so generally requires no } \\
\text { additional land. } \\
\text { - If FC is selected as a cesium separation technology, a } 500 \mathrm{ft} 2 \text { area will } \\
\text { be needed to house larger capacity RMF system and will be co-located } \\
\text { with the FC equipment. }\end{array}$ \\
\hline \multirow[t]{4}{*}{$\begin{array}{l}2.2 \quad \text { Achieve } \\
\text { regulators' acceptance }\end{array}$} & $\begin{array}{l}2.2 .1 \text { Compliance with applicable regulations (RCRA, } \\
\text { NEPA/SEPA, NESHAPS, NPDES, CAA, DOE Orders) }\end{array}$ & $\begin{array}{l}\text { - Depends on TC\&WM EIS and ROD completion by January } 2010 . \\
\text { - RCRA Part B Application required. } \\
\text { - Three notices of construction required. }\end{array}$ & $\begin{array}{l}\text { - Depends on TC\&WM EIS and ROD completion by January } 2010 . \\
\text { - RCRA Part B Application required. } \\
\text { - Three notices of construction required. } \\
\text { - May require } 2 \text { permitting actions, one for in-tank equipment and one for } \\
\text { equipment in IPS } \\
\text { - } 2 \text { permits required - one for IPS and one for DST modification } \\
\end{array}$ \\
\hline & 2.2.2 Impact to Disposal System Performance & $\begin{array}{l}\text { - Items such as PPEs, failed equipment, etc. are commonly disposed of } \\
\text { during Tank Farms operations. } \\
\text { - COPC are not applicable. }\end{array}$ & $\begin{array}{l}\text { - Items such as PPEs, failed equipment, etc. are commonly disposed of } \\
\text { during Tank Farms operations. } \\
\text { - COPC are not applicable. }\end{array}$ \\
\hline & 2.2.3 Secondary Waste Form and Quantity & - Does not produce secondary waste requiring new disposal form. & - Does not produce secondary waste requiring new disposal form. \\
\hline & 2.2.4 Potential impacts to other permitted facilities & $\begin{array}{l}\text { - The current WTP Project commissioning approach will support } \\
\text { commissioning and operation of the LAW processing facility, }\end{array}$ & $\begin{array}{l}\text { - The current WTP Project commissioning approach will support } \\
\text { commissioning and operation of the LAW processing facility, }\end{array}$ \\
\hline \multirow{3}{*}{$\begin{array}{l}3 \text { TECHNICAL } \\
\text { MATURITY / } \\
\text { FLEXIBILITY } \\
\text { 3.1 Technology } \\
\text { Readiness Level }\end{array}$} & 3.1.1 TRL Number & - TRL number is 3 . & - TRL number is 3 . \\
\hline & 3.1.2 Effort to mature technology (cost and schedule) & - ROM cost and schedule to mature technology is $\$ 2.0 \mathrm{M}$ over 30 months. & $\begin{array}{l}\text { - ROM cost and schedule to mature technology is } \$ 2.5 \mathrm{M} \text { over } \\
36 \text { months.(additional } 6 \text { months of time is for run-time reliability) }\end{array}$ \\
\hline & 3.1.3 Probability of Success & - Probability of success for maturing technology is "High". & - Probability of success for maturing technology is "High". \\
\hline \multirow[t]{4}{*}{$\begin{array}{l}3.2 \text { Process Flexibility } \\
\text { and robustness }\end{array}$} & 3.2.1 Ability to process a variety of feeds & $\begin{array}{l}\text { - Demonstrates "Medium" level of flexibility to process variety of } \\
\text { Hanford tank waste feeds. }\end{array}$ & $\begin{array}{l}\text { - Demonstrates "High" level of flexibility to process variety of Hanford } \\
\text { tank waste feeds. }\end{array}$ \\
\hline & 3.2.2 Ability to adjust process rate (turn up/turn down) & $\begin{array}{l}\text { - Demonstrates "High" level of flexibility to adjust process rates. } \\
\text { - Rated higher because rotational speed can also be adjusted }\end{array}$ & $\begin{array}{l}\text { - Demonstrates "High" level of flexibility to adjust process rates. } \\
\text { - Rated lower because only pump rate can be adjusted }\end{array}$ \\
\hline & 3.2.3 $\quad$ Flexibility to modify product & - "Medium" filtration product flexibility with 9 degrees of freedom. & - "Medium" filtration product flexibility with 10 degrees of freedom. \\
\hline & $\begin{array}{l}\text { 3.2.4 Ability to expand - Table this or use different basis } \\
\text { than cost for evaluating this definition. (Can larger } \\
\text { equipment, e.g., more plates in RMF, more tubes in CFF, be } \\
\text { put in same footprint?) }\end{array}$ & $\begin{array}{l}\text { - "Medium" Ability to expand with FC, "Medium" Ability to expand } \\
\text { with CSSX and "High" ability to expand with sRF ion exchange. }\end{array}$ & $\begin{array}{l}\text { - "Low" Ability to expand with FC, "Low" Ability to expand with CSSX } \\
\text { and "Low" ability to expand with sRF ion exchange. }\end{array}$ \\
\hline
\end{tabular}


Attachment B - Solids Separation Technology Assessment Summary Matrix

\begin{tabular}{|c|c|c|c|}
\hline \multirow[t]{3}{*}{ Criteria } & Measures and Definitions & \multirow{2}{*}{$\begin{array}{c}\text { Cross-flow Filtration (CFF) } \\
\text { - "Medium" flexibility to recover from out of specification product. }\end{array}$} & \multirow{2}{*}{$\begin{array}{c}\text { Rotary Micro-filtration (RMF) } \\
\text { - "Low" flexibility to recover from out of specification product. }\end{array}$} \\
\hline & 3.2.5 Recover from out of spec product & & \\
\hline & \begin{tabular}{|l} 
3.2.6 \\
projects - revisit after looking at programmatic evaluations
\end{tabular} & - "High" applicability to other DOE complex projects. & - "Medium" applicability to other DOE complex projects. \\
\hline \multirow[t]{10}{*}{$\begin{array}{l}4 \text { OPERABILITY AND } \\
\text { MAINTAINABILITY } \\
4.1 \quad \text { Ease of Process } \\
\text { control and operation }\end{array}$} & 4.1.1 Minimize number and frequency of surveillances & $\begin{array}{l}\text { - At least } 22 \text { process parameters are to be monitored and it is comparable } \\
\text { to current waste transfer operation. }\end{array}$ & $\begin{array}{l}\text { - At least } 35 \text { process parameters are to be monitored and it is comparable } \\
\text { to current waste transfer operation. } \\
\text { - With FC, a total of } 71 \text { process parameters are to be monitored due to } \\
\text { increased number of filter modules. }\end{array}$ \\
\hline & 4.1.2 Minimize number of people to operate & - Operation requires at least six people. & $\begin{array}{l}\text { - Operation requires at least five people for CSSX and IX-sRF and six } \\
\text { when used with the FC. }\end{array}$ \\
\hline & \begin{tabular}{|ll}
4.1 .3 & Ease of startup and shutdown
\end{tabular} & $\begin{array}{l}\text { - Easy to startup and shutdown based on a small number of moving } \\
\text { components (2) and a simplified process. }\end{array}$ & $\begin{array}{l}\text { - Easy to startup and shutdown based on few (6) moving components } \\
\text { when used with CSSX and IX-sRF. } \\
\text { - More complex due to higher number of moving components (14) and } \\
\text { multiple locations when used with FC. }\end{array}$ \\
\hline & 4.1.4 Minimize system complexity & $\begin{array}{l}\text { - No more complex than any of the existing waste transfer systems } \\
\text { currently used in the tank farms. }\end{array}$ & $\begin{array}{l}\text { - No more complex than any of the existing waste transfer systems } \\
\text { currently used in the tank farms. }\end{array}$ \\
\hline & 4.1.5 Minimize number of chemicals needed & $\begin{array}{l}\text { - Process uses modest quantities of basic chemicals, sodium hydroxide } \\
\text { and nitric acid. }\end{array}$ & $\begin{array}{l}\text { - Process uses modest quantities of basic chemicals, sodium hydroxide } \\
\text { and nitric acid. }\end{array}$ \\
\hline & 4.1.6 Minimize number of process and regulatory samples & $\begin{array}{l}\text { - Process sampling requirements are minimum (2) with no requirements } \\
\text { for regulatory samples. } \\
\text { - Detailed design may identify more parameters for measurements. }\end{array}$ & $\begin{array}{l}\text { - Process sampling requirements are minimum (2) with no requirements } \\
\text { for regulatory samples. } \\
\text { - Detailed design may identify more parameters for measurements. }\end{array}$ \\
\hline & \begin{tabular}{|ll}
$4.1 .7 \quad$ Batch verses continuous \\
\end{tabular} & - Routinely used as a continuous process for each campaign. & - Routinely used as a continuous process for each campaign. \\
\hline & 4.1.8 Ease of entry and exit from standby & - System shutdown and restart is easy. & - System shutdown and restart is easy. \\
\hline & 4.1.9 Wide operating margin & $\begin{array}{l}\text { - System has an acceptable operating range for the majority of Hanford } \\
\text { tank waste. }\end{array}$ & $\begin{array}{l}\text { - System has an acceptable operating range for the majority of Hanford } \\
\text { tank waste. }\end{array}$ \\
\hline & $\begin{array}{l}\text { 4.1.10 Complexity of transfers to, from and within Tank } \\
\text { Farms }\end{array}$ & $\begin{array}{l}\text { - System does not impose any complex tank waste transfer requirements } \\
\text { to the Tank Farms. }\end{array}$ & $\begin{array}{l}\text { - System does not impose any complex tank waste transfer requirements } \\
\text { to the Tank Farms. }\end{array}$ \\
\hline ALARA & 4.2.1 Less required contact maintenance is better, etc. & $\begin{array}{l}\text { - Potentially less contact maintenance is required. Most maintenance } \\
\text { activities will be done at the IPS (except for the feed pump). } \\
\text { - ALARA must be implemented during the design phase to assure } \\
\text { maximum benefits. Contaminated components will be flushed and } \\
\text { decontaminated to the extent possible prior to maintenance. } \\
\text { - Waste transfer and feed pumps, transfer piping, and filter tube bundle } \\
\text { will require contact maintenance. } \\
\text { - Design will include remote replacement and maintenance to the extent } \\
\text { possible. } \\
\text { - Instrumentation and control systems will invoke work in radiation area } \\
\text { (valve pit) or the IPS facility, but exposure can be minimized. } \\
\text { - Initial installation is in "green field" conditions }\end{array}$ & $\begin{array}{l}\text { - May require maintenance activities to be performed in a DST pit or } \\
\text { components may have to be removed from the tank for repair or } \\
\text { replacement. } \\
\text { - ALARA must be implemented during the design phase to assure } \\
\text { maximum benefits. Contaminated components will be flushed and } \\
\text { decontaminated to the extent possible prior to maintenance. } \\
\text { - Waste transfer and feed pumps, transfer line and filter assemblies will } \\
\text { require contact maintenance. } \\
\text { - Design will include remote replacement and maintenance to the extent } \\
\text { possible. } \\
\text { - Instrumentation and control systems will invoke work in radiation area } \\
\text { (valve pit) or the IPS facility, but exposure can be minimized. } \\
\text { - Replacement of seals will likely be required during 5-year mission }\end{array}$ \\
\hline Reliability & 4.3.1 Number of active components & $\begin{array}{l}\text { - Three active components (waste feed/transfer pumps, and the filter } \\
\text { back-flush system). }\end{array}$ & $\begin{array}{l}\text { - More }(6-13) \text { active components (waste feed/transfer pump motors and } \\
\text { rotary microfilter shaft motors). }\end{array}$ \\
\hline
\end{tabular}


Attachment B - Solids Separation Technology Assessment Summary Matrix

\begin{tabular}{|c|c|c|c|}
\hline \multirow[t]{2}{*}{ Criteria } & Measures and Definitions & \multirow{2}{*}{$\begin{array}{l}\text { Cross-flow Filtration (CFF) } \\
\text { - Use of large shell and tube filter systems at Hanford has not been done. } \\
\text { Single element metal filters have been used. } \\
\text { - The reliability of the CFF system should be outstanding. } \\
\text { - Single point failure as currently designed } \\
\text { - Extensive application of technology }\end{array}$} & \multirow{2}{*}{$\begin{array}{l}\text { Rotary Micro-filtration (RMF) } \\
\text { - No documented use of RMF at Hanford Site or similar system at the } \\
\text { site,. } \\
\text { - The reliability of the RMF system should be good. There is a limited } \\
\text { historical data for the reliability of the filters and rotational motor. } \\
\text { - Apparent redundancy with current design }\end{array}$} \\
\hline & 4.3.2 Reliability of analogous systems & & \\
\hline \multirow[t]{5}{*}{$\begin{array}{l}4.4 \quad \text { Ease and } \\
\text { frequency of maintenance }\end{array}$} & 4.4.1 Minimize number of support systems & $\begin{array}{l}\text { - For operation five services- power, air, water, } \mathrm{NaOH} \text { and } \mathrm{HNO} 3 \text { are } \\
\text { required. }\end{array}$ & $\begin{array}{l}\text { - For operation five services- power, air, water, } \mathrm{NaOH} \text { and } \mathrm{HNO} 3 \text { are } \\
\text { required. } \\
\text { - check need for use of air }\end{array}$ \\
\hline & $\begin{array}{l}4.4 .2 \text { Minimize number and frequency of PM's (includes } \\
\text { calibrations) }\end{array}$ & $\begin{array}{l}\text { - Preventive maintenance activities are anticipated to be routine with } \\
\text { some entry into radiation zone areas. }\end{array}$ & $\begin{array}{l}\text { - Preventive maintenance activities are anticipated to be routine with } \\
\text { some entry into radiation zone areas. Due to higher number of RM } \\
\text { units, number of activities will be higher. } \\
\text { - If used with FC, number of RMF units will be 12, increasing number of } \\
\text { maintenance activities. }\end{array}$ \\
\hline & 4.4.3 Minimize maintenance in-zone entries & $\begin{array}{l}\text { - Routine zone entry required once every five year for repair/replacement } \\
\text { of filter tube bundle. }\end{array}$ & $\begin{array}{l}\text { - Zone entry is needed every } 2 \text { years for repair/replacement of mechanical } \\
\text { seals. } \\
\text { - Frequency of replacement potentially impacted by start/stop mode of } \\
\text { operation } \\
\text { - Seal reliability impacted by planned start/stop operation. }\end{array}$ \\
\hline & 4.4.4 Minimize specialized equipment and parts & $\begin{array}{l}\text { - Crossflow filter is a commercial technology which is adapted to } \\
\text { radioactive operation. Adequate spare parts are needed. }\end{array}$ & $\begin{array}{l}\text { - Rotary microfilter assembly is specialized equipment but is based on } \\
\text { commercial technology which is modified for a radioactive operation. } \\
\text { Adequate spare parts will be needed. }\end{array}$ \\
\hline & 4.4.5 Minimize tank entries & $\begin{array}{l}\text { - Routine waste transfer through pipes per tank farm procedures requiring } \\
\text { no special DST tank entry. Installation of feed pump in the valve pit is } \\
\text { also considered a "DST tank entry". } \\
\text { - Some maintenance activities on feed pump will require a DST tank } \\
\text { entry. }\end{array}$ & $\begin{array}{l}\text { - Requires a DST entry to install filtration equipment on AP-104 riser and } \\
\text { some maintenance activities. }\end{array}$ \\
\hline \multirow[t]{3}{*}{$\begin{array}{lr}4.5 \quad \text { Ease of } \\
\text { Implementation }\end{array}$} & 4.5.1 Ease of training & $\begin{array}{l}\text { - CFF is a passive unit with an uncomplicated operating principles } \\
\text { leading to non-complex training needs. }\end{array}$ & $\begin{array}{l}\text { - RMF is a compact design with basic control and configured for ease of } \\
\text { assembly and maintenance, eliminating complex training needs. }\end{array}$ \\
\hline & 4.5.2 Complexity of procedures & - No complexity for O\&M procedure is anticipated. & - No complexity for O\&M procedure is anticipated. \\
\hline & $\begin{array}{|ll|}\text { 4.5.3 } & \text { Similar to other process facilities on Hanford site }\end{array}$ & - Similar technology is not in use at Hanford Site & - Similar technology is not in use at Hanford Site \\
\hline \multirow[t]{2}{*}{$\begin{array}{l}4.6 \quad \text { Liquid/solid } \\
\text { secondary waste }\end{array}$} & $\begin{array}{l}\text { 4.6.1 Waste handling compatible with existing systems as } \\
\text { defined by DOE Order 420.1B - check reference to DOE } \\
\text { Order with Grigsby }\end{array}$ & - CFF technology is compatible with DOE Order 420.1B. & - RMF technology is compatible with DOE Order 420.1B. \\
\hline & $\begin{array}{l}\text { 4.6.2 Minimize operational impacts associated with } \\
\text { hazardous (generated) waste handling }\end{array}$ & $\begin{array}{l}\text { - The technology is suited for continuous normal operation, with no } \\
\text { impact for handling of secondary waste. } \\
\text { - Operation requires suspension once in } 5 \text { yr for replacement of filter tube } \\
\text { bundles and disposal of failed equipment. }\end{array}$ & $\begin{array}{l}\text { - The technology is suited for continuous normal operation, with no } \\
\text { impact for handling of secondary waste. } \\
\text { - Operation requires suspension every } 2 \text { year for replacement of } \\
\text { mechanical seals and disposal of failed equipment. }\end{array}$ \\
\hline $\begin{array}{l}5 \text { PROGRAMMATIC } \\
\text { ASPECTS } \\
5.1 \quad \text { Cost Impact }\end{array}$ & 5.1.1 Capital costs (for comparative purposes only) & $\begin{array}{l}\text { - With expected accuracy range of }-30 \% \text { to }+50 \% \text { : CFF }- \text { FC capital costs } \\
=\$ 64 \mathrm{M} \text { to } \$ 140 \mathrm{M} \text {, CFF - CSSX capital costs }=\$ 82 \mathrm{M} \text { to } \$ 180 \mathrm{M} \text {, CFF - } \\
\text { IX capital costs }=\$ 54 \mathrm{M} \text { to } \$ 120 \mathrm{M} \text {. }\end{array}$ & $\begin{array}{l}\text { - With expected accuracy range of }-30 \% \text { to }+50 \%: \text { RMF }- \text { FC capital } \\
\text { costs }=\$ 75 \mathrm{M} \text { to } \$ 160 \mathrm{M}, \mathrm{RMF}-\mathrm{CSSX} \text { capital costs }=\$ 87 \mathrm{M} \text { to } \$ 190 \mathrm{M} \text {, } \\
\mathrm{RMF}-\mathrm{IX} \text { capital costs }=\$ 59 \mathrm{M} \text { to } \$ 130 \mathrm{M} \text {. }\end{array}$ \\
\hline
\end{tabular}


Attachment B - Solids Separation Technology Assessment Summary Matrix

\begin{tabular}{|c|c|c|c|}
\hline \multicolumn{4}{|c|}{ Attachment B - Solids Separation Technology Assessment Summary Matrix } \\
\hline \multirow[t]{3}{*}{ Criteria } & Measures and Definitions & \multirow{2}{*}{$\begin{array}{l}\text { Cross-flow Filtration (CFF) } \\
\text { - With expected accuracy range of }-30 \% \text { to }+50 \% \text { (applied to capital cost } \\
\text { contribution): CFF - FC life cycle costs (w/o T\&Rs) } \$ 170 \mathrm{M} \text { to } \\
\$ 240 \mathrm{M}, \mathrm{CFF}-\text { CSSX life cycle costs (w/evap) }=\$ 190 \mathrm{M} \text { to } \$ 290 \mathrm{M}, \mathrm{CFF} \\
\text { - IX life cycle costs }=\$ 140 \mathrm{M} \text { to } \$ 200 \mathrm{M} \text {. }\end{array}$} & \multirow{2}{*}{$\begin{array}{l}\text { Rotary Micro-filtration (RMF) } \\
\text { - With expected accuracy range of }-30 \% \text { to }+50 \% \text { (applied to capital cost } \\
\text { contribution): RMF - FC life cycle costs (w/o T\&Rs) }=\$ 190 \mathrm{M} \text { to } \\
\$ 280 \mathrm{M}, \mathrm{RMF}-\mathrm{CSSX} \text { life cycle costs }(\mathrm{W} / \mathrm{evap})=\$ 210 \mathrm{M} \text { to } \$ 310 \mathrm{M}, \\
\text { RMF - IX life cycle costs }=\$ 150 \mathrm{M} \text { to } \$ 220 \mathrm{M} \text {. }\end{array}$} \\
\hline & 5.1.2 Life cycle costs (for comparative purposes only) & & \\
\hline & 5.1.3 Cost profile (for comparative purposes only) & $\begin{array}{l}\text { - Both the lowest capital cost and the lowest life cycle cost result from } \\
\text { pairing CFF with IX-sRF. (for comparative purposes only) }\end{array}$ & $\begin{array}{l}\text { - Both the highest capital cost and highest life cycle cost result from } \\
\text { pairing RMF with CSSX. (for comparative purposes only) }\end{array}$ \\
\hline \multirow[t]{4}{*}{ 5.2 Schedule Impact } & $\begin{array}{l}\text { 5.2.1 Overall schedule (confidence) - for comparative } \\
\text { purposes only }\end{array}$ & $\begin{array}{l}\text { - Schedule estimate for both filtration technologies were deemed } \\
\text { sufficiently similar. }\end{array}$ & $\begin{array}{l}\text { - Schedule estimate for both filtration technologies were deemed } \\
\text { sufficiently similar. }\end{array}$ \\
\hline & 5.2.2 Licensing & $\begin{array}{l}\text { - Due to considerable experience with this technology at various DOE } \\
\text { sites (including WTP), its safety analysis should not impact overall } \\
\text { schedule. }\end{array}$ & $\begin{array}{l}\text { - Due to lack of enough design and process information, safety analysis } \\
\text { will require additional efforts and time. }\end{array}$ \\
\hline & 5.2.3 Permitting & $\begin{array}{l}\text { - Does not impact RCRA part B permitting schedule as it is the first step } \\
\text { of the total pretreatment process and does not produce any secondary } \\
\text { waste. }\end{array}$ & $\begin{array}{l}\text { - Does not impact RCRA part B permitting schedule as it is the first step } \\
\text { of the total pretreatment process and does not produce any secondary } \\
\text { waste. }\end{array}$ \\
\hline & 5.2.4 D\&D & $\begin{array}{l}\text { - Considerations for D\&D will be accommodated during the IPS design. } \\
\text { Negligible impact on IPS schedule. }\end{array}$ & $\begin{array}{l}\text { - Considerations for D\&D will be accommodated during the IPS design. } \\
\text { Negligible impact on IPS schedule. }\end{array}$ \\
\hline \multirow[t]{2}{*}{$\begin{array}{ll}5.3 \quad \text { DST Space } \\
\end{array}$} & 5.3.1 How fast DST space is made available $-\mathrm{N} / \mathrm{A}$ & - Does not directly impact rate of freed up DST space. & - Does not directly impact rate of freed up DST space. \\
\hline & 5.3.2 Amount of DST space $-\mathrm{N} / \mathrm{A}$ & $\begin{array}{l}\text { - Generates a total of } 200,000 \mathrm{gals} \text { of } 20 \mathrm{wt} \% \text { entrained solids waiting to } \\
\text { be processed as } \mathrm{HLW} \text { feed. } \\
\text { - Larger CFF system volume and higher filter cleaning frequency will } \\
\text { generate neutralized wash solution volumes significantly (10+ times) } \\
\text { greater than RMF. }\end{array}$ & $\begin{array}{l}\text { - Generates a total of } 200,000 \text { gals of } 20 \% \text { entrained solids waiting to be } \\
\text { processed as HLW feed. } \\
\text { - Smaller RMF system volume and lower filter cleaning frequency will } \\
\text { generate neutralized wash solution volumes significantly less than CFF. } \\
\text { - Neutralized wash solution volumes for FC will be } \sim 2-3 \text { times than those } \\
\text { for CSSX or IX-sRF due to increased number of filters. }\end{array}$ \\
\hline \multirow{8}{*}{$\begin{array}{l}5.4 \quad \text { Impacts to WTP } \\
\text { and Supplemental } \\
\text { Treatment, positive and } \\
\text { negative }\end{array}$} & 5.4.1 Production rate impact $-\mathrm{N} / \mathrm{A}$ & $\begin{array}{l}\text { - No production rate impact to WTP (HLW and LAW) or supplemental } \\
\text { Treatment Plant. }\end{array}$ & $\begin{array}{l}\text { - No production rate impact to WTP (HLW and LAW) or supplemental } \\
\text { Treatment Plant. }\end{array}$ \\
\hline & 5.4.2 Mission duration $-\mathrm{N} / \mathrm{A}$ & - No impact to overall WTP primary mission duration. & - No impact to overall WTP primary mission duration. \\
\hline & 5.4.3 Number of high and low level packages $-\mathrm{N} / \mathrm{A}$ & $\begin{array}{l}\text { - No impact on high or low level waste packages to be produced by WTP } \\
\text { or supplemental treatment plants. }\end{array}$ & $\begin{array}{l}\text { - No impact on high or low level waste packages to be produced by WTP } \\
\text { or supplemental treatment plants. }\end{array}$ \\
\hline & 5.4.4 Lessons Learned benefits for WTP pretreatment & $\begin{array}{l}\text { - IPS CFF deployment, start up and operational experience will provide } \\
\text { lessons learned feedback to the WTP pretreatment facility. }\end{array}$ & - RMF process does not provide lessons learned for WTP. \\
\hline & 5.4.5 Technology transfer to WTP & - Does not provide technology transfer for WTP. & $\begin{array}{l}\text { - RMF can provide technology transfer to WTP if for any reason CFF did } \\
\text { not perform in the WTP. RMF can also provide filtration support to } \\
\text { WTP if needed. }\end{array}$ \\
\hline & 5.4.6 ALARA - N/A & - No ALARA impact to WTP. & - No ALARA impact to WTP. \\
\hline & 5.4.7 Diversity of technology & - CFF does not provide technology diversity. & $\begin{array}{l}\text { - RMF provides technology diversity for waste filtration needs at } \\
\text { Hanford. This technology can be adapted to other tanks in tank farms. }\end{array}$ \\
\hline & 5.4.8 Positive programmatic impacts and opportunities & $\begin{array}{l}\text { - Provide potential cost reduction through shared development costs and } \\
\text { reduced WTP startup costs and reduces technical and schedule risk } \\
\text { through lessons learned. }\end{array}$ & - No programmatic opportunities are identified yet. \\
\hline
\end{tabular}


Attachment B - Solids Separation Technology Assessment Summary Matrix

\begin{tabular}{|c|c|c|c|}
\hline \multicolumn{4}{|c|}{ Attachment B - Solids Separation Technology Assessment Summary Matrix } \\
\hline Criteria & Measures and Definitions & Cross-flow Filtration (CFF) & Rotary Micro-filtration (RMF) \\
\hline \multirow[t]{4}{*}{$\begin{array}{l}5.5 \quad \text { Impacts to other } \\
\text { facilities e.g., ETF, LAB }\end{array}$} & 5.5.1 Analytical equipment, methods, and capacity $-\mathrm{N} / \mathrm{A}$ & $\begin{array}{l}\text { - No impact to analytical laboratories (in-line measurement of solids } \\
\text { concentration). }\end{array}$ & $\begin{array}{l}\text { - No impact to analytical laboratories (solids are returned directly to feed } \\
\text { tank AP-104). }\end{array}$ \\
\hline & 5.5.2 Compliance to ETF WAC - N/A & - Does not impact ETF operation. & - Does not impact ETF operation. \\
\hline & 5.5.3 ALARA & $\begin{array}{l}\text { - Because CFF included as part of new IPS facility, ALARA will be more } \\
\text { easily incorporated into its process design. } \\
\text { - Initial installation is under "green field" conditions }\end{array}$ & $\begin{array}{l}\text { - Because RMF is being installed in an existing DST, ALARA will be } \\
\text { more difficult to incorporate into its process design. }\end{array}$ \\
\hline & 5.5.4 Number of Evaporator campaigns - N/A & - Does not directly impact 242-A Evaporator campaigns. & - Does not directly impact 242-A Evaporator campaigns. \\
\hline \multirow[t]{2}{*}{$\begin{array}{l}5.6 \text { Resources and } \\
\text { materials }\end{array}$} & $\begin{array}{l}\text { 5.6.1 Availability of Key Skills, Critical Materials, } \\
\text { Qualified Vendors }\end{array}$ & $\begin{array}{l}\text { - Crossflow filtration unit can be assembled by multiple experienced } \\
\text { vendors with limited technical oversight. } \\
\text { - CFF requires very high capacity recirculation pump. } \\
\text { - German supplier provides best quality material (single source?) }\end{array}$ & $\begin{array}{l}\text { - RMF will require some technological development resources to support } \\
\text { re-engineering of the system for Hanford tank specific design. } \\
\text { - Only a single vendor has been identified who is capable of assembling } \\
\text { these modules and the vendor will require strong participation of } \\
\text { technology experts from the DOE sites. }\end{array}$ \\
\hline & 5.6.2 Stability of Critical Resource Pricing & $\begin{array}{l}\text { - Does not require any special or unusual material of construction. } \\
\text { - No specific critical material pricing risk is anticipated. }\end{array}$ & $\begin{array}{l}\text { - Does not require any special or unusual material of construction. } \\
\text { - Due to a single source vendor, may be subjected to some pricing risk. }\end{array}$ \\
\hline
\end{tabular}


RPP-RPT-37740, Rev 0

\section{ATTACHMENT C}

\section{CESIUM SEPARATION TECHNOLOGY ASSESSMENT SUMMARY MATRIX}




\begin{tabular}{|c|c|c|c|c|}
\hline \multicolumn{5}{|c|}{ Attachment C-Cesium Separation Technology Assessment Summary Matrix } \\
\hline Criteria & Measures and Definitions & Fractional Crystallization $(\mathrm{FC})$ & Caustic-Side Solvent Extraction (CSSX) & Ion Exchange with sRF (IX-sRF) \\
\hline \multirow[t]{7}{*}{$\begin{array}{l}\text { 1 SAFETY } \\
1.1 \quad \text { Process Safety }\end{array}$} & $\begin{array}{l}\text { 1.1.1 Quantity of material at risk (MAR) - } \\
\text { radiological and chemical - less is better }\end{array}$ & $\begin{array}{l}\text { - The radionuclide (Ci) MAR quantity ranges are } \\
\mathrm{E}+04 \text { to } \mathrm{E}+05 \text { for feed receipt tank. Since FC has } \\
\text { the larger feed tank it has larger quantity of MAR. } \\
\text { - The radionuclide (Ci) MAR quantity ranges are } \\
\mathrm{E}+04 \text { to } \mathrm{E}+05 \text { for Cs Product tank. } \\
\text { - The radionuclide (Ci) MAR quantity ranges are } \\
8.65 \mathrm{E}+01 \text { to } 4.46 \mathrm{E}+02 \text { for LAW Product tank. } \\
\text { - Toxicological doses are driven by the chemicals in } \\
\text { the DST wastes. }\end{array}$ & $\begin{array}{l}\text { - The radionuclide (Ci) MAR quantity ranges are } \\
\text { E+04 to E+05 for feed receipt tank. } \\
\text { - The radionuclide (Ci) MAR quantity ranges are } \\
\text { E+04 for Cs Product tank. } \\
\text { - The LAW Product tank MAR is similar to the IX } \\
\text { technology. } \\
\text { - Toxicological doses are driven by the chemicals in } \\
\text { the DST wastes and not process chemicals. }\end{array}$ & $\begin{array}{l}\text { - The radionuclide (Ci) MAR quantity ranges are } \\
\mathrm{E}+04 \text { to } \mathrm{E}+05 \text { for feed receipt tank. } \\
\text { - The radionuclide (Ci) MAR quantity ranges are } \\
\mathrm{E}+04 \text { for } \mathrm{Cs} \text { Product tank. } \\
\text { - The radionuclide (Ci) MAR quantity ranges are } \\
2.17 \mathrm{E}+02 \text { to } 1.15 \mathrm{E}+03 \text { for LAW Product tank. } \\
\text { - Toxicological doses are driven by the chemicals in } \\
\text { the DST wastes and not process chemicals. }\end{array}$ \\
\hline & $\begin{array}{l}\text { 1.1.2 Concentration of radiological and } \\
\text { chemical MAR - less is better - focused on } \\
\text { toxilogical over radiological }\end{array}$ & $\begin{array}{l}\text { - ULDs of the LAW ( } 0.5 \text { to } 2.8) \text { to the WTP are } \\
\text { very low (similar for all three technologies). } \\
\text { ULDs range for Cs return stream is } 99.2 \text { to } 515.6 \text {. }\end{array}$ & $\begin{array}{l}\text { - ULDs of the LAW (1.3 to } 6.7) \text { to the WTP are } \\
\text { very low. ULDs range for Cs return stream is } 33.2 \\
\text { to } 278.2 \text {. The strip solution has ULD values up to } \\
700 \text {. }\end{array}$ & $\begin{array}{l}\text { - ULDs of the LAW ( } 1.3 \text { to } 6.7) \text { to the WTP are } \\
\text { very low. ULDs range for Cs return stream is } \\
155.4 \text { to } 342.0 \text {. }\end{array}$ \\
\hline & $\begin{array}{l}\text { 1.1.3 Dispersability of the MAR - less } \\
\text { dispersible form is better (e.g., solids over } \\
\text { liquids over powders over gases) }\end{array}$ & $\begin{array}{l}\text { - Minimal dispersability as MAR remains in a liquid } \\
\text { phase. } \\
\text { - Some of the MAR can be entrained in the vapor } \\
\text { phase. }\end{array}$ & $\begin{array}{l}\text { - Minimal dispersability as MAR always remains in } \\
\text { a liquid phase in non-fire type accidents. } \\
\text { - The MAR is mixed with the solvent. }\end{array}$ & $\begin{array}{l}\text { - Minimal dispersability as MAR remains in a liquid } \\
\text { phase. } \\
\text { - Dispersability of MAR for solid phase (resin } \\
\text { column) could be problematic in a fire accident } \\
\text { when IX column is loaded with Cs }\end{array}$ \\
\hline & $\begin{array}{l}\text { 1.1.4 Dispersive energy, e.g., heat, off } \\
\text { gassing, pressure, etc. inherent in the process } \\
\text { - less dispersive energy is better }\end{array}$ & $\begin{array}{l}\text { - Dispersion energy comes mainly from kinetic } \\
\text { energy from transfer pumps and process steam and } \\
\text { gravitational energy from tanks and vessels. } \\
\text { - The FC recirculation pumps are rated for over } \\
5000 \text { gpm. } \\
\text { - Centrifuge needs to be factored in here }(1200 \mathrm{rpm})\end{array}$ & $\begin{array}{l}\text { Dispersive energy comes from rotational kinetic } \\
\text { energy, reactive chemical energy, organic solvents } \\
\text { (fires), and gravitational energy from tanks and } \\
\text { vessels. }\end{array}$ & $\begin{array}{l}\text { Dispersive energy can be kinetic energy (pumps), } \\
\text { reactive chemical energy, H2 gassing, and } \\
\text { gravitational energy from tanks and vessels. } \\
\text { - Decay heat on resin column accounted for in } 1.1 .7\end{array}$ \\
\hline & $\begin{array}{l}\text { 1.1.5 Process Stability - including ease of } \\
\text { process control/shutdown -- easier/faster } \\
\text { process shutdown is better }\end{array}$ & $\begin{array}{l}\text { - The FC does operate at a slightly evaluated } \\
\text { temperature. Boiling can be easy stopped by } \\
\text { reducing the vacuum. Long term shut down } \\
\text { requires dumping or flushing of the solution to } \\
\text { minimize further solids formation. }\end{array}$ & $\begin{array}{l}\text { - Turning off the pumps and contactors achieves a } \\
\text { quick shutdown. But stripping of the Cs from the } \\
\text { organic would take several hours for a long term } \\
\text { shutdown. } \\
\text { - Stripping of Cs may need to be performed as part } \\
\text { of a shutdown operation }\end{array}$ & $\begin{array}{l}\text { - Simple turning off the pumps achieves the quick } \\
\text { shutdown, but rise and elution of } 137 \mathrm{Cs} \text { from resin } \\
\text { columns maybe required for long term shutdown } \\
\text { - Elution of Cs may need to be performed as part of } \\
\text { a shutdown operation }\end{array}$ \\
\hline & $\begin{array}{l}\text { 1.1.6 Process that does not create a new or } \\
\text { exacerbate an existing Tank Farm hazard is } \\
\text { preferred to one that does }\end{array}$ & $\begin{array}{l}\text { - The potential for solids in the FC return stream (or } \\
\text { in the tank as the solution cools) will need further } \\
\text { evaluation. No other potential were identified } \\
\text { which exacerbate tank farm hazard accidental } \\
\text { scenarios. }\end{array}$ & $\begin{array}{l}\text { - Potential exists for one tank farm hazard accidental } \\
\text { scenarios - mixing of incompatible chemicals. }\end{array}$ & $\begin{array}{l}\text { - Potential exists for one tank farm hazard accidental } \\
\text { scenarios - mixing of incompatible chemicals. }\end{array}$ \\
\hline & $\begin{array}{l}\text { 1.1.7 Less fire hazard (e.g. less quantity of } \\
\text { combustibles, including flammable gas, less } \\
\text { flammable combustibles, etc.) }\end{array}$ & $\begin{array}{l}\text { - Does not use combustible material. } \\
\text { - Elevated temperature can increase flammable gas } \\
\text { due to radiolysis and chemical reactions }\end{array}$ & $\begin{array}{l}\text { - Use of organic solvents with a low flash point of } \\
62^{\circ} \mathrm{C} \text { (Isopar L) elevates fire safety concern. } \\
\text { - Organic susceptible to forming hydrogen gas due } \\
\text { to radiolysis - postulated accident }\end{array}$ & $\begin{array}{l}\text { - Process is susceptible to forming hydrogen gas due } \\
\text { to radiolysis. } \\
\text { - Various chemicals used in the process may } \\
\text { generate heat if mixed improperly. } \\
\text { - Decay heat on resin column increases potential for } \\
\text { fire, but water-cooled jacket will be integrated into } \\
\text { IX column design }\end{array}$ \\
\hline
\end{tabular}




\begin{tabular}{|c|c|c|c|c|}
\hline \multicolumn{5}{|c|}{ Attachment C-Cesium Separation Technology Assessment Summary Matrix } \\
\hline Criteria & Measures and Definitions & Fractional Crystallization (FC) & Caustic-Side Solvent Extraction (CSSX) & Ion Exchange with sRF (IX-sRF) \\
\hline & $\begin{array}{l}\text { 1.1.8 Reactive Chemicals - Process with } \\
\text { less reactive chemicals (reactivity) is better }\end{array}$ & $\begin{array}{l}\text { - Only chemical that may be used is nitric acid (low } \\
\text { concentration) for cleaning. } \\
\text { - Lowest chemical reactivity. }\end{array}$ & $\begin{array}{l}\text { - Uses caustic, nitric acid and } 4 \text { different organic } \\
\text { solvents components. Organic solvents have } \\
\text { negligible or no chemical reactivity. } \\
\text { - Interactions of nitric acid with caustic solutions } \\
\text { and strong acids with organic solvents should be } \\
\text { carefully monitored and controlled. } \\
\text { - More concentration and higher quantity of } \\
\text { hazardous chemicals, e.g., nitric acid in IPS }\end{array}$ & $\begin{array}{l}\text { - Uses caustic, nitric acid and sRF organic resins. } \\
\text { - Interactions of nitric acid with caustic solutions } \\
\text { should be carefully monitored and controlled. } \\
\text { - More concentration and higher quantity of } \\
\text { hazardous chemicals, e.g., nitric acid in IPS }\end{array}$ \\
\hline $1.2 \quad$ Criticality Safety & $\begin{array}{l}\text { 1.2.1 A Process that is inherently sub } \\
\text { critical is preferred over a process that relies } \\
\text { on criticality controls }\end{array}$ & $\begin{array}{l}\text { - FC process is sub-critical under expected } \\
\text { conditions as it does not hold up enough feed } \\
\text { volume to approach the critical mass limit in any } \\
\text { condition. } \\
\text { - FC does not change the result of tank farm DSA } \\
\text { evaluation. }\end{array}$ & $\begin{array}{l}\text { - CSSX process is sub-critical under expected } \\
\text { conditions as it does not hold up enough feed } \\
\text { volume to approach the critical mass limit in any } \\
\text { condition. } \\
\text { - CSSX does not change the result of tank farm } \\
\text { DSA evaluation. }\end{array}$ & $\begin{array}{l}\text { - IX-sRF process is sub-critical under expected } \\
\text { conditions as it does not hold up enough feed } \\
\text { volume to approach the critical mass limit in any } \\
\text { condition. } \\
\text { - IX-sRF does not change the result of tank farm } \\
\text { DSA evaluation. }\end{array}$ \\
\hline $\begin{array}{l}1.3 \text { Industrial Safety } \\
\text { and Hygiene }\end{array}$ & $\begin{array}{l}\text { 1.3.1 Less hazards/less severe hazardous is } \\
\text { better (e.g., less hazardous chemicals, less } \\
\text { noise, less hot surfaces, less rotating } \\
\text { equipment, less confined spaces, etc.) }\end{array}$ & $\begin{array}{l}\text { - Large product tanks }(8,600-32,000 \text { gals) along } \\
\text { with pumps, heat exchangers, reboilers, and } \\
\text { condensers are located in below-grade areas of } \\
\text { confined space in a radiation zone. } \\
\text { - Two crystallizers are tall }(30 \mathrm{ft}) \text { requiring a ladder } \\
\text { and elevated walkways for access. } \\
\text { - Does not add chemicals for processing. }\end{array}$ & $\begin{array}{l}\text { - Large product tanks }(11,500-45,000 \text { gas) along } \\
\text { with contactors and associated pumps and heat } \\
\text { exchangers are located below grade areas of } \\
\text { confined space in a radiation zone. } \\
\text { - Uses organic solvent, } \mathrm{NaOH}, \mathrm{NaNO} 2 \text { and } \mathrm{HNO} 3 \\
\text { requiring chemical area and handling. }\end{array}$ & $\begin{array}{l}\text { - Large product tanks ( } 11,500-45,000 \text { gas) along } \\
\text { with contactors and associated pumps and heat } \\
\text { exchangers are located below grade areas of } \\
\text { confined space in a radiation zone. } \\
\text { - Uses ion exchange resin, } \mathrm{NaOH}, \mathrm{NaNO} 2 \text { and } \\
\text { HNO3 requiring chemical area and handling. }\end{array}$ \\
\hline \multirow[t]{2}{*}{$\begin{array}{l}2 \text { REGULATORY / } \\
\text { STAKEHOLDER } \\
\text { ACCEPTANCE } \\
2.1 \quad \text { Achieve Tribal } \\
\text { Nations / stakeholders' } \\
\text { acceptance }\end{array}$} & 2.1.1 Early waste treatment enabled & $\begin{array}{l}\text { - Early waste pretreatment schedule expectation can } \\
\text { be met. Technological maturity assessment is } \\
\text { needed to confirm this. } \\
\text { - Some additional demonstration and verification } \\
\text { work is required for Hanford use. } \\
\text { - Based on familiarity with evaporator technology - } \\
\text { stakeholders are less likely to support } \\
\text { implementation of a somewhat analogous } \\
\text { technology }\end{array}$ & $\begin{array}{l}\text { - Early waste pretreatment schedule expectation can } \\
\text { be met. Technological maturity assessment is } \\
\text { needed to confirm this. } \\
\text { - Some additional demonstration and verification } \\
\text { work is required for Hanford use. } \\
\text { - New technology to Hanford - stakeholders are } \\
\text { least likely to support implementation of a less } \\
\text { familiar technology }\end{array}$ & $\begin{array}{l}\text { - Early waste pretreatment schedule expectation can } \\
\text { be met. Technological maturity assessment is } \\
\text { needed to confirm this. } \\
\text { - Limited demonstration and verification work is } \\
\text { required. } \\
\text { - Based on familiarity with past Hanford separations } \\
\text { and WTP based technology -stakeholders are most } \\
\text { likely to support implementation of a more } \\
\text { familiar technology }\end{array}$ \\
\hline & $\begin{array}{l}2.1 .2 \text { Land usage (more contaminated } \\
\text { ground) }\end{array}$ & $\begin{array}{l}\text { - Process Vault/Building footprint is } 5699 \mathrm{ft} 2 \text { (with } \\
\text { RMF) and } 5963 \mathrm{ft} 2 \text { (with CFF). }\end{array}$ & $\begin{array}{l}\text { - Process Vault/Building footprint is } 6016 \mathrm{ft} 2 \text { (with } \\
\text { RMF) and } 6628 \mathrm{ft} 2 \text { (with CFF). }\end{array}$ & $\begin{array}{l}\text { - Process Vault/Building footprint is } 4032 \mathrm{ft} 2 \text { (with } \\
\mathrm{RMF} \text { ) and } 4610 \mathrm{ft} 2 \text { (with CFF). }\end{array}$ \\
\hline $\begin{array}{l}2.2 \quad \text { Achieve } \\
\text { regulators' acceptance }\end{array}$ & $\begin{array}{l}2.2 .1 \text { Compliance with applicable } \\
\text { regulations (RCRA, NEPA/SEPA, } \\
\text { NESHAPS, NPDES, CAA, DOE Orders) }\end{array}$ & $\begin{array}{l}\text { - Can be permitted in } 28-33 \text { months with some } \\
\text { additional process demonstration work. } \\
\text { - Depends on TC\&WM EIS and ROD being } \\
\text { completed by January } 2010 \text {. } \\
\text { - RCRA Part B Application required. } \\
\text { - Three notices of construction required. }\end{array}$ & $\begin{array}{l}\text { - Can be permitted in } 28 \text { - } 33 \text { months with some } \\
\text { additional process demonstration work. } \\
\text { - Depends on TC\& WM EIS and ROD being } \\
\text { completed by January } 2010 \text {. } \\
\text { - RCRA Part B Application required. } \\
\text { - Three notices of construction required. }\end{array}$ & $\begin{array}{l}\text { - Can be permitted in } 28 \text { - } 33 \text { months with some } \\
\text { additional process demonstration work. } \\
\text { - Depends on TC\&WM EIS and ROD being } \\
\text { completed by January } 2010 \text {. } \\
\text { - RCRA Part B Application required } \\
\text { - Three notices of construction required. }\end{array}$ \\
\hline
\end{tabular}




\begin{tabular}{|c|c|c|c|c|}
\hline \multicolumn{5}{|c|}{ Attachment C - Cesium Separation Technology Assessment Summary Matrix } \\
\hline Criteria & Measures and Definitions & Fractional Crystallization $(\mathrm{FC})$ & Caustic-Side Solvent Extraction (CSSX) & Ion Exchange with sRF (IX-sRF) \\
\hline & $\begin{array}{l}\text { 2.2.2 Impact to Disposal System } \\
\text { Performance }\end{array}$ & $\begin{array}{l}\text { - Further investigation of potential COPC concern } \\
\text { for secondary waste disposal system is required. } \\
\text { - The current WTP Project commissioning approach } \\
\text { will support commissioning and operation of the } \\
\text { LAW processing facility, without the support of } \\
\text { the Pretreatment facility. } \\
\text { - FC will remove Tc from LAW feed; a substantial } \\
\text { positive for FC }\end{array}$ & $\begin{array}{l}\text { - Further investigation of potential COPC concern } \\
\text { for secondary waste disposal system is required. } \\
\text { - The current WTP Project commissioning approach } \\
\text { will support commissioning and operation of the } \\
\text { LAW processing facility, without the support of } \\
\text { the Pretreatment facility. }\end{array}$ & $\begin{array}{l}\text { - Further investigation of potential COPC concern } \\
\text { for secondary waste disposal system is required. } \\
\text { - The current WTP Project commissioning approach } \\
\text { will support commissioning and operation of the } \\
\text { LAW processing facility, without the support of } \\
\text { the Pretreatment facility. }\end{array}$ \\
\hline & 2.2.3 Secondary Waste Form and Quantity & $\begin{array}{l}\text { - Generates 7,256,143 L (1.9 million gallons) of } \\
\text { liquid effluent for disposal at ETF. }\end{array}$ & $\begin{array}{l}\text { - Generates } 680-1,475 \mathrm{~L} \text { (180-390 gallons) of } \\
\text { liquid organic solvent per year for disposal. }\end{array}$ & $\begin{array}{l}\text { - Generates } 4,535 \mathrm{~kg}(10,000 \mathrm{lbs}) \text { of spent resin for } \\
\text { disposal. }\end{array}$ \\
\hline & $\begin{array}{l}\text { 2.2.4 Potential impacts to other permitted } \\
\text { facilities }\end{array}$ & $\begin{array}{l}\text { - Secondary waste to ETF will meet Waste } \\
\text { Acceptance Criteria. } \\
\text { - Permit modification for IDF will be needed sooner } \\
\text { than anticipated (allows disposal of LAW glass) }\end{array}$ & $\begin{array}{l}\text { - Does not generate secondary waste for ETF. } \\
\text { - Permafix is the permitted facility for disposal of } \\
\text { organics - is it on their list? Possible need for } \\
\text { modified permit } \\
\text { - Trace amounts of organics to DSTs; waste codes } \\
\text { going forward to WTP? } \\
\text { - Permit modification for IDF will be needed sooner } \\
\text { than anticipated (allows disposal of LAW glass) }\end{array}$ & $\begin{array}{l}\text { - Does not generate secondary waste for ETF. } \\
\text { - Permit mod for IDF to accept spent resins } \\
\text { - Permit modification for IDF will be needed sooner } \\
\text { than anticipated (allows disposal of LAW glass) }\end{array}$ \\
\hline \multirow[t]{3}{*}{$\begin{array}{l}3 \text { TECHNICAL } \\
\text { MATURITY / } \\
\text { FLEXIBILITY } \\
3.1 \quad \text { Technology } \\
\text { Readiness Level }\end{array}$} & 3.1.1 TRL Number & $\begin{array}{l}\text { - TRL number is } 4 \text { - this TRL number is based on } \\
\text { the technical and programmatic work that was } \\
\text { done in support of SST wastes in } 200 \mathrm{~W} \text {. May } \\
2008 \text { review of original assessment found that } \\
\text { original work was sufficiently valid to maintain } \\
\text { TRL \# of } 4 \text {. } \\
\text { - Original assessment was based on SST saltcake } \\
\text { feeds, rather than DST supernate feeds identified } \\
\text { for IPS }\end{array}$ & $\begin{array}{l}\text { - TRL number is } 3 \\
\text { - Lab testing with simulants and modeling needed } \\
\text { - Potassium could be an issue }\end{array}$ & $\begin{array}{l}\text { - TRL number is } 3 \\
\text { - Cs separation demonstrated with real waste } \\
\text { - Equipment optimization is primarily need } \\
\text { (engineering, not development) }\end{array}$ \\
\hline & $\begin{array}{l}\begin{array}{l}\text { 3.1.2 Effort to mature technology (cost and } \\
\text { schedule) }\end{array} \\
\end{array}$ & $\begin{array}{l}\text { - ROM cost and schedule to mature technology is } \\
\$ 3.0 \mathrm{M} \text { over } 30 \text { months. }\end{array}$ & $\begin{array}{l}\text { - ROM cost and schedule to mature technology is } \\
\$ 4.5 \mathrm{M} \text { over } 36 \text { months. }\end{array}$ & $\begin{array}{l}\text { - ROM cost and schedule to mature technology is } \\
\$ 3.5 \mathrm{M} \text { over } 33 \text { months. }\end{array}$ \\
\hline & 3.1.3 Probability of Success & $\begin{array}{l}\text { - Probability of success for maturing technology is } \\
\text { "High". }\end{array}$ & $\begin{array}{l}\text { - Probability of success for maturing technology is } \\
\text { "Medium".(waste foaming issue was rated as a } \\
\text { High risk, even though it had marginal probability } \\
\text { of occurring; entrainment could also be an issue) }\end{array}$ & $\begin{array}{l}\text { - Probability of success for maturing technology is } \\
\text { "High". }\end{array}$ \\
\hline \multirow[t]{4}{*}{$\begin{array}{l}3.2 \text { Process Flexibility } \\
\text { and robustness }\end{array}$} & $\begin{array}{l}\text { 3.2.1 Ability to process a variety of feeds - } \\
\text { basis is counter-intuitive }\end{array}$ & $\begin{array}{l}\text { - Highly sensitive to analytes in waste feed } \\
\text { - Sensitive to a variety of physical parameters }\end{array}$ & $\begin{array}{l}\text { - Process is potentially sensitive to potassium } \\
\text { content of IPS candidate feeds }\end{array}$ & $\begin{array}{l}\text { - Selected resin has been demonstrated to meet Cs } \\
\text { removal rates for IPS candidate feeds }\end{array}$ \\
\hline & $\begin{array}{l}\text { 3.2.2 Ability to adjust process rate }- \text { turn } \\
\text { up/turn down }\end{array}$ & $\begin{array}{l}\text { - Demonstrates "High" level of flexibility to adjust } \\
\text { process rates. }\end{array}$ & $\begin{array}{l}\text { - Demonstrates "Medium" level of flexibility to } \\
\text { adjust process rates. }\end{array}$ & $\begin{array}{l}\text { - Demonstrates "Medium" level of flexibility to } \\
\text { adjust process rates. }\end{array}$ \\
\hline & 3.2.3 Flexibility to modify product & $\begin{array}{l}\text { - "High" product flexibility with } 15 \text { degrees of } \\
\text { freedom. -2stages }+\end{array}$ & $\begin{array}{l}\text { - "Medium" product flexibility with } 10 \text { degrees of } \\
\text { freedom. }\end{array}$ & $\begin{array}{l}\text { - "Medium" product flexibility with } 10 \text { degrees of } \\
\text { freedom. }\end{array}$ \\
\hline & 3.2.4 Ability to expand & $\begin{array}{l}\text { - Capacity increase would require significant } \\
\text { equipment size increases and footprint } \\
\text { modifications }\end{array}$ & $\begin{array}{l}\text { - Practical volume increases would require resizing } \\
\text { of contactors or installation of parallel lines }\end{array}$ & $\begin{array}{l}\text { - Capacity increase would require moderate size } \\
\text { increases and footprint modifications }\end{array}$ \\
\hline
\end{tabular}




\begin{tabular}{|c|c|c|c|c|}
\hline \multicolumn{5}{|c|}{ Attachment C - Cesium Separation Technology Assessment Summary Matrix } \\
\hline \multirow[t]{3}{*}{ Criteria } & Measures and Definitions & Fractional Crystallization $(\mathrm{FC})$ & Caustic-Side Solvent Extraction (CSSX) & Ion Exchange with sRF (IX-sRF) \\
\hline & 3.2.5 Recover from out of spec product & $\begin{array}{l}\text { - System would require minor tank storage changes } \\
\text { to handle out-of-spec product while recycling }\end{array}$ & $\begin{array}{l}\text { - Installation of recycle piping would be required at } \\
\text { an ROM cost of }<\$ 5 \mathrm{M}\end{array}$ & $\begin{array}{l}\text { - System would require minor tank storage changes } \\
\text { to handle out-of-spec product while recycling }\end{array}$ \\
\hline & $\begin{array}{l}\text { 3.2.6 Technology applicability to other } \\
\text { DOE complex projects - revisit in } \\
\text { programmatic aspects evaluation }\end{array}$ & - "No" applicability to other DOE complex projects. & $\begin{array}{l}\text { - "High" applicability to other DOE complex } \\
\text { projects. }\end{array}$ & $\begin{array}{l}\text { - "High" applicability to other DOE complex } \\
\text { projects. }\end{array}$ \\
\hline \multirow{10}{*}{$\begin{array}{l}4 \text { OPERABILITY AND } \\
\text { MAINTAINABILITY } \\
4.1 \quad \text { Ease of Process } \\
\text { control and operation }\end{array}$} & $\begin{array}{l}\text { 4.1.1 Minimize number and frequency of } \\
\text { surveillances }\end{array}$ & $\begin{array}{l}\text { - } 58 \text { process parameters and } 11 \text { sump leak detectors } \\
\text { requiring routine measurements and recordings. }\end{array}$ & $\begin{array}{l}\text { - } 38 \text { process parameters, } 20 \text { sump leak detectors and } \\
98 \text { other equipment related data points requiring } \\
\text { routine measurements and recordinos }\end{array}$ & $\begin{array}{l}\text { - } 26 \text { process parameters and } 18 \text { sump leak detectors } \\
\text { requiring routine measurements and recordings. }\end{array}$ \\
\hline & $\begin{array}{l}\text { 4.1.2 Minimize number of people to } \\
\text { operate }\end{array}$ & - Estimated 10 people to operate. & - Estimated 11 people to operate. & - Estimated 10 people to operate. \\
\hline & 4.1.3 Ease of startup and shutdown & $\begin{array}{l}\text { - Startup and shut down has medium complexity } \\
\text { among these three systems. }\end{array}$ & $\begin{array}{l}\text { - Startup and shut down is the most time consuming } \\
\text { and complex among these three systems. }\end{array}$ & $\begin{array}{l}\text { - Startup and shut down is relatively simple with the } \\
\text { lowest complexity among these three systems. }\end{array}$ \\
\hline & 4.1.4 Minimize system complexity & $\begin{array}{l}\text { - System complexity is moderate due to basic } \\
\text { evaporation equipment with specialized control } \\
\text { functions. }\end{array}$ & $\begin{array}{l}\text { - System complexity is very high with } 85 \text { active } \\
\text { components with associated instrumentation. } \\
\text { System and process flowsheets are highly complex } \\
\text { with potential for more process upsets. }\end{array}$ & $\begin{array}{l}\text { - System complexity is considered to be low based } \\
\text { on passive nature of resin columns. }\end{array}$ \\
\hline & $\begin{array}{l}\text { 4.1.5 Minimize number of chemicals } \\
\text { needed }\end{array}$ & - No chemicals used in the basic system operation. & $\begin{array}{l}\text { - In addition to } \mathrm{NaOH}, \mathrm{NaNO} \text {, and } \mathrm{HNO} \text {, four } \\
\text { other organic chemicals are used in the process. }\end{array}$ & $\begin{array}{l}\text { - Three chemicals, } \mathrm{NaOH}, \mathrm{NaNO} 2 \text {, and } \mathrm{HNO} 3 \text { are } \\
\text { used in the process. }\end{array}$ \\
\hline & $\begin{array}{l}\text { 4.1.6 Minimize number of process and } \\
\text { regulatory samples }\end{array}$ & $\begin{array}{l}\text { - Process sampling needs include feed and product } \\
\text { streams and steam condensate. Batch sampling of } \\
\text { liquid effluent stream going to ETF will be } \\
\text { required. }\end{array}$ & $\begin{array}{l}\text { - Process sampling needs is anticipated for feed and } \\
\text { product streams. In addition, aqueous and organic } \\
\text { streams from contactors, process chemicals will be } \\
\text { required on a regular basis. Spent solvent will be } \\
\text { sampled on a batch basis to meet regulatory } \\
\text { disposal requirements. }\end{array}$ & $\begin{array}{l}\text { - Process sampling needs is anticipated for feed and } \\
\text { product streams. Aqueous make ups should be } \\
\text { sampled on a regular basis. Spent resin sampling } \\
\text { will be required on a batch basis to assure disposal } \\
\text { requirement conformance. }\end{array}$ \\
\hline & 4.1.7 Batch verses continuous & - Operates on a semi-continuous or campaign basis. & - Operates on a continuous basis. & - Operated only on a batch basis. \\
\hline & 4.1.8 Ease of entry and exit from standby & - Minor impact for standby restart activities. & $\begin{array}{l}\text { - Significant operational impacts for any shutdown } \\
\text { or standby condition to restart activities. }\end{array}$ & $\begin{array}{l}\text { - Minimal impacts for a short time standby restart } \\
\text { activities. }\end{array}$ \\
\hline & 4.1.9 Wide operating margin & $\begin{array}{l}\text { - Can be applied to wide range of feed containing } \\
\text { various chemicals. } \\
\text { - Relatively tight range of temperature and pressure } \\
\text { required for crystal formation }\end{array}$ & $\begin{array}{l}\text { - Does not have a wide operating range for most } \\
\text { flow sheet parameters with the exception of the } \\
\text { washing and scrubbing functions. } \\
\text { - Aqueous/organic ratio is critical and needs to be } \\
\text { tightly controlled }\end{array}$ & $\begin{array}{l}\text { - Narrow range of application, based on } \mathrm{Na} \\
\text { concentration } \\
\text { - Relatively forgiving process based on temperature } \\
\text { range. }\end{array}$ \\
\hline & $\begin{array}{l}\text { 4.1.10 Complexity of transfers to, from and } \\
\text { within Tank Farms }\end{array}$ & $\begin{array}{l}\text { - Effluent stream transfer to ETF for final disposal. } \\
\text { - Return stream to DSTs }\end{array}$ & $\begin{array}{l}\text { - Standard tank farm transfers (requires no special } \\
\text { equipment or processes to transfers). } \\
\text { - Return stream to DSTs }\end{array}$ & $\begin{array}{l}\text { - No special or additional tank waste transfer } \\
\text { anticipated. } \\
\text { - Return stream to DSTs }\end{array}$ \\
\hline
\end{tabular}




\begin{tabular}{|c|c|c|c|c|}
\hline \multicolumn{5}{|c|}{ Attachment C - Cesium Separation Technology Assessment Summary Matrix } \\
\hline Criteria & Measures and Definitions & \multirow[b]{2}{*}{$\begin{array}{l}\text { Fractional Crystallization (FC) } \\
\text { - Estimated process related components requiring } \\
\text { hands-on or contact maintenance are: One waste } \\
\text { feed pump, } 2 \text { centrifuges, } 15 \text { process pumps, } \\
\text { undetermined numbers of flow meters, and flow } \\
\text { control valves. } \\
\text { - Has medium number of components that may } \\
\text { require contact maintenance. }\end{array}$} & \multirow[b]{2}{*}{$\begin{array}{l}\text { Caustic-Side Solvent Extraction (CSSX) } \\
\text { - Estimated process related components requiring } \\
\text { hands-on or contact maintenance are: One waste } \\
\text { feed pump, } 41 \text { contactors; } 7 \text { primary process } \\
\text { pumps, undetermined numbers of flow meters, and } \\
\text { flow control valves; seven transfer pumps } \\
\text { associated with aqueous chemical make-up area. } \\
\text { - Has highest number of components that may } \\
\text { require contact maintenance. }\end{array}$} & \multirow[b]{2}{*}{$\begin{array}{l}\text { Ion Exchange with sRF (IX-sRF) } \\
\text { - Estimated process related components requiring } \\
\text { hands-on or contact maintenance are: One waste } \\
\text { feed pump, } 2 \text { ion exchange columns; } 13 \text { process } \\
\text { pumps, undetermined numbers of flow meters, and } \\
\text { flow control valves. } \\
\text { - Has lowest number of components that may } \\
\text { require contact maintenance. }\end{array}$} \\
\hline ALARA & $\begin{array}{l}4.2 .1 \text { Less required contact maintenance is } \\
\text { better, etc. }\end{array}$ & & & \\
\hline \multirow[t]{2}{*}{ 4.3 Reliability } & 4.3.1 Number of active components & $\begin{array}{l}\text { - System has } 21 \text { active components consisting of } \\
\text { pumps, centrifuges, chilled water skids, and } \\
\text { building ventilation system. }\end{array}$ & $\begin{array}{l}\text { - System has } 60 \text { active components consisting of } \\
\text { pumps and centrifugal contactors, and building } \\
\text { ventilation system. }\end{array}$ & $\begin{array}{l}\text { - System has } 14 \text { active components consisting of } \\
\text { pumps and building ventilation system. }\end{array}$ \\
\hline & 4.3.2 Reliability of analogous systems & $\begin{array}{l}\text { - 242-A Evaporators provides strong analogous } \\
\text { reliability data support. }\end{array}$ & $\begin{array}{l}\text { - The use of centrifugal contactors has been } \\
\text { successful at SRS. }\end{array}$ & $\begin{array}{l}\text { - Ion exchange operation does provide some } \\
\text { improved reliability, but sRF resin reliability data } \\
\text { is not available. } \\
\text { - Large-scale IX has been operated successfully at } \\
\text { Hanford for many years, e.g., B-Plant separations } \\
\text { of Cs and Sr. }\end{array}$ \\
\hline \multirow[t]{5}{*}{$\begin{array}{l}4.4 \quad \text { Ease and } \\
\text { frequency of maintenance }\end{array}$} & 4.4.1 Minimize number of support systems & $\begin{array}{l}\text { - Requires } 3 \text { standard services of air, water, and } \\
\text { cooling water. } \\
\text { - Will also require steam to be provided } \\
\text { - Requires } 1 \text { chemical support service. } \\
\end{array}$ & $\begin{array}{l}\text { - Requires } 3 \text { standard services of air, water and } \\
\text { cooling water. } \\
\text { - Requires six chemical support services. }\end{array}$ & $\begin{array}{l}\text { - Requires } 3 \text { standard services of air, water and } \\
\text { cooling water. } \\
\text { - Requires five chemical support services. }\end{array}$ \\
\hline & $\begin{array}{l}\text { 4.4.2 } \text { Minimize number and frequency of } \\
\text { PM's }\end{array}$ & $\begin{array}{l}\text { - Moderate level of PMs required due to the large } \\
\text { number of rotating components, and necessary } \\
\text { piping and control system. }\end{array}$ & $\begin{array}{l}\text { - Extensive level of PMs required due to the large } \\
\text { number of rotating components, and necessary } \\
\text { piping and control system. }\end{array}$ & $\begin{array}{l}\text { - Minimal level of PMs required due to the passive } \\
\text { operation of a majority of the technology, and low } \\
\text { number of process components required. }\end{array}$ \\
\hline & 4.4.3 Minimize maintenance in-zone entries & $\begin{array}{l}\text { - Moderate amount of equipment requiring } \\
\text { maintenance in-zone. }\end{array}$ & $\begin{array}{l}\text { - Extensive amount of equipment requiring } \\
\text { maintenance in-zone. }\end{array}$ & $\begin{array}{l}\text { - Moderate amount of equipment requiring } \\
\text { maintenance in-zone. } \\
\text { - Fewer in-zone maintenance items; most of } \\
\text { equipment, e.g., pumps, are located in cold } \\
\text { chemical area }\end{array}$ \\
\hline & $\begin{array}{ll}\text { 4.4.4 } & \text { Minimize specialized equipment and } \\
\text { parts } & \\
\end{array}$ & - Uses commercially available components & $\begin{array}{l}\text { - Uses specialized components which may limit the } \\
\text { availability of components and parts. }\end{array}$ & $\begin{array}{l}\text { - Uses commercially available components } \\
\text { - Assumes external jacketed cooling to IX column }\end{array}$ \\
\hline & 4.4.5 Minimize tank entries - N/A & - No tank entries are required for this technology. & - No tank entries are required for this technology. & - No tank entries are required for this technology. \\
\hline \multirow[t]{3}{*}{$\begin{array}{l}4.5 \quad \text { Ease of } \\
\text { Implementation }\end{array}$} & 4.5.1 Ease of training & $\begin{array}{l}\text { - Above average amount of training is anticipated } \\
\text { due to large number of components and complex } \\
\text { process control. }\end{array}$ & $\begin{array}{l}\text { - Extensive amount of training is anticipated due to } \\
\text { large number of components and complex process } \\
\text { control. }\end{array}$ & $\begin{array}{l}\text { - Nominal amount of training is anticipated due to } \\
\text { small number of components and simpler process } \\
\text { control. }\end{array}$ \\
\hline & 4.5.2 Complexity of procedures & - Above average procedure complexity. & - Extensive complexity in procedures. & - Nominal complex procedures. \\
\hline & $\begin{array}{l}\text { 4.5.3 Similar to other process facilities on } \\
\text { site }\end{array}$ & $\begin{array}{l}\text { - Technology is similar to evaporator system with } \\
\text { familiarity at Hanford. }\end{array}$ & $\begin{array}{l}\text { - Technology is not used at Hanford but is being } \\
\text { implemented at SRS. }\end{array}$ & $\begin{array}{l}\text { - Technology is similar to other ion exchange } \\
\text { systems used at Hanford and will be implemented } \\
\text { at pretreatment facility of the WTP project. }\end{array}$ \\
\hline $\begin{array}{l}4.6 \quad \text { Liquid/solid } \\
\text { secondary waste }\end{array}$ & $\begin{array}{l}\text { 4.6.1 Waste handling compatible with } \\
\text { existing systems as defined by DOE Order } \\
420.1 \mathrm{~B}\end{array}$ & $\begin{array}{l}\text { - Can be designed to be compliant with DOE-Order } \\
420.1 \mathrm{~B} \text {, } \\
\text { - Not a significant issue }\end{array}$ & $\begin{array}{l}\text { - Can be designed to be compliant with DOE-Order } \\
420.1 \mathrm{~B} \text {. } \\
\text { - Not a significant issue }\end{array}$ & $\begin{array}{l}\text { - Can be designed to be compliant with DOE-Order } \\
420.1 \mathrm{~B} \text {. } \\
\text { - Not a significant issue }\end{array}$ \\
\hline
\end{tabular}




\begin{tabular}{|c|c|c|c|c|}
\hline \multicolumn{5}{|c|}{ Attachment C-Cesium Separation Technology Assessment Summary Matrix } \\
\hline \multirow[t]{2}{*}{ Criteria } & Measures and Definitions & Fractional Crystallization $(\mathrm{FC})$ & Caustic-Side Solvent Extraction (CSSX) & Ion Exchange with sRF (IX-sRF) \\
\hline & $\begin{array}{l}\text { 4.6.2 Minimize operational impacts } \\
\text { associated with hazardous (generated) waste } \\
\text { handling }\end{array}$ & $\begin{array}{l}\text { - Suited for continuous operation. } \\
\text { - Secondary waste is routed to ETF with no } \\
\text { abnormal operational impact. }\end{array}$ & $\begin{array}{l}\text { - Suited for continuous operation. } \\
\text { - Cs product requiring volume reduction is routed to } \\
\text { evaporator with no abnormal operational impact. } \\
\text { - Disposal of spent organics }\end{array}$ & $\begin{array}{l}\text { - Suited for continuous operation with planned } \\
\text { shutdown for resin change outs. } \\
\text { - Used resin requires special handling and disposal } \\
\text { as LLW. This can be designed in the facility to } \\
\text { minimize operational waste handling impacts. }\end{array}$ \\
\hline \multirow[t]{3}{*}{$\begin{array}{l}5 \text { PROGRAMMATIC } \\
\text { ASPECTS } \\
5.1 \quad \text { Cost Impact }\end{array}$} & $\begin{array}{l}\text { 5.1.1 Capital costs (for comparative } \\
\text { purposes only) }\end{array}$ & $\begin{array}{l}\text { - With expected accuracy range of }-30 \% \text { to }+50 \% \text { : } \\
\text { FC - CFF capital costs }=\$ 64 \mathrm{M} \text { to } \$ 140 \mathrm{M} \text {, } \\
\text { FC - RMF capital costs }=\$ 75 \mathrm{M} \text { to } \$ 160 \mathrm{M} \text {. }\end{array}$ & $\begin{array}{l}\text { - With expected accuracy range of }-30 \% \text { to }+50 \% \text { : } \\
\text { CSSX - CFF capital costs }=\$ 82 \mathrm{M} \text { to } \$ 180 \mathrm{M} \text {, } \\
\text { CSSX- RMF capital costs }=\$ 87 \mathrm{M} \text { to } \$ 190 \mathrm{M} \text {. }\end{array}$ & $\begin{array}{l}\text { - With expected accuracy range of }-30 \% \text { to }+50 \% \text { : } \\
\text { IX }- \text { CFF capital costs }=\$ 54 \mathrm{M} \text { to } \$ 120 \mathrm{M} \text {, } \\
\text { IX - RMF capital costs }=\$ 59 \mathrm{M} \text { to } \$ 130 \mathrm{M} \text {. }\end{array}$ \\
\hline & $\begin{array}{l}\text { 5.1.2 Life cycle costs (for comparative } \\
\text { purposes only) }\end{array}$ & $\begin{array}{l}\text { - With expected accuracy range of }-30 \% \text { to }+50 \% \\
\text { (applied to capital cost contribution): } \\
\text { FC - CFF life cycle costs (w/o T\&Rs) = \$180M to } \\
\$ 250 \mathrm{M}, \mathrm{FC}-\mathrm{RMF} \text { life cycle costs (w/o T\&Rs) = } \\
\$ 210 \mathrm{M} \text { to } \$ 290 \mathrm{M} \text {. } \\
\text { - } 2 \text { SST retrievals add } \$ 50 \mathrm{M} \text { of non-project cost in } \\
\text { 4th year of mission; these are accelerated (not } \\
\text { new) costs }\end{array}$ & $\begin{array}{l}\text { - With expected accuracy range of }-30 \% \text { to }+50 \% \\
\text { (applied to capital cost contribution): } \\
\text { CSSX - CFF life cycle costs }(w / \text { evap) }=\$ 190 \mathrm{M} \text { to } \\
\$ 290 \mathrm{M} ; \text { CSSX - RMF life cycle costs (w/ evap) }= \\
\$ 210 \mathrm{M} \text { to } \$ 310 \mathrm{M} \text {. }\end{array}$ & $\begin{array}{l}\text { - With expected accuracy range of }-30 \% \text { to }+50 \% \\
\text { (applied to capital cost contribution): } \\
\text { IX - CFF life cycle costs }=\$ 140 \mathrm{M} \text { to } \$ 200 \mathrm{M} \text {, } \\
\text { IX - RMF life cycle costs }=\$ 150 \mathrm{M} \text { to } \$ 220 \mathrm{M} \text {. }\end{array}$ \\
\hline & $\begin{array}{l}\text { 5.1.3 Cost profile (for comparative } \\
\text { purposes only) }\end{array}$ & $\begin{array}{l}\text { - When non-project cost for SST retrievals are } \\
\text { included, FC life-cycle cost is the highest (for } \\
\text { comparative purposes only) }\end{array}$ & $\begin{array}{l}\text { - Both the highest capital cost and highest life cycle } \\
\text { cost result from pairing CSSX with RMF. (for } \\
\text { comparative purposes only) }\end{array}$ & $\begin{array}{l}\text { - Both the lowest capital cost and life cycle cost } \\
\text { result from pairing IX-sRF with CFF. (for } \\
\text { comparative purposes only) }\end{array}$ \\
\hline \multirow[t]{4}{*}{ 5.2 $\quad$ Schedule Impact } & $\begin{array}{l}\text { 5.2.1 Overall schedule (confidence) - for } \\
\text { comparative purposes only }\end{array}$ & $\begin{array}{l}\text { - At } 50 \% \text { of probability of on-time completion the } \\
\text { estimated schedule duration is } 100 \text { months, from } \\
\text { CD-1 to Startup completion. }\end{array}$ & $\begin{array}{l}\text { - At } 50 \% \text { of probability of on-time completion the } \\
\text { estimated schedule duration is } 110 \text { months, from } \\
\text { CD-1 to Startup completion. }\end{array}$ & $\begin{array}{l}\text { - At } 50 \% \text { of probability of on-time completion the } \\
\text { estimated schedule duration is } 90 \text { months, from } \\
\text { CD-1 to Startup completion. }\end{array}$ \\
\hline & 5.2.2 Licensing & $\begin{array}{l}\text { - Due to existing knowledge and experience } \\
\text { licensing activities should not adversely impact. }\end{array}$ & $\begin{array}{l}\text { - Due to lack of Hanford specific performance } \\
\text { knowledge, CSSX will require additional efforts } \\
\text { and time to support licensing activities. }\end{array}$ & $\begin{array}{l}\text { - Due to existing knowledge and experience } \\
\text { licensing activities should not adversely impact. }\end{array}$ \\
\hline & $\begin{array}{ll}5.2 .3 & \text { Permitting }\end{array}$ & $\begin{array}{l}\text { - Assuming timely completion of TC\&WM EIS and } \\
32 \text { months permitting process per Tri-Party } \\
\text { Agreement, RCRA part B permitting for FC will } \\
\text { not impact start of Construction. }\end{array}$ & $\begin{array}{l}\text { - Assuming timely completion of TC\&WM EIS and } \\
32 \text { months permitting process per Tri-Party } \\
\text { Agreement, RCRA part B permitting for CSSX } \\
\text { will not impact start of Construction. }\end{array}$ & $\begin{array}{l}\text { - Assuming timely completion of TC\&WM EIS and } \\
32 \text { months permitting process per Tri-Party } \\
\text { Agreement, RCRA part B permitting for IX-sRF } \\
\text { will not impact start of Construction. }\end{array}$ \\
\hline & 5.2.4 D\&D & $\begin{array}{l}\text { - Considerations for D\&D will be accommodated } \\
\text { during the IPS design. Negligible impact on IPS } \\
\text { schedule. }\end{array}$ & $\begin{array}{l}\text { - Considerations for D\&D will be accommodated } \\
\text { during the IPS design. Negligible impact on IPS } \\
\text { schedule. }\end{array}$ & $\begin{array}{l}\text { - Considerations for D\&D will be accommodated } \\
\text { during the IPS design. Negligible impact on IPS } \\
\text { schedule. }\end{array}$ \\
\hline DST Space & $\begin{array}{l}\text { 5.3.1 How fast DST space is made } \\
\text { available }\end{array}$ & $\begin{array}{l}\text { - Recovers DST space at a rate of } 1.3 \mathrm{M} \mathrm{gal} / \mathrm{yr} \text { to } \\
1.5 \mathrm{M} \text { gal yr dependent upon what extent the } \\
\text { potassium limit of } 0.1 \mathrm{M} \text { is imposed, during first } 3 \\
\text { years. } \\
\text { - Recovers DST space at } 0.6 \mathrm{M} \mathrm{gal} \mathrm{yr} \mathrm{to} 2.2 \mathrm{M} \mathrm{gal} / \mathrm{yr} \\
\text { dependent upon what extent the potassium limit of } \\
0.1 \mathrm{M} \text { is imposed, during the } 4 \text { th year. } \\
\text { - N/A for } 5 \text { th year since recovery is from SSTs (i.e. } \\
\text { DST space is instead consumed) }\end{array}$ & $\begin{array}{l}\text { - Recovers DST space at a rate of } 520 \mathrm{~K} \mathrm{gal} / \mathrm{yr} \text {. } \\
\text { - } 205 \mathrm{~K} \text { gal } / \mathrm{yr} \text { of DST space can be recovered if the } \\
\text { current operating limit of } 0.8 \mathrm{Ci} / \text { liter for the } 242-\mathrm{A} \\
\text { Evaporator is imposed. } \\
\text { - If the higher DSA limit of } 1 / 5 \mathrm{Ci} / \text { liter for the } \\
\text { evaporator is used an additional } 365 \mathrm{kgal} / \mathrm{yr} \text { would } \\
\text { be achieved. }\end{array}$ & $\begin{array}{l}\text { - Recovers DST space at a rate of } 725 \mathrm{~K} \mathrm{gal} / \mathrm{yr} \text {. } \\
\text { - A small amount of DST space }(15 \mathrm{k} / \mathrm{yr}) \text { can be } \\
\text { recovered if the current operating limit of } 0.8 \\
\text { Ci/liter for the } 242 \text {-A Evaporator is imposed. }\end{array}$ \\
\hline
\end{tabular}




\begin{tabular}{|c|c|c|c|c|}
\hline \multicolumn{5}{|c|}{ Attachment C - Cesium Separation Technology Assessment Summary Matrix } \\
\hline \multirow[t]{2}{*}{ Criteria } & Measures and Definitions & Fractional Crystallization $(\mathrm{FC})$ & Caustic-Side Solvent Extraction (CSSX) & Ion Exchange with sRF (IX-sRF) \\
\hline & 5.3.2 Amount of DST space & $\begin{array}{l}-2.8 \mathrm{M}-6.4 \mathrm{M} \text { gallons dependent upon what extent } \\
\text { the potassium limit of } 0.1 \mathrm{M} \text { is imposed. }\end{array}$ & $\begin{array}{l}\text { - Recovers } 3.6 \mathrm{M} \text { gallons of DST space. (assumes } \\
\text { the WTP solubility limit for Al is imposed and the } \\
\text { waste volume of the Cs-loaded stream is further } \\
\text { reduced at the } 242-\mathrm{A} \text { evaporator) }\end{array}$ & $\begin{array}{l}\text { - Recovers } 3.6 \mathrm{M} \text { gallons of DST space. (assumes } \\
\text { the WTP solubility limit for Al is imposed and the } \\
\text { waste volume of the Cs-loaded stream is further } \\
\text { reduced at the } 242-\mathrm{A} \text { evaporator) }\end{array}$ \\
\hline \multirow[t]{8}{*}{$\begin{array}{l}5.4 \quad \text { Impacts to WTP } \\
\text { and Supplemental } \\
\text { Treatment, positive and } \\
\text { negative }\end{array}$} & 5.4.1 Production rate impact & $\begin{array}{l}\text { - Will provide required feed rate of } 0.192 \mathrm{MT} \mathrm{Na} / \mathrm{hr} \\
\text { to meet WTP/supplemental treatment production } \\
\text { requirements. } \\
\text { - Sulfate concentration in FC feed may adversely } \\
\text { impact the production rate. } \\
\text { - Potentially will have to add Na to maintain proper } \\
\text { feed solubility to WTP Pretreatment (based upon } \\
\text { WTP Al solubility curves). }\end{array}$ & $\begin{array}{l}\text { - Will provide required feed rate of } 0.192 \mathrm{MT} \mathrm{Na} / \mathrm{hr} \\
\text { to meet WTP/supplemental treatment production } \\
\text { requirements. } \\
\text { - Assessments of impacts of chemical composition } \\
\text { of the feed needs to be made. } \\
\text { - Approximately } 1 / 3 \text { of } \mathrm{Na} \text { is cold chemical addition } \\
\text { to maintain Al solubility } \\
\text { - Has additional Na load to maintain proper feed } \\
\text { solubility (based upon WTP Al solubility curves). }\end{array}$ & $\begin{array}{l}\text { - Will provide required feed rate of } 0.192 \mathrm{MT} \mathrm{Na} / \mathrm{hr} \\
\text { to meet WTP/supplemental treatment production } \\
\text { requirements. } \\
\text { - Assessments of impacts of chemical composition } \\
\text { of the feed needs to be made } \\
\text { - Has a NaOH load same as WTP ion exchange } \\
\text { system. } \\
\text { - Approximately } 1 / 3 \text { of Na is cold chemical addition } \\
\text { to maintain Al solubility } \\
\text { - Has additional Na load to maintain proper feed } \\
\text { solubility (based upon WTP Al solubility curves). }\end{array}$ \\
\hline & 5.4.2 Mission duration & - No impact & - No impact & - No impact \\
\hline & $\begin{array}{l}\text { 5.4.3 Number of high and low level } \\
\text { packages (need to revise for 5-year mission) }\end{array}$ & $\begin{array}{l}\text { - Produces 4,200 ILAW canisters based on Na } \\
\text { inventory of the LAW feed. } \\
\text { - Additional canisters (LAW and/or HLW) will be } \\
\text { required to be made depending on the disposition } \\
\text { of Na that is the part of high-Cs product stream. }\end{array}$ & $\begin{array}{l}\text { - Produces } 12,300 \mathrm{ILAW} \text { canisters based on Na } \\
\text { inventory of the LAW feed. }\end{array}$ & $\begin{array}{l}\text { - Produces } 12,200 \text { ILAW canisters based on Na } \\
\text { inventory of the LAW feed. }\end{array}$ \\
\hline & $\begin{array}{l}\text { 5.4.4 Lessons Learned benefits for WTP } \\
\text { pretreatment }\end{array}$ & $\begin{array}{l}\text { - There will not be "lessons learned" from the } \\
\text { operations and maintenance of FC equipment in } \\
\text { IPS. }\end{array}$ & $\begin{array}{l}\text { - There will not be "lessons learned" from the } \\
\text { operations and maintenance of CSSX equipment in } \\
\text { IPS. }\end{array}$ & $\begin{array}{l}\text { - Provides lessons learned benefits to WTP ion } \\
\text { exchange process development, operation and } \\
\text { maintenance activities. }\end{array}$ \\
\hline & $\begin{array}{l}\text { 5.4.5 Technology transfer to WTP and/or } \\
\text { Supplemental Treatment }\end{array}$ & - Not applicable & - Not applicable & - Not applicable. \\
\hline & 5.4.6 ALARA & $\begin{array}{l}\text { - Potential ALARA impact to WTP LAW facility, } \\
\text { with current design because of higher DF. }\end{array}$ & - No ALARA impact to WTP LAW facility. & - No ALARA impact to WTP LAW facility. \\
\hline & 5.4.7 Diversity of technology & $\begin{array}{l}\text { - Provides diversity of technology for use at } \\
\text { Hanford. }\end{array}$ & $\begin{array}{l}\text { - Provides diversity of technology for use at } \\
\text { Hanford. }\end{array}$ & - No new technology for use at Hanford. \\
\hline & $\begin{array}{l}\text { 5.4.8 Positive programmatic impacts and } \\
\text { opportunities }\end{array}$ & $\begin{array}{l}\text { - Provides alternative evaporator capability } \\
\text { - Provides possibility of using grout for } \\
\text { immobilizing TC-free LAW }\end{array}$ & $\begin{array}{l}\text { - No programmatic benefits have been identified } \\
\text { yet. }\end{array}$ & $\begin{array}{l}\text { - Provides potential cost reduction benefits by } \\
\text { combining IPS and WTP sRF technology } \\
\text { deployment activities. } \\
\text { - Potential use of IX for Tc removal } \\
\end{array}$ \\
\hline \multirow[t]{3}{*}{$\begin{array}{l}5.5 \quad \text { Impacts to other } \\
\text { facilities e.g., ETF, LAB, } \\
\text { IDF (see regulatory } \\
\text { assessment for IDF } \\
\text { impacts) }\end{array}$} & $\begin{array}{l}\text { 5.5.1 Analytical equipment, methods, and } \\
\text { capacity }\end{array}$ & $\begin{array}{l}\text { - WTP LAW feed stream (cesium-depleted product) } \\
\text { will be analyzed at WTP lab; Cesium-rich product } \\
\text { analyzed at 222-S lab; process control analyses } \\
\text { performed on-line at IPS. }\end{array}$ & $\begin{array}{l}\text { WTP LAW feed stream (cesium-depleted product) } \\
\text { will be analyzed at WTP lab; Cesium-rich product } \\
\text { analyzed at 222-S lab; process control analyses } \\
\text { performed on-line at IPS. }\end{array}$ & $\begin{array}{l}\text { - WTP LAW feed stream (cesium-depleted product) } \\
\text { will be analyzed at WTP 1ab; Cesium-rich product } \\
\text { analyzed at 222-S lab; process control analyses } \\
\text { performed on-line at IPS. }\end{array}$ \\
\hline & & $\begin{array}{l}\text { - Amount of lag storage for product batches may } \\
\text { require adjustment based on laboratory analysis } \\
\text { turn-around time. }\end{array}$ & $\begin{array}{l}\text { - Amount of lag storage for product batches may } \\
\text { require adjustment based on laboratory analysis } \\
\text { turn-around time. }\end{array}$ & $\begin{array}{l}\text { - Amount of lag storage for product batches may } \\
\text { require adjustment based on laboratory analysis } \\
\text { turn-around time. }\end{array}$ \\
\hline & 5.5.2 Compliance to ETF WAC & - Process condensates from FC meet ETF's WAC. & - No impact to ETF. & - No impact to ETF. \\
\hline
\end{tabular}




\begin{tabular}{|c|c|c|c|c|}
\hline \multicolumn{5}{|c|}{ Attachment C - Cesium Separation Technology Assessment Summary Matrix } \\
\hline Criteria & Measures and Definitions & Fractional Crystallization $(\mathrm{FC})$ & Caustic-Side Solvent Extraction (CSSX) & Ion Exchange with sRF (IX-sRF) \\
\hline & 5.5.3 ALARA & $\begin{array}{l}\text { - Because FC is being included as part of the new } \\
\text { IPS facility, ALARA will be incorporated into its } \\
\text { process design. }\end{array}$ & $\begin{array}{l}\text { - Because CSSX is being included as part of the new } \\
\text { IPS facility, ALARA will be incorporated into its } \\
\text { process design. }\end{array}$ & $\begin{array}{l}\text { - Because IX-sRF is being included as part of the } \\
\text { new IPS facility, ALARA will be incorporated into } \\
\text { its process design. }\end{array}$ \\
\hline & 5.5.4 Number of Evaporator campaigns & $\begin{array}{l}\text { - No evaporator campaigns required because } \\
\text { cesium-enriched stream meets DST density } \\
\text { specifications. }\end{array}$ & $\begin{array}{l}\text { - Using the } 0.8 \mathrm{Ci} / \text { /iter operating limit for } 242-\mathrm{A} \\
\text { evaporator, } 5 \text { evaporator campaigns will provide } \\
\text { an additional } 1.0 \mathrm{M} \text { gal of DST space }\end{array}$ & $\begin{array}{l}\text { - No evaporator campaigns are required because } \\
\text { cesium-enriched stream is near or exceeds the } 0.8 \\
\mathrm{Ci} / \text { liter operating limit for } 242-\mathrm{A} \text { Evaporator. }\end{array}$ \\
\hline \multirow[t]{2}{*}{$\begin{array}{l}5.6 \quad \text { Resources and } \\
\text { materials }\end{array}$} & $\begin{array}{l}\text { 5.6.1 Availability of Key Skills, Critical } \\
\text { Materials, Qualified Vendors }\end{array}$ & $\begin{array}{l}\text { - Does not use any specialty chemicals or material. } \\
\text { - Uses engineered equipment to be designed and } \\
\text { fabricated by experienced vendors. }\end{array}$ & $\begin{array}{l}\text { - Costner Industries Nevada Corp. is the } \\
\text { experienced commercial company who is } \\
\text { experienced with supplying centrifugal } \\
\text { contractors, due to their working partnership with } \\
\text { SRNL. Other basic commercial suppliers do exist. } \\
\text { - Solvents used are proprietary: such as BOB } \\
\text { CalixC6, TOA and Cs-7SB. Suppliers are single } \\
\text { source and may have to pay premium price to } \\
\text { obtain these chemicals. - verify that these are } \\
\text { proprietary (check with Parsons) }\end{array}$ & $\begin{array}{l}\text { - Two resin manufacturing facilities have been } \\
\text { identified and prepared product tested. } \\
\text { - Long term supply strategy for resin must be } \\
\text { developed. }\end{array}$ \\
\hline & 5.6.2 Stability of Critical Resource Pricing & - Qualified fabricators may be difficult to find & - Qualified fabricators may be difficult to find & - Qualified fabricators may be difficult to find \\
\hline
\end{tabular}


RPP-RPT-37740, Rev 0

ATTACHMENT D

IPS SCHEDULES 
Caustic Side Solvent Extraction

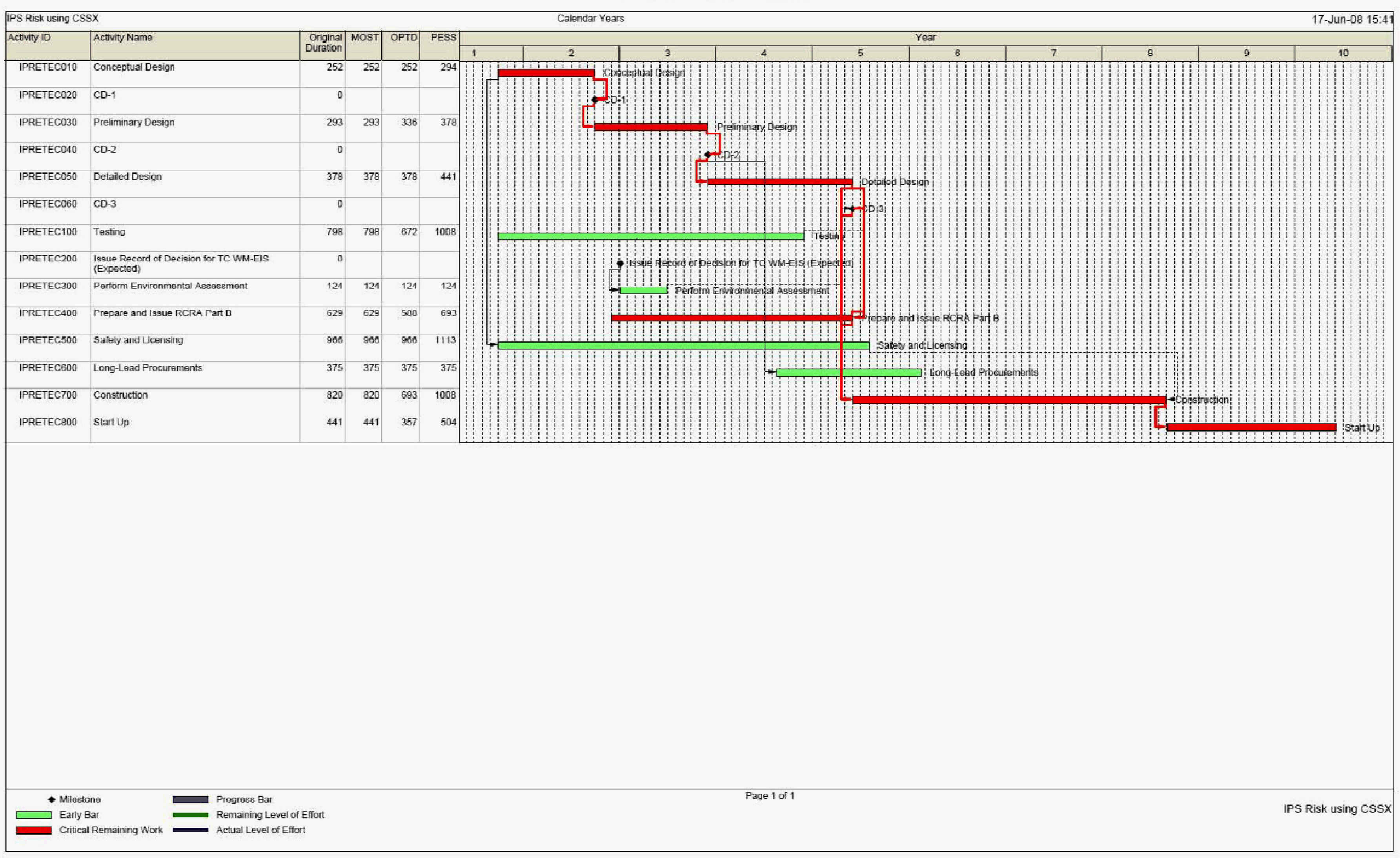




\section{Fractional Crystallization}

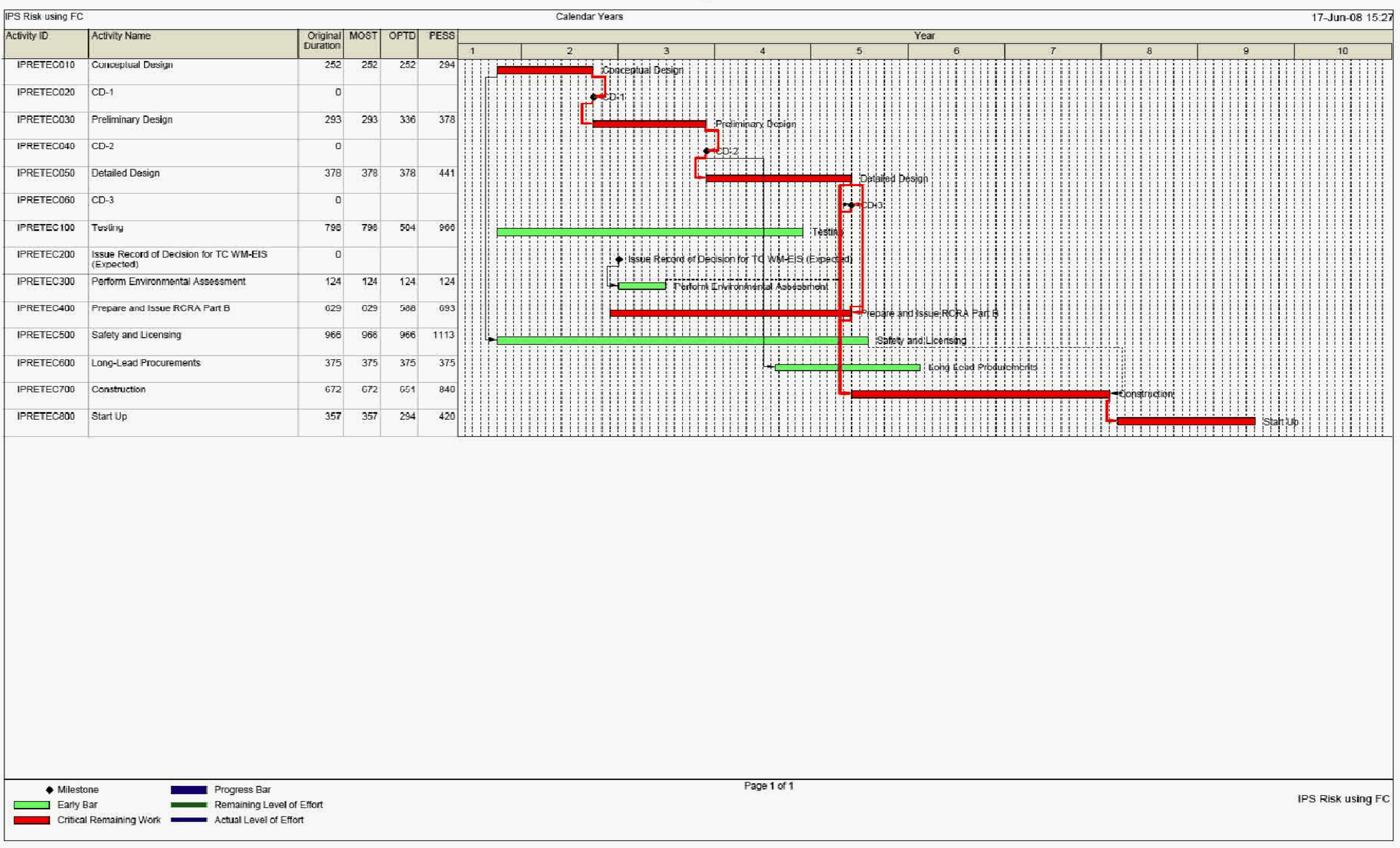


Ion Exchange

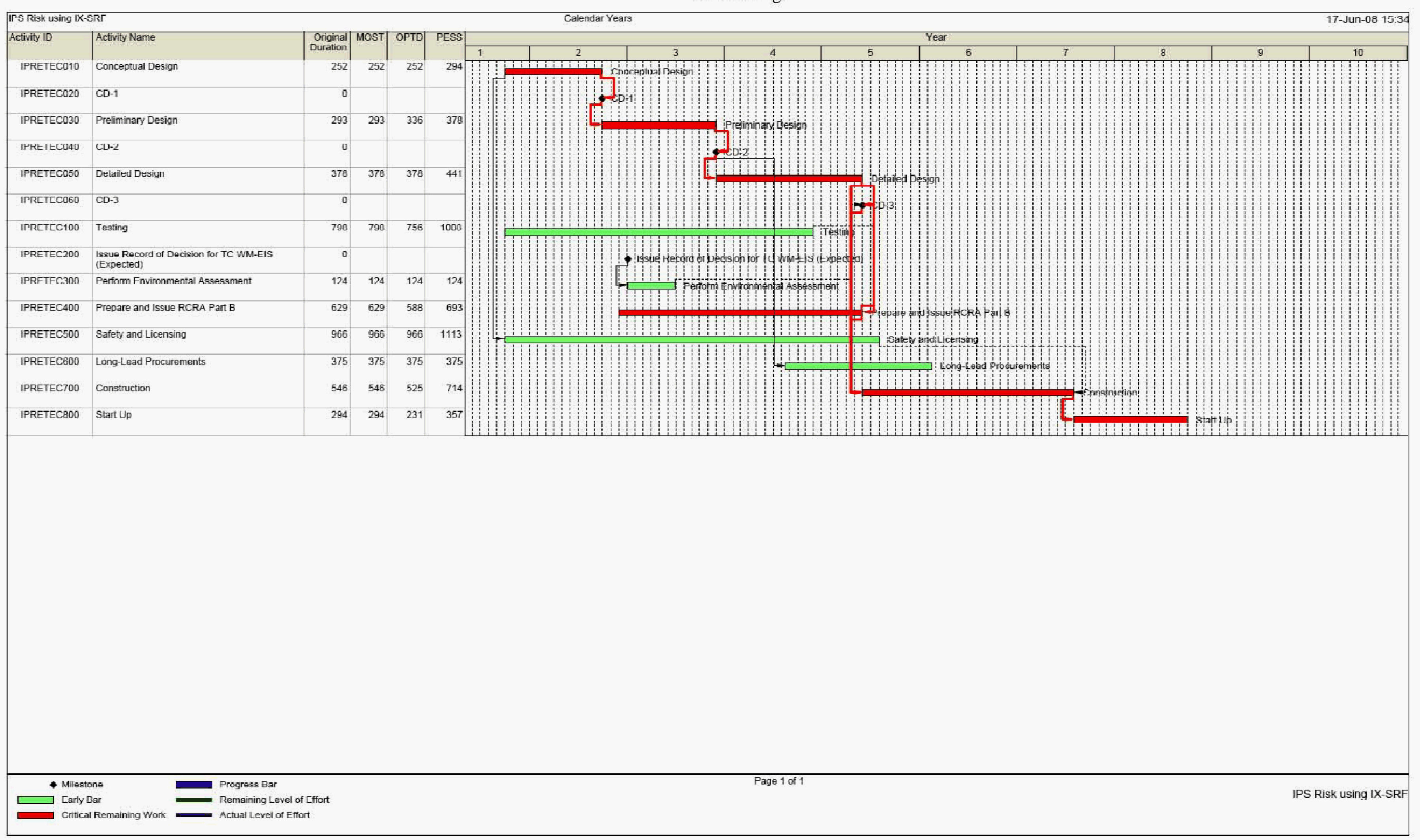

